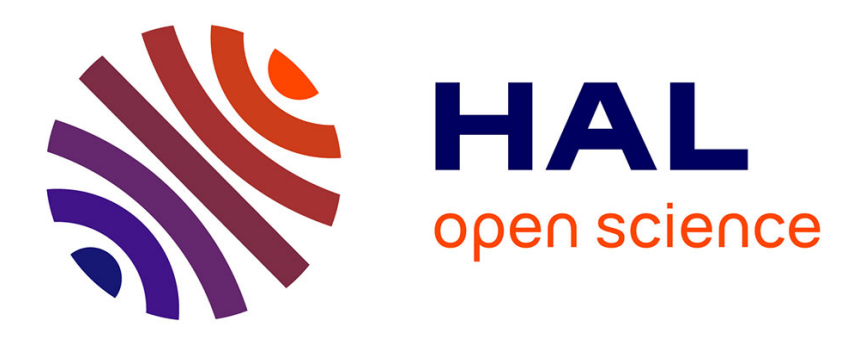

\title{
Dissection of the role of natural killer cells in atherosclerosis using selective genetic approaches \\ Wared Nour Eldine
}

\section{To cite this version:}

Wared Nour Eldine. Dissection of the role of natural killer cells in atherosclerosis using selective genetic approaches. Immunology. Université Sorbonne Paris Cité; Université Libanaise, 2017. English. NNT: 2017USPCB036 . tel-02117786

\section{HAL Id: tel-02117786 \\ https://theses.hal.science/tel-02117786}

Submitted on 2 May 2019

HAL is a multi-disciplinary open access archive for the deposit and dissemination of scientific research documents, whether they are published or not. The documents may come from teaching and research institutions in France or abroad, or from public or private research centers.
L'archive ouverte pluridisciplinaire HAL, est destinée au dépôt et à la diffusion de documents scientifiques de niveau recherche, publiés ou non, émanant des établissements d'enseignement et de recherche français ou étrangers, des laboratoires publics ou privés. 

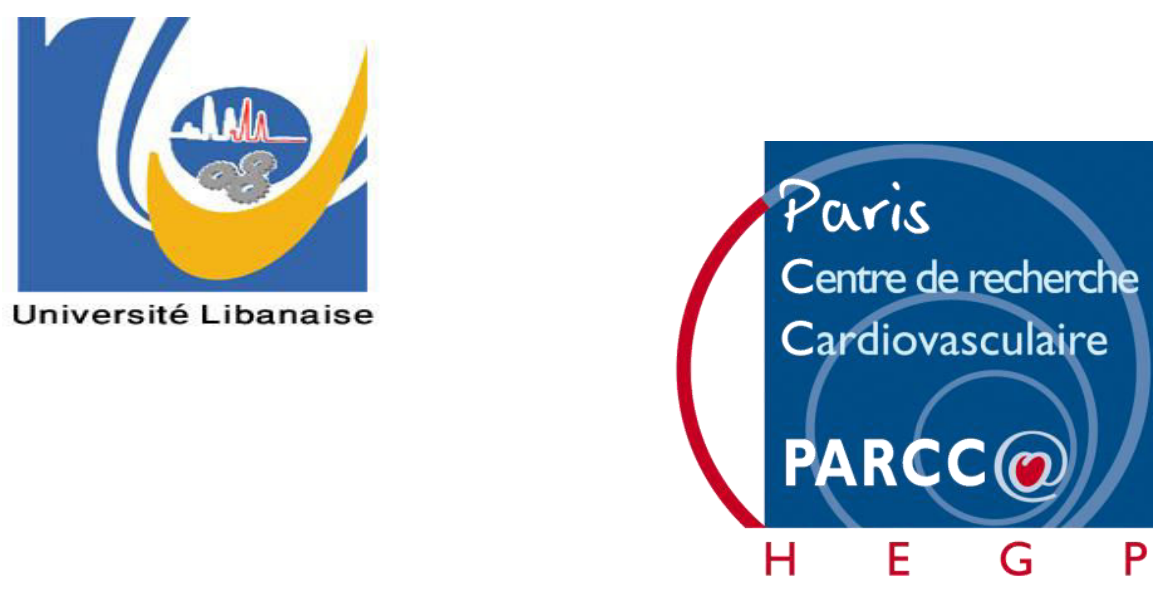

UNIVERSITÉ PARIS

Université Libanaise DESCARTES

Université Paris V - René Descartes

et

Université Libanaise

THÈSE DE SCIENCES

Pour l'obtention du titre de Docteur

École Doctorale Bio Sorbonne Paris Cité

Discipline : Biologie Cellulaire

\section{Dissection of the Role of Natural Killer Cells in Atherosclerosis Using Selective Genetic Approaches}

\section{Présentée et soutenue publiquement par}

Wared Nour ELdine

Le 06 Octobre 2017

\begin{tabular}{|l|l|l|}
\hline Auteur & \multicolumn{2}{|l|}{ Wared Nour Eldine } \\
\hline Composition du Jury & Pr. Jean-Francois Arnal & Rapporteur \\
\cline { 2 - 3 } & Pr. Aida Habib Abdul Karim & Rapporteur \\
\cline { 2 - 3 } & Pr. Sophie Ugolini & Examinateur \\
\cline { 2 - 3 } & Pr. Antonino Nicoletti & Examinateur \\
\hline Projet doctoral dirigé par & $\begin{array}{l}\text { Dr. Alain TEDGUl et Dr. Kazem } \\
\text { ZIBARA }\end{array}$ & Directeur de thèse \\
\hline
\end{tabular}




\section{Acknowledgments}

I would like first to convey deep thanks to the members of the Jury, Pr. Jean Francois Arnal and Aida Habib, Pr. Sophie Ugolini and Antonino Nicoletti for participating in the committee to evaluate my work. Thanks for your time, extreme patience, and intellectual contribution. I am honoured having each of you as a member in the committee.

To my Lebanese supervisor, Pr. Kazem Zibara, I offer my sincerest gratitude and appreciation. With you, I stepped to the world of scientific research. It is you, who paved for me the way, trusted, and encouraged me. With all meanings of thanks, I would thank you...

To Pr. Alain Tedgui, my unique supervisor, no words can describe my gratitude and affection, I am not fortunate but blessed to be your student, very much touched by your kindness and generosity before you scientific intelligence, thanks for your guidance throughout my PhD... Thanks for your spirit, which have inspired and will always inspire me...

A particular debt of gratitude is owed to Pr. Ziad Mallat for giving me the opportunity and welcoming me to be part of his team, where I have learned and grown both scientifically and personally... Thanks for sharing your exceptional scientific knowledge and your uttermost precious contributions whenever needed!

Much thanks goes to our Collaborators in Marseille, the group of Eric Vivier and Sophie Ugolini, for your expertise in NK cell world, for the valuable discussions we had, and for being always there when needed albeit the many kilometres from Paris....

I would like to distribute my thanks to the dream team "Equipe 5", for each one of you I offer a bundle of love and appreciation...

Our papy, Bruno, for all the aortas and hearts you've taken, Ludivine for your amazing organization and rules, Jeremie, a valuable guide, with whom I started my journey, Lynda and Yacine, my partners in this journey, as well as Marie and Andreas for the French conversations, helping me conquer my fear of mice :p It would have been impossible without your hand!

Yujao; the kindest and loveliest "Cicii" ever, Sarvenaz, Nada joe, Hajer, Anthony, Jennifer, Mona, Anna, Alice, Juliette, and Icia, Thanks all for your company...

My ambitious student, Malak, greetings for your good humour and all those moments you made me laugh...

Thanks for our CRs: Hafid who co-supervised me for the first year of my PhD, Soraya and Stephane, for monitoring and holding the Lab's motor... 
For Jose, the multitalented guy, open heart and hands for everyone in PARCC, I'm deeply impressed by your personality, Special Thanks to you and may your kindness and smile inspire the next generations to come...

My partners in the office, much Love and Thanks, Francine for your endless news, Isabelle for your kindness and valuable advices, and Laetitia for your expertise...

Miss Sihem, one friend for all, thanks for the early afternoon whispers, coffee breaks, and for the endless hindu series.....:p

To Sophie, the sweet Greek girl, for the love, soft heart, and warm hugs between the floors and the couloir.... My Greetings :D

To the friends of the third floor: Afef, Caterina, Hira, Anja, Louise, Ikram, Amel, Sylvian and Ludovic, Nirmine of fondation Carpentier, Dr. Nabila, who is "Nabila" as her name tells, Mengyao, and Siying of Team 3 ... My Greetings too...

For the Lebanese community in PARCC from the first to the third floor; Hasan, Nancy, Zainab, Somaya, Waed, Rami, Thanks for all the good moments we shared once!

For the animal house and its people, beginning from the head Elizabeth to all of you, Dominique, Nicolas, Emma, Corrina, Arex, Thanks for your kindness and taking good care of my mice!

For the administrative platform; Muriel, the two Martine, Philippe, Bruno and especially Veronique, Annette ... For the platform of Flowcytometry, Coralie then Camille, the platform of Histology, Micheline then Corrine... and the platform of Anatomopathology at HEGP, Pr. Patrick and the cute Megumi...and Finally, Madame Magazin "Stephanie”... Many Thanks...

For all the teams in PARCC, beginning with Team 1; Adele, Marwan, Julliette, Johanne, Min, Marion, and Xavier, Team 3, Irmine, Team 6, Ivana and Mathilde, Team 10, Thi, Marie, and Chehrezad, and Christian Stockman's team, Ewelina and Corrine, Thanks all for your indispensable cooperation and help...

Thanks for the PARCC and its people, who are undoubtedly all kind and nice. I believe that every one of you contributed to my success with a word or even a smile...

For my Lebanese Friends in Paris, with whom I wandered around and discovered Paris and the world, Lina, Ihsan, Mariam, and Racha, Precious Thanks...

For those who aren't mentioned here but have their place deep in my heart, Much Love! 
For my Family, my parents and lovely siblings, the reason behind every success and inspiration, for your infinite love and care... Love you all and to you I dedicate this work!

For the One and only One, who gave me these words to thank others, Thanks for everything I have, your messages, your blessings, your givings and gifts "And, as for the grants of your lord, then do narrate", It is but you, the origin, the roots, the beginning and the infinity of everything beautiful, In your name I begin and I close this speech and every stage in my life! 


\section{Table of Contents}

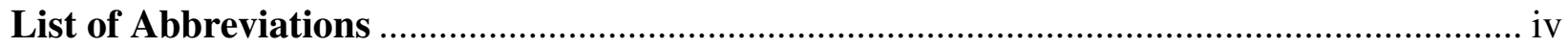

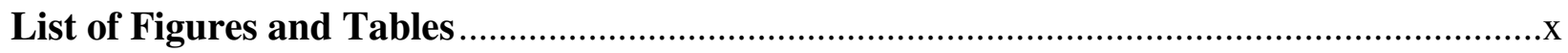

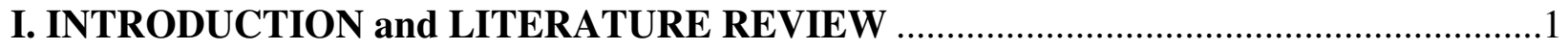

-Chapter 1-Atherosclerosis-A Cardiovascular Disease ....................................................2

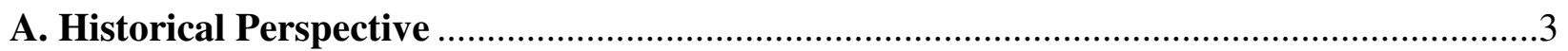

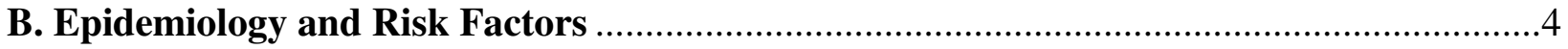

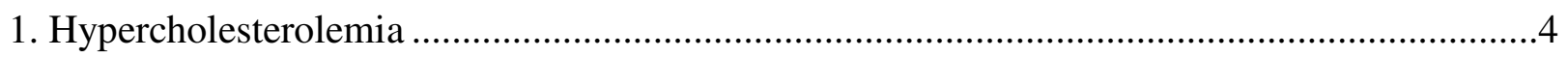

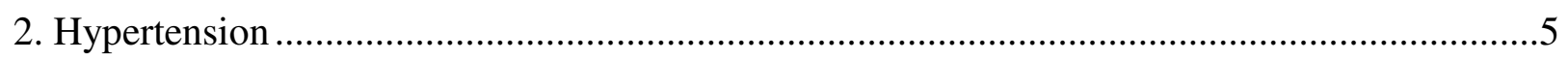

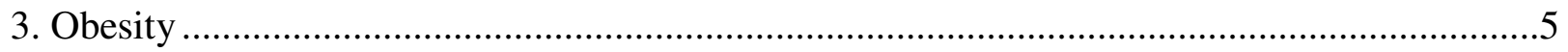

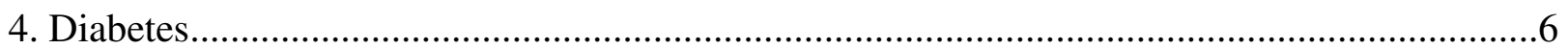

5. Autoimmune/Autoinflammatory diseases ................................................................6

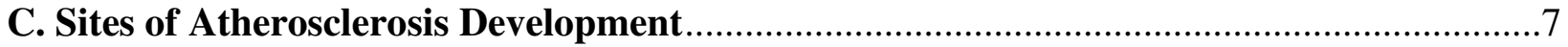

D. Classification of Atherosclerotic plaques .............................................................

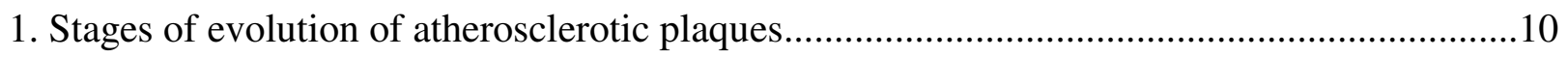

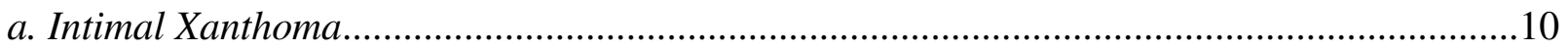

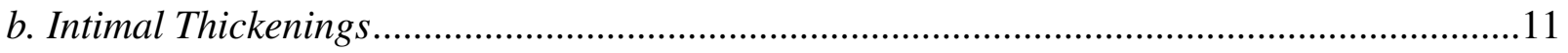

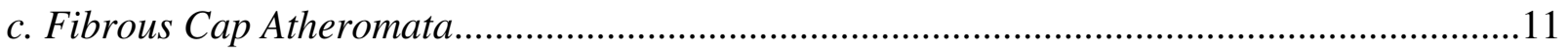

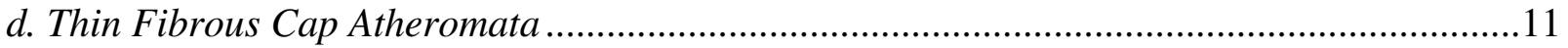

2. Lesions associated with clinical complications .......................................................11

a. Lesions associated with presence of thrombus ...............................................................11

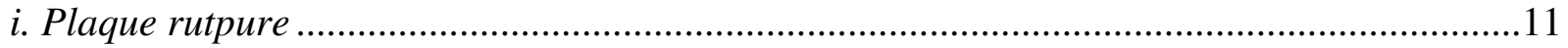

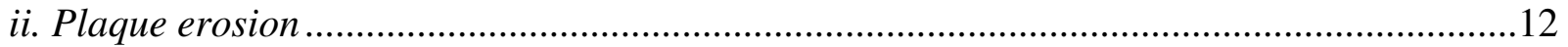

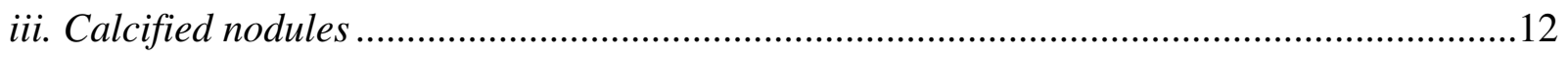

b. Lesions not associated with presence of thrombus ..............................................................12

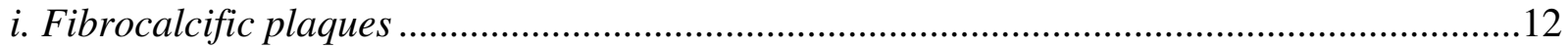

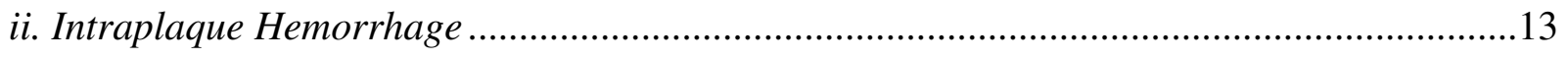

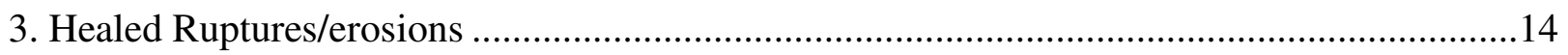

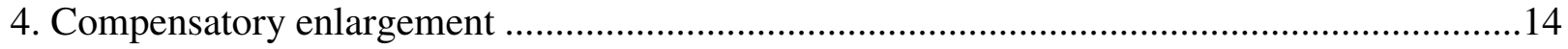




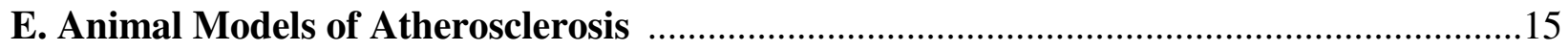

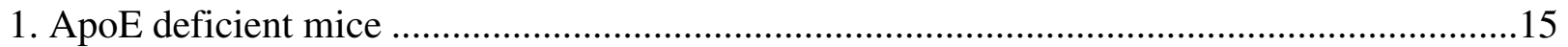

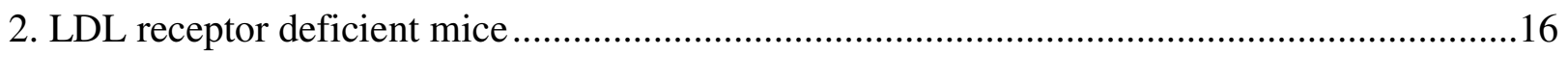

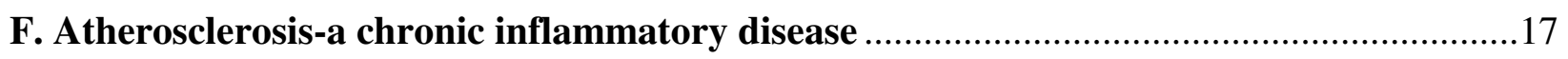

1. Changes in the vessel wall associated with development of lesions .......................................17

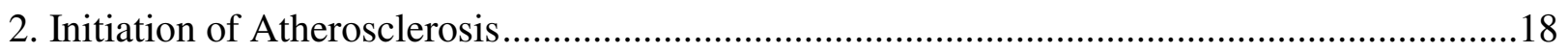

3. Endothelial cell activation and Leukocyte recruitment .........................................................19

4. Amplification of the inflammatory response and lesion progression ......................................20

5. Role of innate and adaptive immune cells in atherosclerosis ..................................................22

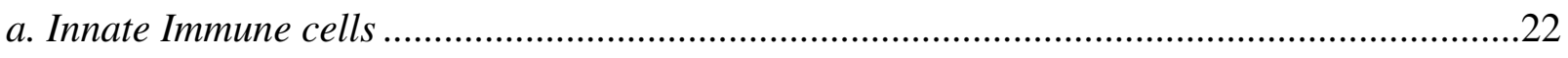

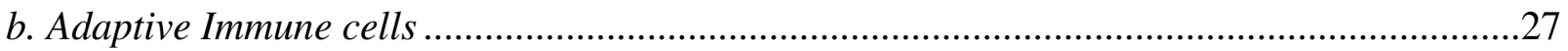

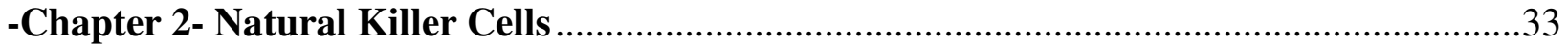

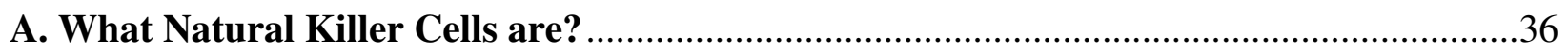

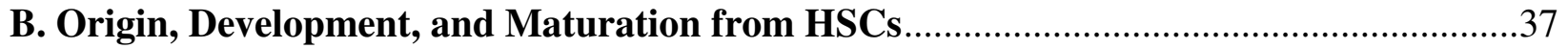

1. Generation of NK cell progenitors from HSCs....................................................................37

2. Developmental stages from NKP to mature NK cells ............................................................38

C. NK cell Trafficking and Tissue Distribution …………...................................................

D. NK cell Receptors and Ligands ...............................................................................

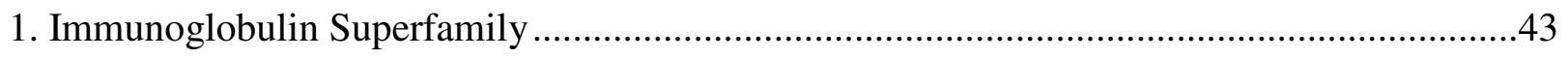

a. Killer immunoglobulin receptors (KIR) family ....................................................................4

b. Leukocyte Ig-like inhibitory receptors ……………………..................................................4

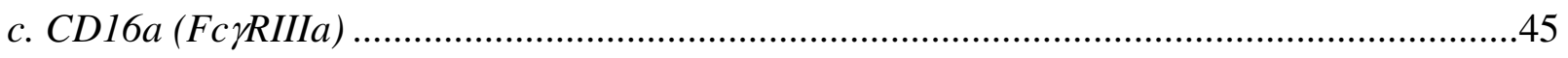

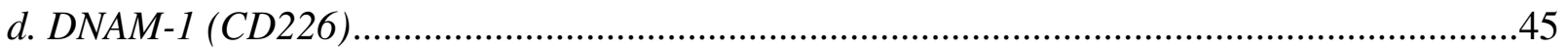

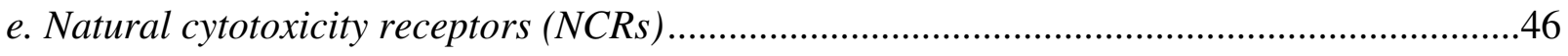

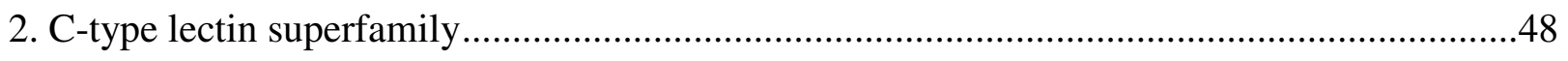

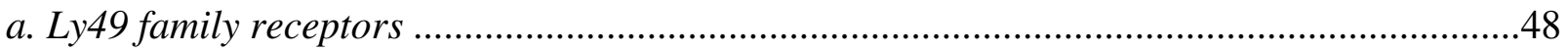

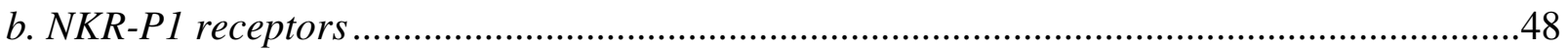

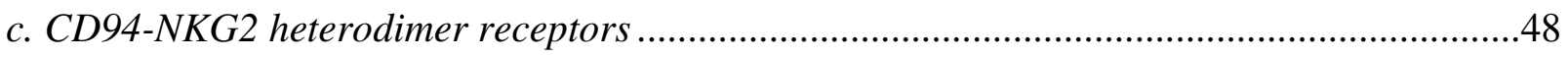

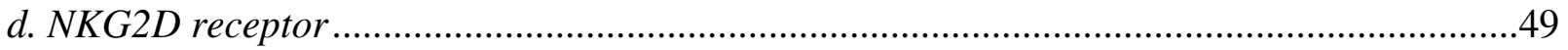

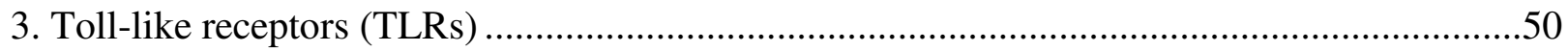

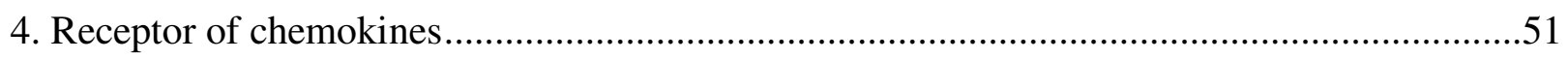




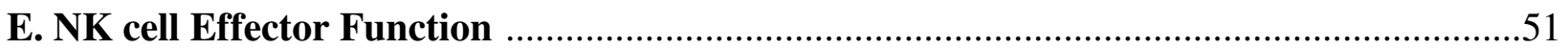

1. Granule exocytosis pathway …………………………....................................................

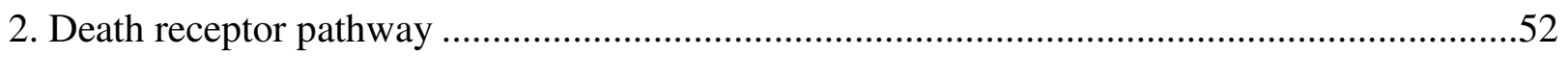

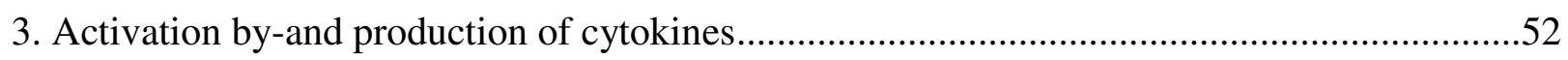

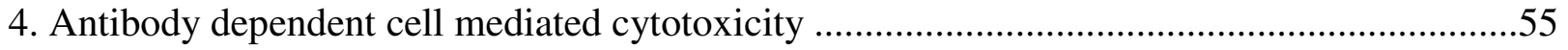

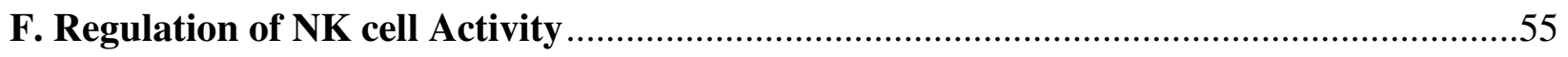

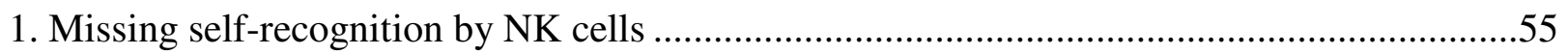

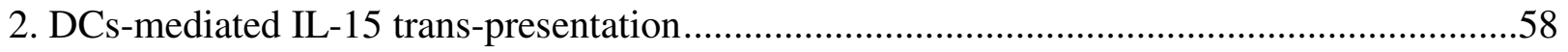

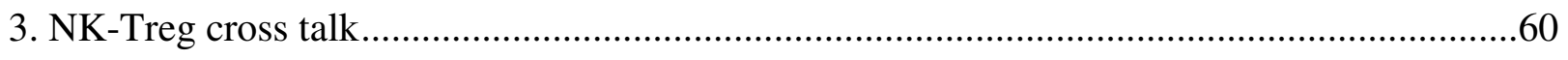

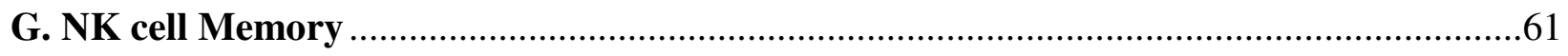

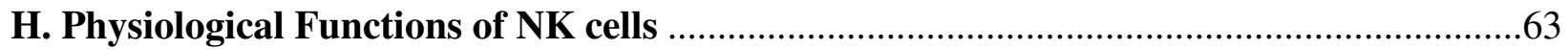

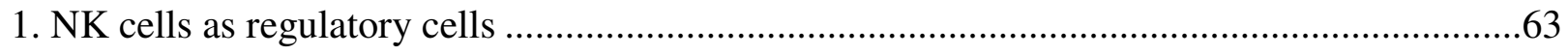

2. Anti-tumoral and anti-viral activities of NK cells ................................................................64

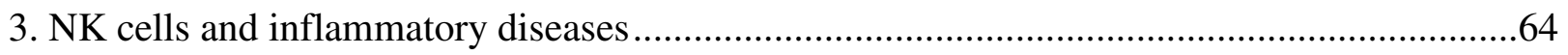

-Chapter 3- NK cells and Cardiovascular Diseases ………...................................................66

II. OBJECTIVES

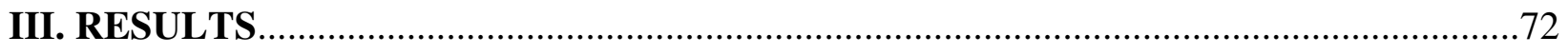

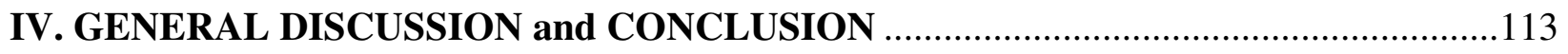

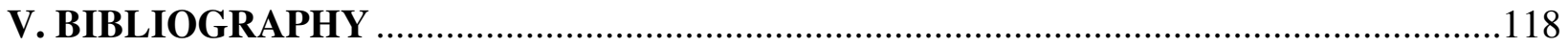




\section{List of Abbreviations:}

A

ADCC Antibody dependent cell mediated cytotoxicity

AGE Advanced glycation end

AHA American heart association

AIDS Acquired immune deficiency syndrome

AML Acute myeloid Leukemia

Ang II Angiotensin II

APC Antigen presenting cell

ApoE Apolipoprotein E

B

BAT3 Antigen-B-associated transcript 3

Bcl-2 B-cell lymphoma 2

BM Bone marrow

C

CAD Coronary artery disease

CBV Coxsackie virus B

CCL Chemokine ligand

CCR C-C chemokine receptor

$\mathrm{CD} \quad$ Cluster of differentiation

CLP Common lymphoid progenitor

CRP C-reactive protein

CSF Colony stimulating factor

CTL Cytotoxic T lymphocyte

CVD Cardiovascular disease

CXC C-X-C motif Chemokine 
D

DC Dendritic Cell

DNAM-1 DNAX accessory molecule-1

dsRNA Double-stranded RNA

$\mathbf{E}$

EAE Experimental autoimmune encephalomyelitis

EC Endothelial cell

eNOS endothelial Nitric oxide synthase

ER Endoplasmic reticulum

ERK Extracellular signal-regulated kinase

ERT EAT-2 related transducer

ESL1 E-selectin ligand 1

$\mathbf{F}$

FcR Fc receptor

G

GM-CSF Granulocyte-macrophage colony-stimulating factor

GPI Glycoprotein I

Grz Granzyme

H

HA Hemagglutinin

HCMV Human cytomegalovirus

HDL High-density lipoprotein

HLA-DR Human leukocyte antigen - antigen D related

HMG-CoA Hydroxymethyl glutaryl-coenzyme A

HSC Hematopoietic stem cell

HSP Heat-shock protein

HSPG Heparan sulfate proteoglycan 


\section{I}

ICAM-1 Intracellular adhesion molecule 1

IDL Intermediate-density lipoprotein

IDO Indoleamine 2,3-dioxygenase

IEL Intestinal epithelial lymphocyte

IgM Immunoglobulin M

IL Interleukin

ILT LIRIg-like transcript

iNKT Invariant natural killer T

iNOS inducible Nitric oxide synthase

IP-10 Inducible protein-10

IRF3 Interferon regulatory factor 3

IS Immunological synapse

ITAM Immunoreceptor tyrosine-based activating motif

ITIM Immunoreceptor tyrosine-based inhibitory motif

$\mathbf{J}$

Jak3 Janus kinase 3

JNK Janus kinase

$\mathbf{K}$

KIR Killer immunoglobulin receptors

$\mathbf{L}$

LAMP-1 Lysosome associated membrane protein-1

LDL Low-density lipoproteins

Ldlr Low density lipoprotein recptor

LFA-1 Lymphocyte function-associated antigen-1

LIR Leukocyte immunoglobulin inhibitory receptor

LN Lymphe node

LOX-1 Lectin-like oxidized low density lipoprotein receptor 1 
LPS Lipopolysaccharide

LTi Lymphoid tissue inducer

Ly6c Lymphocyte antigen 6 complex

Ly49 Lectin like receptors

M

MAPK Mitogen activated protein kinase

MCP-1 Monocyte chemoattractant protein-1

MDA5 Melanoma differentiation-associated protein-5

MHC Major Histocompatibility complex

MI Myocardial infarction

MIC Class I chain-related molecules

MMP Matrix metalloproteinase

MULT-1 Murine UL 16-binding protein like transcript 1

$\mathbf{N}$

NADPH Nicotinamide adenine dinucleotide phosphate

NCR Natural cytotoxicity receptor

NF-kB Nuclear factor $k B$

NK Natural killer

NKC NK gene complex

NO Nitric oxide

$\mathbf{O}$

OP Osteoporotic

oxLDL Oxidized low density lipoprotein

PAI-I Plasminogen activator inhibitor-1

PAMP Pathogen associated molecular pattern

PCNA Proliferating cell nuclear antigen

PD-1 Programmed cell death protein 1

pDC Plasmacytoid Dendritic cell 
PDGF Platelet derived-growth factor

PGE 2 Prostaglandin E2

PKC Protein kinase $\mathrm{C}$

PSGL1 P-selectin glycoprotein ligand 1

$\mathbf{R}$

RA Rheumatoid arthritis

RAET-1 Retinoic acid early transcript-1

RAG Recombination-activating gene

RAGE AGE receptor

ROS Reactive oxygen species

$\mathbf{S}$

SAP Serum amyloid P component

SHP1 SRC homology phosphatase 1

SLE Systemic lupus erythematosus

SMC Smooth muscle cell

SR Scavenger receptor

SREC1 Scavenger receptor class F member 1

STAT5a Signal transducer and activator of transcription 5A

$\mathbf{T}$

TCR T cell receptor

TGF- $\beta \quad$ Transforming growth factor- $\beta$

TH1 Thelper type 1

TICAM-1 Toll-IL-1 receptor domain containing adaptor molecule-1

TIR Toll-interleukin 1 receptor

TLR Toll like Receptors

TNF Tumor necrosis factor

TRAIL TNF-related apoptosis-inducing ligand 
ULBP UL-16 binding protein

V

VCAM-1 Vascular cell adhesion molecule 1

VEGF Vascular endothelial growth factor

VLA-4 Very late antigen-4

VLDL Very low density lipoprotein

VSMC Vascular smooth muscle cell

W

WHO World health organization

WT Wild type

$\mathbf{Z}$

ZAP70 Zeta-chain-associated protein kinase 70 


\section{List of Figures and Tables}

Figure 1: Sites of atherosclerotic lesion formation in humans and mouse ...............................8

Figure 2: Simplified scheme of the classification of lesions modified by Virmani after AHA Recommendation.

Figure 3: Main lesion types of atherosclerosis and proposed sequence of their development ....13

Figure 4: Human coronary artery with compensatory enlargement …...................................14

Figure 5: Stages in the development of atherosclerotic lesions ...........................................21

Figure 6: Immune components of the atherosclerotic plaque .............................................24

Figure 7: Role of different CD4 T cell subsets ..................................................................28

Figure 8: Phenotypic markers of developing NK cells in the mouse .......................................39

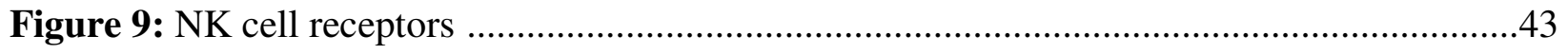

Figure 10: Structure of Natural Killer cytotoxicity receptors ..............................................46

Figure 11: Signaling pathways leading to NK cell activation .............................................54

Figure 12: Balance between inhibitory and activating signals governs NK cell function............55

Figure 13: Models proposed for NK cell education. ...........................................................57

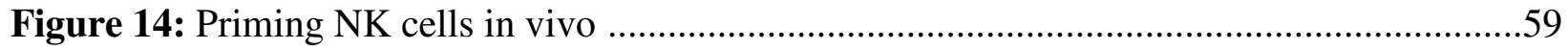

Figure 15: IL-2 dependent Treg -NK cell cross talk.........................................................60

Figure 16: Three paths towards becoming a memory NK cell ..............................................62

Figure 17: Proposed mechanism of NK-cell enhanced atherogenecity in an inflammatory context

\section{$\underline{\text { List of Tables }}$}

Table 1: Histological classification of the stages of atherosclerosis proposed by AHA ..............9

Table 2: Role of different innate and adaptive immune cells in atherosclerosis development.....34

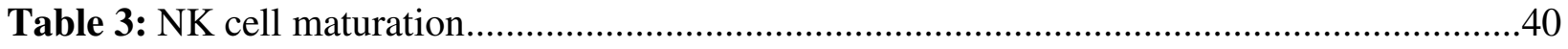




\section{INTRODUCTION}

E

LITERATURE REVIEW 


\section{-CHAPTER 1 - \\ Atheroscterosis-A \\ Cardiovascular \\ Disease}




\section{A. Historical perspective}

Even though atherosclerosis is reaching epidemic proportions nowadays, it is not in anyway a disease specific to modern days. It is rather an ancient disease with a fascinating history, firstly characterized in medical works of ancient Egyptians, Greeks, and Romans [1], [2].

Nowadays, atherosclerosis is recognized as a chronic inflammatory disease of large and medium sized arteries, characterized by the accumulation of low-density lipoproteins (LDL) and the recruitment of circulating monocytes and $\mathrm{T}$ cells in the intima, leading to the formation of macrophage derived foam-cells, as well as the proliferation of smooth muscle cells (SMCs), and accumulation of collagen and proteoglycans in the fibrous cap [3], [4].

However, its recognition and characterization progressed over centuries. In the latter half of last millennium (1575), the anatomist Fallopius wrote of "degeneration of arteries into bone". Later on, in 1740, the German physician Krell described for the first time "calcium concretions" in the arterial wall of aged subjects, which he called "bony plates" [5].

The term "atheroma" was first introduced in 1755 by Albrecht von Haller to designate the plaque deposited on the innermost layer of systemic artery walls. Then in 1904, Felix Marchand suggested the word "atherosclerosis" should be better used to refer to the degenerative process of the intimal layer of arteries, which derives from athera, meaning "porridge" or "gruel" in ancient Greek, and "skleros" signifying "hard" in Greek.

Later, in 1815, Hodgson proposed that inflammation is the underlying cause of atheromatous arteries. However, the first mechanistic assessment of atherosclerosis was initiated in 1908 by the Russian scientist Alexander Ignatowski who demonstrated that rabbits fed a diet of milk and egg yolk develop atherosclerosis. Subsequently, in 1913, Anitschkov and Chalatov reproduced experimental atherosclerosis by adding pure cholesterol ("cholesterin" as it called at that time) to rabbit food [6]. Since then, the lipid theory of atherosclerosis was established and predominated for most of the 20th century [1].

Soon after, this concept was replaced by the "response to injury hypothesis" which dates back to the pioneering work of Virchow and Carl von Rokitansky who discovered inflammatory cells in the developing lesions. This theory was later developed by Russel Ross (1976), who proposed that endothelial injury leads to platelet adhesion and smooth muscle cell proliferation [7].

At that time, it was believed that SMC proliferation, drived by platelet derived-growth factor (PDGF) secreted from platelets, is the principal factor occluding the artery. However, it was later established that SMC proliferation is rather modest and it seems to be beneficial by 
contributing to plaque stabilization. Later, when Poole and Florey observed monocytes adherence and migration to the endothelium after feeding cholesterol to rabbits, Ross revisited his "response to injury theory" and published in the New England Journal of Medicine a remarkable review entitled: "Atherosclerosis: a chronic inflammatory disease" [8].

The precise identification of cell components of human atherosclerotic plaques using modern immunohistochemical techniques by Göran Hansson and his colleagues was instrumental in the change of opinion regarding the role of inflammation and immunity, rather than SMC proliferation, in the pathogenesis of atherosclerosis. Histologically, the lipid laden foam cells originate from macrophages and large amount of T cells, about $20 \%$, surround the plaque and are present in the fibrous cap, pointing to a role of immunity in atherosclerosis [9],[1].

It is now widely accepted that atherosclerosis is a chronic inflammatory disease of the artery wall where immune reposnes are involved at all stages of the disease.

\section{B. Epidemiology and Risk Factors}

According to the World Health Organization (WHO), cardiovascular diseases (CVD) are the leading cause of mortality worldwide accounting for 17.7 million victims in 2015 (WHO report 2017), which represents $30 \%$ of all global deaths, mainly from ischemic heart disease and stroke. If the current trend continues, this number is predicted to increase to 26 million deaths in 2030 [10].

Although CVD and related chronic diseases were considered to be diseases of industrialized countries, WHO reported that $80 \%$ of the 17.7 million cardiovascular deaths in 2015 took place in low and middle-income countries (WHO report 2017).

The likelihood of occurrence of CVD increases in the presence of multiple risk factors for atherosclerosis. These are modifiable or non-modifiable. The non-modifiable are: age, gender, and family history. The modifiable risk factors are hypercholesterolemia, hypertension, smoking, diabetes mellitus, obesity, and sedentary lifestyle [11].

\section{Hypercholesterolemia}

Among the many cardiovascular risk factors, elevated plasma cholesterol level is probably unique in being sufficient for the development of atherosclerosis, even in the absence of other known risk factors. If all adults had plasma cholesterol levels $<150 \mathrm{mg} / \mathrm{dl}$, symptomatic disease would be rare [12].

Low-density lipoprotein (LDL) (which constitutes $70 \%$ of total serum cholesterol) 
accumulating in the intima can be oxidized and initiate inflammation by increasing the expression of adhesion molecules by the endothelium, and chemokines and cytokines by macrophages. The five single-gene disorders that result in elevated LDL levels are associated with premature coronary atherosclerosis [13].

In addition, lipid-lowering agents, such as statins (HMG-CoA reductase-inhibitors) significantly reduce cardiovascular mortality, both in primary or secondary prevention [14]. Recent clinical trials have shown that reduction in the rate of coronary events is directly related to the magnitude of reduction in LDL cholesterol levels [13].

\section{Hypertension}

Epidemiological studies clearly pointed out that arterial hypertension is a major risk factor for CVD, especially stroke. Elevated blood pressue levels have been highly predictive of atherosclerosis associated-cardiovascular events. In human subjects, carotid artery intimamedia thickness is highly correlated with blood pressure levels, and accurately reflects $\mathrm{CV}$ risk. This is supported by experimental evidence demonstrating that hypertension increases the rate of atherosclerotic plaque development in hypercholesterolemic rabbits, monkeys, and mice.

Hypertension presents in several forms, some linked to activation of the renin-angiiotensin system and elevated circulating Angiotensin II (Ang II), and some with normal Ang II levels. A study by Mazzoli et al reported that hypercholesterolemic mice (Apoe ${ }^{-/-}$) with high circulating or normal Ang II levels exhibited similar atherosclerosis plaque size, but those of hypertensive animals had signs of instability, as well as enhanced systemic inflammation [15].

Several mechanisms can account for hypertension-induced atherosclerosis. Firstly, the pressure-induced stretch of the wall increases endothelial permeability to low-density lipoprotein and their subsequent retention ton the intima. Also, hypertension aggravates endothelial dysfunction, enhances monocyte adhesion, and activates the transcription of inflammatory genes [16]. All these factors are central to the development of atherosclerosis and will be discussed through the chapter.

\section{Obesity}

Several clinical and subclinical abnormalities are found in obese patients, such as insulin resistance, atherogenic dyslipidemia, prothrombotic and proinflammatory state, all of which contribute to the development of atherosclerotic plaques and their complications [17].

Obese subjects carry a proinflammatory state characterized by elevated levels of C-reactive 
protein (CRP), which reflects high cytokine levels secreted by excess adipose tissue. This may render stable atherosclerotic plaques vulnerable to rupture [18], [19]. In addition, increased production of plasminogen activator inhibitor-1 (PAI-1) by adipose tissue favors a prothrombotic state [20]. Circulating Tumor necrosis factor- $\alpha$ (TNF- $\alpha$ ) is markedly increased in obese patients [21]. Moreover, leptin, synthesized by adipose tissue, plays a role in obesity-induced endothelial dysfunction. It increases oxida0tive stress in endothelial cells, promotes vascular cell calcification, and smooth muscle cell proliferation and migration [22].

\section{Diabetes}

Diabetes is defined as fasting blood glucose of $126 \mathrm{mg} / \mathrm{dl}$ or greater. Persons with type 1 and type 2 diabetes are at increased risk for coronary artery disease (CAD).

For example, in a Finnish population-based study, the seven-year incidence of myocardial infarction among 1373 non-diabetic subjects with and without pre-myocardial infarction at baseline was $18.8 \%$ and $3.5 \%$ respectively. However, in 1059 persons with type 2 diabetes, the incidence rates of myocardial infarction increased to $45 \%$ and $20 \%$, respectively [13].

Hyperglycemia induces the formation of reactive oxygen species (ROS) and advanced glycation end products (AGE), which plays a major role in the pathogenesis of diabetes, mainly through endothelial dysfucntion. AGEs exert their pathogenic effects by engaging binding sites/receptors. They have been shown to activate macrophages in an NF-kBdependent fashion, leading to induction of proinflammatory cytokines, such as IL-1 $\beta$ and TNF- $\alpha$. In addition, their binding to the endothelial receptor for AGE (RAGE) results in depletion of cellular anti-oxidant defense mechanisms (glutathione, vitamin $\mathrm{C}$ ) and the generation of ROS. Increased cellular oxidative stress induces the endothelium to express the procoagulant tissue factor and adhesion molecules such as E-selectin, intracellular adhesion molecule 1 (ICAM-1), and vascular cell adhesion molecule 1 (VCAM-1).

In addition, hyperglycemia increases the matrix metallopeptidase 9 (MMP-9) activity, partly due to increased transcription of MMP-9 through redox-sensitive mechanisms. MMPs are involved in monocyte invasion, the vascular smooth muscle cell (VSMC) migration and collagen degradation; its dysregulation is a critical factor in development of vascular lesionsand plaque instabilty [23].

\section{Autoimmune/Autoinflammatory diseases}

Chronic infections [24] and autoimmune diseases, specifically rheumatoid arthritis (RA) [25] and systemic lupus erythematosus (SLE) [26], also represent a risk factor for atherosclerosis. 
Patients with autoimmune diseases, such as SLE or RA, are at particularly high risk of CVD. These patients benefit from treatment with antibodies directed against the B-cell receptor CD20.

The chronic systemic inflammation that occurs in RA can contribute to endothelial dysfunction and oxidative stress to promote atherosclerosis [27]. In patients with RA, antiTNF- $\alpha$ therapy reduced inflammation, thrombotic risk, and the incidence of cardiovascular events.

Accelerated atherosclerosis is a major source of morbidity in SLE. CD4 ${ }^{+}$T cells from SLEsusceptible mice transferred into Low density lipoprotein receptor $\left(\mathrm{Ldll}^{-/}\right)$deficient mice increased atherosclerosis [28].

\section{Sites of Atherosclerosis development}

Atherosclerosis localizes to reproducible sites of the vascular tree. Lesions are found primarily in large and medium sized muscular arteries [29].

Regions of arterial tree with laminar shear stress are resistant to the development of atherosclerosis due to orderly blood flow. However, branch points of arteries, which experience low shear stress, turbulence and oscillating flow, are predisposed to lesion formation [30]. These areas of arterial branches, bifurcations, and curvatures may be located in the coronary, cerebral, carotid, femoral arteries, and in the aorta [31], [32] (Figure 1A).

Before development of atherosclerosis, the predilection sites are characterized by changes in endothelial turnover and gene expression, [33] presence of subendothelial dendritic cells and in humans, by the presence of adaptive intimal thickening [34].

Fluid mechanical forces can directly influence endothelial cell structure and function [35]. Endothelial cells align with in the direction of laminar flow. In areas of disturbed flow, this alignment is abolished.

Endothelial cells express genes, which are differentially regulated by blood flow rate. These include cell surface adhesion molecules (VCAM-1), intracellular adhesion molecules (ICAM-1), pro-oxidant enzymes (lipoxygenases, NADPH oxidases) and antioxidant enzymes (NO synthase, superoxide dismutase) genes [36]. Oscillatory shear stress substantially upregulates VCAM-1, ICAM-1, and E-selectin in cultured human endothelial cells [37].

Nitric oxide (NO), the potent vasodilator with anti-inflammatory activities, is the end product of conversion of L-arginine to L-citrulline by the endothelial nitric oxide synthase (eNOS). eNOS is induced in a dose-dependent manner by laminar shear stress and downregulated by oscillatory shear stress [38]. One of the early responses to hypercholesterolemia is the 
attenuation of endothelium dependent production of NO. In addition to decreased vasodilation, deficiency in $\mathrm{NO}$ enhances SMC proliferation and platelet aggregation and adhesion [39], [40].
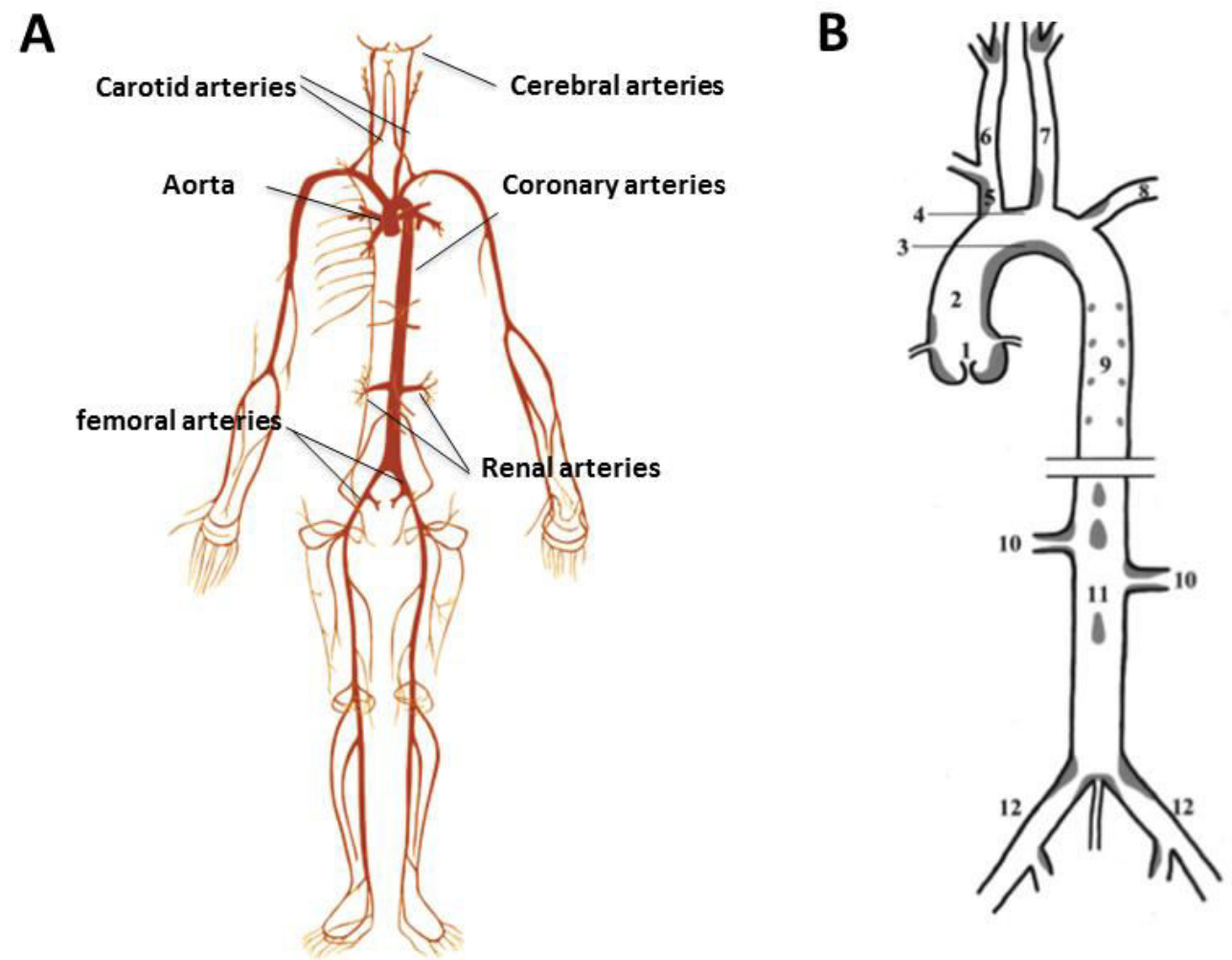

Figure 1: Sites of atherosclerotic lesion formation in humans and mouse (A) Shcematic shows the common sites of atherosclerosis formation in human (B) Schematic showing the major arterial vasculature distribution of atherosclerosis (gray shading) in $L d l r^{-/}$mice fed a high atherogenic diet. 1: indicates aortic sinus; 2: ascending aorta; 3: lesser curvature of aortic arch; 4: greater curvature of aortic arch; 5: innominate artery; 6: right common carotid artery; 7 : left common carotid artery; 8: left subclavian artery; 9: thoracic aorta; 10: renal artery; 11: abdominal aorta; 12: iliac artery. (adapted from Vanderlaan et al, ATVB-2004 and De Bakey, 1985)

Accumulating evidence shows that blood flow affects whether a plaque progresses into a vulnerable or stable plaque [29].

Similarly in mice, the development of atherosclerosis occurs at reproducible sites predetermined by the forces experienced by the endothelium. This vascular distribution is depicted in Figure 1B. These sites are precisely where turbulent, pulsatile, and non-laminar flow forces predominate [36]. 


\section{Classification of Atherosclerotic Plaques}

The pathogenesis of atherosclerotic lesions has been inferred from microscopic analysis of arteries in different age groups.

The classification of atherosclerotic lesions was initially proposed by Stary and his colleagues (1995), then adopted by the American heart association (AHA) based on the histological composition and structure of the plaque. This classification comprises seven stages of chronological evolution and increasing gravity [41], [42] (Table 1).

\begin{tabular}{|l|l|l|}
\hline Type & Term proposed & Description \\
\hline I. & Isolated macrophage foam cells & $\begin{array}{l}\text { Isolated macrophage foam cells in the } \\
\text { intima, absence of extracellular lipids. }\end{array}$ \\
\hline II. & Fatty streak & $\begin{array}{l}\text { Multiple layers of macrophage foam cells } \\
\text { and lipid laden smooth muscle cells }\end{array}$ \\
\hline III. & Pre-atheroma & $\begin{array}{l}\text { Scattered collections of extracellular lipid } \\
\text { droplets }\end{array}$ \\
\hline IV. & Atheroma & $\begin{array}{l}\text { Extracellular lipid accumulation referred to } \\
\text { as lipid core }\end{array}$ \\
\hline V. & Atherosclerotic plaque & $\begin{array}{l}\text { Lipid core and thick layers of fibrous tissue } \\
\text { mainly collagen }\end{array}$ \\
\hline VI. & Complicated atherosclerotic plaque & $\begin{array}{l}\text { Surface defect (Fissure), hematoma, } \\
\text { hemorrhage, thrombus }\end{array}$ \\
\hline VII. & Fibrotic plaque & $\begin{array}{l}\text { Massive intimal thickening by collagenous } \\
\text { sclerosis, extracellular lipids almost absent } \\
\text { or negligible }\end{array}$ \\
\hline
\end{tabular}

Table 1: Histological classification of the stages of atherosclerosis proposed by the AHA, (adapted from stary ey al, Circulation, 1995)

Later in 2000, Renu Virmani and Stephen Schwartz introduced an alternative and simpler classification, which emphasizes the link between lesion morphology and clinical disease. Lesions types recognized in this classification are displayed in Figure 2 [43]. 


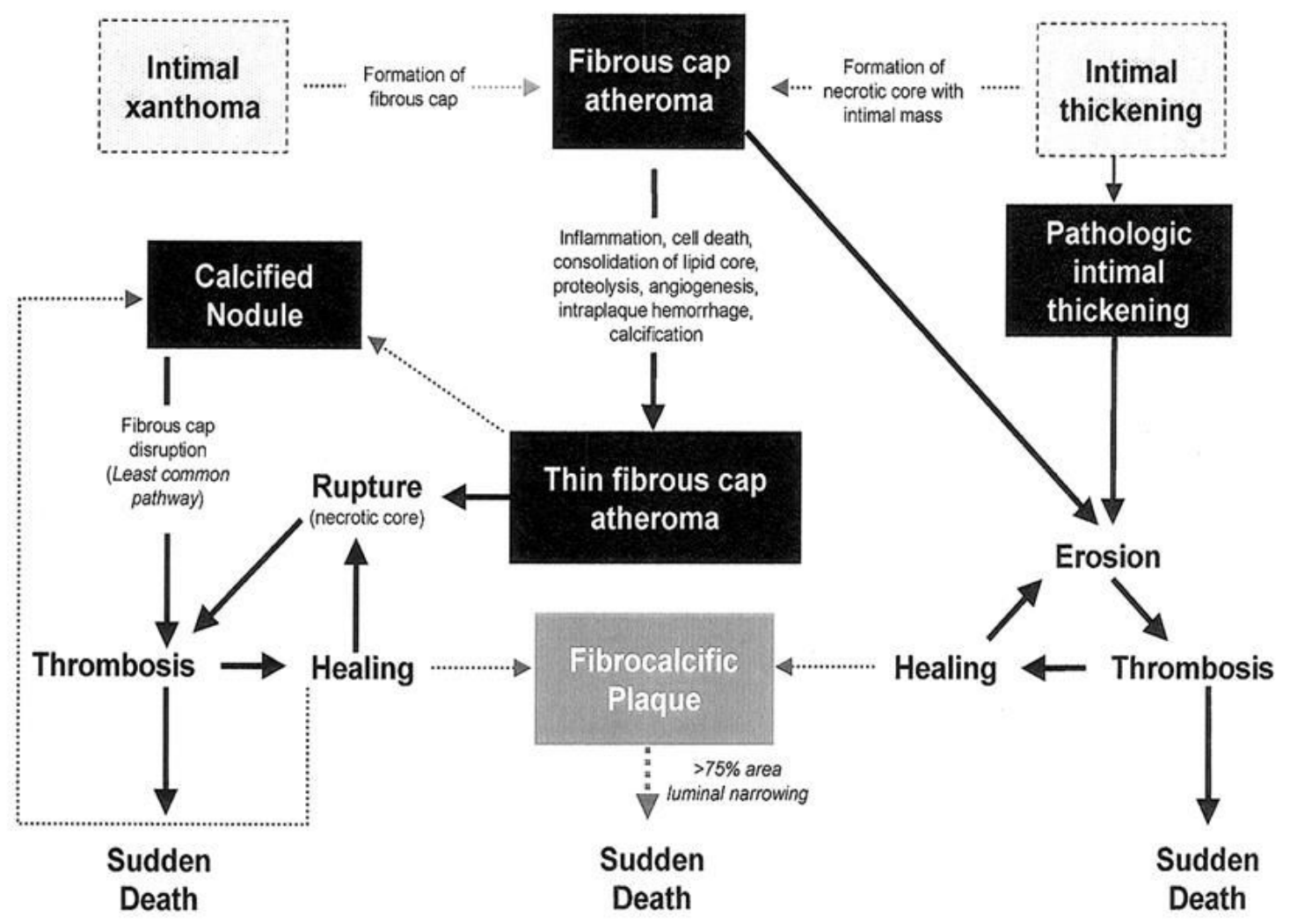

Figure 2: Simplified scheme of the classification of lesions modified by Virmani after AHA recommendation, The boxed areas represent the seven types of lesions. Dashed lines were used for two boxes because there is controversy about their roles in the initial phase of lesion formation and both lesions can exist without progression to a fibrous cap atheroma. The processes leading to lesion progression are listed between categories. Lines depict current concepts of how one category may progress to another, (adapted from Virmani et al, 2000, ATVB)

\section{Stages of evolution of atherosclerotic plaques}

The new scheme by Virmani et al [43] defines seven categories considering the pathophysiological progression, including accumulation of lipids, formation of cap and necrotic core, remodeling of the fibrous plaque and thrombosis. The main lesions of atherosclerosis and the proposed cellular and morphological sequential changes are represented in Figure 3 [44].

\section{a. Intimal Xanthoma}

This term was proposed instead of type I lesion, "fatty streak" or initial lesion in the AHA's scheme. Xanthoma is a general pathological term that describes focal accumulations of lipid laden-macrophages. In humans, intimal xanthomas present in children, probably reflecting risk factors of the mother, but appear to regress with age since they are rarely found in 
elderly [45]. Xanthomas are harmless and fully reversible if the stimuli that caused their formation dissipate [44].

\section{b. Intimal Thickenings}

Although the authors did not exclude the fact that some human lesions may begin as intimal xanthomata, they suggested that most adult human lesions originate as preexisting intimal masses. Indeed, the distribution of these intimal thickenings observed in children is correlated with the usual distribution of lesions of the adult [46].

\section{c. Fibrous Cap Atheromata}

It is defined as a distinct layer of connective tissue completely covering the lipid core. It consists purely of SMCs in a collagenous proteoglycan matrix, with varying degrees of infiltration by macrophages and lymphocytes. Thus, the fibrous cap atheroma may have thick or thin cap overlying a lipid rich core.

\section{d. Thin fibrous Cap Atheromata}

The aim behind adding this type of lesion, which was not recognized by the AHA classification, is that lesions with thin fibrous caps are most likely prone to rupture. Thin fibrous cap is defined as one which is $<65 \mu \mathrm{m}$ thick. This definition is after morphometric examination of a series of 41 ruptured plaques in which $95 \%$ of the caps measured $<64 \mu \mathrm{m}$ thick. Its content is distinguished from earlier fibrous cap lesions by loss of SMCs, extracellular matrix, and inflammatory infiltrate [47]. However, the thickness of the cap is not sufficient to allow prediction of plaque rupture. The latter necessitates other relevant features such as the extent of inflammation in the cap, fissuring, calcification, and intraplaque hemorrhage.

\section{Lesions associated with clinical complications}

Literature review revealed that plaque rupture is responsible for $76 \%$ of all heart attacks caused by coronary thrombosis worldwide. The remaining $24 \%$ are caused by plaque erosion and other less well defined mechanisms [12].

Many factors determine whether a thrombus occurs or not. Autopsy studies show that vulnerability is a function of increased number of macrophages, increased expression of tissue factor, reduced number of SMCs, large lipid core and thin plaque cap [48].

\section{a. Lesions associated with presence of thrombus}

\section{i. Plaque rupture}


In plaque rupture, a structural defect -gap- in the fibrous cap exposes the contents of necrotic core to circulating blood immediately causing more or less atherothrombotic process. Plaque rupture occurs where the cap is the thinnest and most infiltrated by foam cells [44].

Ruptured plaques are characterized by a large necrotic core and disrupted fibrous cap infiltrated by macrophages and lymphocytes, with a sparse SMC content. Ruptures are observed in $60 \%$ of individuals dying suddenly with luminal thrombi and are the most frequent cause of death in young men ( $<50$ years) and old women ( $>50$ years) [49].

\section{ii. Plaque erosion}

Virmani uncovered another mechanism of coronary thrombosis occurring in unruptured noninflammatory plaques, described as plaque erosion [1]. Eroded plaques differ from ruptured plaques in that they have a base rich in proteoglycans and smooth muscle cells.

Plaque erosion accounts for $20 \%$ of all sudden deaths or $40 \%$ of coronary thrombi in patients dying suddenly from coronary artery atherosclerosis. It affects mainly young individuals and women before menopause and is associated with smoking [50], [51].

They are associated with less luminal narrowing and less calcification, and they are less likely to have foci of macrophages and $\mathrm{T}$ cells compared with ruptured plaque. Recently studies provided experimental evidence that endothelial apoptosis might be a major determinant of plaque erosion [52].

\section{iii. Calcified nodule}

The least frequent lesion of thrombosis is referred to as calcified nodule. The luminal region of the plaque consists of breaks in the calcified plates, bone formation, and interspersed fibrin with a disruptive surface fibrous cap and an overlying thrombus. These lesions are found in midright coronary artery and might be related to the frequent occurrence of plaque hemorrhage [43].

\section{b. Lesions not associated with presence of thrombus}

\section{i. Fibrocalcific plaques}

These plaques are characterized by thick, fibrous caps overlying extensive accumulations of calcium in the intima close to the media. They are referred to as fibrocalcific due to the small lipid-laden necrotic core, if present. One hypothesis is that these plaques result from healing of an atheromatous plaque following incomplete rupture or erosion [53]. 


\section{ii. Intraplaque hemorrhage}

The pathogenesis of intraplaque hemorrhage is controversial and has been discussed for years. Constantinides originally suggested that hemorrhage originates from cracks in the luminal surface [54]. Later, Davis proposed that hemorrhage initiates within the shoulder region, the thinnest portion of fibrous cap, allowing entry of blood from the lumen into the necrotic core, which then comes into contact with collagen fibrils and tissue factor [48]. The most recent hypothesis "Paterson's" supports the rupture of vasavasorum within advanced lesions. This hypothesis was confirmed by Virmani's observations in a series of sudden coronary death cases, where hemorrhage was mostly frequent in ruptured plaques.

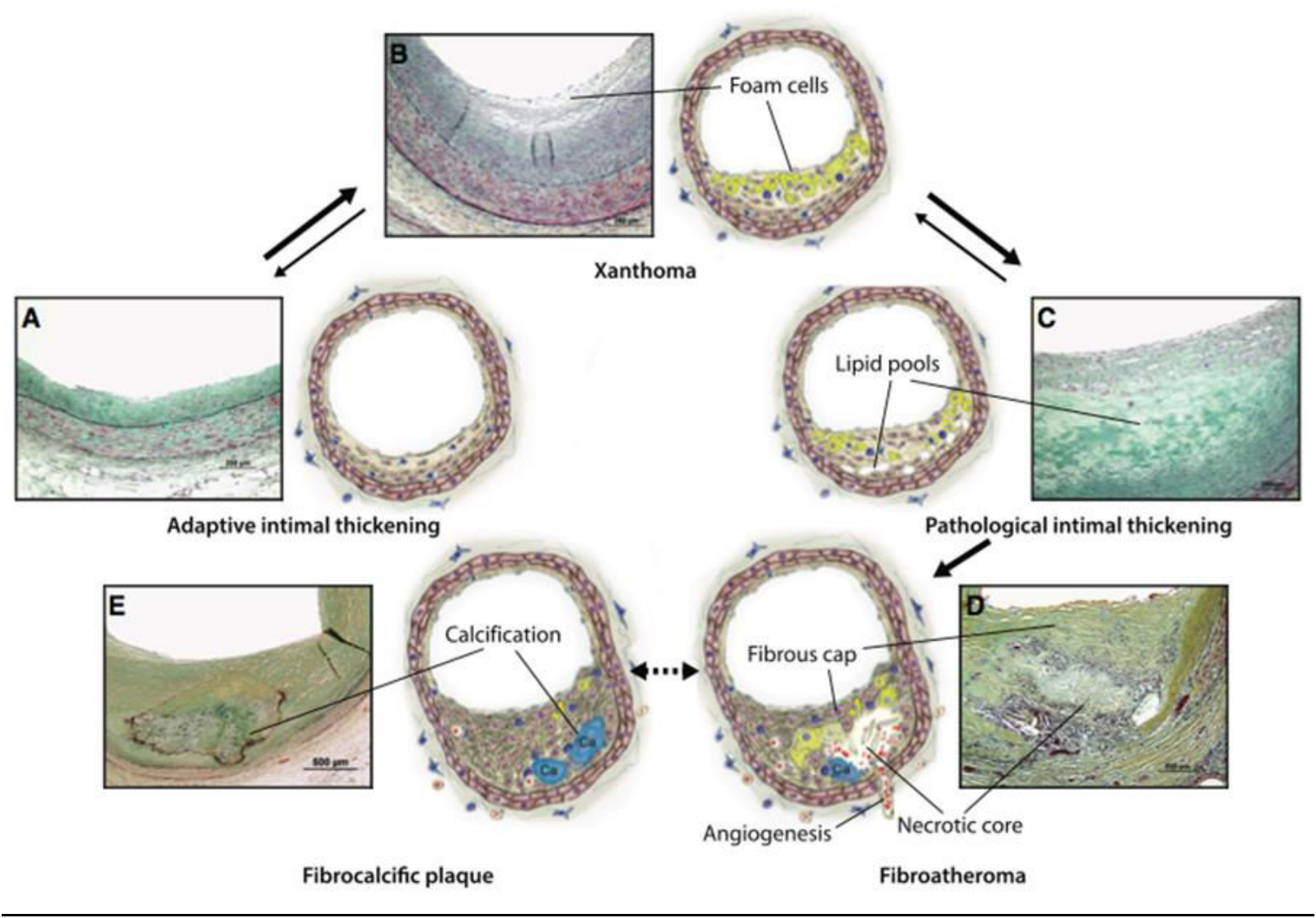

Figure 3: Main lesion types of atherosclerosis and proposed sequence of their development, Movat pentachrome staining of coronary plaques shows collagen and reticular fibers (yellow), fibrin (bright red), muscle (red), elastic fibers (black to blue). A: Adaptive intimal thickening characterized by accumulation of SMCs in the intima. B: Xanthoma corresponds to accumulation of foam cells in the intima, C: Pathological intimal thickening denotes the accumulation of extracellular lipid pools in the absence of apparent necrosis, D: Fibroatheroma indicating the presence of necrotic core, E: Fibrocalcific plaque which results from clacification of the necrotic core and the surrounding tissue. (adapted from Fog Bentzon et al, Circ. Res. 2014) 


\section{Healed Ruptures/Erosions}

Fractured or eroded plaques may heal in the absence of occlusive thrombus and clinical manifestation.

Healed ruptures are characterized by a disrupted fibrous cap filled in by SMCs, proteoglycans, and collagen. The collagen newly synthesized by SMCs is type III replaces the original collagen type I. Lesions may exhibit multilayering of lipid and necrotic core, suggestive of previous episodes of thrombosis [48]. Others show no evidence of a preexisting rupture of the fibrous cap, but instead distinct layers of dense collagen interspersed with SMCs and proteoglycans containing fibrin and platelets.

\section{Compensatory enlargement}

Seymour Glagov showed that in response to plaque growth, the arterial wall can remodel itself by increasing its external diameter to accomodate the plaque without narrowing of the lumen [55]. (Figure 4)

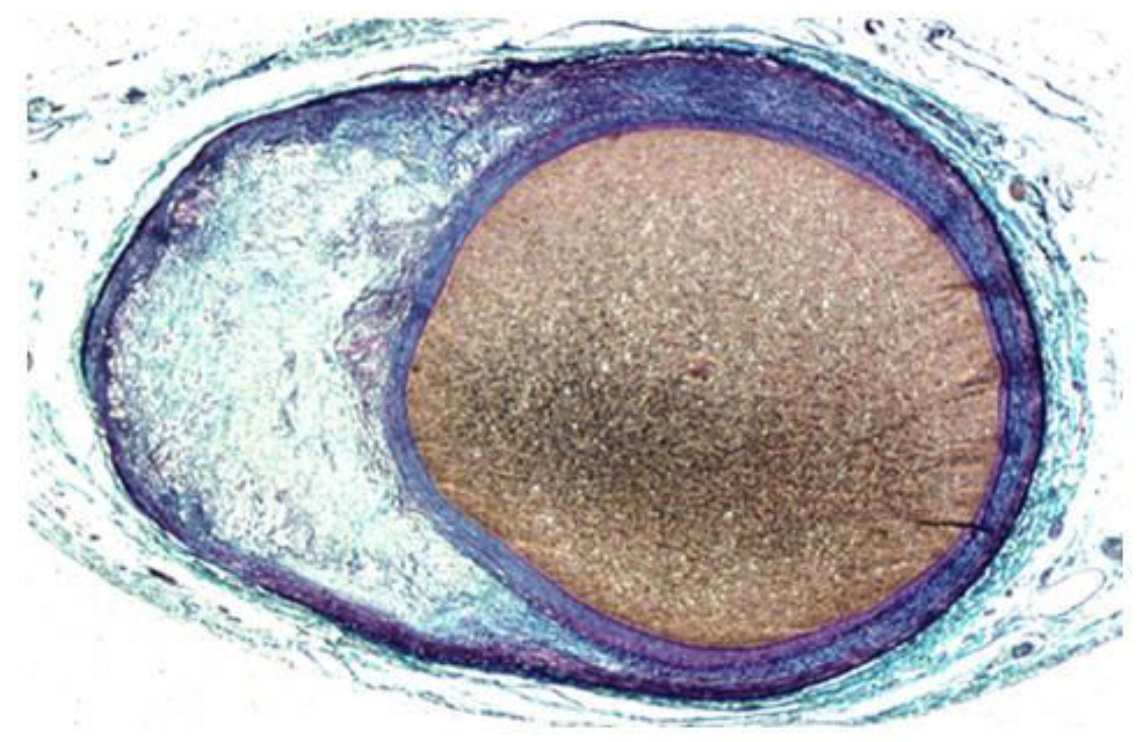

Figure 4: Human Coronary Artery with Compensatory Enlargment, (adapted from S. Glagov, NJEM, 1987) 


\section{E. Animal Models of Atherosclerosis}

Early observations of atherosclerotic lesions in animals date back to more than a century, when Ignatowski found arterial thickening in rabbits fed the lipid rich animal proteins. Since then several animal species were used for atherosclerosis studies; monkey, pig, rabbit, and mice. The most widely used model is the mouse because of the ease of manipulating their genome.

C57BL/6 strain is the most susceptible strain to atherogenic diet, compared to other strains such as BALB/c, SWR, and NZB [56], [57]. However, C57BL/6 mice have low plasma cholesterol levels, mainly in the high-density lipoprotein (HDL), even under high fat diet. So they develop only small lesions when fed an atherogenic diet over a long period [58]. Therefore, transgenic mice on a C57BL/6 background were generated. Among these, the Apoe $^{-/}$and $L d l r^{-/-}$mice are the most commonly used mouse models.

\section{ApoE deficient mice}

ApoE is a $34 \mathrm{KDa}$ glycoprotein synthesized in liver, brain, intestine, lung, and macrophages in both humans and mice [59]. It is a structural component of all lipoprotein particles except low-density lipoproteins (LDL). ApoE functions as a ligand for cell surface lipoprotein receptors that clear chylomicrons and very low- density lipoprotein (VLDL) remnants by the liver [60].

Mice deficient for this glycoprotein (Apoe ${ }^{-/-}$mice) have increased plasma levels of total cholesterol (300 to $500 \mathrm{mg} / \mathrm{dl}$ ), mostly in the VLDL and chylomicron fractions [61]. Apoe $\mathrm{e}^{-/}$ mice are hyperlipidemic even when fed a chow diet [62]. Under normal dietary conditions, $\mathrm{Apoe}^{-/-}$mice have already very high cholesterol levels and develop lipid streaks with foam cells and SMC within 6-8 weeks and more advanced plaques at 20 weeks with necrotic core and fibrous cap [63]. This process can be exacerbated under the influence of western diet (consisting of $21 \%$ fat and $1.5 \%$ cholesterol, similar of everyday diet of western countries) with female mice more sensitive than male mice [64].

The use of Apoe $^{-/-}$mice has some attendant disadvantages. ApoE has several functions in addition to its indispensable role in lipoprotein metabolism. It is implicated in macrophage biology, immune functions, and adipose tissue biology. Morevoer, it is expressed by bonemarrow derived cells which prevents the transfer of Apoe $^{+/+}$bonemarrow into Apoe $\mathrm{e}^{-/-}$ mice because of the correction of cholesterol efflux and normalization of plasma cholesterol concentrations [65]. 


\section{LDL receptor deficient mice}

LDL receptor is a membrane receptor of a molecular weight of $160 \mathrm{KDa}$, which mediates endocytosis of cholesterol rich LDL, and thus maintains plasma level of LDL. It also facilitates the uptake of ApoB- and ApoE- containing lipoproteins [66], [67].

In contrast to $\mathrm{Apoe}^{-/-}$mice, $\mathrm{Ldlr}^{-/-}$mice display modestly elevated plasma cholesterol levels and develop no or only mild atherosclerosis when fed a normal diet. Therefore, these mice are fed western diet to accelerate atherosclerosis. $L d l r^{-/}$mice have increased IDL and LDL sized particles, whereas HDL and triglycerides remain unaffected [68].

$L d l r^{-/}$mice plaques are the same as those seen in Apoe ${ }^{-/-}$mice. The plaque development occurs in a time dependent manner, initially in proximal aorta spreading towards distal aorta. A western diet induces larger and more advanced lesions, with collagen rich fibrous cap, and a necrotic core containing cholesterol and cellular enrichment adjacent to the lumen [69], [70].

The $\mathrm{Ldlr}^{-/-}$mouse model has some advantages compared to the Apoe ${ }^{-/-}$mice. In $\mathrm{Ldlr}^{-/}$mice, plasma cholesterol is mostly carried by LDL particles, which is similar to human lipid profile. Also, the absence of LDL receptor does not have impact on inflammation as compared to ApoE deficiency. Thus atherosclerotic plaque development in this mouse model is due to elevated plasma lipid levels solely and not caused by functions linked to the receptor itself [71]. Moreover, the $\mathrm{Ldlr}^{-/}$mouse model shares the characteristics observed in human familial hypercholesterolemia, which is caused by absence of functional LDL receptor [72], [73], [74]. Finally, Ldlr-positive donors can be used in bonemarrow transplantation experiments, because the receptor rapidly becomes downregulated, even in expressing cells, as plasma cholesterol concentrations increase [65].

In our laboratory, we use in common practice the irradiation/transplantation method starting form $\mathrm{Ldll}^{-/}$mice, making it possible to obtain the so-called chimeric mice. This technique entails the lethal irradiation $(9.5 \mathrm{~Gy})$ of atherosclerosis prone mice to cause medullary aplasia, followed by their reconstitution with the bone marrow (BM) cells of donor mice, which usually present invalidation or overexpression of the gene of interest. This method allows to study specifically the role of a molecule expressed by cells derived from the bone marrow of which leukocytes are a major part. In our studies, the group of mice irradiated and reconstituted with the bone marrow deficient for the gene of interest is always compared to another control group irradiated and reconstituted with controls' bone marrow. This way we would exclude the effect of irradiation on atherosclerosis development, but may not eliminate 
the possible interaction between the gene of interest and the "irradiation factor".

\section{F. Atherosclerosis-a chronic inflammatory disease}

\section{Changes in vessel morphology associated with development of atherosclerotic lesions}

The normal arteries consist of three major layers: tunica intima, media, and adventitia respectively from inside to outside (Figure 5, A). The tunica intima is lined by a monolayer of endothelial cells that is in contact with blood overlying a basement membrane. It is defined as the layer starting from the endothelium and extending to the luminal margin of the media.

Endothelial cells and SMCs are the principal cellular components of the human arterial intima. Isolated macrophages are also present, in addition to sparse mast cells [75].

The endothelium serves as a barrier between circulating molecules and cells in blood. It is a major regulator of vascular homeostasis, by synthesizing signaling molecule, it inhibits platelet aggregation and adhesion, SMC proliferation, and leukocyte adhesion and migration. Structural and functional changes in endothelial cells contribute to the pathogenesis of atherosclerosis.

The sub-endothelial layer of the intima consists of two layers. The inner layer, a proteoglycan layer mainly contains non-fibrous connective tissue, small amounts of elastic fibers, and SMCs of synthetic and contractile phenotype. The musculoelastic layer is a thicker layer, which underlies the proteoglycan layer, contains a large number of SMCs of the contractile phenotype and elastic fibers [75], [76].

In response to growth regulatory molecules such as growth factors, SMC number would increase in the developing intima by mitosis. Originally, it was thought that SMCs in atherosclerotic lesions are solely derived from the media. But, recently, it has been suggested that bone marrow progenitor cells infiltrate the intima and probably differentiate in vivo to form SMCs [77]. Under pro-atherogenic conditions, SMC differentiate in macrophage-like capable of internalizing lipids and transforming into foam cells [78].

Collagen maintains endothelial cell integrity by anchoring endothelial cells to subendothelial matrix. The major types of collagen in the artery wall are the two interstitial collagens, types I and III.

Type III collagen is localized to the subendothelial space of the intima; it could be synthesized by the endothelium. Increased amounts of type I may reflect the metabolic properties of an increased number of SMCs present in the intima, because SMCs in culture 
synthesize both type I and III collagen [75].

The internal elastic lamina, generally considered part of media, denotes the border between intima and media. In areas of vascular transitions, the internal elastic lamina is partly or completely absent and the intima and media may appear as a single unit.

The middle layer, tunica media, is the thickest layer of normal vessels. It contains $20 \%$ of SMCs both contractile and synthetic phenotype and 60\% collagen and elastin, which provides contractile ability for the vessel [79]. By proliferating and migrating to the subendothelial intima, medial SMCs can contribute to the development of atherosclerosis. The outermost layer, tunica adventitia, contains sparse collagen fibrils, nerve endings, and its own nutrient arterial supply calledvasa vasorum. It is separated from the media by the external elastic lamina. The major cell types in the adventitia are sparse fibroblasts and mast cells [75]. This layer can change function and contribute to the development of atherosclerosis. Lymphoid organ like structure are found in the adventitia of human plaques; these are mainly composed of B and T cells [80].

\section{Initiation of atherosclerosis}

In 1976, Ross proposed the response to injury hypothesis, which states that atherosclerotic lesions result as a response to some form of injury to the endothelial cells that result in their desquamation. The loss of the endothelium exposes the underlying collagen layer and SMCs to platelet derived growth factor from platelets that adhere to the exposed subendothelial connective tissue. Infiltration of platelets derived factors such as lipoproteins and hormones would lead to migration and proliferation of SMCs, which form new connective tissue and results in intracellular and extracellular lipid deposition [81].

While the response to injury hypothesis supposed that endothelial desquamation is an essential event in atherogenesis, it was later demonstrated that the developing atheromatous lesions are covered by an intact endothelial layer throughout most stages of lesion progression. Thus in 1995, Kevin Williams and Ira Tabas formulated the "response to retention" hypothesis, which states that extracellular trapping of cholesterol-rich lipoprotein within the arterial intima is sufficient for initiation of atherosclerosis [82].

According to this hypothesis, hypercholesterolemia induces rapid LDL retention followed by their firm adhesion to proteoglycans of extracellular matrix, which prevents their return to the blood stream. Then, LDL are susceptible to enzymatic and non-enzymatic oxidation, as well as modification by extracellular proteins in the subendothelial space [83], [84]. A number of potential oxidant-generating systems have been identified to target lipid and apolipoprotein B 
component of LDL. In addition to metal ions and oxidative radicals, these include: myeloperoxidases, inducible nitric oxide synthase, and 15-lipoxygenase (15-LO) [85]. Once oxidized, LDL exerts various pro-inflammatory biological effects leading to the development of atherosclerotic plaques. The "retention hypothesis" and "inflammatory theory" are complementary, LDL accumation and modifications in the intima being the primary trigger of vascular inflammation.

\section{Endothelial cell activation and Leukocyte recruitment}

In lesion prone areas, atherosclerotic lesions begin to develop under an intact but activated and dysfunctional endothelium [12].

Soon after initiating an atherogenic diet, light microscopy reveals attachment of blood leukocytes to the endothelial cells that line the intima [86], [87].

The normal arterial endothelium does not support the adherence of leukocytes. However, when subjected to irritative stimuli (such as dyslipidemia, hypertension, or pro-inflammatory cytokines), endothelial cells express adhesion molecules that capture leukocytes on their surfaces [76].

Oxidised LDL (oxLDL) activates endothelial cells leading to expression of VCAM-1 and ICAM-1, P- and E- selectins [88].

The recruitment of circulating leukocytes occurs via a tightly regulated multi-step process mediated by a combination of cell surface adhesion molecules.

Initially, activated endothelial cells express E-selectin (CD62E) and P-selectin (CD62P), which mediates the tethering and rolling of the circulating monocytes. P-selectin binds to Pselectin glycoprotein ligand-1 (PSGL1). Although PSGL1 is constitutively expressed by leukocytes, it is functional only when glycosylated correctly. In addition to PSGL-1, Eselectin also binds to glycosylated CD44 and E-selectin ligand 1 (ESL1) [89].

Besides selectins, VCAM-1, a member of immunoglobulin-like superfamily of adhesion molecules participates in the slow rolling and early adhesion of mononuclear leukocytes to the arterial wall by binding to its ligand, Very late antigen-4 (VLA-4), also known as $\alpha 4 \beta 1$ integrin.

VCAM-1 is not routinely expressed under physiological conditions, but is rather induced by cytokine-stimulated endothelium such as Interleukin-1 $\beta$ or tumor-necrosis factor- $\alpha$ (TNF$\alpha)$.

The functional importance of VCAM-1 expression in atherosclerotic lesions is supported by studies demonstrating that monocyte adhesion to endothelial cells of carotid arteries from 
Apoe $^{-/-}$mice was significantly inhibited by antibody blockade of VLA-4 or VCAM-1 [89], [87].

Firm adhesion to the activated endothelium is followed by leukocyte diapedesis between intact endothelial cells and their junctions to penetrate the tunica intima. This migration is directed by a chemoattractant gradient (Figure 5, B).

Several families of chemoattractant cytokines (chemokines) participate in recruiting leukocytes into the arterial intima. Of major importance is the monocyte chemoattractant protein-1 (MCP-1/CCL2), which interacts with its receptor CCR2 (C-C chemokine receptor 2) on monocytes/macrophages. Studies using compound mutant mice lacking CCR2 or MCP1/CCL2 and susceptible to atherosclerosis $\left(\mathrm{Ldll}^{-/-}\right.$or $\left.\mathrm{ApoE}^{-/}\right)$have shown striking decreases in mononuclear phagocyte accumulation and local lipid levels [87], [85]. Cytokines, such as IL-8, may also play a role in macrophage/monocyte trafficking. Also, chemokines, which include trio-IFN- $\gamma$-inducible chemokines of the $\mathrm{CXC}$ (C-X-C motif Chemokine) family; monokine induced by IFN- $\gamma$ (Mig/CXCL9), inducible protein-10 (IP-10/CXCL10), and inducible T-cell a chemoattractant (I-TAC/CXCL11) are selective for lymphocyte recruitment. These bind to the chemokine receptor CXCR3 expressed by $\mathrm{T}$ cells in the atherosclerotic lesion [90].

\section{Amplification of the inflammatory response and lesion progression}

Once resident in the arterial intima, monocytes acquire characteristics of tissue macrophages [76]. Monocytes increase the expression of scavenger receptors such as scavenger receptor A and CD36, and then internalize lipoprotein particles modified by glycation or oxidation [87]. This gives rise to arterial foam cell, the hallmark of early atheromatous precursor, which results from accumulation of lipid droplets within the cytoplasm [91].

Oxidized LDLs are internalized by macrophages. In contrast to the LDL receptor whose expression is down-regulated when intracellular cholesterol content increases, scavenger receptor expression is not downregulated in response to increased modified LDL. Therefore, continuous uptake of oxidized LDL accounts for foam cell formation [92], [93].

Within the intima, the foam cell serves many functions related to complication of atherosclerosis. Notably, foam cells secrete proinflammatory cytokines and reactive oxygen species that amplify local inflammatory response in the lesion.

Lesion progression results from accumaltion of macrophages and is accompanied by migration of SMCs from the media to the intima. Intimal SMCs as well as the medial derived SMCs would proliferate, some of them taking up modified lipoproteins, contribute to foam 
cell formation and synthesize extracellular matrix proteins such as collagen, elastin, and proteoglycans that lead to the development of fibrous cap [8]. Significant distinctions in gene expression and modes of lipid loading between macrophages and SMCs exist. VSMC derived-macrophage-like cells have reduced phagocytic capacity and thus reduced ability to clear lipids, dying cells, and necrotic debris, which would exacerbate inflammation [94], [78]. Plaque macrophages die in advancing lesions, some by apoptosis. Extracellular lipids derived from dead and dying cells accumulate in the central region of a plaque, often denoted the lipid or necrotic core, contributing to the growth of the atherosclerotic plaque [76] (Figure 5, C).
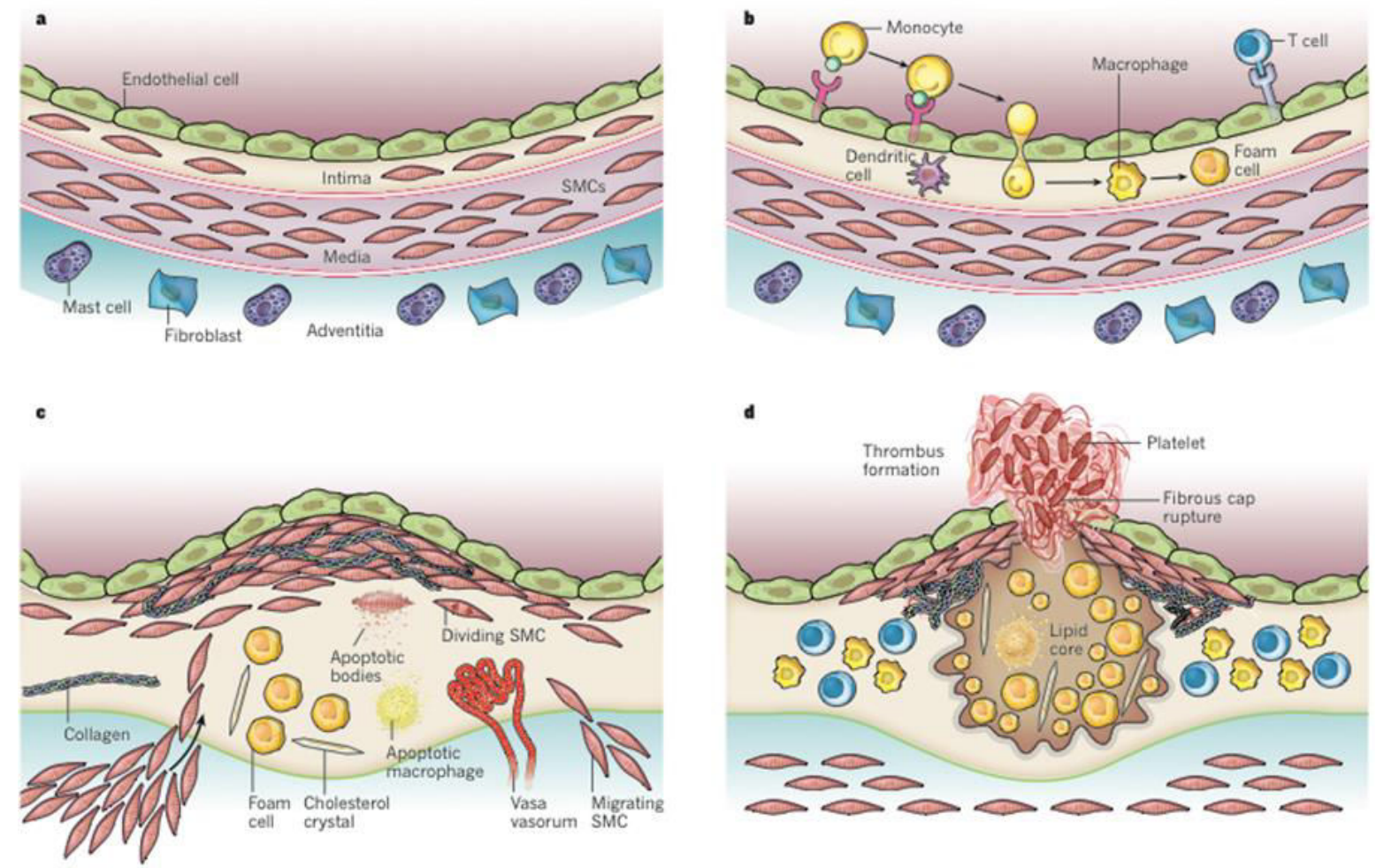

Figure 5: Stages in the development of atherosclerotic lesions, (A) The normal artery contains 3 layers: tunica intima, media, adventitia. (B) The initial steps of atherosclerosis development include: adhesion of leukocytes to the activated endothelial monolayer, migration into intima, maturation into macrophages and their uptake of foam cells. (C) Lesion progression marked by migration and proliferation of SMCs. (D) Thrombosis, or lesion complication. (adapted from Libby, 2011, Nature)

In addition to secreting growth factors for SMCs, inflammatory cells residing in the plaque including macrophages, elaborate MMPs, and produce angiogenic mediators such as acidic 
and basic fibroblast growth factor. Microvessels in plaques may not only serve as site for haemorrhage but may also perform nutritive function promoting plaque growth.

If inflammatory conditions prevail and risk factors such as dyslipidemia persist, the lipid core can grow and MMPs secreted by activate macrophages and vascular cells can degrade the extracellular matrix, while proinflammatory cytokines such as IFN- $\gamma$ secreted can limit the synthesis of new collagen. These changes contribute to thinning of the fibrous cap and render it friable and susceptible to rupture. When the plaque ruptures, blood coming in contact with the Tissue factor expressed by macrophages and leukocyte-derived microparticles in the plaque coagulates.

Platelets activated by thrombin generated from the coagulation cascade and by contact with the intimal compartment instigate thrombus formation. If the thrombus occludes the vessel persistently, an acute myocardial infarction can result [87] (Figure 5, D).

\section{Role of innate and adaptive immune cells in atherosclerosis}

The defense of the normal artery depends on innate immune responses mounted by the endothelial cells (ECs) and after inflammatory challenge by macrophages and other immune cells recruited to the artery wall [95]. Although components of the adaptive immunity are not required for initiation, adaptive immune cells have a major role in progression and complications of the disease. The role of most cells of the innate and adaptive immunity is discussed below in details (Figure 6).

\section{a. Innate immune cells}

- Monocytes

Under homeostatic conditions, monocytes circulate in blood, bone marrow and spleen. These cells are short lived, and do not proliferate in blood. They are phagocytic and can develop into macrophages and dendritic cells (DCs) in vivo and in vitro.

During inflammation, blood monocytes migrate to lymphoid and non-lymphoid tissues, in response to tissue-derived signals such as infection or tissue damage [96].

During the earliest phase of atherogenesis, blood derived monocytes home to the intima and subintima where they differentiate into macrophages or even DCs.

Monocytes are already visible in the fatty streak, the earliest lesion in human and experimental atherosclerosis [97]. Systemic depletion of circulating monocytes by clodronate significantly reduces plaque formation, pointing to their importance in atherogenesis [98]. Also, Osteopetrotic mice (op/op), deficient in circulating monocytes, tissue macrophages and 
osteoclasts, are highly protected from atherosclerosis in the setting of hypercholesterolemia [99].

In mice, monocytes can be defined by their expression of Ly6C, an epitope of Gr-1. Classical Ly6 ${ }^{\text {high }} \mathrm{Gr}-1^{+}$monocytes are $\mathrm{CCR} 2^{\text {high }} \mathrm{CX} 3 \mathrm{CR} 1^{\text {low }}$, extravasate to tissues and mediate inflammation, phagocytosis, and proteolysis. However, non-classical Ly $6 \mathrm{C}^{\text {low }} \mathrm{Gr}-1^{-}$ monocytes defined as $\mathrm{CCR} 2^{\text {low }} \mathrm{CX} 3 \mathrm{CR} 1^{\text {high }}$, mediate wound repair, tissue remodeling and expression of chemokines [100].

Under basal conditions, normal mice have few circulating monocytes evenly distributed between $\mathrm{Ly}_{6 \mathrm{C}}{ }^{\text {hi }}$ and $\mathrm{Ly} 6 \mathrm{C}^{\text {lo }}$ populations. Hypercholesterolemia induces monocytosis in the bonemarrow, blood, and spleen with a preferential and dramatic increase in Ly6c $\mathrm{c}^{\text {high }}$ monocytes. In Apoe ${ }^{-/-}$mice, the numbers of circulating monocytes is $50 \%$ higher than in wild type mice [101], [102]. One mechanism could be that cholesterol enrichment of hematopoeitic stem and progenitor cells increases their expression of IL-3 and GM-CSF receptor, and thus hematopoietic stem cell (HSC) proliferation [103].

Ly6C ${ }^{\text {high }}$ monocytes home to atherosclerotic lesions in a manner dependent on CCR2 and CX3CR1, while Ly6c ${ }^{\text {low }}$ monocytes infiltrate lesions via CCR5.

Our laboratory has shown that combined inhibition of CCL2, CX3CR1, and CCR5 in Apoe - $^{-1}$ mice abrogates bone marrow monocytosis and reduces circulating monocytes despite persistent hypercholesterolemia. These effects were associated with marked additive reduction in atherosclerosis, and interestingly, the lesion size was highly correlated witht the number of circulating monocytes [104].

Ly6C $\mathrm{C}^{\text {high }}$ subpopulation exhibits series of functions, which render them particularly pathogenic in the context of atherosclerosis. For example, they bind with high avidity to endothelial monolayers. As mentioned earlier, PSGL-1 mediates adhesion and transmigration of monocytes through binding to E- and P-selectins of the activated endothelium. It is worth noting that Ly6C $\mathrm{C}^{\text {high }}$ monocytes express higher levels of PSGL-1 than Ly6C ${ }^{\text {low }}$, which suggests its selective recruitment to the developing lesion [100].

Also, Ly6C high monocytes express high amounts of pro-inflammatory cytokines and proteases implicated in the pathogenesis of atherosclerosis than their Ly6C ${ }^{\text {low }}$ counterparts. Ly6 $\mathrm{C}^{\text {high }}$ monocytosis is induced not only in peripheral blood but also in spleen. Proinflammatory monocytes of splenic origin comprise up to a quarter of the mononuclear phagocytes in mouse atheromata [105], [106].

However, it remains unknown whether foam cells arise from either differentiated Ly6C ${ }^{\text {high }} \mathrm{Gr}-1^{+}$or Ly6 $\mathrm{C}^{\text {low }} \mathrm{Gr}-1^{-}$monocytes. 

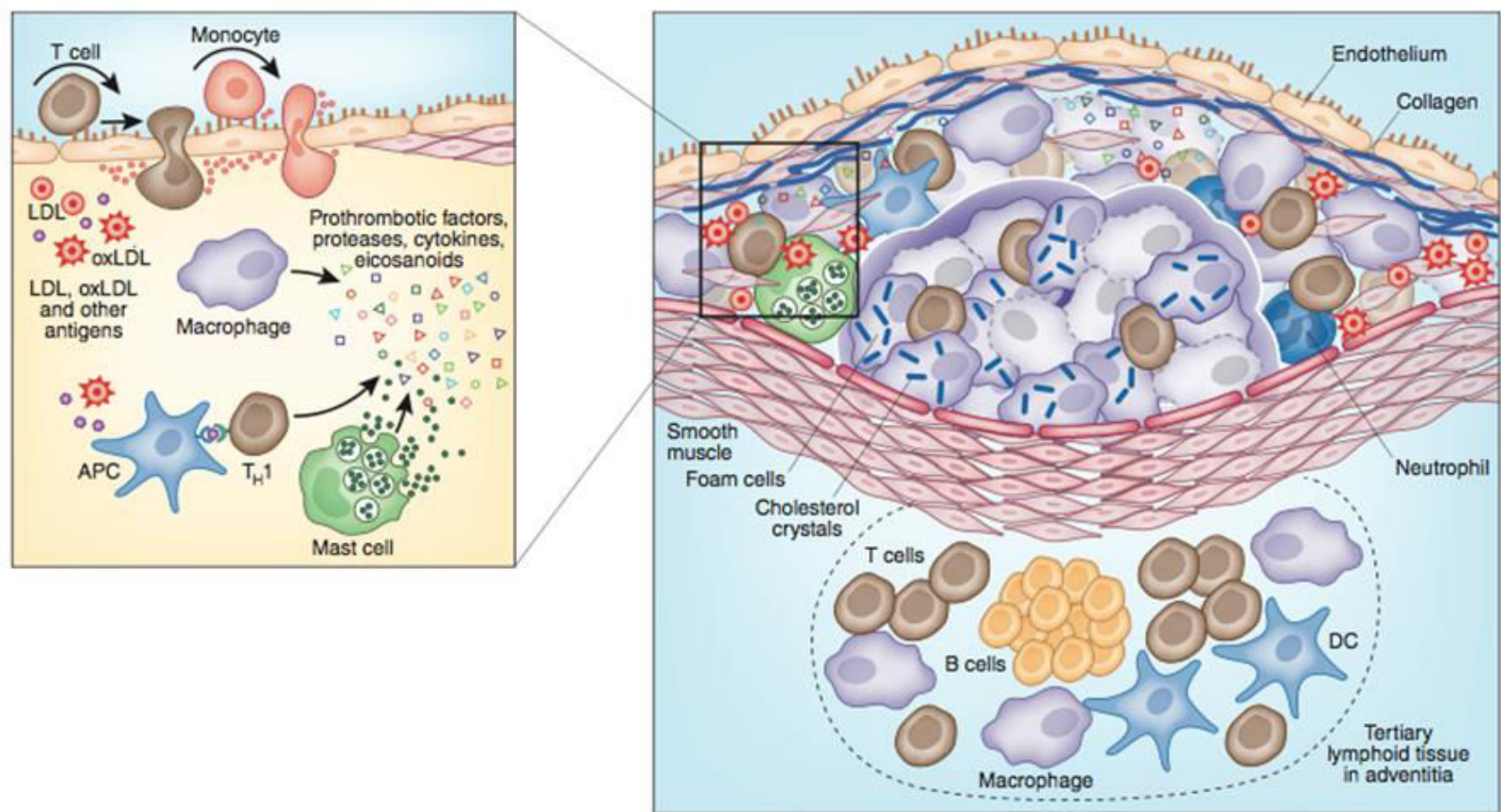

Figure 6: Immune components of the atherosclerotic plaque, Several types of cells of the immune response are present in the atheroma; these include: macrophages, $\mathrm{T}$ cells, dendritic cells, and mast cells. Cells may accumulate in the adventitia outside the atheroma where they develop into tertiary lymphoid structures with germinal centers. (adapted from Hannson and Hermanson, 2011, Nature)

\section{- Macrophages}

The first demonstration of a major role of macrophages in atherosclerosis was the study whereby macrophage colony-stimulating factor (op) and apolipoprotein E deficient mice had significant decrease in plaque size compared to control Apoe ${ }^{-/-}$despite high cholesterol levels [99].

Once in the arterial wall, monocytes differentiate into macrophage under the action of colony stimulating factor (M-CSF) produced by activated endothelial cells and SMCs. Intimal macrophages take up lipids through their scavenger receptors. Numerous scavenger receptor family members including SR-A1, SR-A2, SR-B1, CD36, LOX-1, and SREC1 can bind oxidized LDL and promote lipid-laden foam cells formation, the hallmark of atheromata [107].

SR-A1 and CD36 receptors mediate $75-90 \%$ of the degradation of acetylated or oxidized LDL in vitro. Combined deficiency of SR-A1 and CD36 reduced atherosclerosis in Apoe-/mice. Plaques in these mice have reduced inflammation, macrophage apoptosis, and secondary necrosis, which suggests that these mice have roles beyond lipid uptake [108], [109]. 
Scavenger receptors internalize oxLDL, where cholesteryl esters of the lipoproteins are hydrolyzed to free cholesterol and fatty acids in the late endolysosomal compartment. Free cholesterol is then trafficked to the endoplasmic reticulum ER where it undergoes reesterification by ACAT1 (acetyl-co-enzyme A: cholesterol acetyl-transferase 1) to cholesterol fatty acid esters that provide the "foam" of foam cells [107].

In advanced plaques, macrophages continue to be major contributors to the inflammatory response through their secretion of pro-inflammatory mediators (including chemokines, cytokines, and reactive oxygen and nitrogen species) and MMPs, and through their eventual death by necrosis or apoptosis. Increased macrophage apoptosis combined with defective efferocytosis results in secondary necrosis and in release of cellular components and lipids that form the necrotic core [110].

Functionally, like monocytes, macrophages are of two subsets. M1 macrophages, highly inflammatory, are classically activated by stimulation with IFN- $\gamma$, LPS, or GM-CSF. These inflammatory macrophages produce high levels of IL-2, TNF- $\alpha$, IL-1 $\beta$, IL-6, and iNOS (inducible nitric oxide synthase). M2 macrophages are alternatively activated upon culturing with IL-4, IL-10, and IL-13. They do express anti-inflammatory arginase 1, IL-10, and CD206 [111].

Both M1 and M2 macrophages are present in human lesions and macrophage phenotypic polarization correlates with lesion progression. The intraplaque cytokine milieu dictates macrophage differentiation into the M1 (pro-inflammatory) or M2 (reparative) phenotype. A large body of literature supports predominance of the M1 subpopulation of macrophages in atheromata [106]. These macrophages elaborate mediators associated with the progression and complication of atherosclerosis. Their less inflammatory counterparts M2 elaborate mediators that lessen atherosclerosis and resolve plaque inflammation. In Apoe ${ }^{-/-}$mice, M1 subset dominated over M2 in disease progression. However, in regression studies, the proportion of M1 to M2 macrophages is decreased [112].

\section{- Dendritic cells}

DCs are professional antigen presenting cells (APCs) that recognize foreign antigens and present them bound to MHC molecules on their surface. These cells home preferentially to T-cell rich- lymphoid organs where they present antigen to naïve T cells [113].

DCs belong to the myeloid lineage of blood cells. In mice, DCs are characterized by the expression of CD11c, and inducible expression of MHC II, CD80, CD86, and CD40. However, certain surface markers are shared with tissue macrophages, such as CD80 and 
CD86 expression [114], [115]. As mentioned earlier, circulating monocytes recruited to the tissue can differentiate into either macrophages or DCs [116]. DCs were identified in both human and mouse atherosclerotic lesions [117], [118]. In the normal artery, DCs reside in the subendothelial space of tunica intima and tunica adventitia where they can mediate early recognition of danger signals.

Resident DCs are thought to promote tolerization to antigen either by silencing $\mathrm{T}$ cells or expanding regulatory $\mathrm{T}$ cells. However, danger signals generated during atherogenesis may activate DCs, leading to a switch from tolerance to activation of adaptive immunity [119], [120].

Studies depleting resident intimal DCs in $\mathrm{Ldll}^{-/-}$mice using CD11c-DTR model resulted in reduced intimal lipid surface areas and foam cells, suggesting that resident intimal DCs differentiate into initial foam cells in emerging atherosclerotic lesions [121].

Moreover, in a mouse model overexpressing the apoptosis inhibitor (Bcl-2) under the control of CD11c specific promoter, increases in DC population lead to severe decrease in plasma cholesterol level in both $L d l r^{-/}$and Apoe ${ }^{-/-}$mice. Although $\mathrm{T}$ cell activation as well as a shift towards TH1 cells was observed, there was no change in atheroma. While TH1 activity is thought to promote atherosclerosis, the result observed was possibly due to reduction in cholesterol due to DC expansion. The authors suggested that conventional DCs contribute to correction of hyperlipidemia and regulation of cholesterol possibly via lipoprotein uptake or clearance of cholesterol from the circulation [122].

All of these studies focused on the myeloid CD11c+ DCs. However, concerning the plasmacytoid DC (pDCs) population, studies entailing their relevance to atherosclerosis have been contradictory, certain suggesting atherogenic, others; anti-atherogenic role. pDCs are specialized type I interferon (IFNs) producers in response to virus infection, and thus are major players in innate immune responses [123].

One study demonstrated that targeted depletion of pDCs by $120 \mathrm{G} 8 \mathrm{mAb}$ increased atherosclerotic lesions along with increased $\mathrm{T}$ cell accumulation in plaques and elevated IFN$\gamma$ production, and thus pointed out to an atheroprotective role of this subset [124]. On the contrary, our laboratory reported a proatherogenic role for pDCs by presenting MHC IIrestricted antigen to $\mathrm{T}$ cells and driving proatherogenic $\mathrm{T}$ cell immunity, using selective mouse model of pDC deficiency (CD11c-CreXTcf4- ${ }^{\text {lox }}$ ) [123].

These findings imply that different subsets of DCs may have different stimulatory or regulatory functions in atherosclerosis. 


\section{- Mast cells}

Mast cells do accumulate in human atherosclerotic plaques, particularly at sites of plaque rupture. These are major effectors in allergy and defense against parasites, and produce TNF$\alpha$, proteinases and MMPs, which could contribute to atherogenesis. Signals from eotaxin, a chemoattractant, which interacts with the chemokine receptor CCR3 attracts mast cells to the intima. Once in the intima, they undergo degranulation releasing preformed TNF- $\alpha$, heparin with its anti-coagulant effects and inhibitory effects on SMCs, and serine proteases tryptase and chymase, which would activate MMPs [30].

Recent evidence suggests that mast cells play an important role in plaque progression and instability by increasing intraplaque hemorrhage, macrophage apoptosis, leukocyte recruitment, and vascular leakage [125], [88].

\section{- NK cells}

The role of Natural killer cells in atherosclerosis will be discussed in the next chapter.

\section{b. Adaptive Immune cells}

The first suggestion of an implication of adaptive immunity came from observations that human-meukocyte-antigen (HLA)-DR was abundantly expressed in atherosclerotic lesions. Then, in 1980's, it was reported that autoantibodies to oxLDL are generated. This was followed by the discovery that $\mathrm{CD} 4+\mathrm{T}$ cells from human atherosclerotic lesions recognize LDL-derived antigen in HLA-DR-dependent manner [123].

\section{- Lymphocytes}

Immune activation is ongoing in atherosclerotic lesions. Although lymphocytes are not required for the development of atherosclerosis, their intervention modulates the progression of the disease.

Whereas macrophages comprise the vast majority of inflammatory cells in human and experimental atherosclerotic plaques, the cells of adaptive immunity, B and T lymphocytes are also present, albeit in markedly lower numbers [126].

\section{- CD4+ T cells}

In healthy arteries, $\mathrm{T}$ cells are normal residents of the adventitia. However, in atherosclerosis prone-areas, $\mathrm{T}$ cells are recruited to the intima in parallel with macrophages by similar mechanisms involving chemokines and adhesion molecules. They are not as abundant, with 
an approximate macrophage/T cell ratio of 4:1 to 10:1 in human lesions [127], [128].

Naïve $\mathrm{T}$ cells are rarely found in nonlymphoid tissues. This holds true for $\mathrm{T}$ cells of the atherosclerotic plaque, which are of the effector or memory phenotype [129].

Earlier studies demonstrated pathogenic role for CD4+ T cells in atherosclerosis. Transfer of CD4+ T cells into Apoe ${ }^{-/-}$SCID mice (severe combined immunodeficiency) accelerated atherosclerosis, with homing of $\mathrm{T}$ cells into the lesions [130]. Another study demonstrated that depleting CD4+ $\mathrm{T}$ cells by a specific antibody reduced fatty streak development in C57BL/6 mice on an atherogenic diet [131].

$\mathrm{T}$ cells can be activated by antigens within the atherosclerotic lesions, including oxLDL, $\beta 2$ glycoprotein I ( $\beta 2 \mathrm{GPI})$, and the microbial heat-shock protein (HSP) 65 [132].

Mature CD4+ $\mathrm{T}$ cells can be divided into three different subpopulations, $\mathrm{TH} 1, \mathrm{TH} 2$, and TH17 subsets. These are distinguished by their differential cytokine profile and thus can influence atherosclerosis differently (Figure 7).

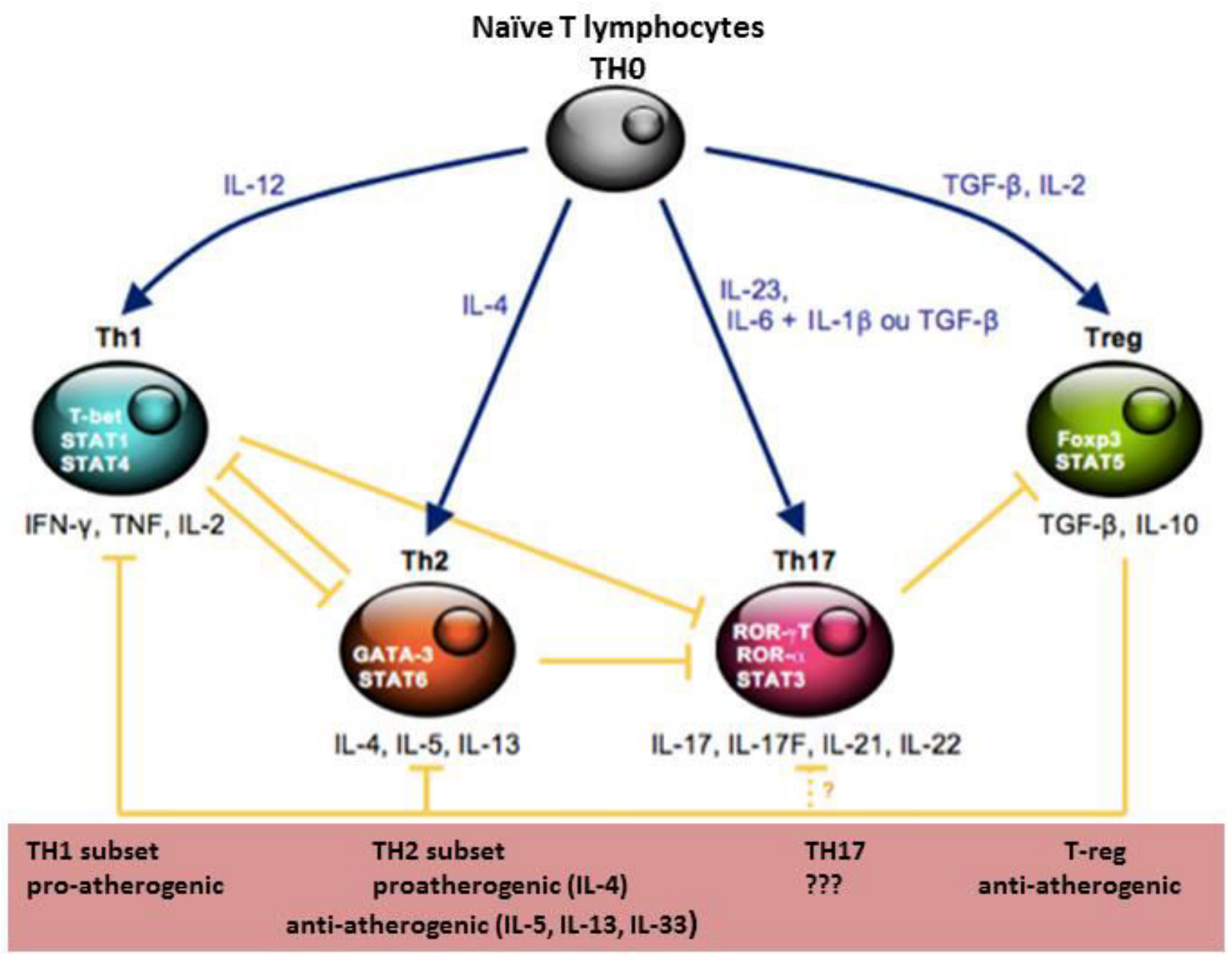

Figure 7: Role of different CD4 $T$ cell subsets in atherosclerosis, (modified from Aitoufella et al, Immunol, 2009) 


\section{- TH1 cells}

Atherosclerosis is driven by $\mathrm{T}$ helper type 1 (TH1) response. These are activated in the plaques, express several adhesion molecules, secrete cytokines such as IFN- $\gamma$, TNF- $\alpha$, and IL-2, and may proliferate in situ.

The signature TH1 cytokine is IFN- $\gamma$, which exerts several proatherogenic actions. These include activation of macrophages and DCs, which improve the efficiency of antigen presentation and promotes further TH1 polarization. The activated macrophages would in turn produce numerous inflammatory and cytotoxic molecules such as TNF- $\alpha$ and IL-1, which act on ECs and SMCs. This cascade of cytokines would stimulate the release of IL-6, which induces the production of acute phase reactants such as fibrinogen and C-reactive protein [133]. In addition, IFN- $\gamma$ pathogenic effects include inhibition of collagen synthesis and SMC contractility. Hence, IFN- $\gamma$-producing TH1 cells would promote plaque rupture and destabilization [127].

Mice deficient in IFN- $\gamma$ or its receptor both have a lower lesion burden and injections of IFN$\gamma$ increase lesion size as compared to controls [134], [135].

In addition, mice lacking IL-12 or IL-18, TH1 promoting cytokines, show reduced atherosclerosis development, whereas, injections of IL-12 or IL-18 promote lesion formation [136], [137], [138], [139]. IL-12, a pro-inflammatory interleukin, is mainly produced by the macrophages, B cells, and DCs. It acts in synergy with IL-18 for the production of IFN- $\gamma$ by T cells. IL-18, also known as IFN- $\gamma$ inducing factor, is another pro-inflammatory interleukin, with multiple biological functions. It is mainly produced by macrophages [140].

Furthermore, targeted deletion of Tbx21, the gene encoding TH1-differentiating transcription factor T-bet, leads to much less lesion development in $\mathrm{Ldll}^{-/}$mice and switching towards TH2 phenotype [141]. Collectively, these data demonstrate that TH1 cells have major role in development of atherosclerosis.

\section{- TH2 cells}

TH2 cells produce IL-4, IL-5, IL-10, and IL-13 cytokines, which are known to downregulate TH1 responses (notably their IFN- $\gamma$ production) and support antibody production by B cells. IL-4, the signature cytokine of $\mathrm{TH} 2$ lineage is not frequently observed in human plaques [142].

TH2 might counter the proatherogenic effect of TH1 cells. The role of TH2 cells in atherosclerosis development remains unclear. It depends on the stage and arterial site of the 
disease, and cytokines produced. IL-5 production is necessary for the production of protective antibodies against oxLDL while IL-4 production can induce proatherogenic effects [133].

\section{- TH17 cells}

In addition to the signature cytokine IL-17A, TH17 cells also produce IL-22 and IL-23. Differentiation of naïve T cells into TH17 is induced by nuclear orphan receptor ROR- $\gamma \mathrm{T}$. IL-17 inhibits TH1 polarization, IFN- $\gamma$ production, and T-bet expression [143].

TH17 and its cytokine IL-17 have been identified in murine and human plaques. However, their role in atherosclerosis development is controversial. Earlier studies suggested proatherogenic role in a double Apoe and Fc $\gamma$ chain deficient mouse model. These mice had less atherosclerosis with reduced TH17 numbers and increased numbers of Tregs [144].

On the contrary, our laboratory demonstrated that depletion of IL-17 with an anti-IL17 antibody accelerated atherosclerosis development and increased IL-17 production was associated with reduced atherosclerosis [145].

Moreover, the atheroprotective effect of B cell depletion was accompanied with increased IL-17 production. These experiments implied an atheroprotective effect for TH17 [146].

\section{- Treg cells}

Tregs are known as modulators of the immune response where they maintain the balance of immune effector cells, TH1 and TH2, and ensure self-tolerance. Their immune suppressive actions are mediated either by production of cytokines, TGF- $\beta$, IL-10, and IL-35, and consumption of IL-2 growth factor or through cellular contact; CTLA4 on Treg with B7 on APCs and T cells [147].

Human atherosclerotic lesions contain only limited Treg numbers (1 to $5 \%$ of all $\mathrm{T}$ cells) [148].

Patients with CAD have reduced Treg numbers as well as compromised functional properties. Apoe ${ }^{-/-}$mice have less Tregs in the spleen and impaired Treg suppressive function. These studies suggest that Treg-mediated protection by immune tolerance is hampered in atherosclerosis [149], [150].

Indeed, accumulating evidence from mouse studies point to a protective role of Tregs in atherosclerosis. Genetic inactivation or blockade of TGF- $\beta$ or IL-10 by neutralizing antibody accelerated lesion development by enhancing TH1 and TH2 responses [151], [152], [153]. 
Also, depletion of Treg cells in mice models with deletion of CD80/CD86, CD28, ICOS or after treatment with CD25 neutralizing antibodies, had increased atherosclerotic burden [154], [155].

In addition to their suppressive functions on other effector $\mathrm{T}$ cells, Treg can directly regulate endothelial cell activation and leukocyte recruitment [156]. Also, they can inhibit the proinflammatory phenotype of oxLDL-induced macrophages resulting in reduced foam cell formation and differentiation of macrophages towards an anti-inflammatory phenotype [157].

\section{- CD8+ T cells}

Little data exist regarding the precise role of CD8+ T cells in atherosclerosis. Apoe ${ }^{-/-} \mathrm{CD}^{-/-}$ mice exhibit no change in lesion development compared to Apoe ${ }^{-/}$mice.

However, CD8+ T cells stimulated by injection of an agonist to the TNF- $\alpha$ like surface protein $\mathrm{CD} 137$ or activated to an artificial antigen expressed by SMCs increase atherosclerosis in Apoe ${ }^{-/-}$mice [158], [159].

CD8+ T cells might be controlled by PD-1 (programmed death-1), since $L d l r^{-/-}$mice deficient in PD-L1 (programmed death ligand-1), and PD-L2 have larger plaques with massive infiltration of CD8+ T cells. PD-L1 and PD-L2 are B7 family members expressed on several cell types, which inhibit T cell activation via binding to PD-1 on T cells [160].

Proteins of bacterial or viral origins are candidate antigens of CD8 T cells. Accordingly, it has been reported that patients with coronary artery disease had an expansion of CD8+ T cells associated with cytomegalovirus infection [161]. Thus, in a situation where an intracellular antigen activates CD8 cells, as in the case of viral infection, CD8+ T cells might promote atherosclerotic lesion formation.

\section{- Natural Killer T (NKT) cells}

These are distinct subset of $\mathrm{T}$ cells expressing both $\mathrm{T}$ cell (TCR) and natural killer cell markers, CD161 in humans and NK1.1 in mice. NKT cells recognize lipid antigens presented by hydrophobic MHC-like molecule, CD1d expressed on APCs [162]. The major subset of NKT cells, also known as invariant (iNKT), utilizes invariant V $\alpha 14-\mathrm{J} \alpha$ 18 $\alpha$-chain paired with $\mathrm{V} \beta 8$ or Vb2 $\beta$-chain. NKT cells can modulate the immune response by secreting TH1 and TH2 cytokines, cytotoxic molecules, and supporting B cell responses [163].

NKT cells increased in blood and spleen of Apoe ${ }^{--}$mice treated with LPS associated with 
increase in lesion size. Later studies attempting at depletion of NKT cells in a mouse model of CD1d deficiency (which ensures NKT chronic deficiency) or acute activation of NKT cells with glycolipid $\alpha$-galactosylceramide in $\mathrm{Apoe}^{-/-}$or $\mathrm{Ldll}^{-/}$mice confirmed its proatherogeenic role [164], [165], [166], [167].

Moreover, adoptive transfer of iNKT into immune deficient (for $\mathrm{B}$ and $\mathrm{T}$ cells), atherosclerosis susceptible $\mathrm{RAG}^{-/-}$Apoe $^{-/-}$mice increased lesion size enormously compared to mice which received NKT cell deficient splenocytes. This result demonstrates that NKT cells are proatherogenic in the absence of exogenous stimulus, may be due to their constitutive activation by endogenous lipid antigens [168].

\section{- B cells}

B cells have been detected in atherosclerotic lesions, mostly localized to the adjacent adventitia. Splenectomy aggravates atherosclerosis in Apoe $^{-/-}$mice, whereas adoptive transfer of splenic B cells from atherosclerotic $\mathrm{Apoe}^{-/}$mice had a protective effect on the splenectomized recipients[169]. This protective effect could be mediated by IgM, as transfer of B cell-deficient for serum IgM bone marrow into $\mathrm{Ldll}^{-/}$exacerbated atherosclerosis [170].

Accordingly, chimeric $L d l r^{-/}$lacking IL-5, a cytokine that promotes expansion of B-1 cell population, had lower concentration of immunoglobulin $\mathrm{M}$ ( $\operatorname{IgM}$ ) antibodies to phosphocoline and developed more atherosclerosis [171].

B cells appear to be protective by producing IgM Abs to oxLDL. Antibodies against oxLDL in experimental studies where mice and rabbits were immunized with oxLDL have shown positive correlation with the degree of protection against atherosclerosis [172]. In parallel with these studies, infusion of anti-LDL decreased atherosclerosis in hypercholesterolemic mice.

On the other hand, depleting mature B cells with anti-CD20 antibody prevented atherosclerotic lesion development. This was associated with reduced $\mathrm{T}$ cell activation, and diminished $\mathrm{T}$ cell derived IFN- $\gamma$ secretion, and enhanced IL-17 production [146]. Another study demonstrated that adoptive transfer of B-2 cell subset, but not B-1, into lymphocyte (B, $\mathrm{T}$, and NK) deficient Apoe ${ }^{-/-} \operatorname{Rag}^{-/-}$(IL-2R common $\gamma$ ) $\gamma \mathrm{c}^{-/-}$mice aggravated atherosclerosis [173]. These studies suggest that certain subsets of B cells exert contrasting effect on the disease. 
The studies on the role of innate and adaptive immune cells in atherosclerosis development discussed above are summarized in the following table (Table 2).

\begin{tabular}{|c|c|c|}
\hline Cell Subset & Suggested Role & Description \\
\hline Macrophages & Pro-atherogenic & $\begin{array}{l}\text { Monocyte colony stimulating factor (op) and Apoe deficient } \\
\text { mice had a significant decrease in plaque size compared to } \\
\text { control Apoe mice [99]. }\end{array}$ \\
\hline Dendritic cells & $\begin{array}{l}\text { Conventional DCs: } \\
\text { Controversial } \\
\text { Plasmacytoid DCs: } \\
\text { Controversial }\end{array}$ & $\begin{array}{l}\text { Depleting myeloid resident intimal DCs in } \mathrm{Ldll}^{-/} \text {mice using } \\
\text { CD11c-DTR model resulted in reduced intimal lipid surface } \\
\text { areas and foam cells, but did not reduce development or } \\
\text { progression of atherosclerosis [122]. } \\
\text { Targeted depletion of pDCs by 120G8 mAb increased } \\
\text { atherosclerotic lesions [124]. } \\
\text { Mice deficient in CD11c-Cre x Tcf4-flox (selective } \\
\text { deficiency in pDCS) as well as MHC II pDCS had reduced } \\
\text { atherosclerosis compared with controls. MHC II-restricted } \\
\text { antigen presentation by pDCS drives proatherogenic T cell } \\
\text { immunity [123]. }\end{array}$ \\
\hline Mast cells & Pro-atherogenic & $\begin{array}{l}\text { Mast cells play an important role in plaque progression and } \\
\text { instability by increasing intraplaque hemorrhage, } \\
\text { macrophage apoptosis, leukocyte recruitment, and vascular } \\
\text { leakage [30]. }\end{array}$ \\
\hline NK cells & Controversial & $\begin{array}{l}\text { Beige mice fed a high fat diet containing cholate exhibited } \\
\text { no difference in lesion size to control mice [378]. } \\
\text { Beige mice inbred to } L d l r^{-1}\left(L y s t^{\text {beige }} L d l r^{-1}\right) \text { mice showed } \\
\text { significant increase in lesion size compared to control } L d l r^{-1-} \\
\text { mice fed a high-fat diet [379]. } \\
\text { Smaller size lesions were observed in } L d l r^{-/} \text {recipients } \\
\text { reconstituted with the BM of Ly } 49 \mathrm{~A} \text { transgenic mice } \\
\text { compared to those reconstituted with control BM [372]. } \\
\text { Depletion of NK cells with anti-asialo-GM-1 antibody } \\
\text { significantly reduced atherosclerotic lesions compared to } \\
\text { control mice treated with control serum [379]. }\end{array}$ \\
\hline
\end{tabular}

Table 2: Summary of the role of different innate and adaptive immune cells in atherosclerosis. 


\begin{tabular}{|c|c|c|}
\hline Cell Subset & Suggested Role & Description \\
\hline TH1 cells & Pro-atherogenic & $\begin{array}{l}\text { Genetic deficiency in IFN- } \gamma \text { (TH1 signature cytokine) or its } \\
\text { receptor in Apoe- } /- \text { mice reduced lesion formation and } \\
\text { enhanced plaque stability }[127,134] \text {. } \\
\text { Mice lacking IL-12 or IL-18, TH1 promoting cytokines, show } \\
\text { reduced atherosclerosis development, whereas, injections of } \\
\text { IL-12 or IL-18 promote lesion formation }[136,137,138,139] \\
\text { Targeted deletion of Tbx } 21 \text {, the gene encoding TH1- } \\
\text { differentiating transcription factor T-bet, leads to reduces } \\
\text { lesion development in } L d l r^{--} \text {mice [141]. }\end{array}$ \\
\hline TH2 cells & Controversial & $\begin{array}{l}\text { IL-5 production is necessary for the production of protective } \\
\text { antibodies against oxLDL and IL-13 has profibrotic anti- } \\
\text { inflammatory properties [133]. } \\
\text { IL-4 production can induce proatherogenic effects [142]. }\end{array}$ \\
\hline TH17 cells & Controversial & $\begin{array}{l}\text { Apoe and Fcy chain deficient mice had less atherosclerosis } \\
\text { with reduced TH17 numbers [144]. } \\
\text { Blocking IL-17 with polyclonal anti-IL-17-neutralizing } \\
\text { antibodies raised in rats or goats resulted in significant reduction, } \\
\text { whereas rIL-17 treatment augmented atherosclerosis in Apoe- } \\
\text { mice. } \\
\text { IL-17 production was increased and atherosclerosis } \\
\text { markedly reduced Ldlr- }- \text { mice with suppressor of cytokine } \\
\text { signaling (SOCS) } 3 \text { deletion in T cells, which specifically } \\
\text { promotes T-cell polarization toward Th17 [145]. }\end{array}$ \\
\hline Treg cells & Athero-protective & $\begin{array}{l}\text { Genetic inactivation or blockade of TGF- } \beta \text { or IL- } 10 \text { by } \\
\text { neutralizing antibody accelerated lesion development [151, } \\
\text { 152, 153]. } \\
\text { Depletion of Treg cells in mice models with deletion of } \\
\text { CD80/CD86, CD28, ICOS or after treatment with CD } 25 \\
\text { neutralizing antibodies aggravated atherosclerosis }[154,155] \text {. }\end{array}$ \\
\hline CD8+ T cells & Pro-atherogenic & $\begin{array}{l}\text { CD8+ T cells stimulated by injection of an agonist to the } \\
\text { TNF- } \alpha \text { like surface protein CD137 or activated to an artificial } \\
\text { antigen expressed by SMCs increase atherosclerosis in } \text { Apoe }^{-1-} \\
\text { mice }[158,159] \text {. }\end{array}$ \\
\hline NKT cells & Pro-atherogenic & $\begin{array}{l}\text { Both chronic deficiency }\left(\mathrm{CD} 1 \mathrm{~d}^{--}\right) \text {and acute activation of } \\
\text { NKT cells in Apoe-/- or Ldlr-/- mice confirmed their } \\
\text { proatherogenic effects [165]. } \\
\text { Adoptive transfer of splenocytes from NKT enriched } \\
\text { Va14Ja18 TCR transgenic mice into } \mathrm{RAG}^{-/} \text {Apoe }{ }^{-/-} \text {mice } \\
\text { increased lesion size enormously compared to mice which } \\
\text { received NKT cell deficient splenocytes [168]. }\end{array}$ \\
\hline B cells & $\begin{array}{l}\text { B1: Anti-atherogenic } \\
\text { B2: Pro-atherogenic }\end{array}$ & $\begin{array}{l}\text { Transfer of B cell-deficient for serum IgM bone marrow into } \\
L d l r-/ \text { - exacerbated atherosclerosis [172]. } \\
\text { Depleting mature B cells with anti-CD20 antibody prevented } \\
\text { atherosclerosis development [146]. } \\
\text { Adoptive transfer of B-2 cell subset Apoe-/- Rag2-/- yc-/- } \\
\text { mice aggravated atherosclerosis [173]. }\end{array}$ \\
\hline
\end{tabular}

Table 2: Summary of the role of different innate and adaptive immune cells in atherosclerosis. 


$$
\text { -CHAPTER 2- }
$$

Natural Killer Cells 


\section{A. What Natutal Killer Cells are?}

It was only in the early 70's that Natural Killer (NK) cells were accidently discovered when research on the cytotoxic effects of $\mathrm{T}$ cells against target cells produced an unexplained background noise or "natural cytotoxicity" in non-immunized mice. Then, these cells were coined the name "Natural Killer" after their ability to sponateously kill target cells without prior sensitization. Originally, NK cells were described as cytotoxic effectors of innate immune system [174], [175]. Later, they were recognized as large granular lymphocytes widely distributed throughout the body in both lymphoid (BM, spleen, thymus, lymphnode) and non-lymphoid (uterus, liver, lungs) organs [176]. Then, they were distinguished from B and $\mathrm{T}$ cells and were referred to as "null lymphocytes"due to their lack of B and T cells' characteristic cell surface antigens [177], [178].

NK cells belong to the innate arm of the immune system whose cellular actors also include granulocytes, macrophages, and mast cells [178]. They serve to protect the host against infectious diseases, rapidly secreting perforin and granzymes to lyse infected cells, and proinflammatory cytokines such as IFN- $\gamma$ and TNF- $\alpha$ to alert additional host defenses [179]. However, due to the recent evidences on $\mathrm{NK}$ cell biological functions, the traditional classification of NK cells in innate immunity is currently discussed. NK cells have been lately described to possess some features of adaptive immunity including specific antigen recognition, clonal proliferation, and long-lived memory similar to B and T cells [180], [181]. The combination of innate and adaptive characteristics places NK cells at the boundary between these two immune compartments.

NK cells are educated during their development, thanks to the arsenal of surface receptors they express [182]. Therefore, they can recognize the loss of major histocompatibility complex (MHC) I on potential target cells in order to specifically recognize and eliminate target cells (immunosurveillance), while sparing healthy self-cells (immunotolerance).

The importance of NK cells in humans is supported by the higher risk of patients with NK cell deficiency to suffer from severe systemic and life threatening infections, including human cytomegalovirus (HCMV) [183]. Intriguingly, high activity of peripheral blood NK cells is associated with a $10 \%$ lower incidence of tumors for men and $4 \%$ for women, and their infiltration of certain tumor tissues is an indicator of better prognosis [184]. 


\section{B. Origin, Development, and Maturation of NK cells}

\section{Generation of NK cell progenitors from HSCs}

Based on the survival of adoptively transferred NK cells, the half-life of mature NK cells in the periphery is limited to about seven to ten days. Hence, NK cell renewal has to be continuous to maintain homeostasis.

Since NK cells are distinct from other lymphocytes and characterized by the absence of antigen receptors (Ig and TCR in B and T lymphocytes respectively), their development is unique such that it does not require events that lead to antigen receptor gene rearrangement [185].

BM is considered the primary site for generation of NK cells in adult (Colucci et al., 2003), although it has been suggested recently that at least part of extramedullary NK cells develop/mature in liver, spleen, and secondary lymphoid tissue [186], [187], [188]. However, extramedullary "NK poiesis" occurs under certain conditions and the NK cells produced are unique; they express the $\alpha$ chain of IL-7 receptor, which makes $<5 \%$ of total NK cells [189]. Selective BM ablation studies in mice, with estrogen (17-beta-estradiol) or bone-seeking isotopes ( $89 \mathrm{Sr}$ ), provided the first evidence that complete NK functional and phenotypic maturation requires an intact BM microenvironment [190], [191], [192].

In addition, normal development necessitates interaction with stromal elements, such as interactions between membrane lymphotoxin- $\alpha$ (LT- $\alpha$ ) expressing NK cell precursors and LT- $\alpha$-responsive stromal cells [193], [194], [185].

It is generally accepted that NK cells derive from CD34+ hematopoietic stem cells (HSCs) and its development occurs in several stages. The earliest step involves commitment of HSC to common lymphoid progenitor (CLP) that gives rise to B and T cells [195]. This step seems to be dependent on zinc-finger transcription factor Ikaros and PU.1 as deficiency in either transcription factors results in severe defects in the development of all lymphoid cells, while erythroid and myeloid lineage are less affected. The next step involves differentiation of CLP to bipotential T/NK progenitor (T/NKP), which can give rise to $\mathrm{T}$ and/or $\mathrm{NK}$ and not to other lineages. Subsequently, unipotent NK cell progenitors (NKP) destined to become mature NK cells are likely to be generated via T/NKP [196], [197]. This transition step from T/NKP to NKP is marked by the acquisition of IL-2/IL-15R $\beta$ subunit (CD122), which in turn renders NKP responsive to IL-15. Deficiency in IL-15, IL-15R $\alpha$, or IL-2R $\beta$, which forms part of IL15R complex, does affect NK cell development and produce a relatively selective NK cell deficiency [198], [199], [200]. 
Consistent with the essential role of IL-15 and its receptor, this transition step is also regulated by the transcriptional activity of the specific factors Jak3, STAT5a/b, and interferon regulatory factor 1 (IRF-1), which are important components of IL-15R signaling pathway. In addition, Ets1 transcription factor and Id2 DNA-binding protein are critical at this stage as deficiency in these transcription factors prevent development of NK cells but do not affect the development of T and B cells [201], [202], [185].

\section{Developmental Stages from NKP to mature NK cells}

Once committed to NK cell lineage, NKP must acquire the phenotypic and functional qualities that characterize mature peripheral NK cells [178]. Hence, during their maturation, NK cells change the expression of several cell surface markers, and gradually gain functionality and tolerance to self [188].

Whatever is the site of development and maturation, several stages can be distinguished in vivo. NK cell differentiation is initiated at a precursor stage I defined by the cell surface expression of CD122 and the lack of lineage (lin) markers [203]. Subsequently, at stage II, the cells acquire NK1.1 and integrin $\alpha \mathrm{v}$. They also express CD94/NKG2 and NKG2D. Stage III is defined by $\mathrm{NK}$ cell expression of c-kit (the stem cell factor receptor CD117) and Ly49 receptors. During stage IV, NK cells express CD16, CD49b1 integrin DX5 and proliferate significantly in the bone marrow. Thereafter, at stage V, as NK cells acquire high expression of Mac-1 (CD11b b2 integrin) and CD43, further proliferation markedly decreases unless challenged by viruses and pathogens [203], [204], [185].

On fully mature NK cells, c-kit and CD27 expression are lost whereas KLRG-1 and CD43 are expressed (Figure 8).

It is worth mentioning that Narni-Mancinelli (2011) has proposed recently a revised model of NK cell differentiation where the induction of NKp46 expression defines stage III of NK cell differentiation [205] (Table 3).

Importantly, during the perceived final stage of maturation, NK cells migrate to the periphery and acquire their full effector function producing high levels of IFN-y and exerting high levels of cytotoxicity [206], [207]. 


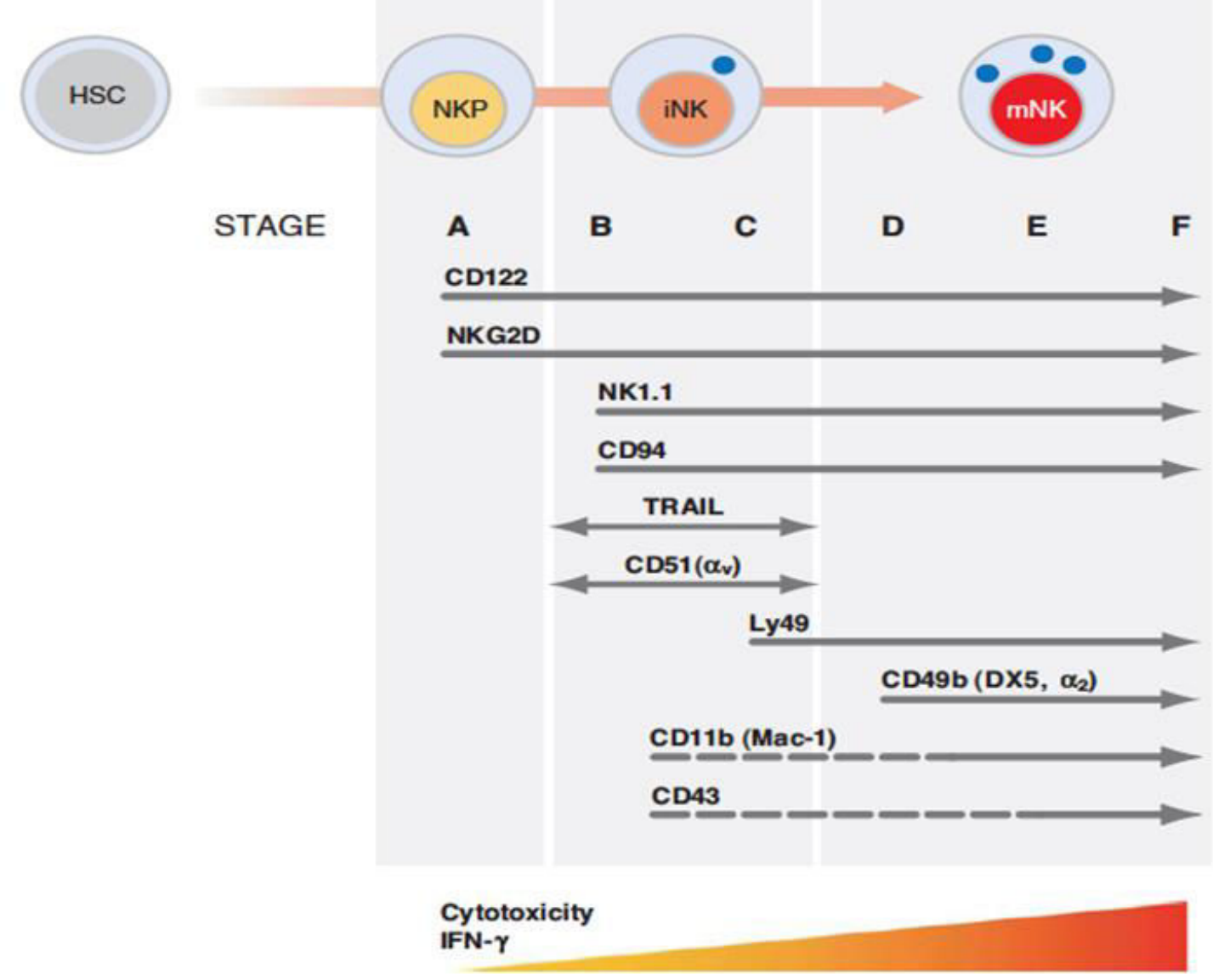

Figure 8: Phenotypic markers of developing NK cells in the mouse. NK cell transition from one developmental stage to another is marked by expression of cell surface receptors. CD122 and NKG2D are expressed by NKP then NK1.1 and CD94 by immature NK cells. Ly49 and DX5 receptors are relatively late markers of NK cell differentiation expressed by mature NK cells while CD11b and CD43 expression increase as NK cells differentiate. (adapted from: Di santo, Annu. Rev. Immunol., 2006)

Hayakawa and Smith (2006) have subdivided mature NK cells in the periphery into functional subsets on the basis of CD27 receptor expression. Subsequently, in 2010, Choisson suggested a 4-stage linear maturation model: $\mathrm{CD} 11 \mathrm{~b}^{\text {low }} \mathrm{CD} 27^{\text {low }} \rightarrow \mathrm{CD} 11 \mathrm{~b}^{\text {low }} \mathrm{CD} 27^{\text {high }} \rightarrow$ $\mathrm{CD} 11 \mathrm{~b}^{\text {high }} \mathrm{CD} 27^{\text {high }} \rightarrow \mathrm{CD} 11 \mathrm{~b}^{\text {high }} \mathrm{CD} 27^{\text {low }}$, which parallels the progressive acquisition of $\mathrm{NK}$ effector functions [208]. While CD27 ${ }^{\text {high }}$ cells possess a lower threshold to be activated, $\mathrm{CD} 27^{\text {low }}$ cells activation is tightly regulated [207].

The terminally differentiated $\mathrm{CD} 11 \mathrm{~b}^{\text {high }} \mathrm{CD} 27^{\text {low }} \mathrm{NK}$ subset in mice likely corresponds to CD56 ${ }^{\text {dim }} \mathrm{NK}$ subset in humans, suggesting that CD27 is regulated similarly during mouse and human NK cell differentiation [206]. 
In human, NK cells can be divided into two subpopulations based on the expression levels of CD56, CD56 ${ }^{\text {bright }}$ and CD56 $6^{\mathrm{dim}}$. CD56 $6^{\text {bright }} \mathrm{NK}$ cells are found mostly in lymphoid organs. CD56 ${ }^{\text {bright }} \mathrm{NK}$ cells are poorly cytolytic, more prone to produce cytokines, and proliferate vigorously after interaction with activated dendritic cells. By contrast, CD56 ${ }^{\mathrm{dim}} \mathrm{NK}$ cells, which are predominant in peripheral blood, can be both cytolytic and cytokine producing upon target recognition. It has long been proposed that CD56 $6^{\text {bright }}$ cells are precursors of CD56 ${ }^{\text {dim }}$ cells [209], [210], [208].

\begin{tabular}{|lcccccc|}
\hline & Stage 1 & Stage 2 & Stage 3 & Stage 4 & Stage 5 & Stage 6 \\
\hline CD122 & + & + & + & + & + & + \\
NK1.1 & - & + & + & + & + & + \\
NKp46 & - & - & + & + & + & + \\
CD16 & Low & Low & Low & + & + & + \\
CD11b & Low & Low & Low & Low & + & + \\
CD27 & - & Low & + & + & + +- & - \\
NKG2D & Low & Low & Low & + & + & + \\
DX5 & - & - & - & + & + & + \\
C-Kit & - & - & - & + & + - & + \\
Ly49 & - & - & - & + & + & + \\
CD94 & Low & + & + & + & + & + \\
CD43 & - & - & - & - & Low & + \\
KLRG1 & - & - & - & - & Low & + \\
\hline
\end{tabular}

Table 3: NK cell maturation. A revised model of NK cell maturation is based on the sequential expression of CD122, NK1.1, NKp46, CD16, and CD11b at NK cell surface. (adapted from: Narni-mancinelli, PNAS, 2011)

It is generally assumed that NK cell development is likely to be similar in human and mice. However, since these developmental stages are phenotypically defined by the expression of surface receptors, it is difficult to translate the developmental stages in mice to human [185]. Some differences have been described. For example, IL-12 deficient mice have normal NK cell number, while its presence is important for human NK cell development [211]. The same applies to IFN- $\gamma$ and IL-18, which are indispensable for human NK cell development, but their deficiency in mice does not affect NK cell number [212]. This difference between mice and human could be attributed to either in vitro/in vivo or species effects. 


\section{NK cell trafficking and tissue distribution}

Mature NK cells, which exit the BM, will eventually home to blood, spleen, liver, as well as uterus. Consistent with their role in immune surveillance, NK cells are widely distributed in lymphoid and non-lymphoid organs. In mice, the percentage of NK cells is higher in nonlymphoid than in lymphoid organs and the order for frequency is lung $>$ liver $>$ peripheral blood $>$ spleen $>$ BM $>$ LN $>$ thymus; the largest number of NK cells can be found in spleen of about 2-3 million [213].

NK cells migrate down a chemokine gradient to their respective niche. Firstly, it has been shown that $\mathrm{NK}$ cell maturation correlated with acquisition of $\mathrm{S}_{1} \mathrm{P}_{5}$, one of the five sphingosine-phosphate G-protein-coupled receptors. The extent of NK cell accumulation in

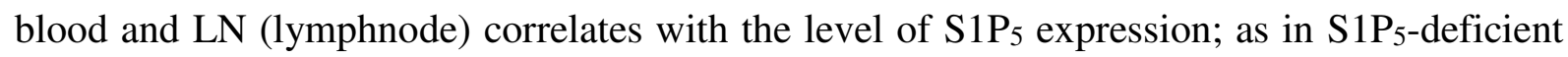
mice, NK cells accumulate in the BM and LNs, and are depleted from blood, spleen, and lung

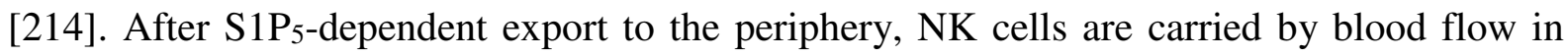
S1P5-independent manner.

Four types of cell surface receptors are involved in NK cell trafficking in mouse: the chemokine receptors $\mathrm{CCR} 2, \mathrm{CCR} 5, \mathrm{CXCR} 3$, and $\mathrm{CX}_{3} \mathrm{CR} 1$, which regulate mouse $\mathrm{NK}$ cell recruitment following inflammatory stimulus [213]. It has been observed that during inflammation, NK cell counts increase in liver and decrease in spleen and BM, suggesting that these organs serve as a reservoir for NK cells in case of inflammation [215]. While CXCR3 is partly required for mobilization of spleen NK cells, CCR1 is required for their accumulation in the liver. CCR2 chemokine receptor is also necessary for NK cell migration to the liver in response to CCL2 during mouse cytomegalovirus (MCMV) infection [213].

In addition, NK cells are preferentially recruited to the T-cell zone of LN near high endothelial venules (HEV) under inflammatory conditions. NK cell entry to LNs from blood is dependent on CD62L (L-selectin) [216]. Knowing that NK cells are normally undetectable in LN at steady state. Their massive recruitment upon inflammation, which is required for TH1 cell polarization of naïve T cells activated within LN, is mediated by CXCR3 ligands that are expressed on the surface of inflammatory HEVs [217], [213]. This highlights the importance of NK cells in shaping adaptive immunity. 


\section{NK cell Receptors and Ligands}

As effectors of innate immunity, NK cells rely on a broad array of inhibitory and activating receptors, as well as adhesion molecules to distinguish self from non-self or "altered self". The integration of signals received through these receptors governs the dynamic equilibrium of NK cell activation and dictates whether or not NK cells are to be activated [218].

These receptors are completely encoded in the genome, rather than being generated by somatic recombination, like T- and B-cell receptors [219].

Inhibitory receptors signal through intracellular immunoreceptor tyrosine-based inhibitory motifs (ITIMs), located in the receptor's cytoplasmic domain. Following ligation, these ITIMs become phosphorylated at tyrosine residues, allowing them to recruit tyrosine phosphatases (SHP1 or 2) and thus inhibit NK effector functions [220], [219].

In contrast, activating receptors, which are mostly transmembrane receptors, signal by interacting with transmembrane adaptor molecules, including DAP12, FcR $\gamma$ and CD3 $\xi$, which contain immunoreceptor tyrosine-based activating motifs (ITAMs). After phosphorylation of a tyrosine residue in the tail, the Src homology 2 domain containing kinases (Syk or ZAP70) are recruited, leading to a signal cascade, which results in degranulation and transcription of cytokine and chemokine genes [220].

A third signaling pathway employed by NK cells is the stimulation of the 2B4 receptor, which contains an immunoreceptor tyrosine-based switch motif that can recruit Src homology 2 domain containing adapter proteins SAP (SLAM-associated protein) or ERT (EAT2-related transducer). While NK cell function is inhibited after ERT recruitment, it is activated after recruitment of SAP [221].

A comprehensive overview of mouse and human NK cell receptors, well presented by Vivier and his colleagues [181], is displayed in Figure 9.

Receptor family more easily defines the recognition receptors on NK cells than functional categories of inhibitory and activating. These fall into two main structural classes: the immunoglobulin superfamily and C-type lectin-like family.

Therefore, each receptor will be discussed with respect to structure, proposed function, and regulation [221], [219]. 
Activating receptors

Receptors
NKp46
CD16
h NKp30
h NKp44
h NKp80
m NKR-P1C
NKG2D
m NKG2D-S
h KIR-S
m Act. Ly49
CD94/NKG2C
CRACC
Ly9
CD84
NTBA
2B4

Adaptors

$\mathrm{CD} 3 \zeta, \mathrm{FcR} \gamma$

$\mathrm{CD} 3 \zeta$, FcR $\gamma$

$\mathrm{CD} 3 \zeta, \mathrm{FcR} \gamma$

DAP12

-

FCR $\gamma$

DAP10

DAP12

DAP12

DAP12, DAP10

Inhlbitory receptors

DAP12

SAP, EAT2

SAP

SAP, EAT2

SAP

SAP, EAT2,

ERT

Cytokine receptors

IL-1R

IL-2R

h KIR-L

h LILRB1

CD94/NKG2A

$\mathrm{m}$ Inh. Ly49

m NKR-P1B

m NKR-P1D

Chemotactic receptors

KLRG-1

TIGIT

CEACAM-1

CCR2

CCR5

1 CXCR1

CXCR3

CXCR4

CXCR6

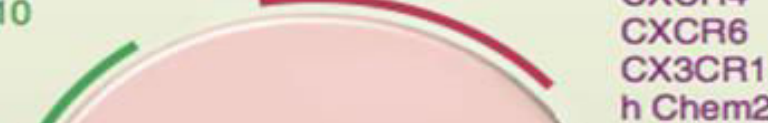

h Chem23R

S1P5

IL-12R

IL-15R

IL-18R

IL-21R

IFNAR

CD2

DNAM-1

$\beta 1$ integrins

$\beta 2$ integrins

Figure 9: NK Cell Receptors. NK cells express various cell surface activating (green) and inhibitory (red) receptors tuning their activity, in addition to adhesion (blue), cytokine (black), and chemotactic receptors (purple). Unless indicated (h, human; $\mathrm{m}$, mouse), receptors are conserved in both species. (adapted from: Vivier et al, Sicience, 2011)

\section{Immunoglobulin Superfamily}

\section{a) Killer immunoglobulin receptors (KIR) Family}

Human KIRs have evolved from Ig-superfamily and consist of type 1 transmembrane glycoproteins with two (KIR2D) or three (KIR3D) extracellular Ig-like domains. In relation to the function performed, the intracytoplasmic domains of KIRs can feature either a short (activating KIRs) or a long (inhibitory KIRs) cytoplasmic tail, named "S" or "L", respectively [221],[222]. The long tail generates an inhibitory signal resulting from the presence of ITIM 
motif in its cytoplasmic domain, whereas the short tail generates an activating signal due to their association with adaptor proteins bearing ITAMs [223]. The KIR family, located on chromosome 19p13.4, includes 12 members of which 6 receptors signal an inhibitory response and 6 signal an activating response following their binding to identical MHC I alleles. KIR receptors specifically recognize polymorphic epitopes on Human leukocyte antigen classes HLA-A, -B and -C proteins, among which HLA-C seems to have evolved as superior and more specialized ligand involved in regulation of human NK cells. Subsequently, it can provide either protection from or induction of target cell lysis and cytokine production. The expression of KIRs on different NK cells within one individual can vary with respect to allelic variants and levels of expression. Hence, each individual has different populations of NK cells that express an assortment of KIRs [223], [221].

The binding of inhibitory KIRs to MHC I ligands expressed by target cells ensures "self tolerance" and results in suppression of cytokine secretion and cytotoxicity. Different studies suggested that activating KIRs might interact with HLA class I at a lower affinity than their inhibitory counterparts. However, activating KIRs, particularly KIR3DS1, may play a role in NK-mediated response against viral infections, protection against hepatits $\mathrm{C}$, and delayed progression to acquired immune deficiency syndrome (AIDS). In addition, KIRs interact with members of other families of activating receptors such as NKp46 (see below) to potentiate CCR7 acquisition by $\mathrm{CD} 56^{\mathrm{dim}}$ population, whereby it becomes able to migrate in response to chemokines [222].

\section{b) Leukocyte Ig-like inhibitory receptors}

The (LIR)/Ig-like transcript (ILT) family maps on human chromosome 19 and consists of at least 10 genes coding for proteins of Ig superfamily. LIR/ILT are expressed on the surface of a proportion of NK cells (23-70\%) as well as on subsets of T cells, monocytes, B cells and DCs [224]. Its inhibitory function is mediated by tyrosine phosphorylation of four ITIMs in its cytoplasmic tailby tyrosine phosphatase SHP-1. While KIRs show allele-specific recognition of class I molecules, LIR-1 and LIR-2 interact with a broad range of classical and non-classical class I MHC molecules [225]. Ligands of LIR/ILT include class I HLA-G protein, some alleles of HLA-A and -B, and the human cytomegalovirus UL18 gene product [226].

Generally, LIR-1 functions as an inhibitory receptor and its engagement on NK and T cells by class I MHC molecules protects target cells from lysis [227]. 


\section{c) CD16a (FcyRIIIa)}

CD16 (or Fc $\gamma$ RIIIa), low affinity receptor for $\mathrm{IgG}$, was the first activation antigen described on NK cells. CD16a, a 70-KDa glycoprotein, is expressed as a transmembrane protein on the majority of human peripheral blood NK cells and on mouse NK cells. It can be also found on peripheral blood neutrophils and subsets of T cells. In NK cells, CD16a is covalently associated with $\zeta(\mathrm{CD} 3 \zeta)$, a signal transducing subunit of TCR complex, which transduces NK signals via its cytoplasmic ITAMs [228], [229]. CD16a is implicated in antibody dependent cell mediated cytotoxicity (ADCC), where it binds to antibodies that are attached to infected cells or pathogens, stimulating phagocytosis or cytolysis of infected cells (Note: ADCC will be discussed later in this chapter). Accordingly, cross-linking of CD16a by mAb has also been shown to increase transcription of IL-2R (CD25), TNF $\alpha$, and IFN- $\gamma$ mRNA [229]. CD16a is the only receptor able to trigger NK cell cytotoxicity on its own [230].

\section{d) DNAM-1 (CD226)}

Adhesion molecules can act either independently, initiating intracellular signaling pathways that trigger activation and adhesiveness of NK cells, or in conjunction with activating receptors to optimize cellular contacts or NK cell effector functions.

DNAX accessory molecule (DNAM-1, CD226) is one such Ig superfamily adhesion molecule. In humans, DNAM-1 is normally expressed on NK cells, CD8 T cells, monocytes, and CD4 T cells. However, in mice, DNAM-1 expression varies on NK cells ranging between 25 to $50 \%$, while it is constitutively expressed on CD8 T cells and induced on CD4, NKT and $\gamma \delta \mathrm{T}$ cells upon activation [231], [232], [233]. CD155 (poliovirus receptor) and CD112 (nectin-2), known as DNAM-1 ligands, are described in different pathological conditions and evidently upregulated on tumors. Activation through DNAM-1 induces its binding to CD112 and CD155 and recruits the tyrosine kinase Fyn and the serine-threonine kinase PKC [234], [235], [236]. This phosphorylation has proven to promote DNAM-1 association with lymphocyte function-associated antigen-1 (LFA-1) suggesting that DNAM-1 functions are partly mediated by LFA-1 integrin, which permits stable interaction with target cells [234], [237].

DNAM-1 has been implicated in NK-mediated killing of tumor cells [238], [239], [240], elimination of HIV-infected CD4+ T cells and HCMV-infected DCs [241], [242]. In addition, a recent study by Smyth and his colleagues suggested that DNAM-1 expression marks an alternative program of NK cell maturation. DNAM-1 ${ }^{-}$NK cells differentiate from DNAM- ${ }^{+}$, which produce high levels of inflammatory cytokines, have enhanced IL-15 signalling, and 
proliferate vigorously [236].

\section{e) Natural Cytotoxicity Receptors (NCRs)}

In the late 1990's, the three NCRs: NKp30, NKp44, and NKp46 were identified and cloned. While NKp46 and NKp30 are expressed constitutively by freshly purified peripheral blood NK cells, NKp44 is only induced upon NK cell activation.

NCRs belong to the immunoglobulin superfamily (Figure 10). They comprise an extracellular ligand binding domain, a transmembrane, and a short cytosolic domain. Since they lack functional intracellular signalling machinery, they associate with appropriate adaptor proteins via charged residues in their transmembrane domain [184]. Crystallography characterization has revealed that they are structurally different [243]. However, they share common ability to strongly activate NK cell cytolytic activity [222].

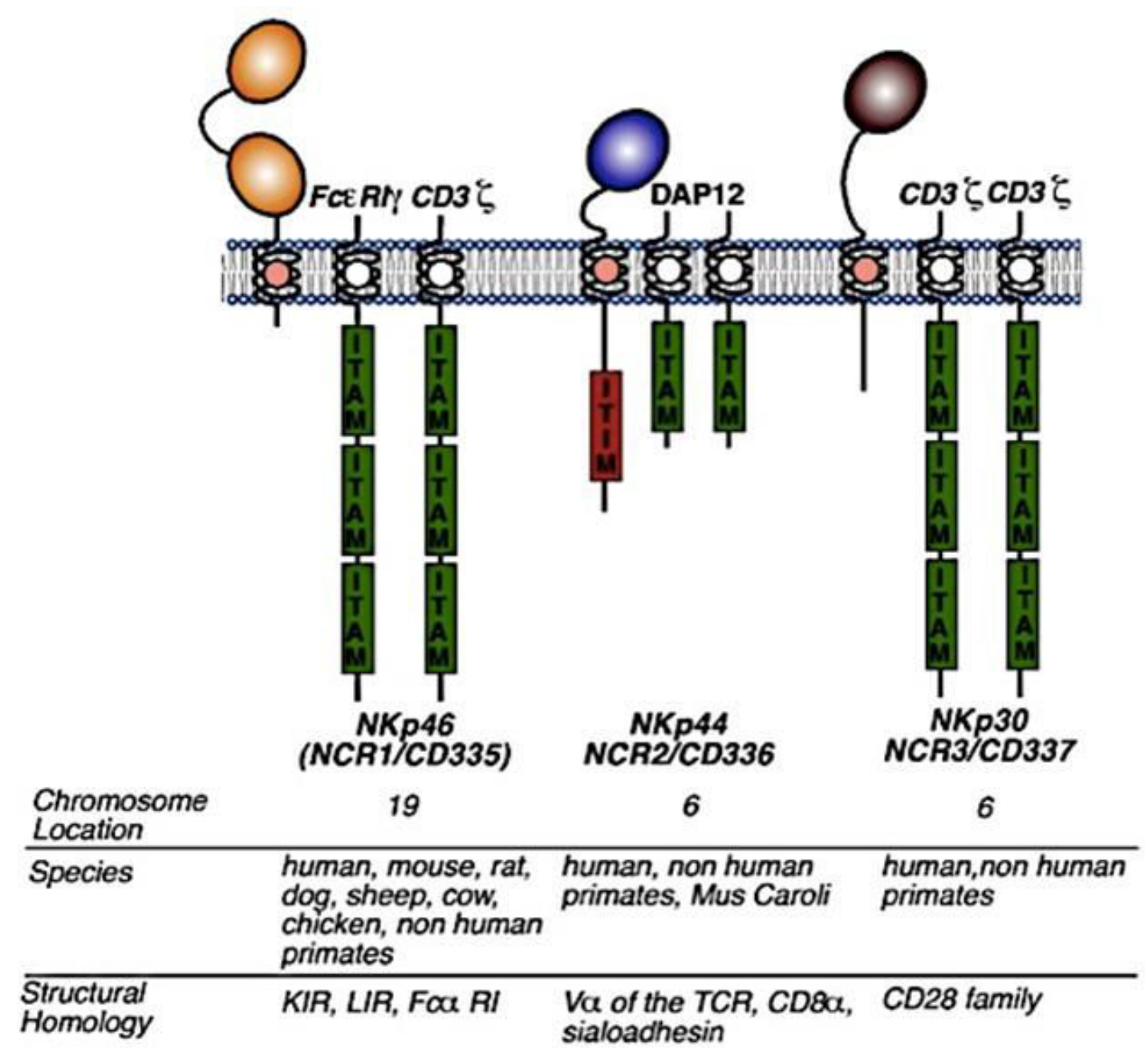

Figure 10: Structure of Natural Killer Cytotoxicity Receptors. Schematic depicting the molecular structure of NKp46, NKp44, and NKp30, the number of extracellular domains, the type of adaptor molecules to which they associate, their chromosomal location and species.

(adapted from: Hudespth et al, frontiers in immunology, 2013) 
NCRs have been suggested to be one of the main mechanisms through which NK cells kill tumor targets, whereby deletion of a single NCR reduces the ability of NK cells to lyse tumor targets in vivo [221]. Moreover, low expression of NCRs results in resistance of leukemia cells to NK cell cytotoxicity in patients with acute myeloid leukaemia (AML) [184].

NCR cellular ligands are still elusive, but may include those of viral origin or tumor expressed cellular ligands, as well as self-derived molecules. Viral ligands include hemagglutinin (HA) protein of influenza and vaccinia virus, Sendai and Newcastle proteins, and pp65, a component of HCMV virus recognized by NKp46, NKp44, and NKp30 respectively. Accordingly, in vitro and in vivo studies demonstrated that HIV-1 replication can induce the expression of ligands for NKp46, NKp44, and NKp30 on uninfected CD4 ${ }^{\text {pos }} \mathrm{T}$ cells, rendering these cells susceptible to NK cell-mediated killing [243].

Concerning self-molecules, human leukocyte antigen-B-associated transcript 3 (BAT3) has been identified as ligand for NKp30. The expression of this molecule on tumor cell surface triggers NK cell killing through production of TNF- $\alpha$ and IFN- $\gamma$. Intriguingly, BAT3 is also expressed by iDCs, which implicates NK cell in dendritic cell maturation by editing these antigen presenting cells.

As for tumor ligands, a common reported ligand for both NKp30 and NKp46 is the membrane associated heparan sulfate proteoglycan (HSPG) on various tumor cells. As for NKp44, it has been shown to interact with proliferating cell nuclear antigen (PCNA) expressed by tumor cells. In contrast to the effect of NKp46 and -30 binding to their ligands, NKp44 ligation results in inhibitory effect, which explains the unusual presence of inhibitory motif ITIM within NKp44 intracellular domain [243].

Although NCRs are expressed almost exclusively on NK cells, new studies reported their expression on minute subsets of T cells. NKp44 was expressed on in vitro expanded $\gamma \delta \mathrm{T}$ cell [244]. However, circulating $\gamma \delta \mathrm{T}$ cells are $\mathrm{NCR}^{\text {neg }}$ and NCRs are induced on the surface only through engagement of TCR in synergy with cytokine stimulation [243]. In addition, NKp44 expression is induced on a small subset of IFN- $\alpha$ producing plasmacytoid dendritic cells (pDCs) isolated from human tonsils in an IL-3 dependent manner [245]. Later on, NKp46 was found to be expressed on TCR $\alpha \beta$ intestinal intraepithelial lymphocytes (IEL) [246]. Moreover, NKp46 is expressed on IL-22 producing innate lymphoid cells (LTi) that can be found in human fetal mesenteric lymphnodes, tonsils, and the intestine, as well as murine cryopatches [247], [248], [249], [250], [243]. 


\section{C-type Lectin Superfamily}

\section{a) Ly49 Family Receptors}

Mice use the lectin-like receptors of the Ly49 family as an equivalent to the human KIR [251]. Although structurally distinct, they appear to be functionally similar to human KIRs in terms of MHC-I recognition. These receptors are encoded by the C-type lectin-like Ly49 multigene family that maps on chromosome 6 in a region termed NK gene complex (NKC). Then, they are expressed at the cell surface as transmembrane disulphide-bonded homodimeric type II transmembrane proteins [252]. The majority of these receptors are inhibitory such as Ly49A and Ly49C, and signal through ITIMs, although activating receptors such as Ly49D do exist and associate with DAP-12 molecule for signaling [221].

Ly49 expression is not restricted to NK cells but rather can be found on cells of both innate and adaptive immunity, such as CD8+ T cells, CD3+ cells, intestinal epithelial lymphocytes (IELs), NKT cells, uterine NK cells (uNK), and cells of myeloid lineage [251].

\section{b) NKR-P1 Receptors}

The NKR-P1 family has been of considerable interest due to its conservation in rats, mice, and human [253]. In mice, these receptors are either activating or inhibitory co-stimulatory receptors, for example NKR-P1B acts as inhibitory receptor whereas NKR-P1C acts as an autonomous activating structure. They are type II membrane glycoprotein receptors that belong to the C-type lectin family [221].

The first member of this family to be discovered, NKRP1C, is still known by its original name NK1.1 and is widely used as a marker for identifying and defining NK cells and NKT in C57BL/6 mouse strain [254]. NK1.1 associates with ITAM containing Fc $\gamma$ chain to deliver an activating signal although the biological relevance of this is still not clear [221].

NKR-P1 receptors do not recognize common MHC I-like molecules and physiologic ligands remain to be identified.

In human, only NKR-P1A (CD161) is expressed, which can be also detected on CD3+ T cells, CD4+ T cells, and NKT cells. Although no ligands for this receptor have been identified, engagement of CD161 on cultured NK cells can result in inhibition of cytotoxicity [255].

\section{c) CD94-NKG2 Heterodimer Receptors:}

CD94/NKG2 family of receptors has individual members with activating and inhibitory 
features due to some structural characteristics in their intracytoplasmic and transmembrane domain [256]. The inhibitory members are NKG2A and -2B and signal through ITIMs in their cytoplasmic tail while the activating members NKG2C, $-\mathrm{E}$, and $-\mathrm{H}$ associate with DAP-12 ITAM and signal via Syk and ZAP-70 [257].

The ligand for CD94/NKG2 receptors is the non-classical class I molecule HLA-E in humans and Qa-1 in mice. The affinity of HLA-E is ten fold higher for the inhibitory human NKG2A than the activating $\mathrm{NKG} 2 \mathrm{C}$ receptor, which is also true for other paired inhibitory and activating ligands [258], [259], [260].

\section{d) NKG2D Receptor}

Although initially identified on NK cells, NKG2D (natural killer group 2, member D) was later found to be expressed by CD8 T cells (activated CD8 T cells), NKT cells, and subsets of $\gamma \delta$ T cells [261], [262], [263], [264].

Only distantly related to NKG2 family, NKG2D does not form a heterodimer with CD94, but is expressed as a homodimer and signals by recruiting DAP-10 or DAP-12 molecules[221]. Two isoforms exist for NKG2D in mouse; the longer isoform (NKG2D-L), which can only recruit DAP-10 and a shorter isoform (NKG2D-S), which can recruit either DAP-10 or DAP12 [221]. Notably, human NK cells only express the long isoform, which associates with DAP-10 to induce both cytotoxic and cytokine-mediated response.

Activation of NK cells through NKG2D overrides the inhibitory signal delivered by MHC I molecules and can even co-stimulate TCR-dependent activation of CD8 T cells [262].

NKG2D expression and signaling can be regulated by cytokines and tumor derived factors. While IL-2, IL-7, IL-12, IL-15, and type I interferons (IFNs) increase NKG2D transcripts, IL21 , IFN- $\gamma$, and TGF- $\beta$ have the opposite effect [264], [265]. For example, tumor cells can evade NKG2D through secretion of transforming growth factor- $\beta 1$ (TGF- $\beta 1$ ) which leads to down-regulation of expression of NKG2D on NK cells [221].

NKG2D ligands, rarely expressed in normal tissues, are inducible in response to variety of stimuli, including genotoxic and oxidative stress, heat shock, differentiation factors, viral infections, and tumor transformation. They are structurally related to MHC I molecules. In human, these include class I chain- related molecules A and B (MICA and MICB) and retinoic acid early transcripts (RAET-1) known as UL-16 binding proteins (ULBP-1, -2, -3, 4, and -5) [266]. In mice, five different RAET1 isoforms, three different H60 isoforms (a, b, and c), and MULT-1 have been described [264].

Indeed, studies provided a role for NKG2D in enhancing tumor immune-surveillance against 
cancerous cells, as well as induction of CTL, Th1, and Th2 responses [221].

\section{Toll like Receptors (TLRs)}

Structurally, TLRs are type I transmembrane proteins characterized by the extracellular domains containing leucine-rich repeats that mediate recognition of pathogen associated molecular patterns (PAMPs), transmembrane domains, and the intracellular Toll-interleukin 1 (IL-1) receptor (TIR) domains [267].

Human NK cells express functional TLR3 and TLR9 that allow responses to microbial products such as dsRNA (virus derived) and CpG (of bacterial origin) [268].

TLR3 is an endosomal receptor that senses viral dsRNA, which leads to secretion of type I interferon (IFN) and other pro-inflammatory cytokines [269].

For signal transmission, TLR3 links Toll-IL-1 receptor domain containing adaptor molecule-1 (TICAM-1) or adaptor protein TIR domain-containing adapter inducing IFN- $\beta$ (TRIF) in the cytoplasmic domain (TIR). Subsequently, multiple signaling pathways are initiated through these adaptors, which induce activation of transcription factors: extracellular signal-regulated kinase (ERK), Janus kinase (JNK), and p38 members of mitogen activated protein kinase (MAPK) family, and nuclear factor $\kappa \mathrm{B}(\mathrm{NF}-\mathrm{kB})$ and interferon regulatory factor 3 (IRF-3) [269], [270], [271]. This results in the expression of genes encoding type I IFNs: IFN- $\alpha$ and IFN- $\beta$, proinflammatory cytokines, and various molecules involved in antigen presentation [272], [273].

Poly (I:C), a synthetic analogue of double-stranded RNA (dsRNA), mimics the effects of naturally occurring dsRNA and is used as an adjuvant [274]. Poly (I:C) has been extensively used to activate mouse NK cells in vivo and cultured human NK cells [273]. It can induce efficient NK responses either by direct activation of TLR3 on NK cells or via activation of accessory cells such as DCs and CD8 $\alpha$ DCs [269].

In addition to TLR3, poly (I:C) can also exert its effects through the cytosolic sensor, melanoma differentiation-associated protein-5 (MDA5) [269]. MDA5 transmits signals through the adaptor IPS1 and leads to activation of similar array of transcription factors induced by TLR3 [273]. 


\section{Receptors of chemokines}

IL-2, IL-15, and TGF- $\beta$ induce the upregulation of the expression of inflammatory chemokine receptors $\mathrm{CXCR} 3$ and $\mathrm{CCR} 2$ in NK cells so that NK cells respond chemotactically toward the ligands of these receptors. This is mostly evident at endothelial cell surface where higher upregulation of inflammatory cytokines is associated with engagement of adhesion molecules [275].

Relatively, few chemokines have been shown to enhance the cytolytic activity of NK cells. Chemokines such as CCL3, CXCL10, CCL2, and CCL5 consistently augment NK cell cytotoxicity towards NK-sensitive tumors in a manner comparable to that produced with optimal concentrations of IL-2 [276], [277], [278].

The mechanism by which chemokines augment NK cell lysis of target cells is either by facilitating the discharge of cytotoxic granules or by inducing the redistribution of adhesion molecules on NK cell surface[279], [280], [278].

\section{E. NK cell effector function}

When activating signals predominate over inhibitory signals, NK cells effector functions are initiated [281], [282].

NK cells employ various mechanisms to kill target cells. They can be cytolytic through granule exocytosis or death receptor engagement or can be directly activated to induce antibody dependent cell mediated cytotoxicity. Also, they can produce an array of cytokines (IFN- $\gamma$ and GM-CSF) and chemokines (such as CCL3, CCL4, and CCL5).

\section{Granule exocytosis pathway}

Perforin/granzyme-mediated apoptosis is the principal pathway used by NK cells to eliminate virus infected or transformed cells [283].

This mechanism is common with CTL such that killing of the target cell involves firm adhesion and formation of immunological synapse (IS). Cytotoxic granules are then polarized towards the IS whereby the lymphocytes release their content within the synaptic cleft leading to lysis of the target cell [284], [285], [286], [287].

The granules contain a number of proteins, including a pore-forming protein, termed perforin and a family of serine proteases, called granzymes [228], [288].

Perforin causes osmotic damage due to its binding of phosphorylcholine headgroups, polymerization, and subsequent pore formation in the lipid bilayer of the target cell [228]. 
Perforin is an important component of NK cell killing machinery, as perforin deficient mice have severely limited capacity to eliminate syngeneic MHC I-deficient tumors [289], [290], [219]. In humans, mutation in perforin ends up in familial haemophagocytic lymphohistiocytosis due to impaired NK cell cytotoxicity [291], clearly demonstrating the crucial role perforin has as an immune effector molecule.

Granzymes play a supplementary role in target cell killing, supported by in vitro and in vivo experimental evidence. They synergize with perforin to trigger an "internal disintegration pathway" in the target cell. NK cells express three granzymes, Granzyme (Grz) A, B, and M that can induce target cell apoptosis either by activation of cellular caspases or in a caspaseindependent pathway [228], [292].

While perforins function to deliver granzymes to the target cell, granzyme uptake can be sometimes perforin-independent and is performed by receptor-mediated endocytosis. Particularly, mannose-6 phosphate receptor can act as a receptor for GrzB uptake when it is overexpressed on the surface of certain cells [293].

Recently, Alter et al identified CD107a/LAMP-1 (lysosome associated membrane protein-1) as a marker of degranulating cytotoxic lymphocytes because its surface expression is induced upon degranulation.CD107a is a highly glycosylated lysosomal proteins lining the membrane of cytolytic granulesand facilitating their mobility after activation [294], [295]. It might also serve to protect NK cells from degranulation-associated damage as deficiency in CD107a resulted in increased NK cell apoptosis upon target cell-induced degranulation [287].

\section{Death receptor pathway}

Death receptors represent an alternative pathway of NK cell mediated-cytotoxicity. It relies on target cell expression of TNF receptor superfamily members.

The two main receptors used in apoptotic induction are Fas (CD95) and TNF-related apoptosis-inducing ligand (TRAIL). These contain a conserved intracytoplasmic "death domain", which by recruiting adaptor molecules activate caspase enzymatic cascade and ultimately apoptotic mechanisms [296], [297].

\section{Activation by- and production of cytokines}

NK cells constitutively express receptors for monocyte-derived cytokines (monokines) and produce critical cytokines such as IFN- $\gamma$ in response to monokine stimulation [298].

IL-2, IL-4, IL-7, IL-12, and IL-15 can induce human NK cells to proliferate. However, NK cell proliferation in response to IL-2 and IL-15 is about 10 fold greater than the proliferation 
to other mitogenic cytokines [278].

In addition, exposure to cytokines such as IL-2, IL-12, and IL-15 enables NK cells to lyse solid tumors that are resistant to lysis by unstimulated NK cells [299], [298], [300], [278]. In parallel, work from the Yokayama lab demonstrated that activation of NK cells with the cytokines IL-12 and IL-18 has long lasting effects on NK cell activity. Following adoptive transfer, IL-12/IL-18 activated NK cells were capable of more vigorous IFN- $\gamma$ responses for several weeks post-transfer [301].

Similarly, during viral infections, efficient antiviral NK cell activity appears to require type I IFN-responses. IL-12 is required for IFN- $\gamma$ production, type-I IFN for enhanced cytotoxicity, and IL-15 for proliferation and survival [302]. Within 1.5 days after infection, at the time of initiation of NK cell activation, type I IFNs are produced mainly by pDCs while IL-12 can be produced by monocytes/macrophages and all DC subsets [303]. IL-18 synergizes with IL-12, IL-15, or type I IFN to amplify NK cells effector functions during viral infections.

NK cells secrete a number of effector cytokines such as IFN- $\gamma$, TNF- $\alpha$, granulocytemacrophage colony-stimulating factor (GM-CSF), IL-5, IL-10, and IL-13. Through production of cytokines, it participates in regulation of myeloid hematopoiesis, activation of myeloid and monocytic cells, and possibly in regulation of humoral immune responses. NKcell production of TH1 (IFN- $\gamma$ ) and TH2 (IL-13 and IL-5) cytokines occur at distinct stages of NK cell differentiation. Mature NK cells loose their ability to produce type 2 cytokines and acquire the ability to produce IFN- $\gamma$ [304], [219].

Importantly, NK cells are the primary source of IFN- $\gamma$ during the early innate immune response, which is key to control of viruses [282].

IFN- $\gamma$ transcription begins early during NK cell development in the bonemarrow, increases during its maturation, and is rapidly induced by NK activation, followed by its translation and secretion [305].

In addition to its immediate inhibition of viral replication, IFN- $\gamma$ exerts immunostimulatory and immunomodulatory effects. It induces upregulation of MHC I and MHC II antigen presentation by increasing the expression of subunits of MHC I and II molecules. It also contributes to macrophage activation by increasing phagocytosis and priming the production of proinflammatory cytokines. Moreover, IFN- $\gamma$ controls differentiation of naïve CD4 T cells into TH-1 effectors, which mediate cellular immunity against viral and intracellular bacterial infections [306], [307].

However, IFN- $\gamma$ production is influenced by the cytokine repertoire of the inflammatory 
milieu. While cytokines produced by monocytes: IL-2, IL-12, IL-15, IL-18, and type I IFN enhance IFN- $\gamma$ production, TGF- $\beta$, IL-4, and IL-10 inhibits its expression by NK cells, thereby avoiding its pathogenic role in autoimmune and autoinflammatory diseases [308], [309]. Alternatively, NK cell derived IL-22 ensures inhibition of the growth of $M$. tuberculosis and NK-derived IL-10 may be required to prevent immune pathology during systemic infections [310], [311].

Classically, the interaction of NK cells with target cells leads to cytolysis and IFN- $\gamma$ production. Vivier and his colleagues suggested that although cytotoxicity and cytokine production are thought to involve common signaling pathways, the threshold for the reactivity of NK cells in those two types of responses seems to be different (Figure 11). In most cases, the fraction of NK cells that become cytolytic after contact with target cells (as judged by cell degranulation) is higher than the fraction of $\mathrm{NK}$ cells that produce IFN- $\gamma$ in the same conditions [282].

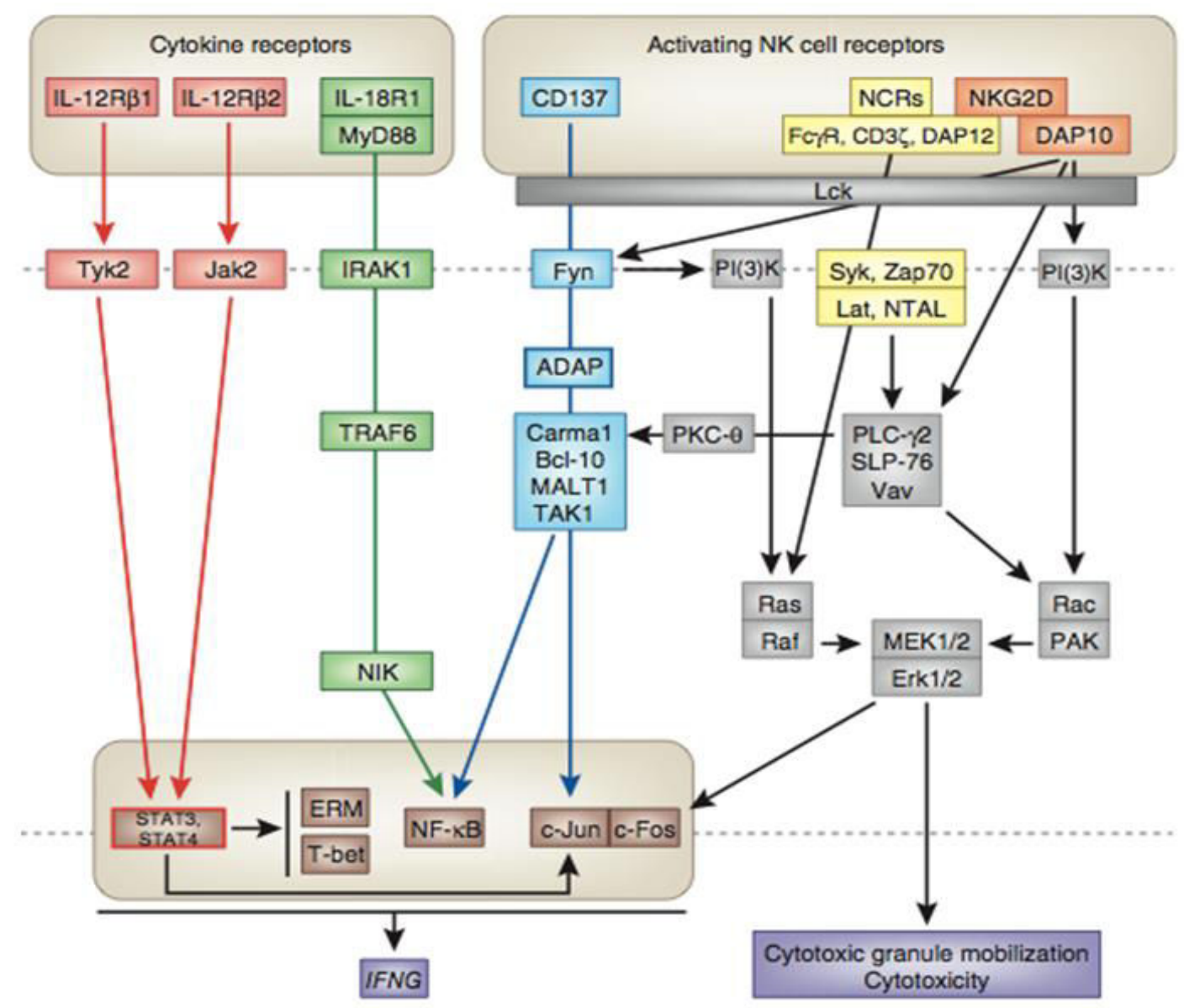

Figure 11: Signaling pathways leading to NK cell activation. Signaling pathways, initiated either through cytokine receptors or activating NK cell receptors, end up in IFN-g production or cytotoxic granules mobilization. (adapted from; Vivier et al, Nat. Rev. Immunology, 2013) 


\section{Antibody dependent cell mediated cytotoxicity}

NK cells express the low affinity IgG receptor CD16, which enables them to recognize and kill target cells opsonized with antibodies by antibody dependent cell-mediated cytotoxicity [312].

Antibody dependent NK-mediated tumor killing occurs through one of the pathways described above, either granule exocytosis or cytokine secretion. This mechanism is now highly exploited in mAb-based cancer immunotherapy [313].

\section{F. Regulation of NK cell activity}

\section{Missing Self Recognition by NK cells}

NK cell activation is controlled by multiple activating and inhibitory surface receptors [314]. Tumors or infected cells expressing low levels of surface MHC-I and overexpressing ligands for activating receptors will be killed by NK cells (Figure 12).

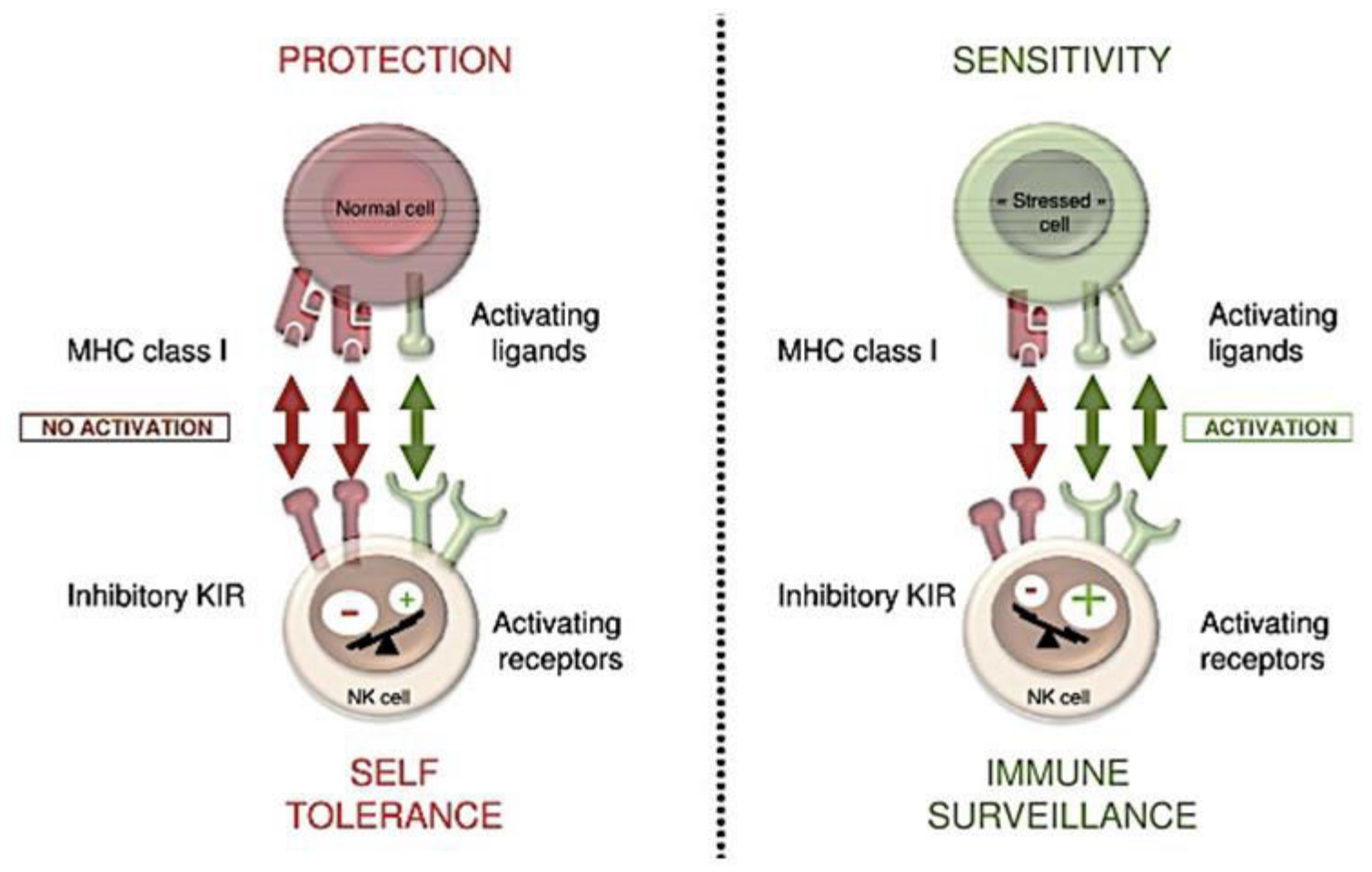

Figure 12: Balance between inhibitory and activating signals governs NK cell function. (adapted from; Vivier et al, medicine reports, 2011) 
Initial observations by Klas Karre and his colleagues revealed that NK cells kill cells lacking MHC-I expression while sparing MHC I sufficient cells. This was proposed after loss of selfMHC I expression by murine lymphoma cells triggered NK cell effector function [315]. Then, the recognition and lysis of MHC $\mathrm{I}^{\text {low }}$ target by NK cells was referred to as missing-self recognition. An underlying requirement for this "missing self hypothesis" model is that each NK cell expresses at least one MHC I-specific receptor, which enables it to detect presence/absence of self [316], [317].

\section{NK cell Education by MHC I molecules}

Since some NK cells lack inhibitory receptors that bind MHC I molecules of the host or express activating receptors that recognize self-ligands, they have the potential to be auto reactive, like B and T cells [181]. However, NK cells lacking self-MHC-I specific inhibitory receptors are not auto-reactive but rather have acquired a state of hypo-responsiveness to tumor cell and activating receptor triggering. Therefore, NK cells are held in check by a process known as education, whose ultimate effect is to have self-tolerant but yet useful NK population in terms of its ability to mediate "missing self recognition".

Currently no marker exists for educated NK cells and only functional assays can be used for the distinction between educated and non-educated cells. The overall functional consequence of NK education is such that the binding to self-MHC I molecules results in the ability of NK to respond to tumor cell, MHC I deficient cells, and antibody-mediated crosslinking of activating receptors in vitro. In vivo, this is represented by the ability of NK cells to reject hematopoietic BM grafts [318].

Several models have been proposed to describe various aspects of NK cell education (Figure 13). The first model: arming model or NK cell "licensing", coined by Yokayama and Kim in 2005 suggests that self-MHC I specific inhibitory receptors induce functional maturation (arming) of precursor cells. NK cells lacking self-MHC I specific inhibitory receptors persist as immature, hypo-responsive cells in terms of cytotoxicity and cytokine secretion following stimulation. In the second model, disarming model, NK cells that lack inhibitory receptors for self-MHC I could be disarmed; persistently stimulated and consequently induced to enter a state of hypo-responsiveness. This results in NK cell "anergy", similar to that found for T and B cells. Yet, they can only maintain their functionality if this chronic stimulation in counteracted by signals of inhibitory receptors. Hence, the difference between the two models is that the disarming model suggests that hypo-responsiveness is actively induced, whereas the arming model postulates that NK are inactive by default andfull functionality is achieved 
after interaction with MHC I-expressing cells [318], [319].
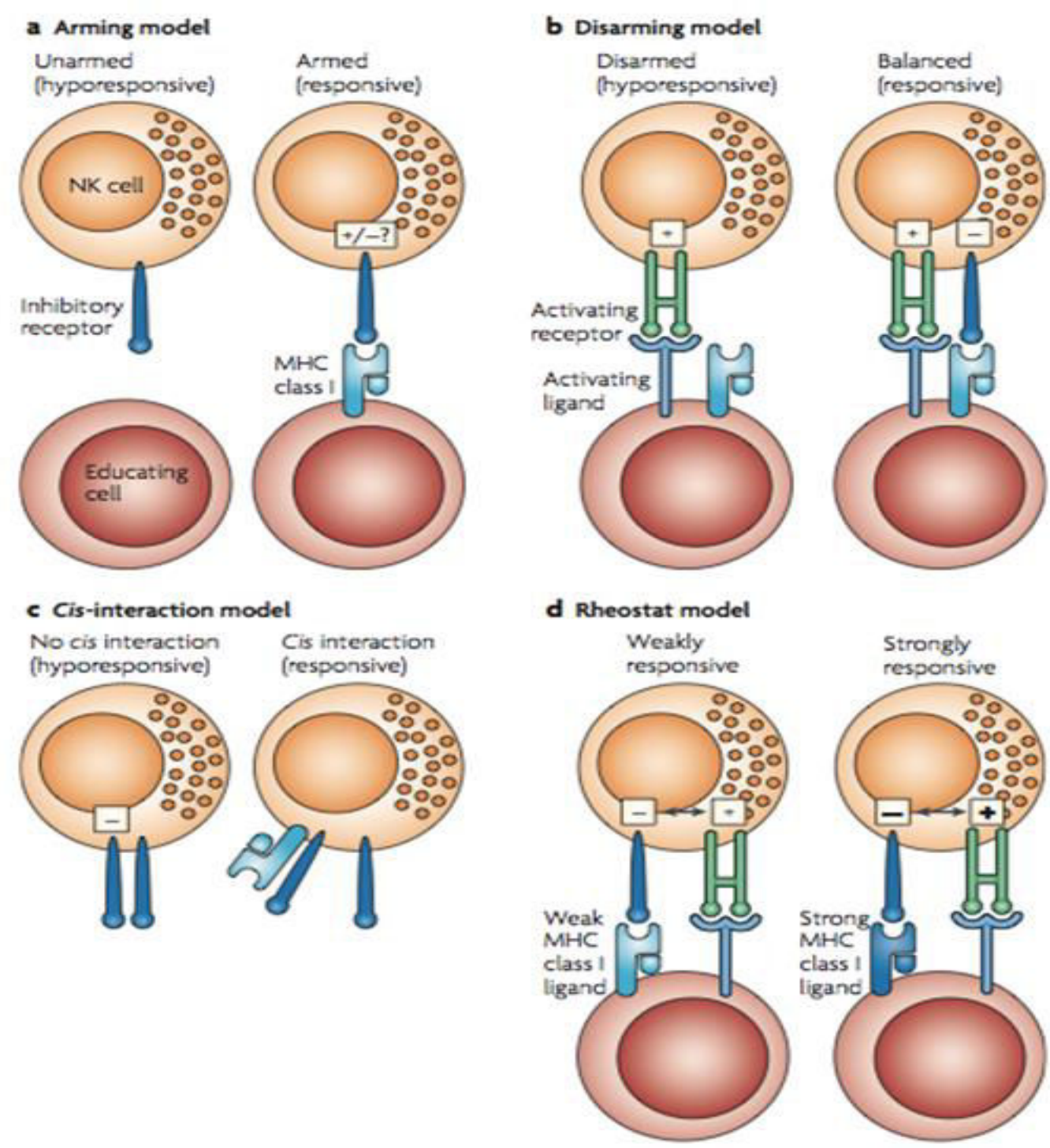

Figure 13: Models proposed for NK cell education. (adapted from: Hoglund et al, Nature Reviews in Immunology, 2010)

Up to this end, a third model "Rheostat model" has emerged to suggest that education is not an "all or nothing" decision but is more likely a quantitative process. The stronger the inhibitory interactions of an NK cell, the stronger it responds to activating receptor signals. This model supplements the arming or disarming models, such that NK cells can be either tuned up (arming) or tuned down (disarming) but in a quantitative manner depending on the frequency and strength of signal received by individual NK cells [317], [182], [320], [321]. Last, the fourth model emerged after observation that some inhibitory receptors, like Ly49 receptors, can transmit inhibitory signals as unengaged receptors in the absence of ligand 
interaction, by accumulating in the immunological synapse at the NK/target cell interface. Cis interaction between MHC I- and Ly49-receptors (Cis: on the same cell membrane) sequesters Ly49 receptors and prevents their relocation to the immunological synapse. As a result, inhibition via Ly49 receptor is reduced and NK cells become more responsive [318], [317], [322].

Recently, new studies suggested that NK cell tuning not only relies on signals from inhibitory receptors, but also on activating receptors such as NKG2D, KIR2DS1, or Ly49H, which induce NK hypo-reactivity in the chronic presence of their ligands[218], [323]. In addition, engagement of NKp46 activating receptor is also required to tune NK cell responsiveness. This was demonstrated in NKp46-deficient Noé mice. NK cells from these mice have lower activation threshold than WT cells, are hyper-responsive to several stimuli in vitro and exhibit higher resistance to viral infections in vivo. Genetic complementation studies with a human NKp46 transgene restored the reactivity of Noé NK cells to a level comparable to WT cells [324], [325].

Finally, it has been demonstrated that changes caused in educated and hypo-responsive (uneducated) cells are reversible. Uneducated NK cells gained functional competence after transfer to MHC I-sufficient environment, whereas previously educated cells were rendered hypo-responsive in MHC I-deficient environment [326], [327]. Altogether, these studies support the notion that NK tuning is very plastic and the threshold of NK activation is dependent on their adaptation to a given environment.

\section{DCs-mediated IL-15 trans-presentation}

Until recently, NK cells were thought to be poised and ready to kill target cells on contact. But the concept of "natural killer" is now challenged by multiple data sets indicating thatthe intensity and quality of NK cytotoxic and cytokine responses depend on the cytokine microenvironment and accessory cells.

In mice, resting splenic NK cells display relatively poor cytotoxic potential because of reduced expression of granzyme B and perforin, which are induced upon appropriate inflammatory context (cytokine stimulation or MCMV infection). Similarly, resting human peripheral blood NK cells harbor poor effector functions [218], [323], [328].

Hence, regulation of NK celleffector function and responsiveness also occur in mature circulating NK cells in response to cytokines and DC-mediated priming. Type I IFN, IL-12, IL-18, and IL-15 are potent activators of NK effector function [329], [330].

Lucas defined specific requirements for priming of NK cells and delineated a cascade of 
activation [331]. These include secretion of type I IFN by TLR+ cells, expression of type I IFN receptor by DCs, stimulation of IL-15R $\alpha$ and IL-15 expression by DCs, and transpresentation of IL-15 by DCs to NK cells in the LN (Figure 14). Priming induced cytotoxic activity of NK cells and their ability to produce IFN- $\gamma$ [331], [332].

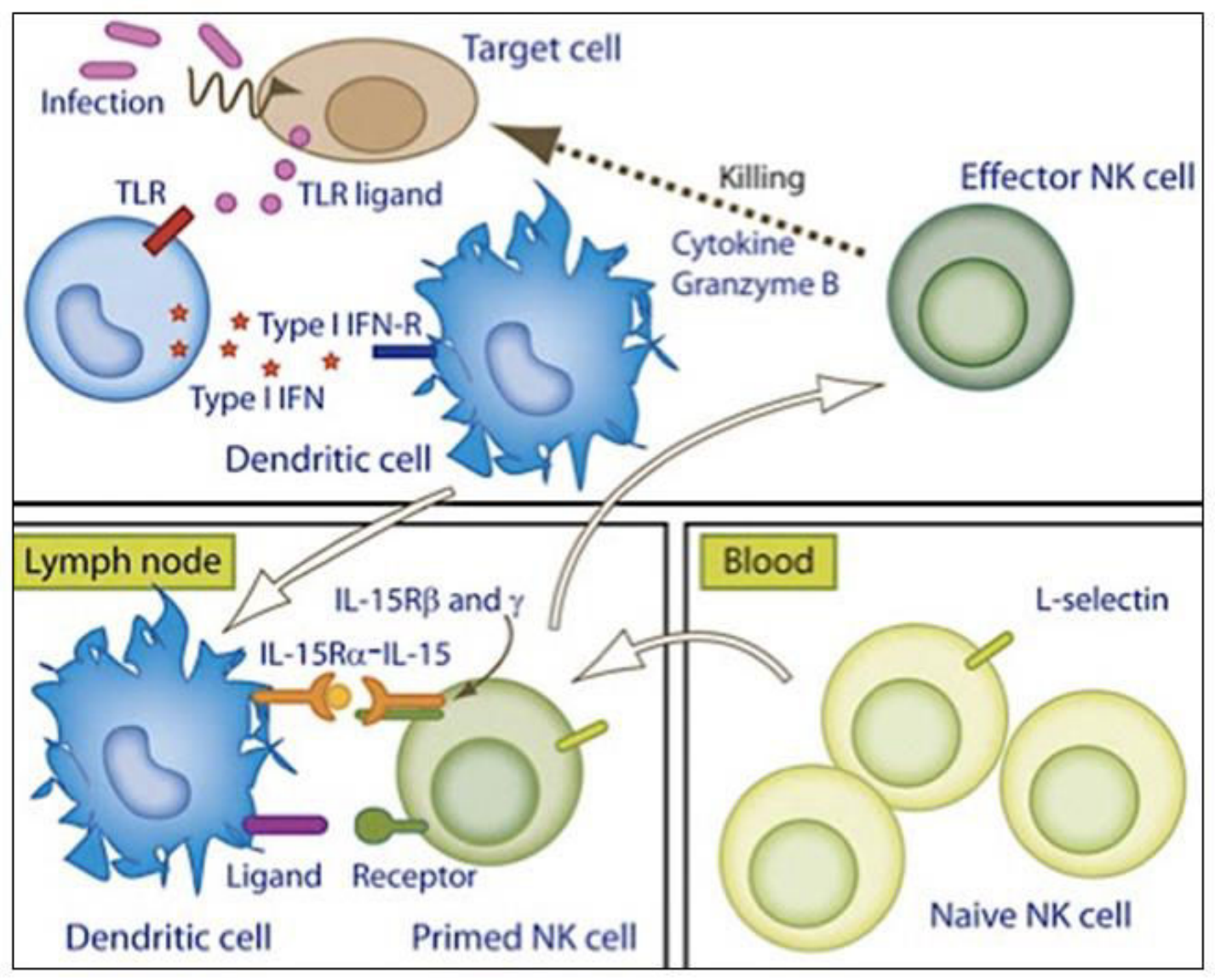

Figure 14: Priming NK cells in vivo. Infected cells secrete TLR ligands which stimulate TLR+ cells (DCs) to secrete Type I IFN. Type I IFN upregulates IL-15 receptor expression on DCs. DCs transpresent IL-15 and prime NK in the draining lymphnodes. (adapted from; Long, immunity reports, 2007)

In parallel, the TLR3 ligandpoly IC primes NK cells indirectly through presentation of IL-15 on activated DCs [331]. Maintenance of a pool of primed NK cells would be advantageous. Since priming is not antigen specific, primed NK cells would be poised to respond to tumor or virus infected cells even if these cells do not elicit type I IFN production for recruitment of NK cells to lymph nodes [332]. 


\section{NK-Treg cross talk}

A third layer of regulation of NK cell activity that relies on the adaptive immune system, Treg and effector CD8+ T cells, has been recently revealed [323].

IL-2 has long been used in vitro to stimulate NK cell proliferation and activation leading to generation of lymphokine-activated killer cells. Early IL-2 secretion by activated CD4+ T cells is also required for NK cells to secrete IFN- $\gamma$ upon infection with Leishmania major in mice [333], [323]. In humans, T cell derived IL-2 boosts NK cell in the LN, where CD4+ T cells and NK cells interact.

Numerous studies established that Foxp3 expressing regulatory $\mathrm{T}$ (Treg) cells that are critical for maintenance of adaptive immune tolerance, potently suppress NK cell responses in both human and murine systems [334], [335], [336].

It is likely that Treg cells control NK cell activity by regulating IL-2 production by activated T cells. Knowing that IL-2 deprivation is a prominent mechanism used by Treg to suppress spontaneous T cell activation [337], Kerdelis suggested that Treg could limit IL-2 availability thus controlling $\mathrm{T}$ cell activation and subsequentlytuning NK cell activity [323]. (Figure 15)
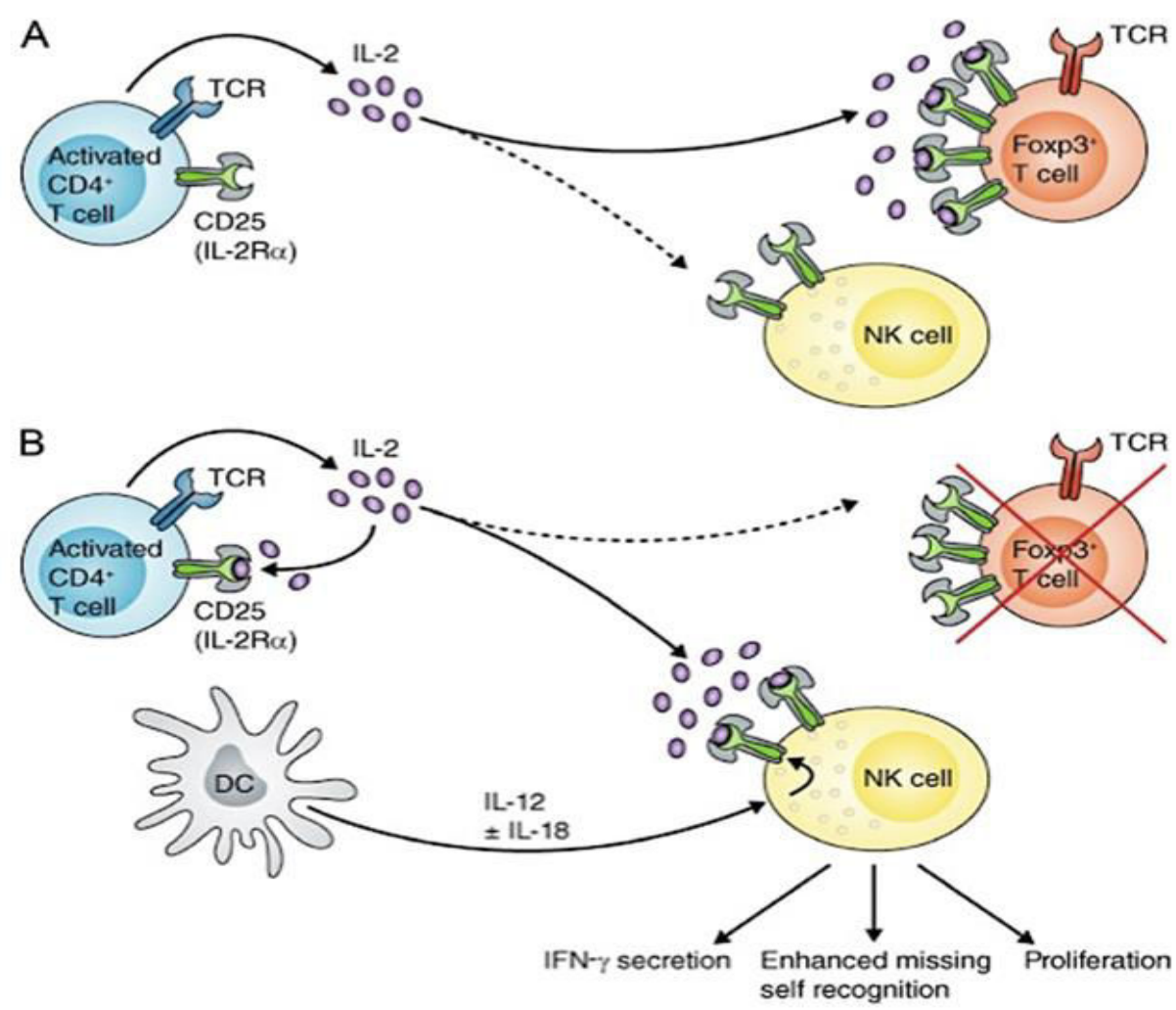

Figure 15: IL-2 dependent Treg cell-NK cell cross talk, (A) Treg deprive NK cells from CD4+ T cells-derived IL-2. (B) Upon loss of Treg, IL-2 becomes available and NK cells are released. (adapted from; Kerdiles et al, JEM, 2013) 
However, others suggest that Treg cells can regulate NK cell function through a transforming growth factor- $\beta$ (TGF- $\beta$ ) dependent mechanism in humans and mice [218]. A study by Smyth and his colleagues have shown that Treg suppression of NKG2D-mediated NK cell cytotoxicity in tumors is contact and TGF- $\beta$ dependent [335].

\section{G. NK cell memory}

Immunological memory is defined as the ability of the immune system to respond rapidly, thanks to the clonal expansion of its effector cells, which provide enhanced protection of the host against a previously encountered antigen [338]. Although it is a hallmark of adaptive immunity, there is accumulating evidence that NK cells possess some kind of memory ability [181].

In steady state conditions, the half-life of mature NK cells is estimated to be 17 days [339]. However, recent findings suggested that at least some mature NK cells can be long-lived and mount a robust recall response [181].

The first evidence for NK cell memory was observed in hapten-induced contact hypersensitivity studies in RAG-deficient micelacking T and B cells. Although previously thought to be mediated only by CD4+ T cells, NK cells could mediate the contact hypersensitivity responses after priming with a chemical hapten for at least a month and the responses were hapten specific. Interestingly, these hapten-specific memory NK cells resided in the liver and were marked by high levels of expression of surface Thy1 and CXCR6 [340], [341], [181] and [342] (Figure 16, right panel).

Later studies by Yokayama group provided the clues that NK could undergo clonal-antigen specific responses to MCMV infection. After infection with MCMV, only Ly49H+ NK cells underwent preferential expansion after recognizing MCMV m157 protein (Figure 16, left panel). In addition, when these cells were adoptively transferred into recepients infected with the virus, the Ly49H+ population underwent contraction after control of the virus, but memory NK cells could be detected in the recepient more than a month later [343], [344], [204].

Importantly, upon restimulation, these memory NK cells, like memory $\mathrm{T}$ lymphocytes, demonstrated enhanced cytolytic function and cytokine production compared to naive NK cells. Furthermore, this self-renewing population of NK cells could undergo subsequent rounds of proliferation and expansion following adoptive transfer to a second and even a third recipient. Although there is no yet unique marker for memory, these long lived NK cells generated during MCMV infection were all found to highly express KLRG1, an inhibitory 
receptor that recognizes E-cadherins [344].

MCMV-induced
$\begin{aligned} & \text { memory NK cell } \\ & \text { CycmV-infected cell }\end{aligned}$

Figure 16: Three paths towards becoming a memory NK cell, Right panel: Liver-restricted NK cells highly express CXCR6 and are induced by haptens and CXCL16, Center panel: Cytokineinduced NK cells do not require antigen presentation and are induced after exposure to IL-12, IL-15, IL-18. Left panel: MCMV-induced memory NK cells are IL-12 dependent, m157-specific. (adapted from; Min-Oo et al, Trends in immunology, 2013)

More recently, it has been reported that memory-like NK cells could be induced in vitro by exposure to IL-12 and IL-18. When adoptively transferred into naive recepients, these cytokine-activated NK cells could persist for at least a month and had the ability to produce cytokines upon restimulation [301]. (Figure 16, center panel)

Altogether, these findings suggest that NK cells appear to remember their past, a property previously considered true only for adaptive immune cells [181]. 


\section{H. Physiological Functions of NK cells}

\section{NK cells as regulatory cells}

NK cells act as regulatory cells to influence various other cell types such as DCs, T cells, B cells, and endothelial cells.

Functional interaction between NK and DCs results in their mutual regulation. This crosstalk can occur in the periphery or in secondary lymphoid tissue through cell contact or soluble factors. As discussed above, trans-presentation of IL-15 by IL-15R $\alpha$ on DCs stimulates the cytotoxic activity of NK cells and their ability to produce IFN- $\gamma$ [345], [332].

On the other hand, NK cells activated by tumor cells or virus-infected cells can prime DCs to produce IL-12, thus promoting TH1 responses. Intriguingly, NK cells can control DC maturation. Immature DCs are susceptible to NK cell-mediated cytolysis, whereas the mature DCs are protected. This effect is attributed to the level of MHC I molecules at the surface of immature DCs, triggered by signals delivered by activating receptor NKp33 and counterregulated by signals from the inhibitory receptor CD94-NKG2A [346], [347] and [329]. This DC editing may serve as quality control to guarantee the survival of DCs expressing sufficient co-stimulation molecules required for successful T cell priming [348].

Besides influencing DC function, NK cells can also shape adaptive immunity by directly acting on T and B cells [324]. Importantly, NK cells represent an early source of IFN- $\gamma$, which promotes proliferation and polarization of naive CD4 T cells towards a TH1 response in inflammed LN [217], [347]. Another mechanism how NK cells influence the emerging T cell response occurs indirectly via cross-presentation. After NK cell-mediated killing, the antigen released by target cells is uptaken by DCs, which in turn present it via MHC I to T cells resulting in increased CD8 T cell response. Same happens in human cells, IFN- $\gamma$ and TNF- $\alpha$ released by NK cells induce cross-presentation of tumor cell derived antigens by DCs, which subsequently induces tumor-specific CD8 T cell response [349].

In addition, NK cells can kill activated $\mathrm{T}$ cells unless they express sufficient amount of classical or non-classical MHC I, and can even suppress auto-reactive B lymphocytes in vitro. This supports previously reported protective role of NK cells in some autoimmune and inflammatory conditions [218], [350]. Hence, NK cells contribute to modulation of innate and adaptive immunity via either deletion of activated immune cells or through secretion of immunemodulatory cytokines. 


\section{Anti-tumoral and anti-viral activities of NK cells}

NK cells were first ascribed the anti-tumor property in 1975 when they were defined by their intrinsic capacity to kill tumor cells [174]. Subsequently, research revealed that these cytotoxic responses could also be elicited by virally infected non-tumor cells.

NK cells are unique for their ability to detect changes in tumor cells in the absence of inflammatory stimuli, and as such they directly react to kill tumors. Alternatively, they can also produce IFN- $\gamma$ or other cytokines which recruit DCs, T cells or B cells, which in turn provide a hand [347].

A robust correlation exists between the absence of $\mathrm{NK}$ and severe susceptibility to infections induced by viruses such as varicella-zooster virus, cytomegalovirus and herpes simplex virus [351]. Such studies were supported with the evidence of direct NK cell activation during viral infection when Mandelboim et al discovered that influenza hemagglutinin binds to NKp46 on human NK cells and induces cytotoxic activity [352].

In mice, studies demonstrated increased susceptibility or resistance to herpes virus MCMV after NK cell depletion or NK cell adoptive transfer respectively. In addition, defects in NK cell activity, such as decreased production of IFN- $\gamma$ or cytotoxicity, also rendered the mice more susceptible to MCMV infection [218], [353].

\section{NK cells and inflammatory diseases}

While studies in humans indicate that well-preserved NK cell numbers are markers of healthy aging, decreased NK cell levels or function are associated with chronic inflammatory diseases such as RA, multiple sclerosis, and psoriasis [354].

In a mouse model for multiple sclerosis, experimental autoimmune encephalomyelitis (EAE), depletion of NK cells exacerbated the disease, characterized by the occurrence of relapses. The reason behind that was increased T cell proliferation and cytokine production [355]. NK cell participated in the elimination of autoreactive CD4 $\mathrm{T}$ cells in a perforin-dependent manner besides TRAIL/TRAIL receptor pathway-induced apoptosis in autoreactive T cells. Another recent study confirmed that NK cells exert a direct cytotoxic effect on auto-antigen specific encephalitogenic T cells [356]. It is therefore suggested that NK cells regulate the immune responses that initiate EAE [357].

On the other hand, NK cells can also act as mediators of innate immunopathology [218]. In Type 1 diabetes (T1D), NK cells have been found in pancreatic islets only during infection and inflammation.The activating receptor, NKp46, is known to be associated with induction 
of T1D. Mice which are ablated of NKp46 are less prone to develop diabetes. Although the ligand for NKp46 is still unkown, it is stably expressed on pancreatic $\beta$ cells.But the fact that not all humans become diabetic is because NK cells are not commonly found in healthy pancreas. The outcome of $\beta$ cells recognition was NK cells degranulation and subsequent killing of the cells [348], [358], [359].

In RA, tissue NK cells have disease promoting functions. RA patients have higher proportions of activated and IFN- $\gamma$ secreting CD56 $6^{\text {bright }}$ subset in their joints compared to blood NK cells $[360,361]$. When isolated and co-cultured with patient's monocytes, these activated NK cells from the same patient were able to induce differentiation of monocytes to DCs [360], [362].

Conversely, in SLE, patients have low numbers of NK cells along with reduced cytotoxic activity. This deficiency in NK cell number and functions is attributed to clinical conditions such as nephritis and thrombocytopenia. Therefore, NK cells play a protective or disease controlling role to prevent SLE [363], [361]. 


$$
\text { -CHAPTER } 3 \text { - }
$$

\title{
Naturalkiller Cells
}

\author{
And
}

\section{Cardiovascular diseases}


NK cell deficiency has been reported in patients with coronary artery disease. In a 12-month follow-up study of coronary patients, deficit in NK cells was correlated with low-grade inflammation, whereas patients who had restored NK cell levels showed diminished inflammation, as assessed by decline in circulating IL-6 levels. However, it remains to be determined whetherNK cell deficit was causative or symptomatic [364], [365].

NK cells have been involved in cardiac repair after myocardial infarction (MI). MI is associated with an intense inflammatory response and activation of host innate immune program, which enhances inflammatory cell mobilization and cardiac repair and remodeling. Studies suggested that (c-kit+) BM-derived hematopeitic cells can repair damaged myocardium after MI through myogenesis and neovascularization [366]. The steel factor receptor (c-kit) is known to play a major role in $\mathrm{NK}$ cell production, maturation, and mobilization [367]. In their study, Ayach et al have shown that c-kit-mediated mobilization of bone marrow NK cells rescues heart post MI, contributing to improved remodelling and cardiac function [366]. A more recent study described the mechanism by which activated NK cells participate in cardiac angiogenesis and remodeling. In this study, it was demonstrated that NK cells interact with cardiac endothelial cells post MI, increase their proliferation, and promote angiogenesis through a mechanism involving $\alpha 4 \beta 7$ integrin and the inhibitory receptor KLRG1 [368].

NK cells have also been involved in the protection againt myocarditis. Myocarditis is an inflammatory disorder of the myocarduim, characterized by focal lesions consisting of necrotic myocytes with an infiltration of inflammatory cells. Although pathogenesis is still poorly understood, it is believed that myocarditis is induced by viral infections, mainly coxsackievirus B (CBV) and MCMV viruses [369]. Patients with acute myocarditis commonly present with acute cardiac failure. NK cells and CTL are the predominant cells infiltrating the myocardium. NK cells have been shown to kill virus infected myocytes in a perforin-dependent manner clearing the viral infection and providing some protection against virus-induced myocarditis [370].

\section{NK cells in Atherosclerosis}

NK cells have been detected in human [9], [371] and mouse [372] atherosclerotic lesions. In human advanced lesions, these cells were localized to regions near necrotic core and in shoulder regions [373]. And recently, they were identified near the fibrous cap and in close 
proximity to macrophages in mice atherosclerotic lesions [374]. Also, patients with severe atherosclerotic disease have highly circulating levels of NK cells [375]. In parallel, studies on elderly patients with peripheral arterial disease revealed increased number of total circulating NK cells along with decreased NK cytotoxicity on a per cell basis [376]. However, the lack of a good animal model that is selectively deficient in NK cells has limited the dissection of its true role in atherosclerosis and immunity.

Early experimental studies to determine the role of NK cells in atherosclerosis used the beige mutant mice that represent a mouse model of NK cell functional deficiency [377]. Two separate studies used beige mice. In the first study, conducted in beige mice fed a high fat diet containing cholate, no difference in lesion size was observed in beige mutant compared to control mice [378]. By contrast, in the second study where beige mice were inbred to $L d l r^{-/}$ (Lyst ${ }^{\text {beige }} \mathrm{Ldll}^{-/}$) mice, significant increase in lesion size was observed in beige $\mathrm{Ldll}^{-/}$mice fed a high-fat diet without cholate, compared to control $\mathrm{Ldll}^{-/}$mice, suggesting an atheroprotective role of NK cells [379]. The beige-mutant mice have a defect in lyososomal trafficking [380]. However, this defect in cell function is not restricted to NK cells, but also include neutrophils, macrophages, and smooth muscle cells, whose functional changes in lysosomal trafficking could lead to increased foam cell formation or apoptosis [381]. Therefore, it is unclear whether increase in atherosclerosis in Lyst $t^{b e i g e} L d l r^{-/}$mice is a result of impaired NK cell function.

Subsequently, Whitman et al.used chimeric mouse model where $L d l r^{-/}$mice received BM of Ly49A transgenic mice [372]. These mice overexpress the Ly49A receptor under the control of granzyme A promoter. Ly49A is an inhibitory receptor, which recognizes MHC class-I molecules (see above), expressed by NK cells and some CD8 T cells. Interaction of Ly49A with its ligand, MHC I molecule, inhibits NK cell activation, which provides a rationale for the absence of functional NK cells in these mice.

In this transgenic model, the number of $\mathrm{NK}\left(\mathrm{NK} 1.1^{+} \mathrm{CD}^{-}\right)$cells was markedly reduced in the spleen and other peripheral tissues and the number of NK1.1+ T cells was slightly reduced in the spleen. However, this phenotype, which is not totally understood, is linked to the expression of the ubiquitous transcription factor ATF2, raising the possibility of additional defects. Smaller size lesions were observed in $\mathrm{Ldll}^{-/}$recipients reconstituted with the BM of Ly49A transgenic mice. While the authors concluded that deficiency of NK cell activity was responsible for the observed effect, they did not exclude the possibility that $\mathrm{T}$ cells, specifically NKT and CD8 subsets, which are known to express granzyme A and whose 
functions are influenced by Ly49A [382], [383] could contribute to this proatherogencity [384]. Moreover, it is noteworthy that the NK cell activity was not assessed in this study [372].

More recently, Selathuraiet al. performed loss- and gain-of-function experiments in Apoe $e^{-/}$ mice to investigate the role of NK cells in atherosclerosis. In the loss-of-function experiments, treatment of Apoe $^{-/-}$mice with anti-asialo-GM-1 antibody significantly reduced atherosclerotic lesion development. However, the glycolipid asialo-GM1 is also expressed by several cell types, including myeloid cells, epithelial cells, and T-cell subsets [385], [386], [387], [388]. Hence, the selectivity of NK cell depletion with asialo-GM1 is hampered by the expression of asialo-GM1 by these other cell types.

This study was supported by gain-of-function experiments where NK cells were activated exvivo for 5 days with rIL-2 before being transplanted into immune-deficient mice. Adoptive transfer of ex-vivo pre-activated NK cells (WT, IFN- $\gamma^{-/-}$, perforin ${ }^{-/-}$, or granzyme ${ }^{-/-}$) into lymphocyte-deficient Apoe ${ }^{-/-}$mice Rag2-/- x Il2g-/- mice confirmed that transfer of WT or IFN- $\gamma^{-/-}$activated NK cells, but not perforin ${ }^{-/-}$or granzyme $^{-/-} \mathrm{NK}$ cells, promoted atherosclerosis and necrotic core development. The authors reached the conclusion that NK cells were atherogenic and their production of perforins and granzyme B contributed to the development of atherosclerosis [374]. Although these studies indicated that pre-activated NK cells can be proatherogenic, they do not help understanding the role of endogenous resting NK cells.

These contrasting observations reported until now could be due to different experimental settings. Therefore, further investigations are required to conclusively define the true role of NK cells in atherosclerosis development. How can selective depletion or activation of NK cells contribute to the development of the disease and what are the mechanisms employed?? Issues still need to be addressed to determine whether targeting NK cells could represent a useful therapeutic approach to attenuate atherosclerosis. 
II. OBJECTIVES 
During my thesis, I was interested in investigating the role of Natural Killer cells in atherosclerosis. Thus, I used state-of-the-art genetically modified mouse models to achieve my aims.

In summary, the aims of my thesis can be grouped in four main axes:

1. Examining the effect of selective NK cell depletion in atherosclerosis using $N c r 1^{\text {iCre }} R 26^{\text {Isl-DTA }}$ mouse model of NK cell deficiency.

2. Examining the effect of hyper-responsive NK cells using Noé mice, highly responsive to viral infections

3. Deciphering the selectivity of asialo-GM1-treatment to target NK cells in atherosclerosis context

4. Investigating the role of activated NK cells by an external inflammatory stimulus in atherosclerosis context

5. Analyzing NK cell localisation in human and mouse atherosclerotic plaques 


\section{RESULTS -}

Part 1 
Submitted Article:

\section{Genetic Depletion or Hyperresponsiveness of Natural Killer Cells do not affect Atherosclerosis development}

Wared Nour-Eldine ${ }^{1,2}$, Jeremie Joffre ${ }^{1}$, Bruno Esposito ${ }^{1}$, Jose Vilar $^{1}$, Eric Vivier ${ }^{3}$, Kazem Zibara $^{2,4}$, Hafid Ait-Oufella ${ }^{1}$, Ziad Mallat ${ }^{1,5}$, Sophie Ugolini ${ }^{3}$, Alain Tedgui ${ }^{1, *}$

${ }^{1}$ Institut National de la Santé et de la Recherche Médicale (Inserm), Unit 970, ParisCardiovascular Research Center, and Université Paris-Descartes, Paris, France.

${ }^{2}$ ER045, PRASE, Lebanese University, Beirut, Lebanon

${ }^{3}$ Aix Marseille Univ, CNRS, INSERM, Centre d'Immunologie de Marseille-Luminy, Marseille, France

${ }^{4}$ Biology Department, Faculty of Sciences-I, Lebanese University, Beirut, Lebanon

5 Department of Medicine, Division of Cardiovascular Medicine, University of Cambridge, Cambridge, United Kingdom

Running title: Natural Killer cells

Corresponding Author:

Alain Tedgui

Paris-Cardiovascular Research Center (PARCC), INSERM U970, 56 rue Leblanc, 75737 Paris cedex 15, France.

Email: alain.tedgui@inserm.fr 


\begin{abstract}
Objectives: Chronic inflammation is central in the development of atherosclerosis. Both innate and adaptive immunity are involved in this process. Although several studies have evaluated the functions of $\mathrm{NK}$ cells in experimental animal models of atherosclerosis, it is not yet clear whether NK cells behave as protective or pro-atherogenic effectors.

Methods and Results: One of the main caveats of previous studies was the lack of specificity in targeting loss- or gain-of-function of NK cells. Here, we used two selective genetic approaches to investigate the role of $\mathrm{NK}$ cells in atherosclerosis: 1) $\mathrm{Ncrl}^{i \mathrm{Cre} /+} \mathrm{R} 26^{\mathrm{IIIDT} /+}$ mice in which NK cells were depleted, 2) Noé mice in which NK cells are hyperresponsive. No difference in atherosclerotic lesion size was found in $\mathrm{Ldlr}^{-/}$mice transplanted with bone marrow cells from $N c r l^{i C r e} R 26 R^{l s l-D T A}$, Noé or WT mice. Also, no difference was observed in plaque composition in terms of collagen content, macrophage infiltration or the immune profile in blood and spleen, although Noé chimera had more IFN- $\gamma$-producing NK cells in comparison with WT mice. Then, we investigated the NK cell selectivity of anti-asialo GM1 anti-serum, which was previously used to conclude to the pro-atherogenicity of NK cells. Anti-asialo GM1 treatment decreased atherosclerosis in both $L d l r^{-/}$mice transplanted with $N c r I^{i C r e} R 26 R^{l s l-D T A}$ or WT BM, indicating that its anti-atherogenic effects are unrelated to NK cell depletion. Finally, to determine whether NK cells could contribute to the disease in conditions of pathological NK cell overactivation, we treated irradiated $\mathrm{Ldll}^{-/-}$mice reconstituted with either WT or $N c r I^{i C r e} R 26 R^{l s l-D T A} \mathrm{BM}$ with the viral mimic Poly(I:C) and found a significant reduction of plaque size in NK-cell deficient chimeric mice.
\end{abstract}

Conclusion: Our findings, using state-of-the-art mouse models, clearly demonstrate that NK cells have no direct effect on the natural development of hypercholesterolemia-induced atherosclerosis, but may play a role when an additional systemic NK cell overactivation occurs.

Key words: Atherosclerosis, Immune System, Inflammation, Natural Killer Cells, 


\section{Introduction}

Atherosclerosis is a chronic inflammatory disease characterized by accumulation of cholesterol, immune cells, and fibrous elements, forming atherosclerotic plaques in large and medium sized arteries ${ }^{1}$. Over the past 20 years, a large body of evidence supported the implication of innate and adaptive immunity in the development of the disease. Macrophages are the first immune cells to infiltrate developing lesions, as well as dendritic cells and $\mathrm{T}$ cells. However, less abundant cells such as NKT and NK cells are also present ${ }^{2}$. NK cells are bone marrow (BM)derived innate lymphocytes characterized by their unique ability to kill aberrant cells without prior sensitization ${ }^{3}$. They act by means of direct cytotoxicity against their targets and by producing large array of cytokines and chemokines, the latter contributing also to the initiation of antigen-specific immune responses and thus shaping of adaptive immunity ${ }^{4}, 5$. Regardless of their activation status and their localization NK cells express the NKp46 marker encoded by the Ncrl gene. NK cells have been identified within atherosclerotic plaques in humans as well as in mice. In advanced atherosclerotic plaques, they have been localized to necrotic cores, deep within the plaques and in shoulder regions ${ }^{6},{ }^{7}$. Chemoattractants such as CCL2 and CX3CL1 may be involved in their recruitment to the developing lesions ${ }^{8},{ }^{9}$. Directly addressing the role of NK cells in immunity and atherosclerosis has been challenging due to the lack of a good animal model of NK cell-deficiency. Earlier studies used the beige mutant mice, an accepted model of defective NK cell functional activity ${ }^{10}$, with opposing results. In the first study, Beige mutant mice fed a high fat diet (HFD) containing cholate showed no differences in atherosclerotic lesions compared to controls ${ }^{11}$. However, in a more recent study by Schiller and his colleagues, NK cells were ascribed an athero-protective role when beige mutant mice were bred to Ldlr-mice ${ }^{12}$. Beige mutation, which involves the lyst gene encoding a protein implicated in lysosomal trafficking, results in a complicated phenotype that goes beyond decreased NK cell activity ${ }^{13},{ }^{14}$. Defects in cell function may include, in addition to NK cells, neutrophils, macrophages, or smooth muscle cells (SMCs) ${ }^{15}$, making it unclear whether the increase in atherosclerosis in lystbeige $\mathrm{Ldll}^{-/}$mice was a consequence of defective NK cell function.

Subsequently, different results were observed using a Ly49A transgenic mouse model of NK cell deficiency ${ }^{7}$. In these transgenic mice the Ly49A cDNA was expressed under control of the mouse granzyme A genomic sequence, inducing the expression of the receptor on all NK cells and one-half of $\mathrm{T}$ cells ${ }^{16}$. Ly49A is an inhibitory receptor, which recognizes MHC class-I 
molecules ${ }^{17}$. In this transgenic model, the number of $\mathrm{NK}\left(\mathrm{NK} 1.1^{+} \mathrm{CD} 3^{-}\right)$cells was markedly reduced in the spleen and other peripheral tissues and the number of NK1.1+ T cells was slightly reduced in the spleen ${ }^{16}$. However, this phenotype, which is not totally understood, is linked to the expression of the ubiquitous transcription factor ATF2, raising the possibility of additional defects ${ }^{18}$. Smaller size lesions were observed in $\mathrm{Ldll}^{-/}$recipients reconstituted with the BM of ly49A transgenic mice ${ }^{7}$. While the authors concluded that NK cell deficiency was responsible for the observed effect, they did not exclude the possibility that T cells, specifically NKT and CD8 subsets; which are known to express granzyme A and whose functions are influenced by Ly49A ${ }^{19},{ }^{20}$ could contribute to this pro-atherogenicity. It is worth mentioning that NKT and CD8 T cells have been classified as proatherogenic cells ${ }^{21} 22$.

Lately, Selathurai et al. performed loss- and gain-of-function studies to assess NK cell function in atherosclerosis [22]. In the loss-of-function experiments, treatment of Apoe ${ }^{-/}$mice with antiasialo-GM-1 antibody significantly reduced atherosclerotic lesion development. This study was supported by gain-of-function experiments where adoptive transfer of NK cells (WT, IFN- $\square^{-/}$, or

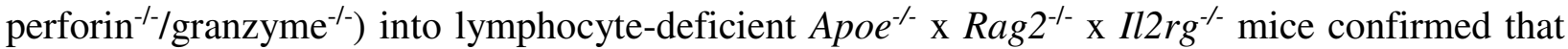
its cytotoxic effects are proatherogenic and promote necrotic core development ${ }^{23}$. However, the glycolipid asialo-GM1 is also expressed by several cell types, including myeloid cells, epithelial cells, and T-cell subsets. Hence, the selectivity of NK cell depletion with anti-asialo-GM1 antiserum has been questioned ${ }^{24}$.

Here, we used two state-of-the-art genetic approaches: 1) The $N c r 1^{i C r e} R 26 R^{l s l-D T A}$ mice in which NK cells are selectively depleted ${ }^{25}$ 2) The Noé mice in which NK cells are hyperresponsive ${ }^{26}$. $\mathrm{BM}$ from these mice were used to re-populate the hematopoietic system of lethally irradiated $L_{d l r^{-/}}$mice fed a high fat diet (HFD). Using these two genetic models, we provide strong evidence that NK cells do not significantly affect atherosclerosis development unless an additional inflammatory environment is induced. 


\section{Materials and Methods}

\section{Animals}

Experiments were conducted according to the guidelines formulated by the European Community for experimental animal use (L358-86/609EEC) and were approved by the Ethical Committee of INSERM and the French Ministry of Agriculture (agreement A75-15-32). $N c r I^{i C r e} R 26 R^{l s l-D T A}$ mice (deficient in NK cells) ${ }^{25}$ and Noé mice (high IFN- $\gamma$-producing- and CD107a-expressing- NK cells) ${ }^{26}$ have been generated and characterized by Eric Vivier's laboratory (CIML, Marseille, France).

The $N c r l^{i C r e} \mathrm{KI}$ mice ${ }^{27}$ were bred to $R 26 R^{l s l-D T A}$ mice to generate $N K 46^{i C r e /+} R 26^{l s l-D T A /+}$ mice referred as to $N c r I^{i C r e} R 26 R^{l s l-D T A}$ mice thereafter. In this model, the Cre-mediated removal of the floxed STOP codon, leads to the expression of the diphtheria toxin fragment A and consequent cell death. A near to complete absence of NK cells in the bone marrow and every tested organs (including the blood, thymus, lymph nodes, spleen, liver) was observed in these mice ${ }^{25}$. Of note, a deficiency in ILC1 (which also express NKp46) in the liver and in NKp46+ ILC3 in the small intestine was also observed in these mice.

The Noé mice were generated by random mutagenesis, in which a point mutation in the Ncrl gene impairs the cell surface expression of NKp46 receptor inducing higher capacity to produce IFN- $\gamma$ and to degranulate ${ }^{26}$.

Ten-week-old male C57BL/6J $\mathrm{Ldll}^{-/-}$mice were subjected to medullar aplasia by lethal total body irradiation $(9.5 \mathrm{~Gy})$. The mice were repopulated with an intravenous injection of BM cells isolated from femurs and tibias of sex-matched $N c r I^{i C r e} R 26^{l s l-D T A}$, Noé, or control C57BL/6J mice. After four weeks of recovery, mice were fed a pro-atherogenic high fat diet (HFD) containing $15 \%$ fat, $1.25 \%$ cholesterol, and $0 \%$ cholate for 8,12 , or 15 weeks.

In some experiments, 14-week-old chimeric male $L d l r^{-/}$mice, which received $\mathrm{BM}$ from $N c r I^{i C r e} R 26^{l s l-D T A}$ or control C57BL/6J mice, were treated every 5 days by intravenous injections of anti-asialo-GM-1 antibody (54 $\mu \mathrm{g}$ /injection), Wako chemicals, Richmond, VA, USA) or control serum (normal rabbit serum) for 8 weeks HFD. We used the same experimental protocol as previously described by Selathurai et al ${ }^{23}$.

For NK cell-activation experiments, 14-week-old chimeric male $L d l r^{-/}$mice, which received Ncrl ${ }^{i C r e} R 26^{l s l-D T A}$ or control C57BL/6J BM, were treated twice a week by intra-peritoneal injection of $100 \mu \mathrm{g}$ polyinosinic-polycytidylic acid (poly I:C), a synthetic analogue of dsRNA, TLR3 ligand (Invivogen, SD, USA) or saline (as a control) during 8 weeks HFD. 


\section{Quantification of atherosclerotic lesions}

Mice were anesthetized with isoflurane before sacrifice. Plasma cholesterol was measured using a commercial kit (DiaSys ${ }^{\circledR}$ Cholesterol FS*, Germany). Quantification of lesion size and composition was performed as previously described ${ }^{28}$. Briefly, the heart and ascending aorta were removed, perfusion-fixed in situ with $4 \%$ paraformaldehyde, then placed in phosphatebuffered saline (PBS)-30\% sucrose solution overnight, before being embedded in frozen optimal cutting temperature compound and frozen at $-70^{\circ} \mathrm{C}$. Afterwards, $10-\mu \mathrm{m}$ serial sections of aortic sinus were obtained (cut on cryostat). Lipids were detected using Oil Red O (Sigma-Aldrich, St. Louis, Missouri) coloration and quantified by a blinded operator using HistoLab software (Microvisions Instruments, Paris, France) ${ }^{29}$, which was also used for morphometric studies. En face quantification was used for atherosclerotic plaques along thoraco-abdominal aorta, as previously described ${ }^{28}$.

Collagen was detected using Sirius red staining. The presence of macrophages was determined using monoclonal rat anti-mouse macrophage/monocyte antibody (MOMA)-2 (specifically MAB1852).

At least 4 sections per mouse were examined for each immunostaining, and appropriate negative controls were used.

\section{Spleen cell culture and cytokine assays}

Cells were cultured in RPMI 1640 medium supplemented with Glutamax (Thermo Fischer Scientific), $10 \%$ fetal calf serum, $0.02 \mathrm{mM} \beta$-mercapto-ethanol and antibiotics. For cytokine measurements, splenocytes were cultured at $5^{*} 10^{5}$ cells/well for 24 hours and stimulated with lipopolysaccharide (LPS) $(1 \mu \mathrm{g} / \mathrm{ml})$ and IFN- $\gamma(100 \mathrm{UI} / \mathrm{ml})$ (Sigma). IL-6, IL-12, TNF- $\alpha$, IL1 $\beta$, IL-10, and IFN- $\gamma$ productions in the supernatants were measured using specific ELISA (R\&D Systems).

\section{Flow cytometry}

$\mathrm{NK}$ cells were identified as $\mathrm{CD}^{-} \mathrm{NK} 1.1^{+}$or $\mathrm{CD}^{-} \mathrm{NK} 1.1^{+} \mathrm{NKp} 46^{+}$. Monocytes were identified as $\mathrm{CD}_{11} \mathrm{~b}^{+} \mathrm{Ly}_{6 \mathrm{G}}{ }^{-}$or $\mathrm{CD} 11 \mathrm{~b}^{+} \mathrm{CD} 115^{\text {high }}$. Among them, classical monocytes were $\mathrm{Gr} 1^{\text {high }}$ (or Ly6C $\mathrm{C}^{\text {high }}$ ) and non-classical monocytes were $\mathrm{Gr} 1^{\text {low }}\left(\right.$ or Ly6 $\left.\mathrm{C}^{\text {low }}\right)$. Neutrophils were identified as 
$\mathrm{CD} 11 \mathrm{~b}^{+} \mathrm{CD} 115^{-} \mathrm{Ly}_{6 \mathrm{G}}{ }^{+}$. Regulatory $\mathrm{T}$ cells were considered as $\mathrm{CD}^{+} \mathrm{CD}^{+} \mathrm{CD} 25^{\text {high }} \mathrm{Foxp}^{+}$. $\mathrm{CD}^{+}, \mathrm{CD} 8^{+}$, and $\mathrm{NKT}\left(\mathrm{CD}^{+} \mathrm{NK} 1.1^{+}\right)$lymphocyte subsets were also analyzed.

Cells were labelled with FITC or PE-Cy7-conjugated anti-NKp46 (29A1.4), APC-conjugated anti-NK1.1 (PK136), PerCp-Cy5.5-conjugated anti-CD3e (145-2C11), FITC-conjugated antiCD4 (RM4-5), eFluor-450-conjugated anti-CD11b (M1/70), PE-conjugated anti-CD69 (H1.2F3), PE-Cy7-conjugated anti-CD115 (AFS98), PE-Cy7-conjugated anti-Foxp3 (FJK-16s), APCconjugated anti-CD25 (PC61.5), V500-conjugated anti-B220 (RA3-6B2), PE-conjugated anti-IL10 (JES5-16E3), PerCp-Cy5.5-conjugated anti-MHC II (M5/114.15.2) from eBiosciences, Alexa fluor 700-conjugated anti-CD8 (53-6.7), Brilliant Violet 421-conjugated anti-IFN- $\gamma$ (XMG1.2) and FITC-conjugated anti-CD107a (1D4B) from Biolegend, FITC-conjugated anti-Ly6C (AL21), PE-conjugated anti-Ly6G (1A8), PerCp-Cy5.5-conjugated Gr-1 (RB6-8C5), PE-Cy7conjugated anti-CD11c (HL3), APC-conjugated anti-IgM (11/41) from BD Biosciences. For blood staining, erythrocytes were lysed using BD FACS lysing solution (BD Biosciences). For intracellular cytokine staining, lymphocytes were stimulated in vitro with leukocyte activation cocktail (BD Biosciences) according to manufacturer's instructions for $4 \mathrm{~h}$. Surface staining was performed before permeabilization using Foxp3 staining buffer kit (eBiosciences) and intracellular staining.

Forward scatter (FSC) and side scatter (SSC) were used to gate live cells excluding RBC, debris, and cell aggregates in total blood cells, splenocytes, and BM. Single cell suspensions stained with fluorophore-conjugated antibodies were acquired using an LSRII Fortessa (BD) flow cytometer and analyzed with FlowJo software (Miltenyi).

\section{NK Cell Stimulation}

For specific NK cell stimulation, spleen cell suspensions were dispensed into 96-well 2HB Immulon plate previously coated with $25 \mu \mathrm{g} / \mathrm{ml}$ of purified anti-NK1.1 antibody or $10 \mu \mathrm{g} / \mathrm{ml}$ of anti-NKp46 antibody. Anti-CD107a antibody was added in the presence of monensin for 4 hours. For IL-12/18 stimulation, spleen cells were incubated with $20 \mathrm{ng} / \mathrm{ml} \mathrm{IL-18}$ and $25 \mathrm{ng} / \mathrm{ml} \mathrm{IL-12}$ (R\&D systems) for 4 hours in the presence of monensin. Cells were surface stained and intracellular IFN- $\gamma$ was revealed.

LIVE/DEAD Fixable Aqua Dead Cell Stain Kit (Thermo Fischer Scientific, L34957) was used as a viability dye and the percentage of IFN- $\gamma$-producing and CD107a-expressing NK cells was detected by Flow cytometry as mentioned above. 


\section{Quantitative real-time PCR}

Total ribonucleic acid (RNA) from spleen or abdominal aorta was extracted using Trizol reagent (Invitrogen). Quantitative real-time polymerase chain reaction (PCR) was performed on an ABI Prism 7700 Sequence Detection System (Thermo Fisher Scientific, Inc.) in duplicates. Cycle threshold for GAPDH was used to normalize gene expression of samples. Relative expression was calculated using the 2-delta-delta computed tomography (CT) method followed by geometric average, as recommended ${ }^{30},{ }^{31}$. The following primer sequences were used: IL-10 (F: 5' AAG TGA TGC CCC AGG CA 3'; R: 5' TCT CAC CCA GGG AAT TCA AA 3'), perforin (F: 5' ACA GTA GAG TGT CGC ATG TAC AGT TT 3'; R: 5' GAG GGC TCT GAG CGC CTT TTT GAA 3'), granzyme B (F: 5' ACT CTT GAC GCT GGG ACC TA; R: 5' AGT GGG GCT TGA CTT CAT GT 3'), IFN- $\gamma$ (F: 5' TCA AGT GGC ATA GAT GTG GAA AGA A 3'; R: 5' TGG CTC TGC AGG ATT TTC ATG 3'), TGF- $\beta$ (F: 5' CGG CCG GAA ATT CCC AGC TTC T 3'; R: 5' GTG AGA CGG GCT TCG GGG TCA T 3'), TNF- $\alpha$ (F: 5' GAT GGG GGG CTT CCA GAA CT 3'; R: 5' CGT GGG CTA CAG GCT TGT CAC 3'), IL1ß (F: 5' GAA GAG CCC ATC CTC TGT GA 3'; R: 5' GGG TGT GCC GTC TTT CAT TA 3'), and IL-6 (F: 5' AAA GAC AAA GCC AGA GTC CTT CAG AGA GAT 3'; R: 5' GGT CTT GGT CCT TAG CCA CTC CTT CTG T 3'). PCR conditions were $10 \mathrm{~min}$ at $95^{\circ} \mathrm{C} ; 42$ cycles of $95^{\circ} \mathrm{C}$ for 15 $\mathrm{s}, 60^{\circ} \mathrm{C}$ for $30 \mathrm{~s}$, and a final extension of $72^{\circ} \mathrm{C}$ for $30 \mathrm{~s}$.

\section{Statistical analysis}

Values are expressed as mean +/- SEM. Differences between values were evaluated using nonparametric Mann-Whitney test or One-way ANOVA with Bonferroni correction to compare more than two groups. All these analysis were performed using GraphPad Prism version 5.0b for Mac (GraphPad Software) and values were considered significant at $\mathrm{P}<0.05$. 


\section{Results}

\section{Genetic NK cell depletion does not alter the development of atherosclerosis}

To assess the direct role of NK cells in the development of atherosclerosis, we performed BM transplantation experiments using either control (WT) or $N c r I^{i C r e} R 26^{l s l-D T A} \mathrm{BM}$ cells to repopulate lethally irradiated male atherosclerosis prone- $L d l r^{--}$mice.

After 4 weeks of recovery, mice were put on high-fat diet (HFD) for 8, 12, or 15 weeks. Firstly, we confirmed NK cell depletion in spleen (Fig. 1A-B), blood (Fig. S1), and BM (data not shown) of chimeric Ncrl ${ }^{i C r e} R 26^{l s l-D T A} \rightarrow L d l r^{-/}$mice. As expected, the frequencies of other leukocyte populations in the spleen (Fig. 1C), blood (Fig. S1), and BM (data not shown) were not different between groups of chimeric mice. Data shown in Fig. 1 corresponds to 8-week HFD-fed mice. Data were similar in 12- and 15-week HFD fed-mice (data not shown). Circulating cholesterol levels and animal weights were similar between the two groups of mice (Table 1). Interestingly, NK cell deficiency did not alter lesion size in aortic root and thoracic aorta after 8 (Fig. 2A-B), 12, or 15 weeks of HFD (Fig. 2C-D). Similarly, no effect was observed on atherosclerosis development in female $\mathrm{Ldll}^{-/}$mice reconstituted with BM of control or $\mathrm{Ncr}{ }^{i C r e} \mathrm{R} 26^{\text {lsl-DTA }}$ mice and put on HFD for 8 weeks (Fig. S2 A, B, C). To determine whether plaque composition was affected by NK cell depletion, we analyzed macrophage content (Fig. S2 D, E), collagen content (Fig. S2 F, G) and necrotic core (data not shown), but found no significant differences between groups.

Furthermore, we investigated the immune-inflammatory response in chimeric $\mathrm{Ldlr}^{-/}$mice since NK cells are known to produce cytokines in response to infection or inflammation ${ }^{32}$. The expression levels of IL-6, IL-10, IL-1 $\beta$, IL-12p70, TNF- $\alpha$, and TGF- $\beta$ mRNA measured in the spleen, were not different between control and NK cell-deficient chimeric mice (Fig. S3 A). LPS and IFN- $\gamma$-stimulated splenocytes from chimeric control or $N c r I^{i C r e} R 26^{l s l-D T A}$ mice also showed no difference in inflammatory phenotype with same levels of IL-6, IL-10, and TNF- $\alpha$ (Fig. S3 B).

\section{Hyperresponsive NK cells do not promote atherosclerosis development}

We next addressed the role of NK cell hyperresponsiveness in atherosclerosis by using Noé mice, in which a point mutation in the Ncrl gene impairs the cell surface expression of the NKp46 
receptor inducing a higher capacity to produce IFN- $\gamma$ and to degranulate ${ }^{26}$. We performed BM transplantation experiments using Noé or control (C57/B6J) mice to reconstitute the BM of male $\mathrm{Ldlr}^{-/-}$mice. After 8 weeks of HFD, an intact NK cell population $\left(\mathrm{CD} 3^{-} \mathrm{NK} 1.1^{+}\right)$was detected in Noé chimeric mice compared to controls (Fig. 3 A, B, C). The frequencies of other leukocyte populations were also the same in spleen and blood of mice reconstituted with the WT or Noé BM (Fig. 4S E and F). Interestingly, a higher frequency of IFN- $\gamma$-producing NK cells was observed in chimera mice reconstituted with the Noé BM. However, no difference in plaque size, aortic root and thoracic aorta (Fig. $3 \mathrm{D}$ to $\mathrm{G}$ ) or plaque composition in terms of macrophage accumulation or collagen content (Fig. S4 A to D) was observed between groups. No difference in immune-inflammatory response was detected either (data not shown). This series of experiments was repeated twice, and similar results were found. There were no differences in plasma cholesterol levels or mice body weight between Noé and control chimeric mice (Table 1). These data show that NK cell hyperresponsiveness observed in Noé mice is not sufficient to affect the development of atherosclerosis.

\section{Anti-asialo GM-1-mediated decrease in atherosclerosis is NK cell-independent}

The findings with the above two experiments were unexpected, given that the latest study on the role of NK cell in atherosclerosis came up with the conclusion that NK cells are pro-atherogenic through their production of perforin and granzymes ${ }^{23}$. Hence, we revisited the experimental model of Selathurai et al. ${ }^{23}$, in which the authors used anti-asialo-GM1 antibody to deplete NK cells in Apoe $e^{-/}$mice. We treated control $\left(W T \rightarrow L d l r^{-/}\right)$or NK cell-deficient $\left(N c r l^{i C r e} R 26^{l s l-D T A} \rightarrow\right.$ $L d l r^{-/}$) chimeric mice either with anti-asialo-GM1 or control serum every other 5 days during the 8 weeks HFD.

Initially, we examined the effectiveness of anti-asialo-GM1 treatment for depleting NK cells in peripheral blood. A single dose of anti-asialo-GM1 antibody was sufficient to deplete NK cells within 5 days by greater than $80 \%$ in WT chimeric mice (Fig. 4A). NK cells are absent in $N c r{ }^{i C r e} R 26^{l s l-D T A}$ chimeric mice. However, anti-asialo-GM1 treatment also depleted CD8+ T and NKT populations in both control and $N c r 1^{i C r e} R 26^{I s l-D T A}$ chimeric mice compared to control groups treated with control serum (Fig. 4A).

Similar effects in blood and spleen cells were observed in anti-asialo-GM1-treated chimeric mice after 8 weeks of HFD. Anti-asialo-GM1 treatment led to almost $80 \%$ reduction of NK cells in control mice, between $60 \%$ and $80 \%$ reduction in NKT cells in control and NK cell-deficient chimeric mice, respectively, and 30\% reduction in CD8 T cells in both groups (Fig. 4B). 
Although anti-asialo-GM1 treatment seemed to be less effective in depleting NKT and CD8 ${ }^{+} \mathrm{T}$ cell populations after 8 weeks HFD in the spleen, the trend towards a smaller size population persisted (Fig. S5 A). There were no differences in the percentage of monocytes but we observed a trend of decrease in blood (Fig. 4B) and spleen (data not shown) neutrophils.

Importantly, IFN- $\gamma$-producing $\mathrm{CD}^{+} \mathrm{T}$ cell population was significantly decreased by almost $50 \%$ in anti-asialo-GM1-treated $N c r I^{i C r e} R 26^{I s l-D T A}$ chimeric mice with a trend also in control antiasialo-GM1-treated chimeric mice $(\mathrm{P}=0.077)$, while no significant differences were observed in IFN- $\gamma$ producing $\mathrm{CD}^{+} \mathrm{T}$ cells (Fig. S5 B).

Lesion size decreased significantly by $42 \%$ in anti-asialo-GM1-treated control chimeric mice compared to mice treated with control serum (Fig. 5). However, more interestingly, similar effects were observed in NK cell-deficient chimeric mice with $68 \%$ reduction in aortic root lesion size in anti-asialo-GM1-treated $N c r 1^{i C r e} R 26^{l s l-D T A}$ chimeric mice compared to mice treated with control serum (Fig. 5), despite similar plasma cholesterol levels (Table 1). Therefore, we concluded that reduction of atherosclerotic lesions by anti-asialo-GM-1 treatment was NK cellindependent.

\section{NK cells exacerbates atherosclerosis development in inflammatory conditions}

Finally, we addressed the role of NK cells on the development of atherosclerosis in inflammatory conditions in which these cells are known to be activated. To do so, we used polyinosinicpolycytidylic acid [Poly(I:C)], which mimics a viral infection. Control $\left(W T \rightarrow L d l r^{-/}\right)$or NK celldeficient $\left(N c r I^{i C r e} R 26^{I s l-D T A} \rightarrow L d l r^{-/}\right)$chimeric mice were treated repeatedly with $100 \mu \mathrm{g}$ Poly(I:C) twice a week, while another control group $\left(W T \rightarrow L d l r^{-/}\right.$mice) was treated in parallel with saline over 8 weeks HFD.

Interestingly, after 8 weeks of HFD, Poly(I:C)-treatment of control mice significantly increased CD69 and CD107a expression on NK cells compared to control mice that received saline, although the increase in frequency of IFN- $\gamma$-producing NK cells is not significant. As expected, the percentage of NK cells significantly decreased by almost 50\% in the spleen of Poly(I:C)treated compared to saline-treated chimeric mice reconstituted with WT BM (Fig. 6 A). Moreover, the expression of perforin, granzyme B, and IFN- $\gamma$ mRNA was significantly enhanced after Poly(I:C) treatment (Fig. 6B), which further confirms NK cell activation in response to Poly(I:C) treatment.

Although no change in aortic root plaque size was observed in Poly(I:C)-treated compared to saline-treated $\left(W T \rightarrow L_{d l l}^{-/}\right)$chimeric mice, NK cell deficiency in Poly(I:C) treated chimeric 
mice markedly decreased lesion development compared with Poly(I:C) treated WT controls (Fig. 7). Reduction in atherosclerosis was associated with reduction in macrophage infiltration in $\left(N c r I^{i C r e} R 26^{l s l-D T A} \rightarrow L_{d l l}^{-/}\right)$Poly(I:C)-treated mice compared to Poly(I:C)-treated control mice (Fig. S6 A). No change in collagen content was detected (Fig. S6 B). No differences in body weight or serum cholesterol levels were observed between the 3 groups of mice (Table 1). Hence, NK cells might be proatherogenic when activated in a systemic inflammatory context. 


\section{Figures and Figure Legends}
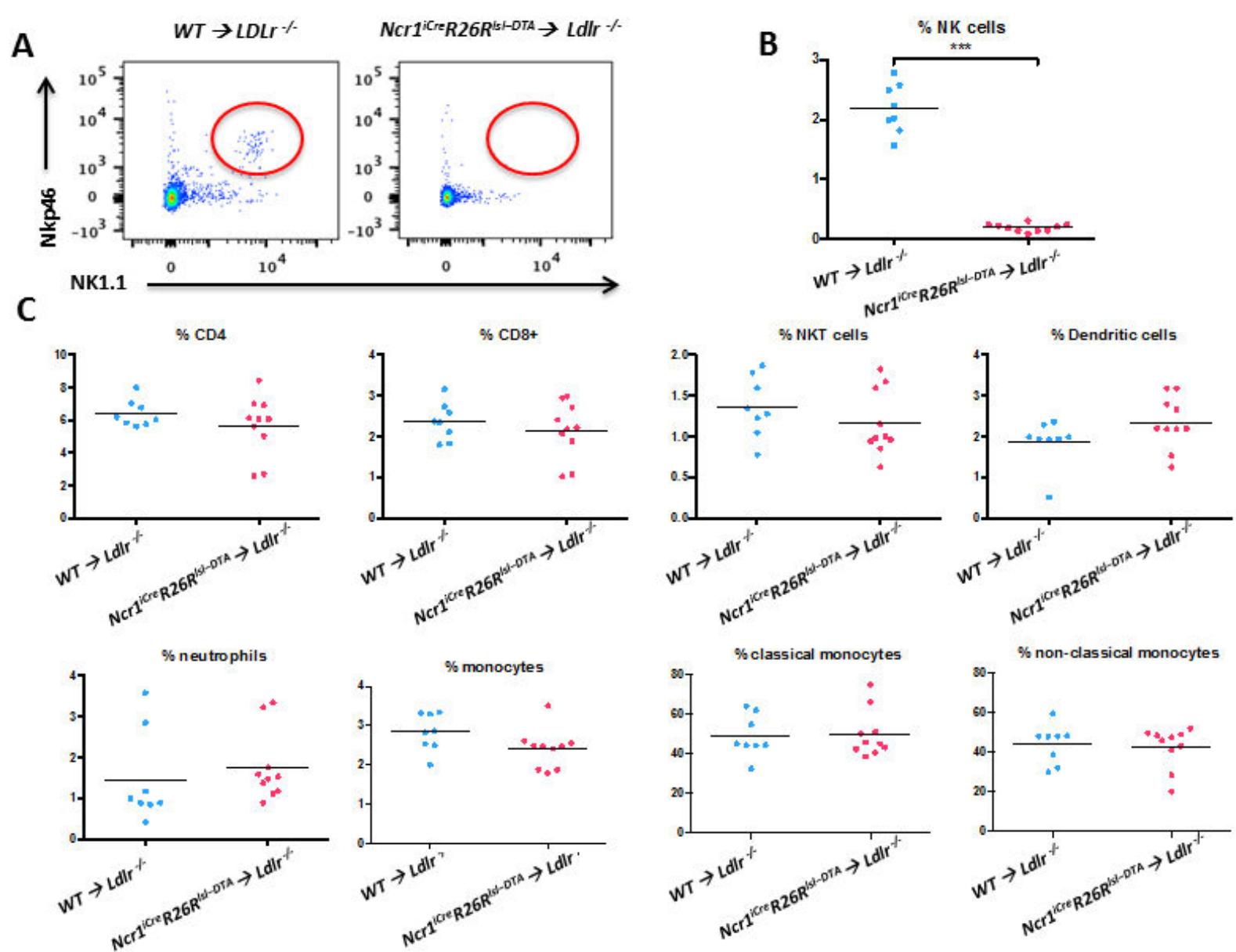

Figure 1: (A and B) Efficiency of NK cell $\left(\mathrm{CD3}^{-} \mathrm{NK}^{-1}{ }^{+} \mathrm{NKp46}^{+}\right)$depletion in spleen (gated on total viable splenocytes) of male $L d l r^{-/-}$mice reconstituted with the BM of NK cell-

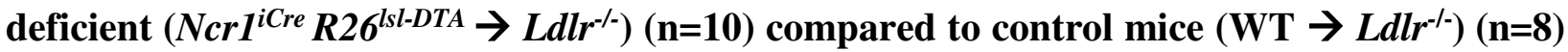
after 8 weeks of high fat diet (HFD). ***p $<0.0001$ (C) Representative quantitative analysis of the percentage of myeloid (neutrophils, monocytes (classical and non-classical), and dendritic cells) and lymphoid (CD4+, CD8+, and NKT cells) populations in the spleen of control and NK cell-deficient chimeric mice assessed by flow cytometry, after 8 weeks of HFD. 


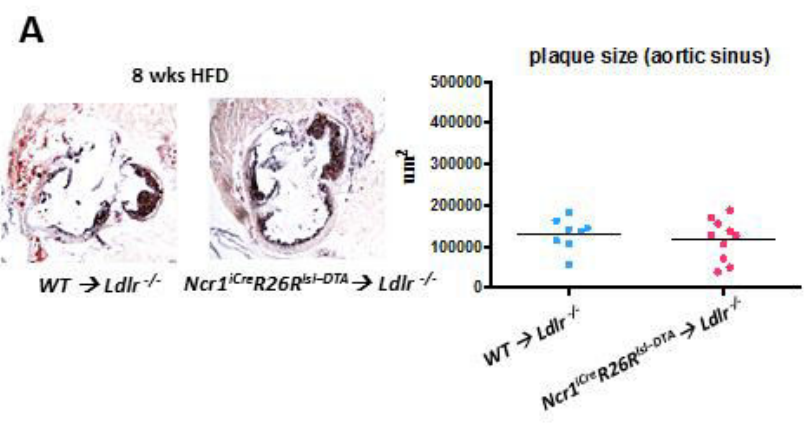

B

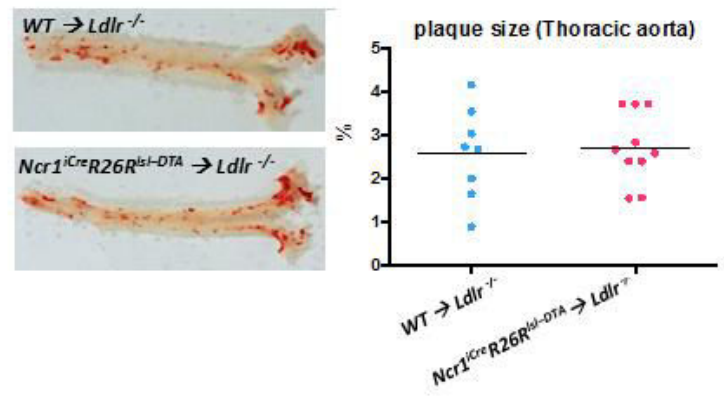

C

D
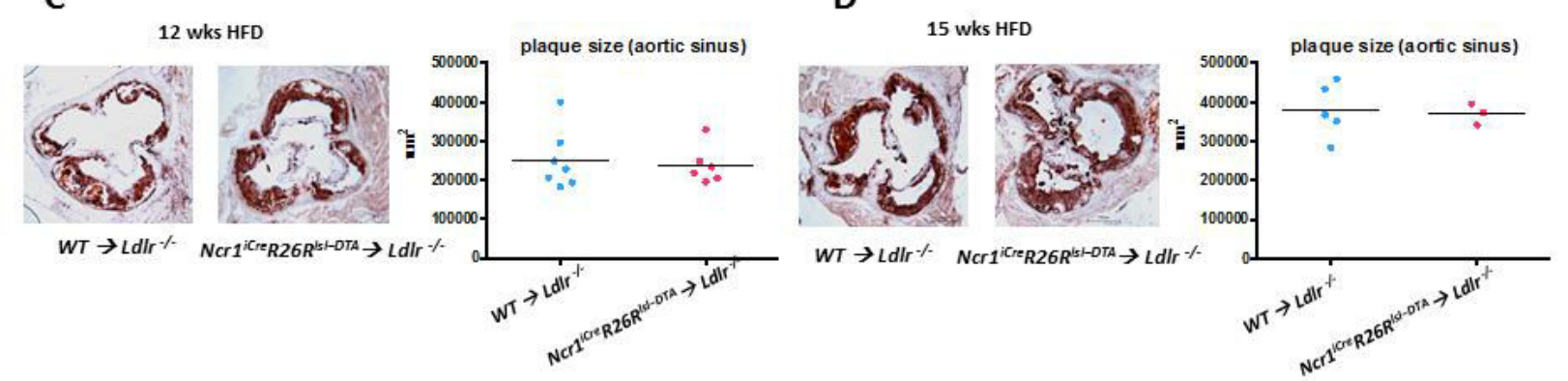

Figure 2: Representative photomicrographs of Oil red $\mathrm{O}$-staining and quantification of lesion size in aortic sinus (A) or thoracic aorta (B) of male controls (WT $\left.\rightarrow L d l r^{-/-}\right)(\mathrm{n}=8)$ and NK cell-deficient $\left(\mathrm{Ncrl}^{i C r e} \mathrm{R}^{\mathrm{i} 6^{I s l-D T A}} \rightarrow \mathrm{Ldlr}^{-/-}\right)(\mathrm{n}=10)$ mice after 8 weeks (A-B), 12 weeks (n=7 - 6, respectively) (C), or 15 weeks $(n=5-3$, respectively) HFD (D). 
A

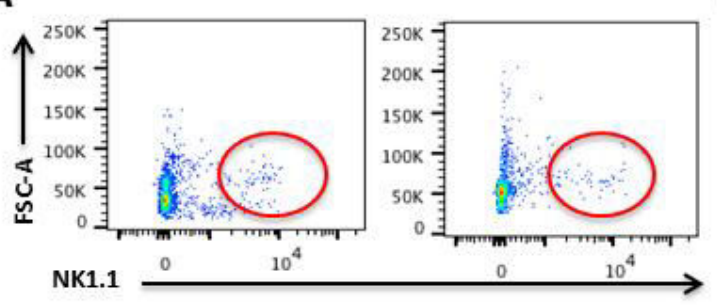

D

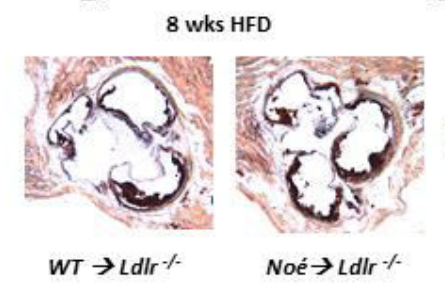

E

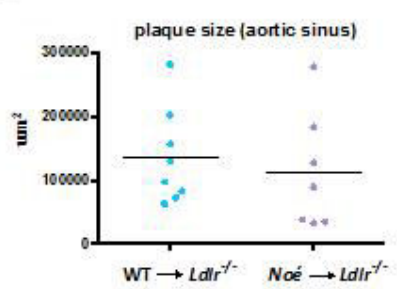

B

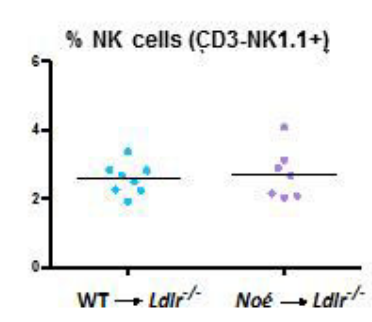

$\mathbf{F}$

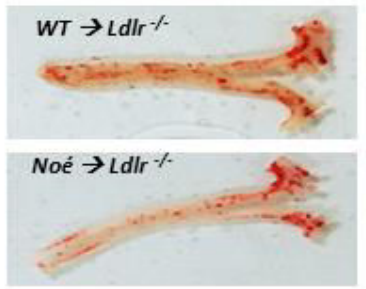

C

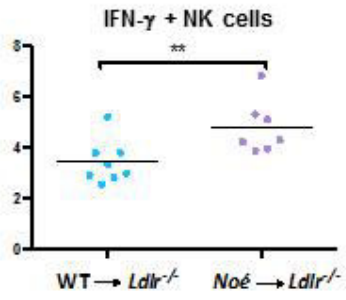

G

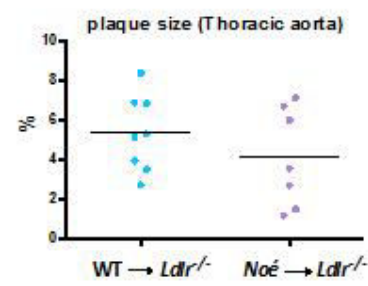

Figure 3: Representative Flowcytometry (A) and quantitative (B) analysis of the percentage

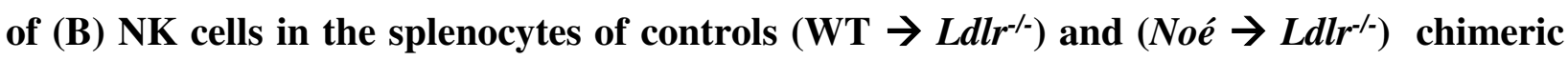
mice gated on $\mathrm{CD3}^{-} \mathrm{NK} 1.1^{+}$cells and (C) IFN- $\gamma$-producing NK cells gated on CD3-NK1.1 ${ }^{+}$ cells. Representative photomicrographs of Oil red O-stained atherosclerotic lesions in the aortic sinus (D) and thoracic aorta (F) of male controls $(n=8)$ and Noé chimeric mice $(n=7)$, along with their corresponding quantifications ( $E$ and $G$ respectively) after 8 weeks HFD. 

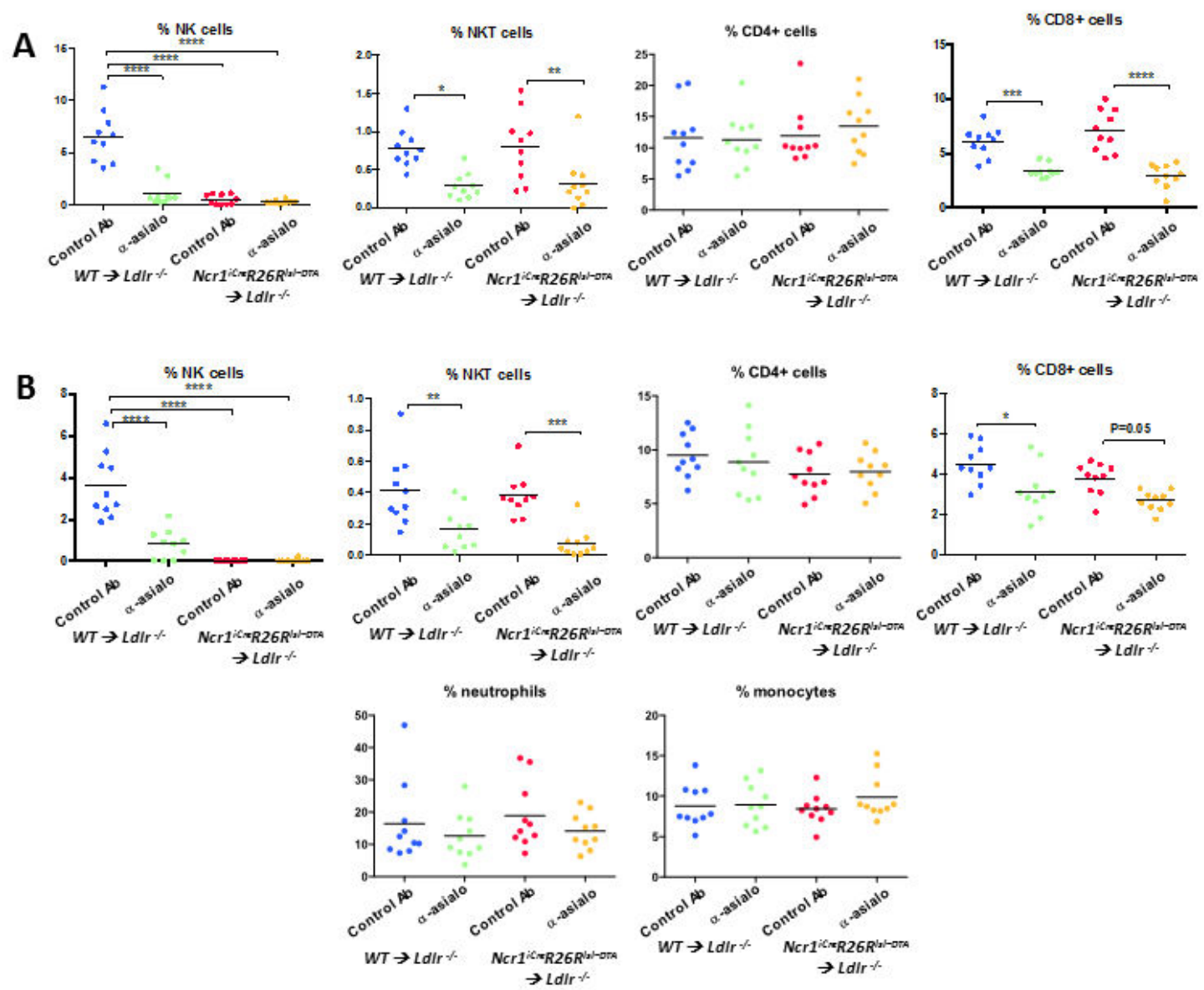

Figure 4: (A) Representative quantitative analysis of blood cells' percentage (NK, NKT, CD4, and CD8 cells) 5 days after commencing treatment with $\alpha$-Asialo-GM1 in controls $\left(\mathrm{WT} \rightarrow \mathrm{Ldlr}^{-/-}\right)$and NK cell-deficient $\left(\mathrm{NcrI}^{i C r e} \mathrm{R}^{\mathrm{C} \sigma^{I l-D T A}} \rightarrow \mathrm{Ldlr}^{-/-}\right)$mice treated with either $\alpha-$ Asialo-GM1 or control serum.

(B) Representative quantitative analysis of the percentage of myeloid and lymphoid cells in blood of same groups after 8 weeks HFD. 
A

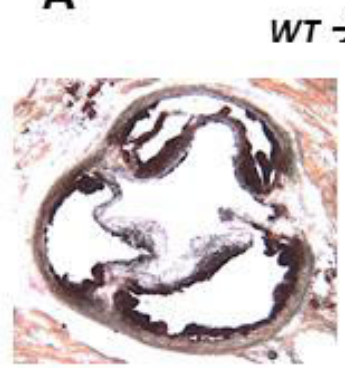

control Ab

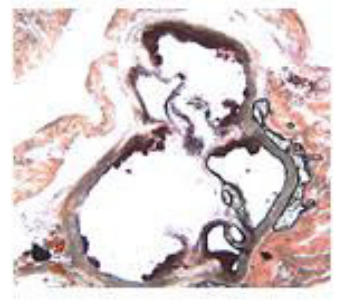

$\alpha$-asialo GM1
B

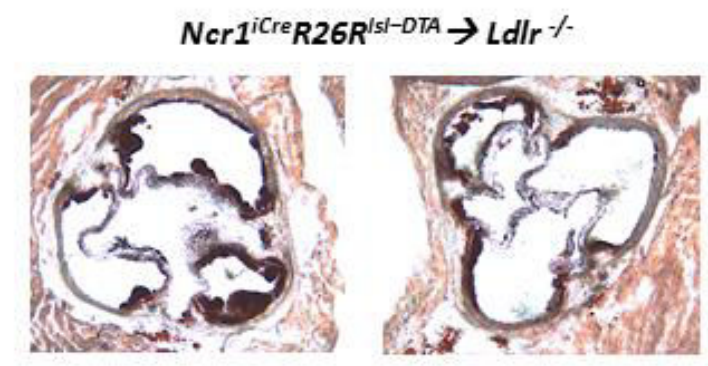

control $A b$

$\alpha$-asialo GM1

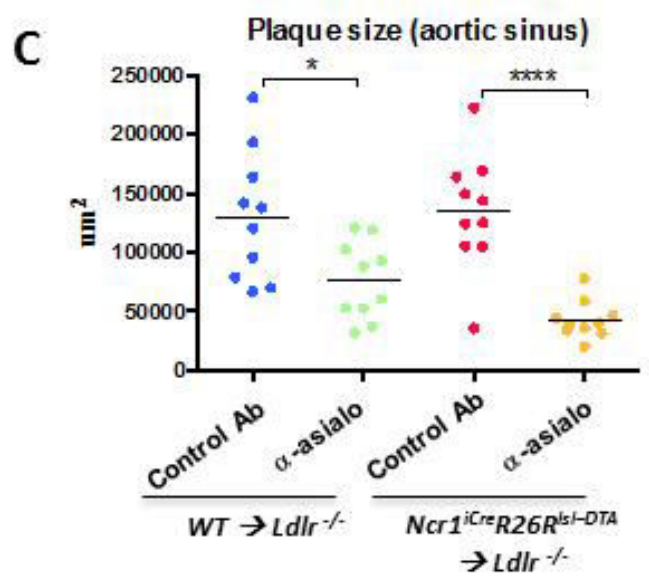

Figure 5: Representative photomicrographs of Oil red O-staining (A and B) and quantification of lesion size in left aortic sinus (C) after 8 weeks of HFD of male controls (A) and NK cell-deficient mice (B) treated with either $\alpha$-Asialo-GM1 or control serum ( $n=10$ per group). 
A

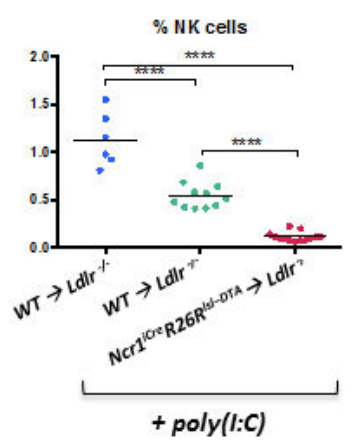

B

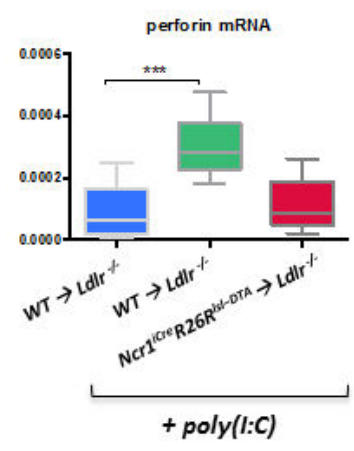

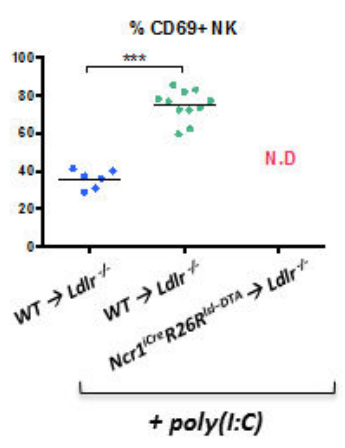
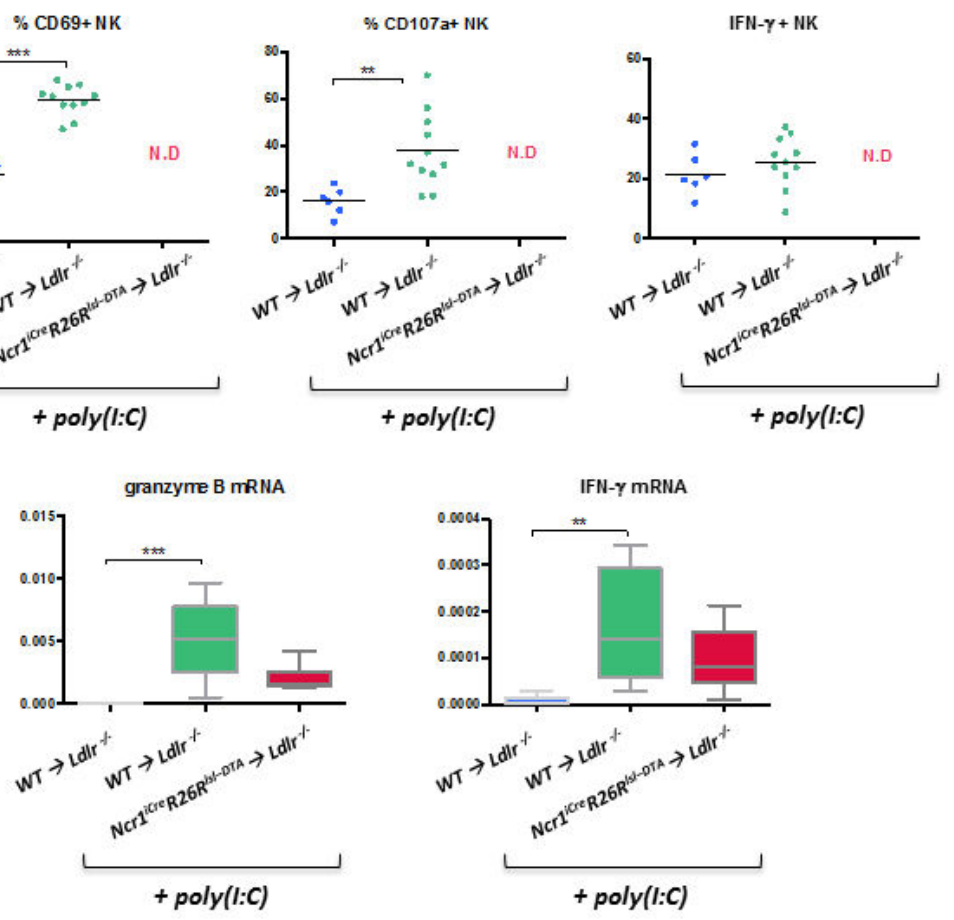

Figure 6: (A) Representative quantitative analysis of flow cytometry-based staining of NK cells (gated on $\mathrm{CD3}^{-} \mathrm{NK}^{-1} 1^{+}$), CD69+ $\mathrm{NK}$ cells (gated on $\mathrm{CD3}^{-} \mathrm{NK} 1.1^{+}$), IFN- $\gamma+\mathrm{NK}$ cells (gated on CD3-NK1.1 ${ }^{+}$), and CD107a+ NK cells (gated on CD3-NK1.1 ${ }^{+}$) in the three groups of mice: controls (WT $\left.\rightarrow \mathrm{Ldll}^{-/}\right)$treated with saline $(\mathrm{n}=6)$ or poly(I:C) $(\mathrm{n}=11)$ and NK celldeficient $\left(\mathrm{NcrI}^{\text {iCre }} \mathrm{R}^{26^{I s l-D T A}} \rightarrow \mathrm{Ldlr}^{-/}\right)$mice treated with poly(I:C) $(\mathrm{n}=13)$.

(B) Quantification of perforin, granzyme B, and IFN- $\gamma$ mRNA expression in the spleens of the 3 groups of chimeric mice. 
A

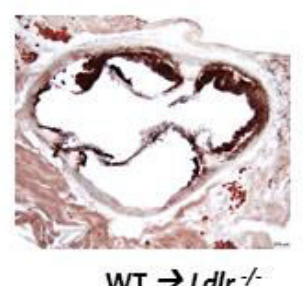

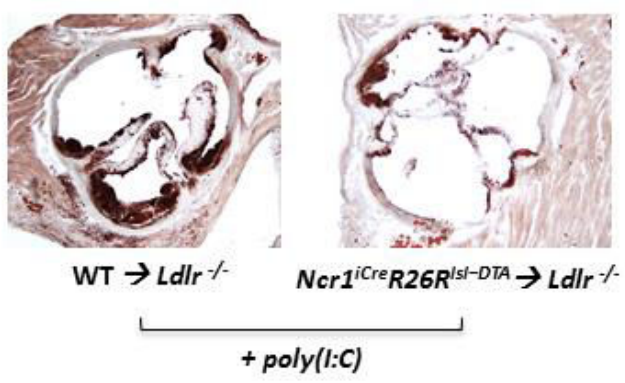

B

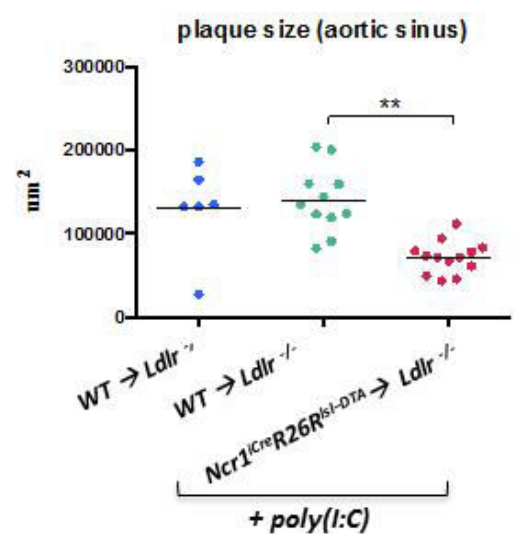

Figure 7: Representative photomicrographs of Oil red O-staining (C) and quantification of lesion size in left aortic sinus (D) after 8 weeks of HFD in the three groups 

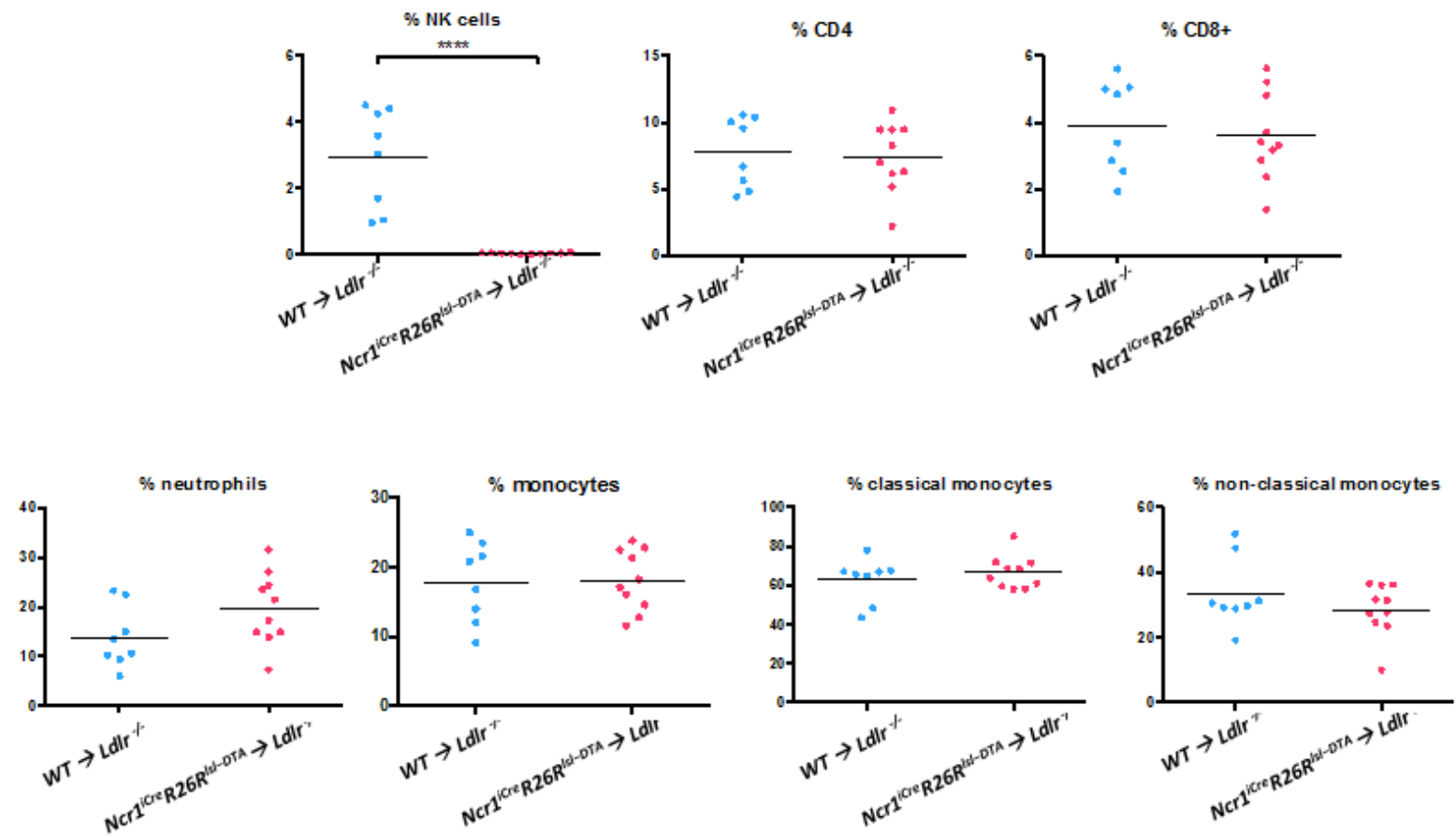

Supplementary Figure 1: Representative quantitative analysis of the percentage of myeloid (neutrophils, monocytes: classical and non-classical) and lymphoid (CD4+ and CD8+) populations in the blood of control $(n=8)$ and NK cell-deficient $(n=10)$ male chimeric mice assessed by flow cytometry, after 8 weeks of HFD. $* * * \mathbf{p}<0.0001$ 
A

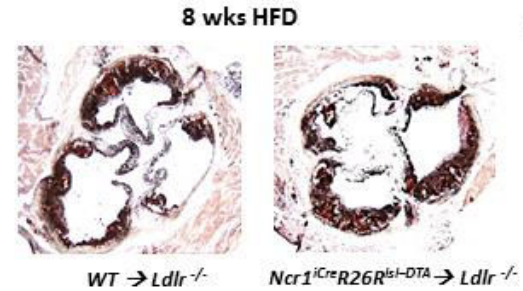

D

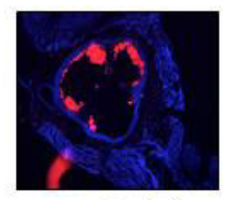

$W T \rightarrow$ Ldlr $\%$
B

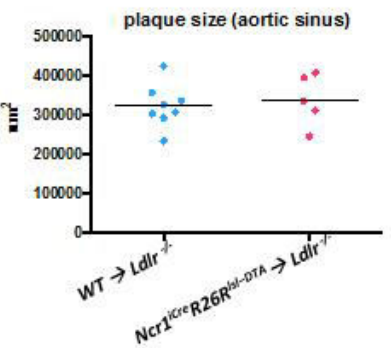

$\mathbf{F}$

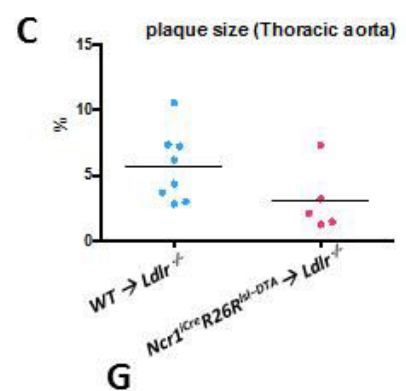

G

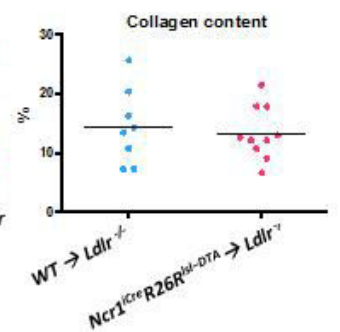

Supplementary Figure 2: Representative photomicrographs of Oil red O-stained atherosclerotic lesions (A) and quantification of mean lesion size in aortic sinus (B) and thoracic aorta $(C)$ of female controls $(n=8)$ and NK cell-deficient $(n=5)$ mice after 8 weeks HFD.

Representative photomicrographs (D) of controls and NK cell-deficient mice and quantitative analysis (E) of macrophage accumulation (macrophage plus monocyte antibody, MOMA staining) in atherosclerotic lesions of male chimeric $\mathrm{Ldll}^{-/-}$mice, 8 weeks HFD. Representative photomicrographs $(F)$ of controls $(n=8)$ and NK cell-deficient $(n=10)$ mice and quantitative analysis (G) of lesion collagen content (Sirius red) of male chimeric $L d l r^{-/-}$mice, 8 weeks HFD. 

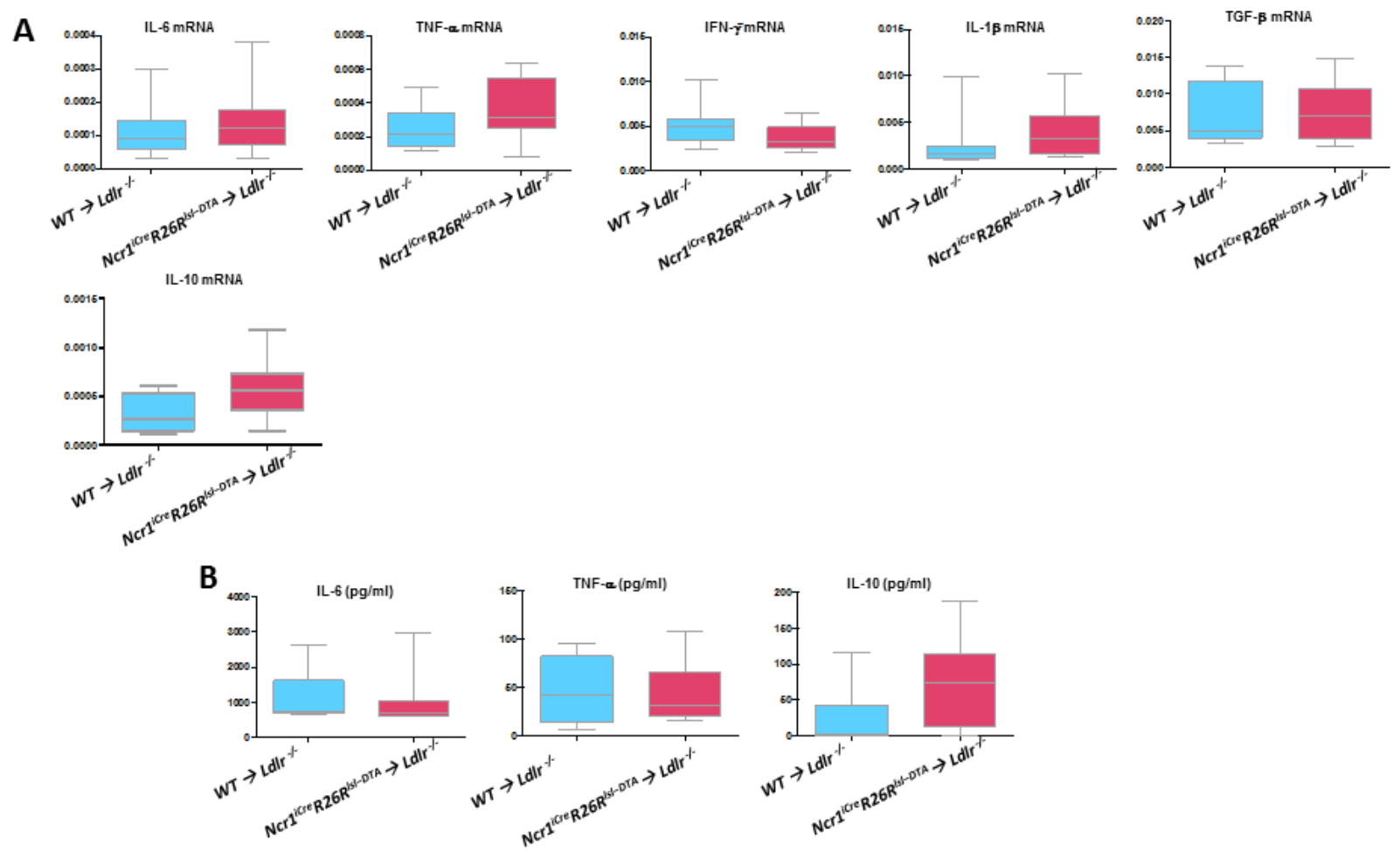

Supplementary Figure 3: (A) Quantification of IL-6, TNF- $\alpha$, IFN- $\gamma$, IL-1 $\beta$, IL-10, and TGF- $\beta$ mRNA expression in the spleens of chimeric $L d l r^{-/}$mice. (B) Cytokine production by lipopolysaccharide (LPS)/interferon (IFN)- $\gamma$-stimulated-splenocytes ( $24 \mathrm{hr}$ ) isolated from chimeric $\mathrm{Ldlr}^{-/}$mice. 


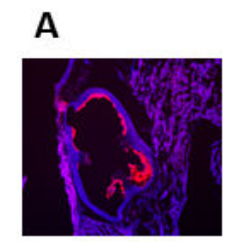

$W T \rightarrow$ Ldlr $^{-}$

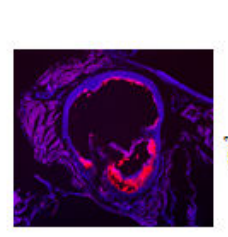

Noé $\rightarrow$ Ldlr $\%$

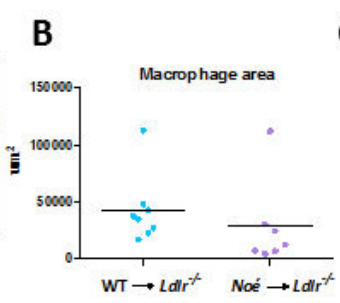

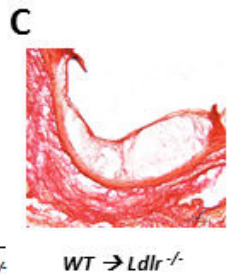

$W T \rightarrow L d l r \%$

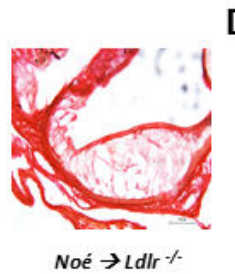

Noé $\rightarrow$ Ldll $\%$
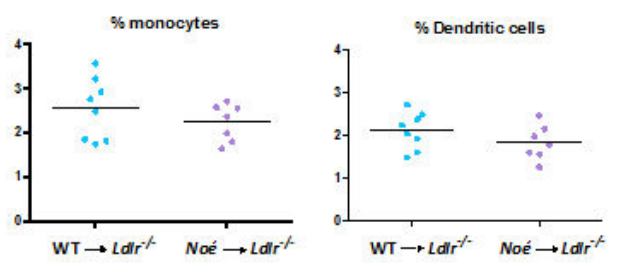

$\mathrm{F}$
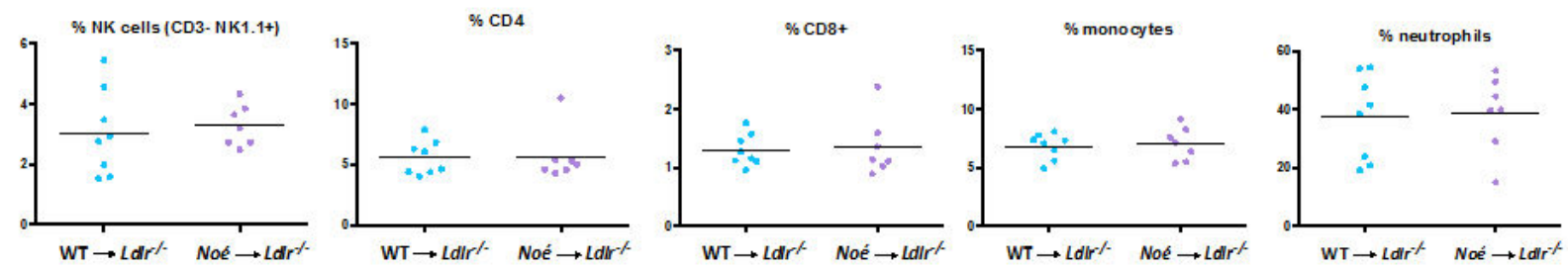

Supplementary Figure 4: Representative photomicrographs (A) of controls and Noé chimeric mice and quantitative analysis $(B)$ of macrophage accumulation (macrophage plus monocyte antibody, MOMA staining) in atherosclerotic lesions of chimeric $\mathrm{Ldlr}^{-/-}$mice. Representative photomicrographs (C) of controls and Noé chimeric mice and quantitative analysis (D) of lesion collagen content (Sirius red) of male chimeric $\mathrm{Ldlr}^{-/}$mice. Representative quantitative analysis of the percentage of lymphoid (CD4+, CD8+, NKT cells) and myeloid (monocytes, dendritic cells, neutrophils) populations in spleen (E) and blood (F) of control $(n=8)$ and Noé $(n=7)$ chimeric mice, 8 weeks HFD. 


\section{A}
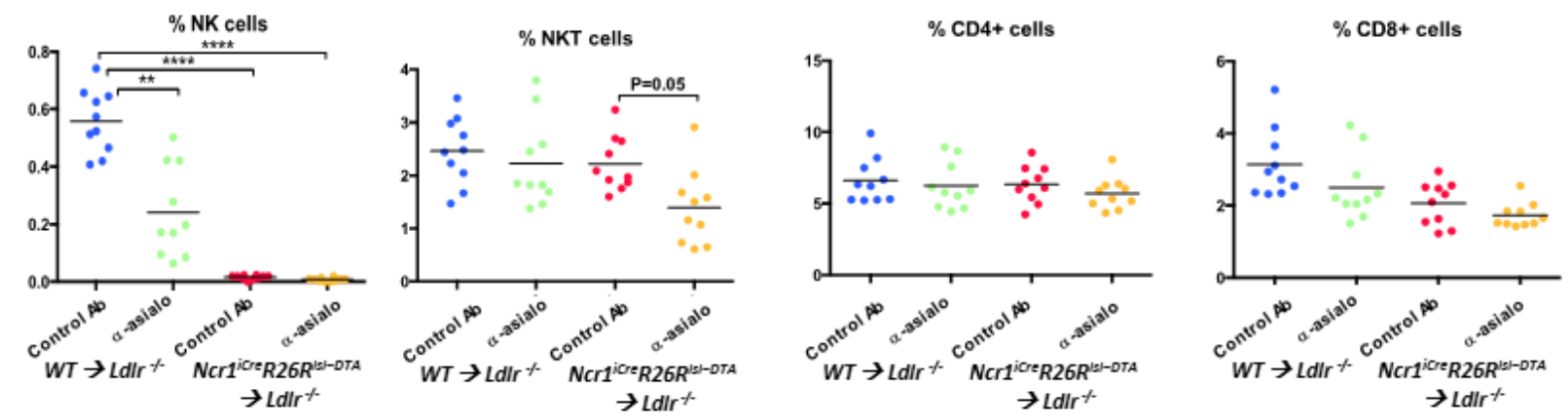

B
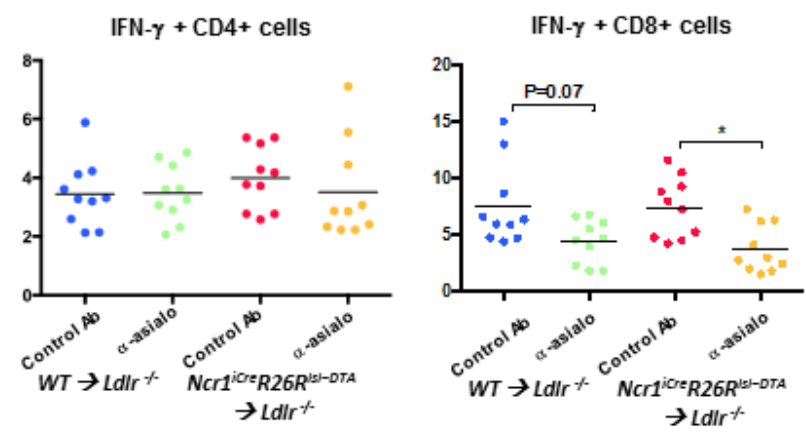

Supplementary Figure 5: (A) Representative quantitative analysis of spleen cells' percentage (NK, NKT, CD4, and CD8 cells) 5 days after commencing treatment with $\alpha$ -

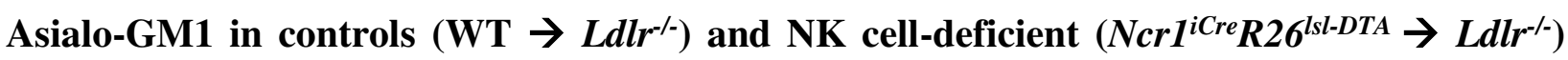
mice treated with either $\alpha$-Asialo-GM1 or control serum.

(B) Representative quantitative analysis of the percentage of IFN- $\gamma$-producing CD4 and CD8 $\mathrm{T}$ cells in the spleen of $\alpha$-Asialo-GM1- or control serum-treated controls (WT $\rightarrow \mathrm{Ldlr}^{-/-}$ ) and NK cell-deficient $\left(N c r I^{i C r e} R_{26}^{l s l-D T A} \rightarrow L^{L} d r^{--}\right)$mice. 
A

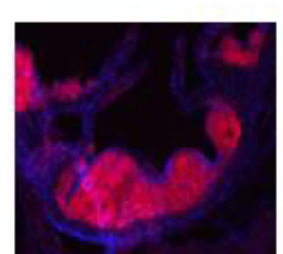

WT $\rightarrow$ Ldlr $\%$

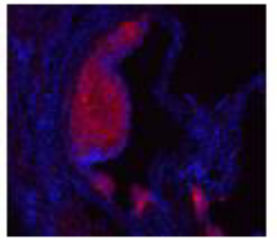

WT $\rightarrow$ Ldlr $\% \quad$ Ner1 $1^{i C r e} R 26 R^{i s l-D T A} \rightarrow L d l r \%$

+ poly $(l: C)$

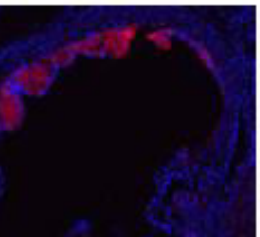

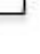

$$
+\operatorname{poly}(I: C)
$$

B

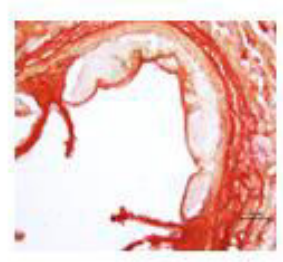

WT $\rightarrow$ Ldlr $\%$

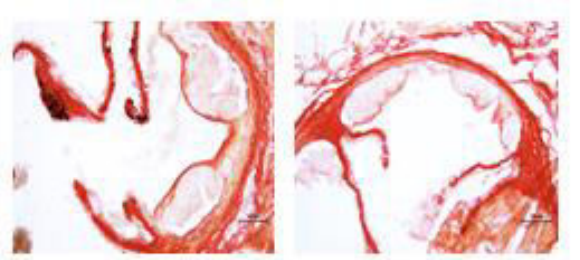

WT $\rightarrow$ Ldlr $\% \quad$ Ner $1^{\text {iCre } R 26 R^{\mid s j-D T A} \rightarrow L d l r} \%$

$+\operatorname{poly}(I: C)$
Macrophage content

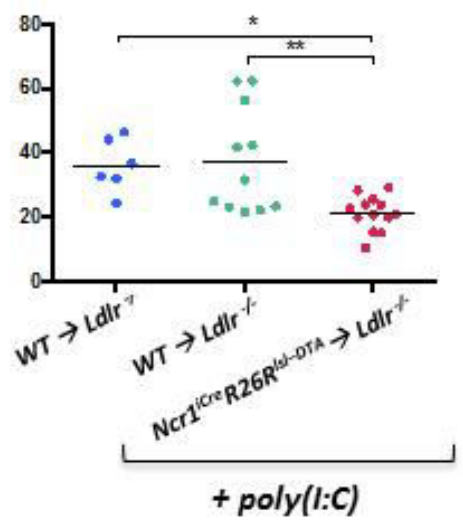

Collagen content

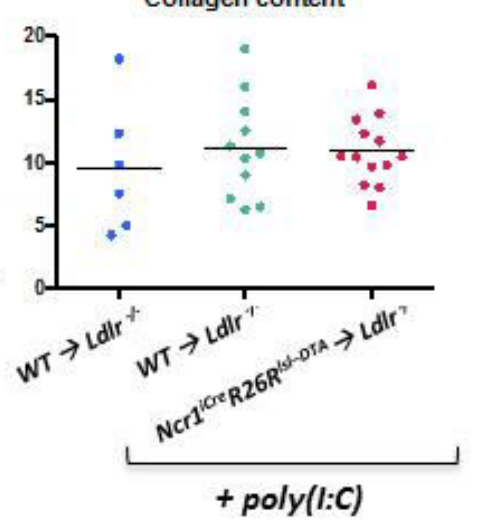

Supplementary Figure 6: Representative photomicrographs and quantitative analysis of macrophage accumulation (A) or collagen content $(B)$ in atherosclerotic lesions of saline- or

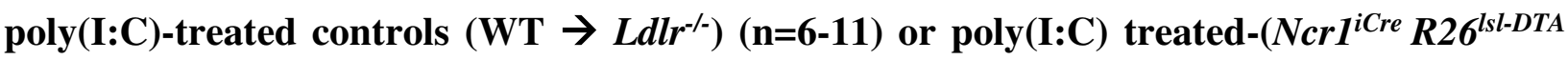
$\rightarrow$ Ldlr $\left.^{-1}\right)$ chimeric mice $(\mathrm{n}=13)$. 


\begin{tabular}{|c|c|c|c|c|c|c|c|c|c|c|c|c|c|c|c|}
\hline \multirow{6}{*}{$\begin{array}{l}\text { cholesterol } \\
(\mathrm{g} / \mathrm{L})\end{array}$} & \multicolumn{2}{|c|}{8 weeks } & \multicolumn{2}{|c|}{12 weeks } & \multicolumn{2}{|c|}{15 weeks } & \multicolumn{2}{|c|}{8 weeks } & \multicolumn{4}{|c|}{8 weeks } & \multicolumn{3}{|c|}{8 weeks } \\
\hline & WT & 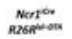 & WT & 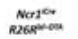 & WT & 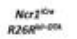 & WT & Noe & WT & WT & 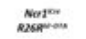 & Nenes & WT & WT & 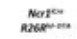 \\
\hline & $\downarrow$ & $!$ & $\downarrow$ & ! & $\downarrow$ & $!$ & $\downarrow$ & $\downarrow$ & $\downarrow$ & ! & $!$ & $\downarrow$ & $\downarrow$ & $\downarrow$ & $!$ \\
\hline & LDLr\% $/$ & LDLr $/$. & LDLr-/- & LDLr-/. & LDLr-/. & LDLr-/. & LDLr-/. & LDLr-/. & $\begin{array}{l}\text { LDLr-/ } \\
\text { (control } \\
\text { serum) }\end{array}$ & $\underset{\text { ( } \alpha \text {-asialo) }}{\text { LDLr } /-}$ & $\begin{array}{l}\text { LDLr-/- } \\
\text { (control } \\
\text { serum) }\end{array}$ & $\underset{(\alpha \text {-asialo) }}{\text { LDLr- } /-}$ & $\begin{array}{l}\text { LDLr-/- } \\
\text { (saline) }\end{array}$ & $\begin{array}{l}\text { LDLr-/- } \\
\text { (poly IC) }\end{array}$ & $\begin{array}{l}\text { LDLr-/- } \\
\text { (poly IC) }\end{array}$ \\
\hline & 8.4 & 7.4 & 10.6 & 9.9 & 10.4 & 11.3 & 10.4 & 9.8 & 9.3 & 8.7 & 9.8 & 9.1 & 10.3 & 9.4 & 11.2 \\
\hline & \pm 0.2 & \pm 0.3 & \pm 0.7 & \pm 1.1 & \pm 0.6 & \pm 1.1 & \pm 0.8 & \pm 1.4 & \pm 0.5 & \pm 0.6 & \pm 0.4 & \pm 0.5 & \pm 0.5 & \pm 0.7 & \pm 0.5 \\
\hline body & 26.3 & 27.5 & 28.7 & 33 & 30 & 30.7 & 28.9 & 28.5 & 26.5 & 27.1 & 26.7 & 26.4 & 28.7 & 26.8 & 26.5 \\
\hline weight (g) & \pm 0.5 & \pm 0.4 & \pm 0.7 & \pm 1.3 & \pm 0.9 & \pm 1.4 & \pm 0.4 & \pm 0.4 & \pm 0.3 & \pm 0.2 & \pm 0.4 & \pm 0.4 & \pm 0.5 & \pm 0.4 & \pm 0.3 \\
\hline
\end{tabular}

Table 1: Cholesterol levels and body weights in $\mathrm{Ldlr}^{-/-}$mice transplanted with bone marrow from WT, Ncr1 ${ }^{i C r e} R 26 R^{I s l-D T A}$ or Noé mice 


\section{Discussion}

Using two state-of-the-art genetic approaches, our study provides strong evidence that, in contrast to most previous studies, NK cells have no role in atherosclerosis development, unless a systemic inflammation is induced. Transfer of the BM of selectively depleted (Ncr $\left.1^{i C r e} R 26^{I s l-D T A}\right)$ or hyper-responsive (Noé) mice into lethally irradiated $L d l r^{--}$mice had no effect on plaque size (aortic root and thoracic aorta) and composition, or immune-inflammatory response, as compared with control mice. However, treating NK cell-deficient chimeric mice with Poly(I:C), in an attempt to mimic viral infection, significantly reduced lesion size, which suggests that NK cells might be proatherogenic only when activated by virus infection or inflammatory cytokines or in the context of tumor growth.

Earlier studies on the role of this innate immune cell population in atherosclerosis led to conflicting results. The very first studies to examine the role of NK cells in atherosclerosis used the Beige mutant mice. Accelerated atherosclerosis was observed in beige Ldlr-- mice and NK cells were accredited to be anti-atherogenic ${ }^{12}$. However, an alternative NK-cell independent hypothesis is most likely since the beige mutation which leads to functional changes in lysosome trafficking may involve other cells such as lesion macrophages or SMCs leading to increased foam cell formation or apoptosis ${ }^{33},{ }^{10}$. In contrast to these studies, $L d l r^{-/}$mice repopulated with Ly49A transgenic mice BM and which present a NK cell deficiency exhibited reduced atherosclerosis ${ }^{7}$. However, the expression of the inhibitory receptor Ly49A, which is regulated by granzyme A promoter in this model, is induced not only in NK cells, but also in subsets of $\mathrm{CD}^{+}$and $\mathrm{CD}^{+} \mathrm{T}$ cells ${ }^{16}$. Hence, this expression on $\mathrm{T}$ cells could be responsible for the decrease in lesion size in Ly49A transgenic BM recipients. Lately, experiments in Apoe ${ }^{-/}$mice fed a HFD treated with anti-asialo-GM1 antibody to deplete NK cells attenuated atherosclerosis. In addition, adoptive transfer of ex vivo activated NK cells increased lesion size in lymphocyte deficient Apoe $^{-/}$mice, an effect that was prevented by transferring perforin or granzymedeficient NK cells, with suggests that NK cells augment atherosclerosis via cytotoxic dependent mechanisms ${ }^{23}$. However, the expression of asialo-GM1 is not strictly confined to NK cells among hematopoietic cells and is detected in subpopulations of NKT, CD8 T, and $\gamma \delta$ T cells ${ }^{34},{ }^{35}$ and in some activated forms of $\mathrm{CD}^{+} \mathrm{T}$ cells, macrophages, eosinophils, and basophils under certain experimental conditions ${ }^{36},{ }^{37},{ }^{38}$. Our data suggest that the discrepancy observed in 
former studies was most likely due to effects on other cell populations targeted in addition to NK cells. Indeed, our study clearly demonstrates that NK cells have no role in atherosclerosis development.

NKp46 is also expressed in ILC1 and a subset of ILC3 (NKp46+ ILC3s). These cells are also deleted in the $N c r I^{i C r e} R 26 R^{D T A}$ mice ${ }^{25}$. In our experimental protocol, recipient mice were irradiated before BM transplantation. Both NK cells and ILC1s are sensitive to irradiation and should thus originate from donor BM. Regarding the ILC3 population, NKp46- ILC3 have been shown to be resistant to irradiation ${ }^{39},{ }^{40}$, but we have no information about the radioresistance of NKp46+ ILC3. Therefore, in our model of BM transplantation of $N c r 1^{i C r e} R 26 R^{D T A} \mathrm{BM}$ into irradiated $L d l r^{-/}$mice, not only NK cells but also ILC1s and potentially NKp46+ ILC3s should have been depleted, while other lymphocytes and myeloid cells were unaffected suggesting that these other ILCs subset does not play a major role in atherosclerosis either.

Moreover, we questioned the specificity of using anti-asialo GM1 treatment to target NK cells in the context of atherosclerosis. We clearly demonstrated its effect was NK cell-independent as atherosclerosis was reduced by anti-asialo-GM1 treatment to the same extent in control and NKdeficient mice. We observed that the anti-asialo-GM1 treatment not only depleted NK cells, but also strongly reduced $\mathrm{NKT}$ and $\mathrm{CD} 8^{+} \mathrm{T}$ cell population after 8 weeks of HFD. Among $\mathrm{CD} 8^{+} \mathrm{T}$ cells, the IFN- $\gamma$ - producing CD8 T cells was also reduced. This is consistent with a previous study showing that the asialo-GM1 ${ }^{+} \mathrm{CD} 8$ population are high IFN- $\gamma$ producing cells in response to stimulus ${ }^{34}$. These activated $\mathrm{CD}^{+} \mathrm{T}$ cells which are targeted by anti-asialo-GM1 antibodies might thus be involved in the phenotype observed in atherosclerosis. This is consistent with the fact that $\mathrm{CD}^{+} \mathrm{T}$ cell activation has been shown to exacerbate atherosclerosis ${ }^{41}$. Regarding the NKT cell population, although previous studies indicate that NK cell depletion with antibody against asialo-GM1 does not always drastically affect the size or function of NKT cell population ${ }^{42}$, NKT cell population was significantly decreased in hypercholesterolemic mice treated with anti-asialo-GM1 antibody. NKT cells are considered pro-atherogenic ${ }^{43}$. Their depletion could thus also contribute to the protective phenotype in anti-asialo-GM1-treated mice.

We used two models in which NK cells functions are increased: the Noé model in which NK cells are known to be intrinsically hyperresponsive and the Poly(I:C) model in which NK cells are activated due to an inflammatory environment.

Using the Noé mice, we showed that NK cell hyperresponsiveness in sterile conditions does not affect the atherosclerotic process, although hypercholesterolemia modestly increased IFN- $\gamma$ producing NK cell population. In contrast, a non-redundant role of NK cells was revealed in an 
inflammatory context induced by chronic Poly(I:C) injection. Along this line, previous studies reported an aggravation of atherosclerosis in hypercholesterolemic mice infected with mouse Cytomegalovirus (MCMV) ${ }^{44}$. NK cells are one of the first innate immune cells to respond to and contain the MCMV infection through their direct perforin mediated cytotoxicity function and IFN- $\gamma$ release ${ }^{45}$. In our study, the viral mimic Poly(I:C) significantly augmented NK cell activation marker CD69 as well as CD107a degranulation marker. CD107a is a lysosomal associated membrane protein lining the membrane of cytotoxic granules ${ }^{46}$. Its expression is correlated with degranulation and target cell lysis ${ }^{47}$. In addition, in our experiments, we noticed an upregulation of the mRNA of perforin, granzyme B, and IFN- $\gamma$ in the spleens of Poly(I:C)treated control mice. This highlights the marked activation of NK cells in response to Poly(I:C) and might account for the substantial reduction of atherosclerosis in Poly(I:C) treated NK celldeficient as compared to Poly(I:C) treated control mice. In control mice, we observed no effect of Poly(I:C) on atherosclerosis in the aortic root. The discrepancy observed could be attributed to the multiple effects of Poly(I:C) in atherosclerosis context. Zimmer et al. demonstrated that intravenous administration of Poly(I:C) induced endothelial dysfunction and increased atherosclerotic lesion development ${ }^{48}$. In contrast, Monaco's group described Poly(I:C)-mediated atheroprotection. Intraperitoneal administration of Poly(I:C) attenuated neointima formation and reduced injury induced media damage ${ }^{49}$. Further studies are required to fully elucidate how Poly(I:C) influence atherosclerosis. Nonetheless, this series of experiments clearly establish that even though resting NK cells have no effects on atherosclerosis, NK cells might exacerbate atherosclerosis in case of activation by an inflammatory context like viral infections. The ability of activated NK cells to influence other immune cells in the atherosclerotic process should be considered. In immune competent mice, NK cell-derived IFN- $\gamma$ promotes $\mathrm{CD} 4^{+} \mathrm{TH} 1$ priming ${ }^{50}$. It is well established that IFN- $\gamma$-producing TH1 subset is a major proatherogenic subset of the adaptive immune system and may activate pro-atherogenic properties of both vascular and immune cells ${ }^{51}$. Moreover, perforin and granzymes, which are highly augmented after Poly(I:C) treatment, lead to target cell apoptosis and can accelerate atherosclerosis by increasing necrosis 23 .

In conclusion, our findings indicate that NK cells have no role in atherosclerosis development in hypercholesterolemic mice, unless a super-imposed inflammatory status mimicking viral infection is induced. Further studies are needed for detailed characterization of the mechanisms whereby NK cells' response to viral infection crosslinks to atherosclerotic process. 


\section{Sources of Funding:}

This work was supported by Inserm, the Fondation pour la Recherche Medicale, CNRS (EV, SU), and Aix-Marseille University (EV, SU).

WNE has received a scholarship from the Association of Specialization and Scientific Guidance (ASSG).

Disclosures: none 


\section{References}

1. Le Borgne M, Caligiuri G, Nicoletti A. Once upon a time: The adaptive immune response in atherosclerosis--a fairy tale no more. Mol Med. 2015;21 Suppl 1:S13-18

2. Vanderlaan PA, Reardon CA. Thematic review series: The immune system and atherogenesis. The unusual suspects:An overview of the minor leukocyte populations in atherosclerosis. J Lipid Res. 2005;46:829-838

3. Trinchieri G. Biology of natural killer cells. Adv Immunol. 1989;47:187-376

4. Vivier E, Nunes JA, Vely F. Natural killer cell signaling pathways. Science. 2004;306:15171519

5. Vivier E, Raulet DH, Moretta A, Caligiuri MA, Zitvogel L, Lanier LL, Yokoyama WM, Ugolini S. Innate or adaptive immunity? The example of natural killer cells. Science. 2011;331:44-49

6. Bobryshev YV, Lord RS. Identification of natural killer cells in human atherosclerotic plaque. Atherosclerosis. 2005;180:423-427

7. Whitman SC, Rateri DL, Szilvassy SJ, Yokoyama W, Daugherty A. Depletion of natural killer cell function decreases atherosclerosis in low-density lipoprotein receptor null mice. Arterioscler Thromb Vasc Biol. 2004;24:1049-1054

8. Allavena P, Bianchi G, Zhou D, van Damme J, Jilek P, Sozzani S, Mantovani A. Induction of natural killer cell migration by monocyte chemotactic protein-1, -2 and -3. Eur J Immunol. $1994 ; 24: 3233-3236$

9. Umehara H, Bloom ET, Okazaki T, Nagano Y, Yoshie O, Imai T. Fractalkine in vascular biology: From basic research to clinical disease. Arterioscler Thromb Vasc Biol. 2004;24:34-40

10. Linton MF, Major AS, Fazio S. Proatherogenic role for nk cells revealed. Arterioscler Thromb Vasc Biol. 2004;24:992-994

11. Paigen B, Holmes PA, Novak EK, Swank RT. Analysis of atherosclerosis susceptibility in mice with genetic defects in platelet function. Arteriosclerosis. 1990;10:648-652

12. Schiller NK, Boisvert WA, Curtiss LK. Inflammation in atherosclerosis: Lesion formation in ldl receptor-deficient mice with perforin and lyst(beige) mutations. Arterioscler Thromb Vasc Biol. 2002;22:1341-1346 
13. Spritz RA. Genetic defects in chediak-higashi syndrome and the beige mouse. J Clin Immunol. 1998;18:97-105

14. Ward DM, Griffiths GM, Stinchcombe JC, Kaplan J. Analysis of the lysosomal storage disease chediak-higashi syndrome. Traffic. 2000;1:816-822

15. Ross R. The pathogenesis of atherosclerosis--an update. N Engl J Med. 1986;314:488-500

16. Kim S, Iizuka K, Aguila HL, Weissman IL, Yokoyama WM. In vivo natural killer cell activities revealed by natural killer cell-deficient mice. Proc Natl Acad Sci U S A. 2000;97:27312736

17. Radaev S, Sun PD. Structure and function of natural killer cell surface receptors. Annu Rev Biophys Biomol Struct. 2003;32:93-114

18. Kim S, Song YJ, Higuchi DA, Kang HP, Pratt JR, Yang L, Hong CM, Poursine-Laurent J, Iizuka K, French AR, Sunwoo JB, Ishii S, Reimold AM, Yokoyama WM. Arrested natural killer cell development associated with transgene insertion into the atf2 locus. Blood. 2006;107:10241030

19. To K, Agrotis A, Besra G, Bobik A, Toh BH. Nkt cell subsets mediate differential proatherogenic effects in apoe-/- mice. Arterioscler Thromb Vasc Biol. 2009;29:671-677

20. Oberg L, Eriksson M, Fahlen L, Sentman CL. Expression of ly49a on t cells alters the threshold for t cell responses. Eur J Immunol. 2000;30:2849-2856

21. Tupin E, Nicoletti A, Elhage R, Rudling M, Ljunggren HG, Hansson GK, Berne GP. Cd1ddependent activation of nkt cells aggravates atherosclerosis. $J$ Exp Med. 2004;199:417-422

22. Ludewig B, Freigang S, Jaggi M, Kurrer MO, Pei YC, Vlk L, Odermatt B, Zinkernagel RM, Hengartner H. Linking immune-mediated arterial inflammation and cholesterol-induced atherosclerosis in a transgenic mouse model. Proc Natl Acad Sci U S A. 2000;97:12752-12757

23. Selathurai A, Deswaerte V, Kanellakis P, Tipping P, Toh BH, Bobik A, Kyaw T. Natural killer (nk) cells augment atherosclerosis by cytotoxic-dependent mechanisms. Cardiovasc Res. 2014;102:128-137

24. Levy EM, Roberti MP, Mordoh J. Natural killer cells in human cancer: From biological functions to clinical applications. J Biomed Biotechnol. 2011;2011:676198

25. Deauvieau F, Fenis A, Dalencon F, Burdin N, Vivier E, Kerdiles Y. Lessons from nk cell deficiencies in the mouse. Curr Top Microbiol Immunol. 2016;395:173-190 
26. Narni-Mancinelli E, Jaeger BN, Bernat C, Fenis A, Kung S, De Gassart A, Mahmood S, Gut M, Heath SC, Estelle J, Bertosio E, Vely F, Gastinel LN, Beutler B, Malissen B, Malissen M, Gut IG, Vivier E, Ugolini S. Tuning of natural killer cell reactivity by nkp46 and helios calibrates t cell responses. Science. 2012;335:344-348

27. Narni-Mancinelli E, Chaix J, Fenis A, Kerdiles YM, Yessaad N, Reynders A, Gregoire C, Luche H, Ugolini S, Tomasello E, Walzer T, Vivier E. Fate mapping analysis of lymphoid cells expressing the nkp46 cell surface receptor. Proc Natl Acad Sci U S A. 2011;108:18324-18329

28. Joffre J, Potteaux S, Zeboudj L, Loyer X, Boufenzer A, Laurans L, Esposito B, Vandestienne M, de Jager SC, Henique C, Zlatanova I, Taleb S, Bruneval P, Tedgui A, Mallat Z, Gibot S, Ait-Oufella H. Genetic and pharmacological inhibition of trem-1 limits the development of experimental atherosclerosis. J Am Coll Cardiol. 2016;68:2776-2793

29. Mallat Z, Gojova A, Sauzeau V, Brun V, Silvestre JS, Esposito B, Merval R, Groux H, Loirand G, Tedgui A. Rho-associated protein kinase contributes to early atherosclerotic lesion formation in mice. Circ Res. 2003;93:884-888

30. Bustin SA, Benes V, Garson JA, Hellemans J, Huggett J, Kubista M, Mueller R, Nolan T, Pfaffl MW, Shipley GL, Vandesompele J, Wittwer CT. The miqe guidelines: Minimum information for publication of quantitative real-time pcr experiments. Clin Chem. 2009;55:611622

31. Vandesompele J, De Preter K, Pattyn F, Poppe B, Van Roy N, De Paepe A, Speleman F. Accurate normalization of real-time quantitative rt-pcr data by geometric averaging of multiple internal control genes. Genome Biol. 2002;3:Research0034

32. Vivier E, Ugolini S, Nunes JA. Adapted secretion of cytokines in nk cells. Nat Immunol. 2013;14:1108-1110

33. Glass CK, Witztum JL. Atherosclerosis. The road ahead. Cell. 2001;104:503-516

34. Lee U, Santa K, Habu S, Nishimura T. Murine asialo gm1+cd8+t cells as novel interleukin12-responsive killer t cell precursors. Jpn J Cancer Res. 1996;87:429-432

35. Trambley J, Bingaman AW, Lin A, Elwood ET, Waitze SY, Ha J, Durham MM, Corbascio M, Cowan SR, Pearson TC, Larsen CP. Asialo $\operatorname{gm} 1(+) \operatorname{cd} 8(+) \mathrm{t}$ cells play a critical role in costimulation blockade-resistant allograft rejection. J Clin Invest. 1999;104:1715-1722 
36. Wiltrout RH, Santoni A, Peterson ES, Knott DC, Overton WR, Herberman RB, Holden HT. Reactivity of anti-asialo gm1 serum with tumoricidal and non-tumoricidal mouse macrophages. $J$ Leukoc Biol. 1985;37:597-614

37. Kataoka S, Konishi Y, Nishio Y, Fujikawa-Adachi K, Tominaga A. Antitumor activity of eosinophils activated by il-5 and eotaxin against hepatocellular carcinoma. DNA Cell Biol. 2004;23:549-560

38. Nishikado H, Mukai K, Kawano Y, Minegishi Y, Karasuyama H. Nk cell-depleting antiasialo gm1 antibody exhibits a lethal off-target effect on basophils in vivo. J Immunol. 2011;186:5766-5771

39. Bando JK, Colonna M. Innate lymphoid cell function in the context of adaptive immunity. Nat Immunol. 2016;17:783-789

40. Dudakov JA, Hanash AM, Jenq RR, Young LF, Ghosh A, Singer NV, West ML, Smith OM, Holland AM, Tsai JJ, Boyd RL, van den Brink MR. Interleukin-22 drives endogenous thymic regeneration in mice. Science. 2012;336:91-95

41. Cochain C, Koch M, Chaudhari SM, Busch M, Pelisek J, Boon L, Zernecke A. Cd8+ t cells regulate monopoiesis and circulating ly6c-high monocyte levels in atherosclerosis in mice. Circ Res. 2015;117:244-253

42. Smyth MJ. Nk cells and nkt cells collaborate in host protection from methylcholanthreneinduced fibrosarcoma. Int Immunol. 2008;20:631

43. Getz GS, Reardon CA. Natural killer $\mathrm{t}$ cells in atherosclerosis. Nat Rev Cardiol. 2017;14:304-314

44. Vliegen I, Duijvestijn A, Grauls G, Herngreen S, Bruggeman C, Stassen F. Cytomegalovirus infection aggravates atherogenesis in apoe knockout mice by both local and systemic immune activation. Microbes Infect. 2004;6:17-24

45. Sumaria N, van Dommelen SL, Andoniou CE, Smyth MJ, Scalzo AA, Degli-Esposti MA. The roles of interferon-gamma and perforin in antiviral immunity in mice that differ in genetically determined nk-cell-mediated antiviral activity. Immunol Cell Biol. 2009;87:559-566

46. Winchester BG. Lysosomal membrane proteins. Eur J Paediatr Neurol. 2001;5 Suppl A:1119

47. Alter G, Malenfant JM, Altfeld M. Cd107a as a functional marker for the identification of natural killer cell activity. J Immunol Methods. 2004;294:15-22 
48. Zimmer S, Steinmetz M, Asdonk T, Motz I, Coch C, Hartmann E, Barchet W, Wassmann S, Hartmann G, Nickenig G. Activation of endothelial toll-like receptor 3 impairs endothelial function. Circ Res. 2011;108:1358-1366

49. Cole JE, Navin TJ, Cross AJ, Goddard ME, Alexopoulou L, Mitra AT, Davies AH, Flavell RA, Feldmann M, Monaco C. Unexpected protective role for toll-like receptor 3 in the arterial wall. Proc Natl Acad Sci U S A. 2011;108:2372-2377

50. Martin-Fontecha A, Thomsen LL, Brett S, Gerard C, Lipp M, Lanzavecchia A, Sallusto F. Induced recruitment of $\mathrm{nk}$ cells to lymph nodes provides ifn-gamma for $\mathrm{t}(\mathrm{h}) 1$ priming. Nat Immunol. 2004;5:1260-1265

51. Elhage R, Gourdy P, Brouchet L, Jawien J, Fouque MJ, Fievet C, Huc X, Barreira Y, Couloumiers JC, Arnal JF, Bayard F. Deleting tcr alpha beta+ or cd4+ t lymphocytes leads to opposite effects on site-specific atherosclerosis in female apolipoprotein e-deficient mice. Am $J$ Pathol. 2004;165:2013-2018 
III. RESULTS Part 2 


\section{Part 2: Analyzing NK Cell Localization in Mice and Human Plaques}

\section{Introduction}

NK cells have been reported in human and mouse atherosclerotic lesions. Earlier studies aimed at describing NK cell homing to the lesions were limited by the lack of specific antibody or the artificial milieu created for tracking. Firstly, Whitman and his colleagues used anti-Ly49G2 antibody, 4D11, to detect NK cells in the lesions of $\mathrm{Ldlr}^{-1}$ mice transplanted with the BM of either WT or Ly49A transgenic mice. However, 4D1 also stains NKT cells, which are DX5-positive T cells in addition to memory CD8 positive T lymphocytes ${ }^{1}$. In addition, in his study, Selathurai et al did adoptive transfer of NK cells isolated from congenic Ly5.1 mice into $\mathrm{Apoe}^{-/-} \mathrm{Rag}^{-{ }^{--}} \mathrm{IL} 2 \mathrm{rg}^{-/-}$mice 2 days before completing a HFD of 8 weeks. Then NK cells were stained against Ly5.1 and detected in close proximity to macrophages in atherosclerotic lesions ${ }^{2}$. However, the plausibility of this observation is limited by the fact that adoptively transferred NK cells do not represent physiological percentage of NK cells normally present in the mouse and its behavior when on a HFD. Here in, we describe a specific method of tracking NK cell homing to atherosclerotic plaques. We took advantage of the $N c r-1^{g f p / g f p}$ mice where only NK cells are green fluorescent protein (GFP)-positive ${ }^{3}$.

In addition, we collaborated with the platform of Anatomopathology at the European Hospital of Georges Pompidou (HEGP), where Patrick Bruneval stained the human carotid plaques with a newly developed antibody anti-NKp46, which specifically targets Human NK cells.

\section{Materials and Methods}

For tracking NK cells in mouse lesions, lethally irradiated $L d l r^{-/-}$mice were reconstituted with BM of $N c r-1^{g f p / g f p}$ mice and put on HFD for 8 weeks. The generation of $N c r-1^{g f p / g f p}$ mice was previously described by Ofer Mandelboim ${ }^{3}$. An internal ribosome entry site (IRES)-GFP fusion protein replaces the exons 5-7 of the Ncr-1 receptor-1, therefore all NK cells are GFP-positive. 
After 8 weeks HFD, frozen sections of aortic sinus of $N c r-1^{g f p / g f p} \rightarrow L d l r^{-/-}$mice were stained with rabbit anti-GFP antibody (NK cells) and counterstained with DAPI (nuclear dye) and MOMA-2 antibody (monocytes/macrophages). At least 4 sections per mouse were examined for immunostaining and the spleen was used as an internal control.

To detect NK cells in the human plaques, immunostaining studies were performed in atherosclerotic plaques from carotid arteries obtained post-mortem at autopsy. We used specific antibody against NKp46 protein in formalin fixed paraffin embedded tissues after antigen retrieval by heating in citrate buffer and $\mathrm{ABC}$ peroxidase technique as previously described ${ }^{4}$.

\section{Results}

\section{NK cells are scarcely detected in Human and Mouse Atherosclerotic Lesions}

To specifically track NK cell homing to atherosclerotic lesions in hypercholesterolemic mice, we reconstituted lethally irradiated $L d l r^{-/}$mice with the BM of $N c r-1$-GFP mice. After 8 weeks of HFD, staining atherosclerotic lesions with anti-GFP-antibody showed positive staining with the number of cells per lesion being relatively small, 1 to 2 cells per lesion examined (Fig. 1 A).

Immunohistochemical staining of human carotid arteries confirmed that few NK cells (anti-NKp46+) are found in early lesions. Only one or two cells were detected in the plaques of uncomplicated atherosclerosis, while none were observed in advanced plaque lesions (Fig. 1 B).

The spleen was used as an internal control in the two separate experiments. 
A

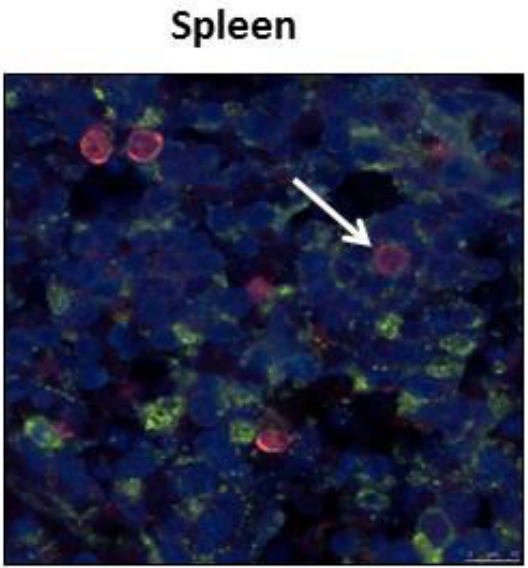

Spleen

B

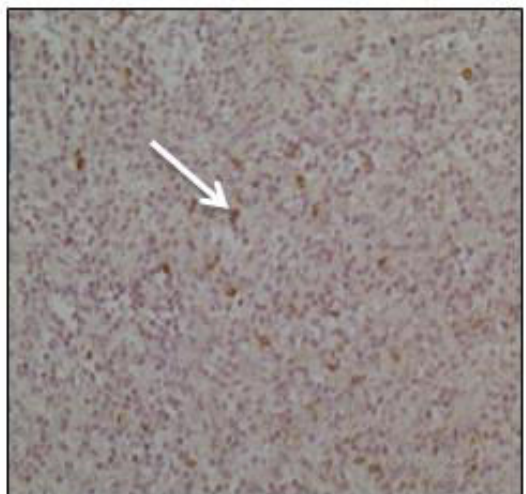

aortic sinus

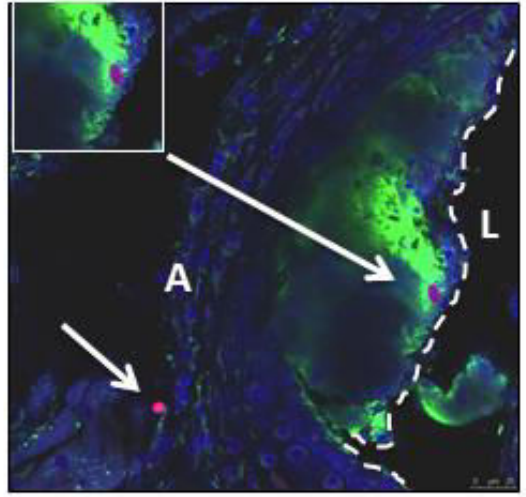

aortic sinus

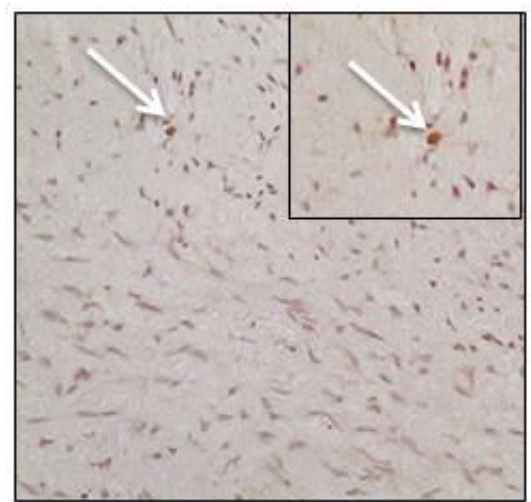

anti-MOMA-2

anti-GFP

DAPI

NKp46+

\section{Figure 1:}

(A) Photomicrographs of NK cells (anti-GFP+) detected in spleen and aortic sinus of Ncr-1 $1^{g f p / g f p} \rightarrow \mathrm{Ldll}^{-/}$mice at 8 weeks HFD, the dashed line marks the intima L: donates the lumen, $\mathrm{A}$ : donates the adventitia

(B) Immunohistochemistry in human atherosclerotic plaques. Few NKp46+ NK cells (brown) are detected in fatty streak lesions of the aorta but not in advanced carotid artery plaques 


\section{Discussion}

Due to the limitations of earlier studies used to determine NK cells in atherosclerotic lesions, here we describe a specific tracking method (Ncr-1 $1^{g f p / g f p}$ mice) to detect NK cell homing to the lesion. After 8 weeks of HFD, we were able to detect few NK cells in the intima and adventitia (one or two per lesion assessed).

In addition, we were able to detect only few NKp46+ NK cells in the human carotid plaques; one or two cells in fatty streak lesions but not in advanced lesions. This is congruent with an earlier study which states that NK cells represent a rare population of $0.1-0.5 \%$ of the total $\mathrm{T}$ cell population identified in atherosclerotic plaques ${ }^{5}$. Nonetheless, NK cell appearance, in addition to be of low frequency, does not reflect their involvement in the disease.

\section{References}

1. Whitman SC, Rateri DL, Szilvassy SJ, Yokoyama W, Daugherty A. Depletion of natural killer cell function decreases atherosclerosis in low-density lipoprotein receptor null mice. Arterioscler Thromb Vasc Biol. 2004;24:10491054

2. Selathurai A, Deswaerte V, Kanellakis P, Tipping P, Toh BH, Bobik A, Kyaw T. Natural killer (nk) cells augment atherosclerosis by cytotoxic-dependent mechanisms. Cardiovasc Res. 2014;102:128-137

3. Gazit R, Gruda R, Elboim M, Arnon TI, Katz G, Achdout H, Hanna J, Qimron U, Landau G, Greenbaum E, Zakay-Rones Z, Porgador A, Mandelboim O. Lethal influenza infection in the absence of the natural killer cell receptor gene ncr1. Nat Immunol. 2006;7:517-523

4. Bariety J, Mandet C, Hill GS, Bruneval P. Parietal podocytes in normal human glomeruli. J Am Soc Nephrol. 2006;17:2770-2780

5. Bobryshev YV, Lord RS. Identification of natural killer cells in human atherosclerotic plaque. Atherosclerosis. 2005;180:423-427 
IV. GENERAL DISCUSSION and

CONCLUSION 
Cardiovascular diseases have become the most frequent cause of death globally [389]. An estimated 17.3 million die from CVD each year, equivalent to $30 \%$ of all deaths worldwide. Atherosclerosis, which affects large and medium sized arteries, is the underlying cause of most CVDs[390]. Today, the traditional view of atherosclerosis as resulting from passive build up of cholesterol in the artery wall is no more available. Accumulating evidence implicates inflammation in the pathogenesis of atherosclerosis, where effector cells of the innate and adaptive immunity are involved at all stages of the disease [76].

Numerous studies identified the role of different subsets of macrophages, T cells, B cells, and NKT cells, however, the role of NK cells is still unclear. The limitation of earlier studies assessing the role of NK cells in atherosclerosis has been discussed in details in Chapter III.

My findings provide definitive evidence of almost no role played by NK cells in atherosclerosis development, which contrasts with previous studies. I used two state-of-theart genetic approaches, of NK cell depletion $\left(N c r l^{i C r e} R 26 R^{l s l-D T A}\right)$ or hyper-activation (Noé). Then, I transferred the bone marrow of these mice into lethally irradiated $L d L r^{-/}$mice. The chimeric mice showed no change in plaque size, composition, or immune inflammatory response in comparison with control mice. In a third approach, I demonstrated that adding an additional external inflammatory response (poly I:C) where NK cells are activated would augment atherosclerosis.

In the first part of my thesis, I used a genetically modified model of NK cell depletion; $N c r I^{i C r e} R 26 R^{l s l-D T A}$ mice. I have shown that NK cell depletion does not affect plaque size and composition or the immune inflammatory response. NKp46 receptor is expressed by NK cells at all stages of their development, in addition to a tiny population of ILCs, ILC1 and NKp46+ILC3s [349]. So these cells are also deleted in $N c r 1^{i C r e} R 26 R^{l s l-D T A}$ mice. However, we know that a subset of ILC3 population is resistant to irradiation, so this population is present in our bone marrow transplantation experiments. For the moment, we know nothing about how these tiny populations could contribute to atherosclerosis. But we can suggest they have no role either.

In the second part of our study, I demonstrated that even hyperresponsive NK cells (Noé mice) do not affect atherosclerosis development. The Noé baseline mice are more resistant to viral infections due to the higher frequency of IFN- $\gamma$-producing and CD107-expressing NK 
cells. Although the chimeric mice $\left(N o e ́-L d l r^{-/}\right)$had higher frequency of IFN- $\gamma+\mathrm{NK}$ cells, the mice exhibited no change regarding the size or composition of the plaque. Hence, we can conclude that hypercholesterolemia alone is not an enough stimulus for NK cells to intervene in the atherosclerotic process.

In the third part of the thesis, I reported the non-selectivity of the anti-asialo-GM1 treatment at least in atherosclerosis context. Anti-asialo-GM1 treatment reduced atherosclerosis to the same extent in NK-deficient and control mice. So, we concluded that anti-asialo-GM1 effect is NK cell independent, but rather mediated by other cell populations. Interestingly, in addition to efficiently depleting NK cells, anti-asialo GM1 also had a more or less comparable effect on CD8 T and NKT populations. Specifically, IFN- $\gamma$ CD8 cells were importantly reduced. Since NKT and CD8 are proatherognic, we suggested that the observed effects on atherosclerosis could be mediated by these populations.

Finally, we clearly demonstrated that NK cells overactivated in an additional inflammatory context could exacerbate atherosclerosis development. Poly (I:C) (a viral mimic) significantly increased perforin, granzyme $\mathrm{B}$, and IFN- $\gamma$ expression, as well as the frequency of CD107a, CD69, and IFN- $\gamma$ NK cells. This was associated with significant reduction in atherosclerosis in Poly (I:C)-treated NK cell deficient mice compared to controls. Perforin and granzymes, which are highly augmented after poly I:C treatment, lead to target cell apoptosis and can accelerate atherosclerosis by increasing necrosis [374]. It has been demonstrated previously that activated CD8 $+\mathrm{T}$ cells accelerate atherosclerosis by perforin/granzyme dependent mechanisms [391]. This could be a common mechanism between activated NK cells and CD8+ T cells.

Additional indirect mechanisms may also contribute to activated-NK cells' atherogenecity. It is now well appreciated that NK cells facilitate differentiation of naïve T cells into TH1 T cells by providing an early source of IFN- $\gamma$ in the lymphnodes, which is required for TH1 polarization, as mentioned earlier. So, we suggest that NK cells might also promote atherosclerosis via CD4+ T cell dependent mechanism.

Moreover, we observed decreased macrophage infiltration in poly (I:C)-treated NK deficient mice which suggests that NK cells might be implicated in their recruitment to the developing lesions. Macrophages in atherosclerotic lesions express MICA-B, a ligand of NK cell 
activating receptor NKG2D [392]. The binding of ligandsto NKG2D receptors activates NK cells and promotes cytotoxicity of ligand-expressing cells.

Apart from this, I used a specific tracking method ( $N c r-1^{g f p / g f p}$ mice) to detect NK cell homing to the lesion. After 8 weeks of HFD, we were able to detect few NK cells in the intima and adventitia (one or two per lesion assessed). In addition, we were able to detect only few NKp46+ NK cells in the human carotid plaques; one or two cells in fatty streak lesions but not in advanced lesions. This is congruent with an earlier study where NK cells were detected in human plaques by staining for CD56+CD3- cells. In that study, the authors stated that NK cells represent a rare population of $0.1-0.5 \%$ of the total $\mathrm{T}$ cell population identified in the same sections [381]. Nonetheless, NK cell appearance, in addition to be of low frequency, does not reflect their involvement in the disease. NK cells are highly migratory, expressing a large number of chemokine receptors including CXCR1, CXCR3, CXCR7, CCR7, and CCR9 [393]. MCP-1 and fractalkine (CX3CL1), which are dominantly augmented in atherosclerosis, are the most relevant cytokines, driving NK cell migration [394]. So their presence in the lesions could be only a result of an altered chemokine and cytokine spectrum.

In conclusion, our work clearly demonstrates that NK cells have no role in atherosclerosis development in hypercholesterolemic mice, unless activated by an additional inflammatory stimulus. Although NK cells are present, albeit at low frequency in early lesions, their depletion as well as hyperresponsiveness does not change the disease evolution. NK cells, activated by an inflammatory context, would enhance atherosclerosis and therefore might represent a target for therapeutic intervention in virus-induced atherosclerosis exacerbation. (Figure 17) 


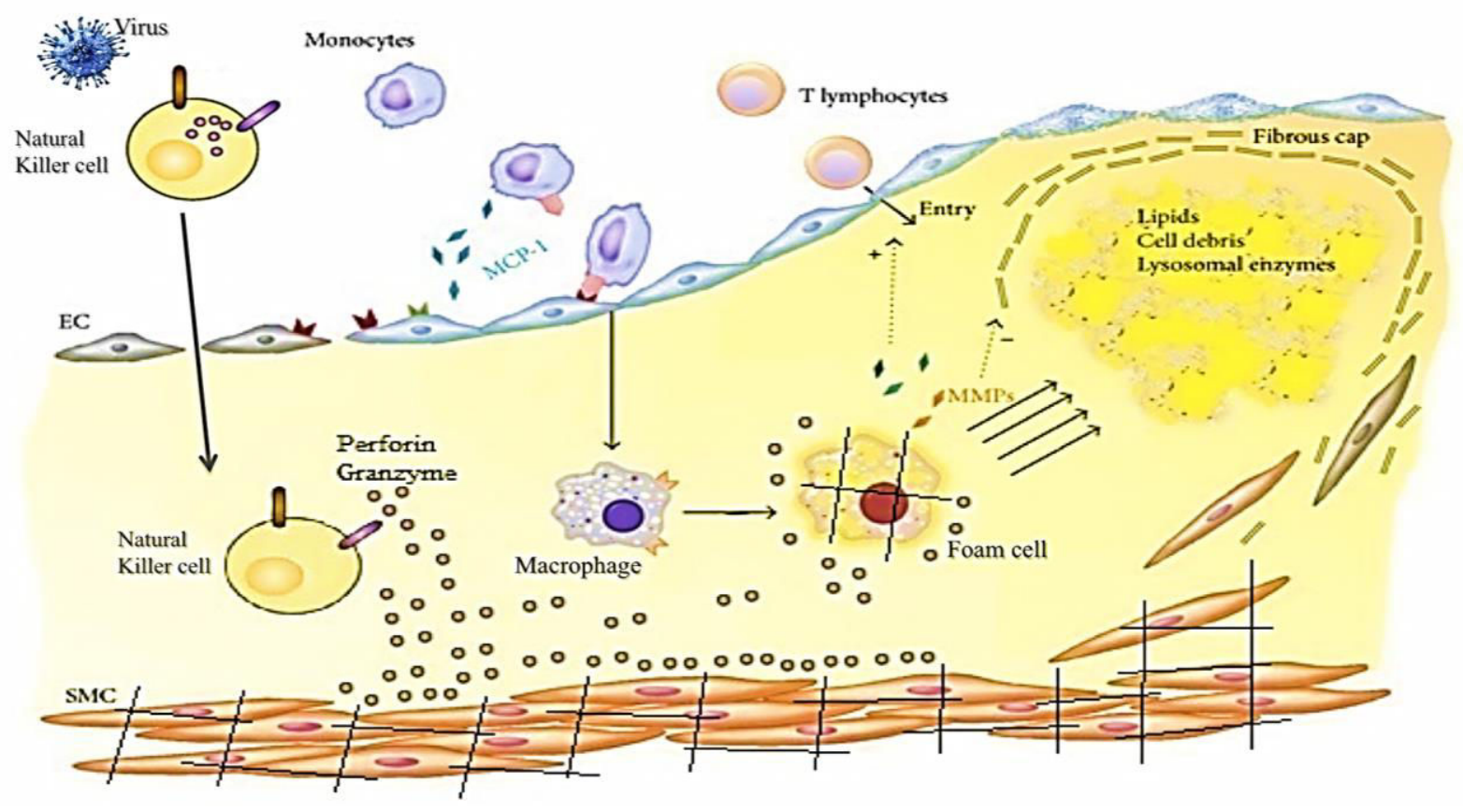

Figure 17: Proposed mechanism of NK cell-enhanced atherogenicity in an inflammatory context. 
V. BIBLIOGRAPAY 
1. Tedgui, A. and Z. Mallat, Cytokines in atherosclerosis: pathogenic and regulatory pathways. Physiol Rev, 2006. 86(2): p. 515-81.

2. Ruffer, M.A., On arterial lesions found in Egyptian mummies (1580 B.C.-525 A.D.). The Journal of Pathology and Bacteriology, 1911. 15(4): p. 453-462.

3. Le Borgne, M., G. Caligiuri, and A. Nicoletti, Once Upon a Time: The Adaptive Immune Response in Atherosclerosis--a Fairy Tale No More. Mol Med, 2015. 21 Suppl 1: p. S13-8.

4. Ross, R. and L. Harker, Hyperlipidemia and atherosclerosis. Science, 1976. 193(4258): p. 1094-100.

5. Fishbein, G.A. and M.C. Fishbein, Arteriosclerosis: rethinking the current classification. Arch Pathol Lab Med, 2009. 133(8): p. 1309-16.

6. Classics in arteriosclerosis research: On experimental cholesterin steatosis and its significance in the origin of some pathological processes by N. Anitschkow and S. Chalatow, translated by Mary Z. Pelias, 1913. Arteriosclerosis, 1983. 3(2): p. 17882.

7. Ross, R. and J.A. Glomset, The pathogenesis of atherosclerosis (first of two parts). N Engl J Med, 1976. 295(7): p. 369-77.

8. Ross, R., Atherosclerosis--an inflammatory disease. N Engl J Med, 1999. 340(2): p. 115-26.

9. Jonasson, L., et al., Regional accumulations of $T$ cells, macrophages, and smooth muscle cells in the human atherosclerotic plaque. Arteriosclerosis, 1986. 6(2): p. 131-8.

10. Dahlof, B., Cardiovascular disease risk factors: epidemiology and risk assessment. Am J Cardiol, 2010. 105(1 Suppl): p. 3a-9a.

11. Tolfrey, K., Intraindividual variability of children's blood lipid and lipoprotein concentrations: a review. Prev Cardiol, 2002. 5(3): p. 145-51.

12. Falk, E., Pathogenesis of atherosclerosis. J Am Coll Cardiol, 2006. 47(8 Suppl): p. C7-12.

13. Third Report of the National Cholesterol Education Program (NCEP) Expert Panel on Detection, Evaluation, and Treatment of High Blood Cholesterol in Adults (Adult Treatment Panel III) final report. Circulation, 2002. 106(25): p. 3143-421.

14. Hebert, P.R., et al., Cholesterol lowering with statin drugs, risk of stroke, and total mortality. An overview of randomized trials. Jama, 1997. 278(4): p. 313-21.

15. Mazzolai, L., et al., Endogenous angiotensin II induces atherosclerotic plaque vulnerability and elicits a Th1 response in ApoE-/- mice. Hypertension, 2004. 44(3): p. 277-82.

16. Tedgui, A. and Z. Mallat, Hypertension: a novel regulator of adaptive immunity in atherosclerosis? Hypertension, 2004. 44(3): p. 257-8.

17. Executive Summary of The Third Report of The National Cholesterol Education Program (NCEP) Expert Panel on Detection, Evaluation, And Treatment of High Blood Cholesterol In Adults (Adult Treatment Panel III). Jama, 2001. 285(19): p. 2486-97.

18. Blake, G.J. and P.M. Ridker, Novel clinical markers of vascular wall inflammation. Circ Res, 2001. 89(9): p. 763-71.

19. Ridker, P.M. and D.A. Morrow, C-reactive protein, inflammation, and coronary risk. Cardiol Clin, 2003. 21(3): p. 315-25.

20. Sakkinen, P.A., et al., Clustering of procoagulation, inflammation, and fibrinolysis variables with metabolic factors in insulin resistance syndrome. Am J Epidemiol, 2000. 152(10): p. 897-907. 
21. Winkler, G., et al., Elevated serum TNF-alpha level as a link between endothelial dysfunction and insulin resistance in normotensive obese patients. Diabet Med, 1999. 16(3): p. 207-11.

22. Singhal, A., et al., Influence of leptin on arterial distensibility: a novel link between obesity and cardiovascular disease? Circulation, 2002. 106(15): p. 1919-24.

23. Uemura, S., et al., Diabetes mellitus enhances vascular matrix metalloproteinase activity: role of oxidative stress. Circ Res, 2001. 88(12): p. 1291-8.

24. Danesh, J., R. Collins, and R. Peto, Chronic infections and coronary heart disease: is there a link? Lancet, 1997. 350(9075): p. 430-6.

25. del Rincon, I.D., et al., High incidence of cardiovascular events in a rheumatoid arthritis cohort not explained by traditional cardiac risk factors. Arthritis Rheum, 2001. 44(12): p. 2737-45.

26. Sherer, Y. and Y. Shoenfeld, Mechanisms of disease: atherosclerosis in autoimmune diseases. Nat Clin Pract Rheumatol, 2006. 2(2): p. 99-106.

27. Gonzalez-Gay, M.A., et al., Endothelial dysfunction, carotid intima-media thickness, and accelerated atherosclerosis in rheumatoid arthritis. Semin Arthritis Rheum, 2008. 38(2): p. 67-70.

28. Wilhelm, A.J., et al., Dysregulated CD4+ T cells from SLE-susceptible mice are sufficient to accelerate atherosclerosis in LDLr-/- mice. Ann Rheum Dis, 2015. 74(4): p. 778-85.

29. Chatzizisis, Y.S., et al., Role of endothelial shear stress in the natural history of coronary atherosclerosis and vascular remodeling: molecular, cellular, and vascular behavior. J Am Coll Cardiol, 2007. 49(25): p. 2379-93.

30. Libby, P., Inflammation in atherosclerosis. Nature, 2002. 420(6917): p. 868-74.

31. Asakura, T. and T. Karino, Flow patterns and spatial distribution of atherosclerotic lesions in human coronary arteries. Circ Res, 1990. 66(4): p. 1045-66.

32. $\mathrm{Ku}, \mathrm{D} . \mathrm{N} .$, et al., Pulsatile flow and atherosclerosis in the human carotid bifurcation. Positive correlation between plaque location and low oscillating shear stress. Arteriosclerosis, 1985. 5(3): p. 293-302.

33. Wentzel, J.J., et al., Endothelial shear stress in the evolution of coronary atherosclerotic plaque and vascular remodelling: current understanding and remaining questions. Cardiovasc Res, 2012. 96(2): p. 234-43.

34. Millonig, G., et al., Network of vascular-associated dendritic cells in intima of healthy young individuals. Arterioscler Thromb Vasc Biol, 2001. 21(4): p. 503-8.

35. Dewey, C.F., Jr., et al., The dynamic response of vascular endothelial cells to fluid shear stress. J Biomech Eng, 1981. 103(3): p. 177-85.

36. VanderLaan, P.A., C.A. Reardon, and G.S. Getz, Site specificity of atherosclerosis: site-selective responses to atherosclerotic modulators. Arterioscler Thromb Vasc Biol, 2004. 24(1): p. 12-22.

37. Chappell, D.C., et al., Oscillatory shear stress stimulates adhesion molecule expression in cultured human endothelium. Circ Res, 1998. 82(5): p. 532-9.

38. Peng, H.B., P. Libby, and J.K. Liao, Induction and stabilization of I kappa B alpha by nitric oxide mediates inhibition of NF-kappa B. J Biol Chem, 1995. 270(23): p. 14214-9.

39. Uematsu, M., et al., Regulation of endothelial cell nitric oxide synthase $m R N A$ expression by shear stress. Am J Physiol, 1995. 269(6 Pt 1): p. C1371-8.

40. Shaul, P.W., Endothelial nitric oxide synthase, caveolae and the development of atherosclerosis. J Physiol, 2003. 547(Pt 1): p. 21-33. 
41. Stary, H.C., et al., A definition of initial, fatty streak, and intermediate lesions of atherosclerosis. A report from the Committee on Vascular Lesions of the Council on Arteriosclerosis, American Heart Association. Circulation, 1994. 89(5): p. 2462-78.

42. Stary, H.C., et al., A definition of advanced types of atherosclerotic lesions and a histological classification of atherosclerosis. A report from the Committee on Vascular Lesions of the Council on Arteriosclerosis, American Heart Association. Circulation, 1995. 92(5): p. 1355-74.

43. Virmani, R., et al., Lessons from sudden coronary death: a comprehensive morphological classification scheme for atherosclerotic lesions. Arterioscler Thromb Vasc Biol, 2000. 20(5): p. 1262-75.

44. Bentzon, J.F., et al., Mechanisms of plaque formation and rupture. Circ Res, 2014. 114(12): p. 1852-66.

45. Velican, D. and C. Velican, Atherosclerotic involvement of the coronary arteries of adolescents and young adults. Atherosclerosis, 1980. 36(4): p. 449-60.

46. Schwartz, S.M., D. deBlois, and E.R. O'Brien, The intima. Soil for atherosclerosis and restenosis. Circ Res, 1995. 77(3): p. 445-65.

47. Burke, A.P., et al., Coronary risk factors and plaque morphology in men with coronary disease who died suddenly. N Engl J Med, 1997. 336(18): p. 1276-82.

48. Davies, M.J., Stability and instability: two faces of coronary atherosclerosis. The Paul Dudley White Lecture 1995. Circulation, 1996. 94(8): p. 2013-20.

49. Burke, A.P., et al., Effect of risk factors on the mechanism of acute thrombosis and sudden coronary death in women. Circulation, 1998. 97(21): p. 2110-6.

50. van der Wal, A.C., et al., Site of intimal rupture or erosion of thrombosed coronary atherosclerotic plaques is characterized by an inflammatory process irrespective of the dominant plaque morphology. Circulation, 1994. 89(1): p. 36-44.

51. Farb, A., et al., Coronary plaque erosion without rupture into a lipid core. A frequent cause of coronary thrombosis in sudden coronary death. Circulation, 1996. 93(7): p. 1354-63.

52. Durand, E., et al., In vivo induction of endothelial apoptosis leads to vessel thrombosis and endothelial denudation: a clue to the understanding of the mechanisms of thrombotic plaque erosion. Circulation, 2004. 109(21): p. 2503-6.

53. Kragel, A.H., et al., Morphometric analysis of the composition of atherosclerotic plaques in the four major epicardial coronary arteries in acute myocardial infarction and in sudden coronary death. Circulation, 1989. 80(6): p. 1747-56.

54. Constantinides, P., Plaque fissures in human coronary thrombosis. Journal of Atherosclerosis Research. 6(1): p. 1-17.

55. Glagov, S., et al., Compensatory enlargement of human atherosclerotic coronary arteries. N Engl J Med, 1987. 316(22): p. 1371-5.

56. Paigen, B., et al., Variation in susceptibility to atherosclerosis among inbred strains of mice. Atherosclerosis, 1985. 57(1): p. 65-73.

57. Shi, W., et al., Determinants of atherosclerosis susceptibility in the C3H and C57BL/6 mouse model: evidence for involvement of endothelial cells but not blood cells or cholesterol metabolism. Circ Res, 2000. 86(10): p. 1078-84.

58. Kashyap, V.S., et al., Apolipoprotein E deficiency in mice: gene replacement and prevention of atherosclerosis using adenovirus vectors. J Clin Invest, 1995. 96(3): p. 1612-20.

59. Tenger, C. and X. Zhou, Apolipoprotein E modulates immune activation by acting on the antigen-presenting cell. Immunology, 2003. 109(3): p. 392-7. 
60. Meir, K.S. and E. Leitersdorf, Atherosclerosis in the apolipoprotein-E-deficient mouse: a decade of progress. Arterioscler Thromb Vasc Biol, 2004. 24(6): p. 100614.

61. Piedrahita, J.A., et al., Generation of mice carrying a mutant apolipoprotein E gene inactivated by gene targeting in embryonic stem cells. Proc Natl Acad Sci U S A, 1992. 89(10): p. 4471-5.

62. Getz, G.S. and C.A. Reardon, Do the Apoe-/- and Ldlr-/- Mice Yield the Same Insight on Atherogenesis? Arterioscler Thromb Vasc Biol, 2016. 36(9): p. 1734-41.

63. Nakashima, Y., et al., ApoE-deficient mice develop lesions of all phases of atherosclerosis throughout the arterial tree. Arterioscler Thromb, 1994. 14(1): p. 133-40.

64. Reddick, R.L., S.H. Zhang, and N. Maeda, Atherosclerosis in mice lacking apo E. Evaluation of lesional development and progression. Arterioscler Thromb, 1994. 14(1): p. 141-7.

65. Daugherty, A., et al., Recommendation on Design, Execution, and Reporting of Animal Atherosclerosis Studies: A Scientific Statement From the American Heart Association. Circ Res, 2017.

66. Defesche, J.C., Low-density lipoprotein receptor--its structure, function, and mutations. Semin Vasc Med, 2004. 4(1): p. 5-11.

67. Marais, A.D., Familial hypercholesterolaemia. Clin Biochem Rev, 2004. 25(1): p. 49-68.

68. Ishibashi, S., et al., Hypercholesterolemia in low density lipoprotein receptor knockout mice and its reversal by adenovirus-mediated gene delivery. J Clin Invest, 1993. 92(2): p. 883-93.

69. Knowles, J.W. and N. Maeda, Genetic modifiers of atherosclerosis in mice. Arterioscler Thromb Vasc Biol, 2000. 20(11): p. 2336-45.

70. Hartvigsen, K., et al., A diet-induced hypercholesterolemic murine model to study atherogenesis without obesity and metabolic syndrome. Arterioscler Thromb Vasc Biol, 2007. 27(4): p. 878-85.

71. Getz, G.S. and C.A. Reardon, Animal models of atherosclerosis. Arterioscler Thromb Vasc Biol, 2012. 32(5): p. 1104-15.

72. Hobbs, H.H., et al., The LDL receptor locus in familial hypercholesterolemia: mutational analysis of a membrane protein. Annu Rev Genet, 1990. 24: p. 133-70.

73. Lee, Y.T., et al., Mouse models of atherosclerosis: a historical perspective and recent advances. 2017. 16(1): p. 12.

74. Emini Veseli, B., et al., Animal models of atherosclerosis. Eur J Pharmacol, 2017.

75. Stary, H.C., et al., A definition of the intima of human arteries and of its atherosclerosis-prone regions. A report from the Committee on Vascular Lesions of the Council on Arteriosclerosis, American Heart Association. Arterioscler Thromb, 1992. 12(1): p. 120-34.

76. Libby, P., P.M. Ridker, and G.K. Hansson, Progress and challenges in translating the biology of atherosclerosis. Nature, 2011. 473(7347): p. 317-25.

77. Han, C.I., G.R. Campbell, and J.H. Campbell, Circulating bone marrow cells can contribute to neointimal formation. J Vasc Res, 2001. 38(2): p. 113-9.

78. Bennett, M.R., S. Sinha, and G.K. Owens, Vascular Smooth Muscle Cells in Atherosclerosis. Circ Res, 2016. 118(4): p. 692-702.

79. Wolinsky, H. and S. Glagov, A lamellar unit of aortic medial structure and function in mammals. Circ Res, 1967. 20(1): p. 99-111. 
80. Roselaar, S.E., P.X. Kakkanathu, and A. Daugherty, Lymphocyte populations in atherosclerotic lesions of apoE -/- and LDL receptor -/- mice. Decreasing density with disease progression. Arterioscler Thromb Vasc Biol, 1996. 16(8): p. 1013-8.

81. Ross, R., J. Glomset, and L. Harker, Response to injury and atherogenesis. Am J Pathol, 1977. 86(3): p. 675-84.

82. Williams, K.J. and I. Tabas, The response-to-retention hypothesis of early atherogenesis. Arterioscler Thromb Vasc Biol, 1995. 15(5): p. 551-61.

83. Berliner, J., et al., Oxidized lipids in atherogenesis: formation, destruction and action. Thromb Haemost, 1997. 78(1): p. 195-9.

84. Williams, K.J. and I. Tabas, The response-to-retention hypothesis of atherogenesis reinforced. Curr Opin Lipidol, 1998. 9(5): p. 471-4.

85. Glass, C.K. and J.L. Witztum, Atherosclerosis. the road ahead. Cell, 2001. 104(4): p. 503-16.

86. Poole, J.C. and H.W. Florey, Changes in the endothelium of the aorta and the behaviour of macrophages in experimental atheroma of rabbits. J Pathol Bacteriol, 1958. 75(2): p. 245-51.

87. Libby, P., P.M. Ridker, and A. Maseri, Inflammation and atherosclerosis. Circulation, 2002. 105(9): p. 1135-43.

88. Wilensky, R.L. and D. Hamamdzic, The molecular basis of vulnerable plaque: potential therapeutic role for immunomodulation. Curr Opin Cardiol, 2007. 22(6): p. 545-51.

89. Mestas, J. and K. Ley, Monocyte-endothelial cell interactions in the development of atherosclerosis. Trends Cardiovasc Med, 2008. 18(6): p. 228-32.

90. Koenen, R.R. and C. Weber, Chemokines: established and novel targets in atherosclerosis. EMBO Mol Med, 2011. 3(12): p. 713-25.

91. Libby, P., Changing concepts of atherogenesis. J Intern Med, 2000. 247(3): p. 34958.

92. Goldstein, J.L., et al., Binding site on macrophages that mediates uptake and degradation of acetylated low density lipoprotein, producing massive cholesterol deposition. Proc Natl Acad Sci U S A, 1979. 76(1): p. 333-7.

93. Steinbrecher, U.P., et al., Modification of low density lipoprotein by endothelial cells involves lipid peroxidation and degradation of low density lipoprotein phospholipids. Proc Natl Acad Sci U S A, 1984. 81(12): p. 3883-7.

94. Chellan, B., et al., Enzymatically Modified Low-Density Lipoprotein Promotes Foam Cell Formation in Smooth Muscle Cells via Macropinocytosis and Enhances Receptor-Mediated Uptake of Oxidized Low-Density Lipoprotein. Arterioscler Thromb Vasc Biol, 2016. 36(6): p. 1101-13.

95. Hansson, G.K. and A. Hermansson, The immune system in atherosclerosis. Nat Immunol, 2011. 12(3): p. 204-12.

96. Woollard, K.J. and F. Geissmann, Monocytes in atherosclerosis: subsets and functions. Nat Rev Cardiol, 2010. 7(2): p. 77-86.

97. Napoli, C., et al., Fatty streak formation occurs in human fetal aortas and is greatly enhanced by maternal hypercholesterolemia. Intimal accumulation of low density lipoprotein and its oxidation precede monocyte recruitment into early atherosclerotic lesions. J Clin Invest, 1997. 100(11): p. 2680-90.

98. Ylitalo, R., et al., Effects of clodronate (dichloromethylene bisphosphonate) on the development of experimental atherosclerosis in rabbits. J Lab Clin Med, 1994. 123(5): p. 769-76. 
99. Smith, J.D., et al., Decreased atherosclerosis in mice deficient in both macrophage colony-stimulating factor (op) and apolipoprotein E. Proceedings of the National Academy of Sciences of the United States of America, 1995. 92(18): p. 8264-8268.

100. Woollard, K.J., Immunological aspects of atherosclerosis. Clin Sci (Lond), 2013. 125(5): p. 221-35.

101. Swirski, F.K., et al., Ly-6Chi monocytes dominate hypercholesterolemia-associated monocytosis and give rise to macrophages in atheromata. J Clin Invest, 2007. 117(1): p. 195-205.

102. Tacke, F., et al., Monocyte subsets differentially employ CCR2, CCR5, and CX3CR1 to accumulate within atherosclerotic plaques. J Clin Invest, 2007. 117(1): p. 185-94.

103. Yvan-Charvet, L., et al., ATP-binding cassette transporters and HDL suppress hematopoietic stem cell proliferation. Science, 2010. 328(5986): p. 1689-93.

104. Combadiere, C., et al., Combined inhibition of CCL2, CX3CR1, and CCR5 abrogates Ly6C(hi) and Ly6C(lo) monocytosis and almost abolishes atherosclerosis in hypercholesterolemic mice. Circulation, 2008. 117(13): p. 1649-57.

105. Robbins, C.S., et al., Extramedullary hematopoiesis generates Ly-6C(high) monocytes that infiltrate atherosclerotic lesions. Circulation, 2012. 125(2): p. 36474.

106. Libby, P., A.H. Lichtman, and G.K. Hansson, Immune effector mechanisms implicated in atherosclerosis: from mice to humans. Immunity, 2013. 38(6): p. 1092-104.

107. Moore, K.J., F.J. Sheedy, and E.A. Fisher, Macrophages in atherosclerosis: a dynamic balance. Nat Rev Immunol, 2013. 13(10): p. 709-21.

108. Kuchibhotla, S., et al., Absence of CD36 protects against atherosclerosis in ApoE knock-out mice with no additional protection provided by absence of scavenger receptor A I/II. Cardiovasc Res, 2008. 78(1): p. 185-96.

109. Manning-Tobin, J.J., et al., Loss of SR-A and CD36 activity reduces atherosclerotic lesion complexity without abrogating foam cell formation in hyperlipidemic mice. Arterioscler Thromb Vasc Biol, 2009. 29(1): p. 19-26.

110. Tabas, I., Consequences and therapeutic implications of macrophage apoptosis in atherosclerosis: the importance of lesion stage and phagocytic efficiency. Arterioscler Thromb Vasc Biol, 2005. 25(11): p. 2255-64.

111. Gui, T., et al., Diverse roles of macrophages in atherosclerosis: from inflammatory biology to biomarker discovery. Mediators Inflamm, 2012. 2012: p. 693083.

112. Autieri, M.V., Pro- and Anti-Inflammatory Cytokine Networks in Atherosclerosis. ISRN Vascular Medicine, 2012. 2012: p. 17.

113. Randolph, G.J., J. Ochando, and S. Partida-Sanchez, Migration of dendritic cell subsets and their precursors. Annu Rev Immunol, 2008. 26: p. 293-316.

114. Buono, C. and A.H. Lichtman, Co-stimulation and plaque-antigen-specific T-cell responses in atherosclerosis. Trends Cardiovasc Med, 2004. 14(4): p. 166-72.

115. Geissmann, F., et al., Development of monocytes, macrophages, and dendritic cells. Science, 2010. 327(5966): p. 656-61.

116. Galkina, E. and K. Ley, Immune and inflammatory mechanisms of atherosclerosis (*). Annu Rev Immunol, 2009. 27: p. 165-97.

117. Erbel, C., et al., Functional profile of activated dendritic cells in unstable atherosclerotic plaque. Basic Res Cardiol, 2007. 102(2): p. 123-32.

118. Bobryshev, Y.V., et al., Evidence that dendritic cells infiltrate atherosclerotic lesions in apolipoprotein E-deficient mice. Histol Histopathol, 2001. 16(3): p. 801-8. 
119. Niessner, A., et al., Pathogen-sensing plasmacytoid dendritic cells stimulate cytotoxic T-cell function in the atherosclerotic plaque through interferon-alpha. Circulation, 2006. 114(23): p. 2482-9.

120. Niessner, A. and C.M. Weyand, Dendritic Cells in Atherosclerotic Disease. Clinical immunology (Orlando, Fla.), 2010. 134(1): p. 25.

121. Paulson, K.E., et al., Resident intimal dendritic cells accumulate lipid and contribute to the initiation of atherosclerosis. Circ Res, 2010. 106(2): p. 383-90.

122. Gautier, E.L., et al., Conventional dendritic cells at the crossroads between immunity and cholesterol homeostasis in atherosclerosis. Circulation, 2009. 119(17): p. 2367-75.

123. Sage, A.P., et al., MHC Class II-restricted antigen presentation by plasmacytoid dendritic cells drives proatherogenic T cell immunity. Circulation, 2014. 130(16): p. 1363-73.

124. Daissormont, I.T., et al., Plasmacytoid dendritic cells protect against atherosclerosis by tuning T-cell proliferation and activity. Circ Res, 2011. 109(12): p. 1387-95.

125. Bot, I., et al., Perivascular mast cells promote atherogenesis and induce plaque destabilization in apolipoprotein E-deficient mice. Circulation, 2007. 115(19): p. 2516-25.

126. Libby, P., History of Discovery: Inflammation in Atherosclerosis. Arteriosclerosis, thrombosis, and vascular biology, 2012. 32(9): p. 2045-2051.

127. Hansson, G.K., A.K. Robertson, and C. Soderberg-Naucler, Inflammation and atherosclerosis. Annu Rev Pathol, 2006. 1: p. 297-329.

128. Hansson, G.K. and A. Hermansson, The immune system in atherosclerosis. Nat Immunol, 2011. 12(3): p. 204-212.

129. Stemme, S., J. Holm, and G.K. Hansson, T lymphocytes in human atherosclerotic plaques are memory cells expressing CD45RO and the integrin VLA-1. Arterioscler Thromb, 1992. 12(2): p. 206-11.

130. Zhou, X., et al., Transfer of $C D 4(+) T$ cells aggravates atherosclerosis in immunodeficient apolipoprotein E knockout mice. Circulation, 2000. 102(24): p. 2919-22.

131. Huber, S.A., et al., T helper-cell phenotype regulates atherosclerosis in mice under conditions of mild hypercholesterolemia. Circulation, 2001. 103(21): p. 2610-6.

132. Robertson, A.K. and G.K. Hansson, T cells in atherogenesis: for better or for worse? Arterioscler Thromb Vasc Biol, 2006. 26(11): p. 2421-32.

133. Ponnuswamy, P., et al., Humoral and cellular immune responses in atherosclerosis: spotlight on B-and T-cells. Vascul Pharmacol, 2012. 56(5-6): p. 193-203.

134. Gupta, S., et al., IFN-gamma potentiates atherosclerosis in ApoE knock-out mice. J Clin Invest, 1997. 99(11): p. 2752-61.

135. Buono, C., et al., Influence of interferon-gamma on the extent and phenotype of dietinduced atherosclerosis in the LDLR-deficient mouse. Arterioscler Thromb Vasc Biol, 2003. 23(3): p. 454-60.

136. Elhage, R., et al., Reduced atherosclerosis in interleukin-18 deficient apolipoprotein E-knockout mice. Cardiovasc Res, 2003. 59(1): p. 234-40.

137. Davenport, P. and P.G. Tipping, The role of interleukin-4 and interleukin-12 in the progression of atherosclerosis in apolipoprotein E-deficient mice. Am J Pathol, 2003. 163(3): p. 1117-25.

138. Hauer, A.D., et al., Blockade of interleukin-12 function by protein vaccination attenuates atherosclerosis. Circulation, 2005. 112(7): p. 1054-62. 
139. Whitman, S.C., P. Ravisankar, and A. Daugherty, Interleukin-18 enhances atherosclerosis in apolipoprotein E(-/-) mice through release of interferon-gamma. Circ Res, 2002. 90(2): p. E34-8.

140. Baidya, S.G. and Q.T. Zeng, Helper T cells and atherosclerosis: the cytokine web. Postgrad Med J, 2005. 81(962): p. 746-52.

141. Buono, C., et al., T-bet deficiency reduces atherosclerosis and alters plaque antigenspecific immune responses. Proc Natl Acad Sci U S A, 2005. 102(5): p. 1596-601.

142. Frostegard, J., et al., Cytokine expression in advanced human atherosclerotic plaques: dominance of pro-inflammatory (Th1) and macrophage-stimulating cytokines. Atherosclerosis, 1999. 145(1): p. 33-43.

143. Taleb, S., A. Tedgui, and Z. Mallat, Interleukin-17: friend or foe in atherosclerosis? Curr Opin Lipidol, 2010. 21(5): p. 404-8.

144. Ng, H.P., R.L. Burris, and S. Nagarajan, Attenuated atherosclerotic lesions in apoEFcgamma-chain-deficient hyperlipidemic mouse model is associated with inhibition of Th17 cells and promotion of regulatory T cells. J Immunol, 2011. 187(11): p. 6082-93.

145. Taleb, S., et al., Loss of SOCS3 expression in T cells reveals a regulatory role for interleukin-17 in atherosclerosis. J Exp Med, 2009. 206(10): p. 2067-77.

146. Ait-Oufella, H., et al., B cell depletion reduces the development of atherosclerosis in mice. J Exp Med, 2010. 207(8): p. 1579-87.

147. Taleb, S., A. Tedgui, and Z. Mallat, Regulatory T-cell immunity and its relevance to atherosclerosis. J Intern Med, 2008. 263(5): p. 489-99.

148. de Boer, O.J., et al., Low Numbers of FOXP3 Positive Regulatory T Cells Are Present in all Developmental Stages of Human Atherosclerotic Lesions. PLoS ONE, 2007. 2(8): p. e779.

149. Mor, A., et al., Altered status of CD4(+)CD25(+) regulatory T cells in patients with acute coronary syndromes. Eur Heart J, 2006. 27(21): p. 2530-7.

150. Mor, A., et al., Role of naturally occurring CD4+CD25+ regulatory $T$ cells in experimental atherosclerosis. Arterioscler Thromb Vasc Biol, 2007. 27(4): p. 893900.

151. Mallat, Z., et al., Inhibition of transforming growth factor-beta signaling accelerates atherosclerosis and induces an unstable plaque phenotype in mice. Circ Res, 2001. 89(10): p. 930-4.

152. Mallat, Z., H. Ait-Oufella, and A. Tedgui, Regulatory T-cell immunity in atherosclerosis. Trends Cardiovasc Med, 2007. 17(4): p. 113-8.

153. Potteaux, S., et al., Leukocyte-derived interleukin 10 is required for protection against atherosclerosis in low-density lipoprotein receptor knockout mice. Arterioscler Thromb Vasc Biol, 2004. 24(8): p. 1474-8.

154. Ait-Oufella, $\mathrm{H}_{\text {., }}$ et al., Natural regulatory $T$ cells control the development of atherosclerosis in mice. Nat Med, 2006. 12(2): p. 178-80.

155. Gotsman, I., et al., Impaired regulatory T-cell response and enhanced atherosclerosis in the absence of inducible costimulatory molecule. Circulation, 2006. 114(19): p. 2047-55.

156. Maganto-Garcia, E., et al., Foxp3+-inducible regulatory $T$ cells suppress endothelial activation and leukocyte recruitment. J Immunol, 2011. 187(7): p. 3521-9.

157. Lin, J., et al., The role of $C D 4+C D 25+$ regulatory $T$ cells in macrophage-derived foam-cell formation. J Lipid Res, 2010. 51(5): p. 1208-17. 
158. Olofsson, P.S., et al., CD137 is expressed in human atherosclerosis and promotes development of plaque inflammation in hypercholesterolemic mice. Circulation, 2008. 117(10): p. 1292-301.

159. Ludewig, B., et al., Linking immune-mediated arterial inflammation and cholesterol-induced atherosclerosis in a transgenic mouse model. Proc Natl Acad Sci U S A, 2000. 97(23): p. 12752-7.

160. Gotsman, I., et al., Proatherogenic immune responses are regulated by the PD1/PD-L pathway in mice. J Clin Invest, 2007. 117(10): p. 2974-82.

161. Jonasson, L., A. Tompa, and A. Wikby, Expansion of peripheral CD8+ T cells in patients with coronary artery disease: relation to cytomegalovirus infection. J Intern Med, 2003. 254(5): p. 472-8.

162. Bendelac, A., et al., CD1 Recognition by Mouse NK1 $\$ \wedge+\$ T$ Lymphocytes. Science, 1995. 268(5212): p. 863-865.

163. Tan, J.-q., et al., Type I natural killer T cells: naturally born for fighting. Acta Pharmacologica Sinica, 2010. 31(9): p. 1123-1132.

164. Ostos, M.A., et al., Implication of natural killer $T$ cells in atherosclerosis development during a LPS-induced chronic inflammation. FEBS Lett, 2002. 519(13): p. 23-9.

165. Major, A.S., et al., Quantitative and qualitative differences in proatherogenic NKT cells in apolipoprotein E-deficient mice. Arterioscler Thromb Vasc Biol, 2004. 24(12): p. 2351-7.

166. Nakai, Y., et al., Natural killer T cells accelerate atherogenesis in mice. Blood, 2004. 104(7): p. 2051-9.

167. Tupin, E., et al., CD1d-dependent Activation of NKT Cells Aggravates Atherosclerosis. The Journal of Experimental Medicine, 2004. 199(3): p. 417-422.

168. VanderLaan, P.A., et al., Characterization of the natural killer T-cell response in an adoptive transfer model of atherosclerosis. Am J Pathol, 2007. 170(3): p. 1100-7.

169. Caligiuri, G., et al., Protective immunity against atherosclerosis carried by $B$ cells of hypercholesterolemic mice. The Journal of Clinical Investigation, 2002. 109(6): p. 745-753.

170. Major, A.S., S. Fazio, and M.F. Linton, B-lymphocyte deficiency increases atherosclerosis in LDL receptor-null mice. Arterioscler Thromb Vasc Biol, 2002. 22(11): p. 1892-8.

171. Binder, C.J., et al., IL-5 links adaptive and natural immunity specific for epitopes of oxidized LDL and protects from atherosclerosis. J Clin Invest, 2004. 114(3): p. 42737.

172. Nilsson, J., G.K. Hansson, and P.K. Shah, Immunomodulation of atherosclerosis: implications for vaccine development. Arterioscler Thromb Vasc Biol, 2005. 25(1): p. 18-28.

173. Kyaw, T., et al., Conventional B2 B cell depletion ameliorates whereas its adoptive transfer aggravates atherosclerosis. J Immunol, 2010. 185(7): p. 4410-9.

174. Kiessling, R., E. Klein, and H. Wigzell, "Natural" killer cells in the mouse. I. Cytotoxic cells with specificity for mouse Moloney leukemia cells. Specificity and distribution according to genotype. Eur J Immunol, 1975. 5(2): p. 112-7.

175. Vivier, E., What is natural in natural killer cells? Immunol Lett, 2006. 107(1): p. 17.

176. Lanier, L.L., et al., Natural killer cells: definition of a cell type rather than a function. J Immunol, 1986. 137(9): p. 2735-9. 
177. Herberman, R.B. and J.R. Ortaldo, Natural killer cells: their roles in defenses against disease. Science, 1981. 214(4516): p. 24-30.

178. Di Santo, J.P., Natural killer cell developmental pathways: a question of balance. Annu Rev Immunol, 2006. 24: p. 257-86.

179. Vivier, E., J. Di Santo, and A. Moretta, Natural Killer Cells. 2016: Springer International Publishing.

180. Sun, J.C. and L.L. Lanier, NK cell development, homeostasis and function: parallels with CD8(+) T cells. Nat Rev Immunol, 2011. 11(10): p. 645-57.

181. Vivier, E., et al., Innate or adaptive immunity? The example of natural killer cells. Science, 2011. 331(6013): p. 44-9.

182. Orr, M.T. and L.L. Lanier, Natural killer cell education and tolerance. Cell, 2010. 142(6): p. 847-56.

183. Biron, C.A., K.S. Byron, and J.L. Sullivan, Severe herpesvirus infections in an adolescent without natural killer cells. N Engl J Med, 1989. 320(26): p. 1731-5.

184. Koch, J., et al., Activating natural cytotoxicity receptors of natural killer cells in cancer and infection. Trends Immunol, 2013. 34(4): p. 182-91.

185. Yokoyama, W.M., S. Kim, and A.R. French, The dynamic life of natural killer cells. Annu Rev Immunol, 2004. 22: p. 405-29.

186. Huntington, N.D., C.A. Vosshenrich, and J.P. Di Santo, Developmental pathways that generate natural-killer-cell diversity in mice and humans. Nat Rev Immunol, 2007. 7(9): p. 703-14.

187. Yu, J., A.G. Freud, and M.A. Caligiuri, Location and cellular stages of natural killer cell development. Trends Immunol, 2013. 34(12): p. 573-82.

188. Pinhas, N., et al., Murine peripheral NK-cell populations originate from site-specific immature NK cells more than from BM-derived NK cells. Eur J Immunol, 2016. 46(5): p. 1258-70.

189. Vosshenrich, C.A., et al., A thymic pathway of mouse natural killer cell development characterized by expression of GATA-3 and CD127. Nat Immunol, 2006. 7(11): p. 1217-24.

190. Kumar, V., et al., Natural killer cells in mice treated with 89strontium: normal target-binding cell numbers but inability to kill even after interferon administration. J Immunol, 1979. 123(4): p. 1832-8.

191. Seaman, W.E., et al., beta-Estradiol reduces natural killer cells in mice. J Immunol, 1978. 121(6): p. 2193-8.

192. Freud, A.G. and M.A. Caligiuri, Human natural killer cell development. Immunol Rev, 2006. 214: p. 56-72.

193. Iizuka, K., et al., Requirement for membrane lymphotoxin in natural killer cell development. Proc Natl Acad Sci U S A, 1999. 96(11): p. 6336-40.

194. Wu, Q., et al., Signal via lymphotoxin-beta $R$ on bone marrow stromal cells is required for an early checkpoint of NK cell development. J Immunol, 2001. 166(3): p. 1684-9.

195. Kondo, M., I.L. Weissman, and K. Akashi, Identification of clonogenic common lymphoid progenitors in mouse bone marrow. Cell, 1997. 91(5): p. 661-72.

196. Wang, B., et al., A block in both early $T$ lymphocyte and natural killer cell development in transgenic mice with high-copy numbers of the human CD3E gene. Proc Natl Acad Sci U S A, 1994. 91(20): p. 9402-6.

197. Flamand, V., et al., Delayed maturation of CD4- CD8- FC gamma RII/III+ T and natural killer cell precursors in Fc epsilon RI gamma transgenic mice. J Exp Med, 1996. 184(5): p. 1725-35. 
198. Suzuki, H., et al., Abnormal development of intestinal intraepithelial lymphocytes and peripheral natural killer cells in mice lacking the IL-2 receptor beta chain. J Exp Med, 1997. 185(3): p. 499-505.

199. Kennedy, M.K., et al., Reversible defects in natural killer and memory CD8 $T$ cell lineages in interleukin 15-deficient mice. J Exp Med, 2000. 191(5): p. 771-80.

200. Lodolce, J.P., et al., IL-15 receptor maintains lymphoid homeostasis by supporting lymphocyte homing and proliferation. Immunity, 1998. 9(5): p. 669-76.

201. Yokota, Y., et al., Development of peripheral lymphoid organs and natural killer cells depends on the helix-loop-helix inhibitor Id2. Nature, 1999. 397(6721): p. 702-6.

202. Barton, K., et al., The Ets-1 transcription factor is required for the development of natural killer cells in mice. Immunity, 1998. 9(4): p. 555-63.

203. Kim, S., et al., In vivo developmental stages in murine natural killer cell maturation. Nat Immunol, 2002. 3(6): p. 523-8.

204. Dokun, A.O., et al., Specific and nonspecific NK cell activation during virus infection. Nat Immunol, 2001. 2(10): p. 951-6.

205. Narni-Mancinelli, E., et al., Fate mapping analysis of lymphoid cells expressing the NKp46 cell surface receptor. Proc Natl Acad Sci U S A, 2011. 108(45): p. 18324-9.

206. Hayakawa, Y. and M.J. Smyth, CD27 dissects mature NK cells into two subsets with distinct responsiveness and migratory capacity. J Immunol, 2006. 176(3): p. 151724.

207. Hayakawa, Y., et al., Functional subsets of mouse natural killer cells. Immunol Rev, 2006. 214: p. 47-55.

208. Chiossone, L., et al., Maturation of mouse NK cells is a 4-stage developmental program. Blood, 2009. 113(22): p. 5488-96.

209. Caligiuri, M.A., Human natural killer cells. Blood, 2008. 112(3): p. 461-9.

210. Lanier, L.L., et al., The relationship of CD16 (Leu-11) and Leu-19 (NKH-1) antigen expression on human peripheral blood NK cells and cytotoxic T lymphocytes. J Immunol, 1986. 136(12): p. 4480-6.

211. Bennett, I.M., et al., Definition of a natural killer NKR-P1A+/CD56-/CD16functionally immature human $N K$ cell subset that differentiates in vitro in the presence of interleukin 12. J Exp Med, 1996. 184(5): p. 1845-56.

212. Wang, L.L., et al., Inducible expression of the gp49B inhibitory receptor on NK cells. J Immunol, 2000. 164(10): p. 5215-20.

213. Gregoire, C., et al., The trafficking of natural killer cells. Immunol Rev, 2007. 220: p. 169-82.

214. Walzer, T., et al., Natural killer cell trafficking in vivo requires a dedicated sphingosine 1-phosphate receptor. Nat Immunol, 2007. 8(12): p. 1337-44.

215. Wald, O., et al., IFN-gamma acts on T cells to induce NK cell mobilization and accumulation in target organs. J Immunol, 2006. 176(8): p. 4716-29.

216. Chen, S., et al., Suppression of tumor formation in lymph nodes by L-selectinmediated natural killer cell recruitment. J Exp Med, 2005. 202(12): p. 1679-89.

217. Martin-Fontecha, A., et al., Induced recruitment of NK cells to lymph nodes provides IFN-gamma for T(H)1 priming. Nat Immunol, 2004. 5(12): p. 1260-5.

218. Vivier, E., et al., Functions of natural killer cells. Nat Immunol, 2008. 9(5): p. 50310.

219. Smyth, M.J., et al., New aspects of natural-killer-cell surveillance and therapy of cancer. Nat Rev Cancer, 2002. 2(11): p. 850-61. 
220. Tomasello, E., et al., Signaling pathways engaged by NK cell receptors: double concerto for activating receptors, inhibitory receptors and NK cells. Semin Immunol, 2000. 12(2): p. 139-47.

221. Pegram, H.J., et al., Activating and inhibitory receptors of natural killer cells. Immunol Cell Biol, 2011. 89(2): p. 216-24.

222. Sivori, S., et al., TLR/NCR/KIR: Which One to Use and When? Front Immunol, 2014. 5: p. 105.

223. Farag, S.S., et al., Natural killer cell receptors: new biology and insights into the graft-versus-leukemia effect. Blood, 2002. 100(6): p. 1935-47.

224. Heidenreich, S., et al., Impact of the NK cell receptor LIR-1 (ILT-2/CD85j/LILRB1) on cytotoxicity against multiple myeloma. Clin Dev Immunol, 2012. 2012: p. 652130.

225. Chapman, T.L., A.P. Heikeman, and P.J. Bjorkman, The inhibitory receptor LIR-1 uses a common binding interaction to recognize class I MHC molecules and the viral homolog UL18. Immunity, 1999. 11(5): p. 603-13.

226. Saverino, D., et al., The CD85/LIR-1/ILT2 inhibitory receptor is expressed by all human $T$ lymphocytes and down-regulates their functions. J Immunol, 2000. 165(7): p. 3742-55.

227. Colonna, M., et al., A novel family of Ig-like receptors for HLA class I molecules that modulate function of lymphoid and myeloid cells. J Leukoc Biol, 1999. 66(3): p. 375-81.

228. Warren, H.S. and M.J. Smyth, NK cells and apoptosis. Immunol Cell Biol, 1999. 77(1): p. 64-75.

229. Lanier, L.L., et al., Structural and serological heterogeneity of gamma/delta T cell antigen receptor expression in thymus and peripheral blood. Eur J Immunol, 1988. 18(12): p. 1985-92.

230. Bryceson, Y.T., et al., Activation, coactivation, and costimulation of resting human natural killer cells. Immunol Rev, 2006. 214: p. 73-91.

231. Dardalhon, V., et al., CD226 is specifically expressed on the surface of Th1 cells and regulates their expansion and effector functions. J Immunol, 2005. 175(3): p. 1558-65.

232. Seth, S., et al., Heterogeneous expression of the adhesion receptor CD226 on murine NK and T cells and its function in NK-mediated killing of immature dendritic cells. J Leukoc Biol, 2009. 86(1): p. 91-101.

233. Chan, C.J., et al., DNAM-1/CD155 interactions promote cytokine and NK cellmediated suppression of poorly immunogenic melanoma metastases. J Immunol, 2010. 184(2): p. 902-11.

234. Shibuya, K., et al., Physical and functional association of LFA-1 with DNAM-1 adhesion molecule. Immunity, 1999. 11(5): p. 615-23.

235. Bottino, C., et al., Identification of PVR (CD155) and Nectin-2 (CD112) as cell surface ligands for the human DNAM-1 (CD226) activating molecule. J Exp Med, 2003. 198(4): p. 557-67.

236. Martinet, L., et al., DNAM-1 expression marks an alternative program of NK cell maturation. Cell Rep, 2015. 11(1): p. 85-97.

237. Zhang, Z., et al., DNAM-1 controls NK cell activation via an ITT-like motif. J Exp Med, 2015. 212(12): p. 2165-82.

238. Verhoeven, D.H., et al., NK cells recognize and lyse Ewing sarcoma cells through NKG2D and DNAM-1 receptor dependent pathways. Mol Immunol, 2008. 45(15): p. 3917-25. 
239. Lakshmikanth, T., et al., NCRs and DNAM-1 mediate NK cell recognition and lysis of human and mouse melanoma cell lines in vitro and in vivo. J Clin Invest, 2009. 119(5): p. 1251-63.

240. Cho, D., et al., Cytotoxicity of activated natural killer cells against pediatric solid tumors. Clin Cancer Res, 2010. 16(15): p. 3901-9.

241. Magri, G., et al., NKp46 and DNAM-1 NK-cell receptors drive the response to human cytomegalovirus-infected myeloid dendritic cells overcoming viral immune evasion strategies. Blood, 2011. 117(3): p. 848-56.

242. Matusali, G., et al., The human immunodeficiency virus type 1 Nef and Vpu proteins downregulate the natural killer cell-activating ligand PVR. J Virol, 2012. 86(8): p. 4496-504.

243. Hudspeth, K., B. Silva-Santos, and D. Mavilio, Natural cytotoxicity receptors: broader expression patterns and functions in innate and adaptive immune cells. Front Immunol, 2013. 4: p. 69.

244. Vitale, M., et al., NKp44, a novel triggering surface molecule specifically expressed by activated natural killer cells, is involved in non-major histocompatibility complex-restricted tumor cell lysis. J Exp Med, 1998. 187(12): p. 2065-72.

245. Fuchs, A., et al., Paradoxic inhibition of human natural interferon-producing cells by the activating receptor NKp44. Blood, 2005. 106(6): p. 2076-82.

246. Meresse, B., et al., Reprogramming of CTLs into natural killer-like cells in celiac disease. J Exp Med, 2006. 203(5): p. 1343-55.

247. Satoh-Takayama, N., et al., Microbial flora drives interleukin 22 production in intestinal NKp46+ cells that provide innate mucosal immune defense. Immunity, 2008. 29(6): p. 958-70.

248. Vivier, E., H. Spits, and T. Cupedo, Interleukin-22-producing innate immune cells: new players in mucosal immunity and tissue repair? Nat Rev Immunol, 2009. 9(4): p. 229-34.

249. Luci, C., et al., Influence of the transcription factor RORgammat on the development of NKp46+ cell populations in gut and skin. Nat Immunol, 2009. 10(1): p. 75-82.

250. Sanos, S.L., et al., RORgammat and commensal microflora are required for the differentiation of mucosal interleukin 22-producing NKp46+ cells. Nat Immunol, 2009. 10(1): p. 83-91.

251. Sternberg-Simon, M., et al., Natural killer cell inhibitory receptor expression in humans and mice: a closer look. Front Immunol, 2013. 4: p. 65.

252. Dimasi, N. and R. Biassoni, Structural and functional aspects of the Ly49 natural killer cell receptors. Immunol Cell Biol, 2005. 83(1): p. 1-8.

253. Ljutic, B., et al., Functional requirements for signaling through the stimulatory and inhibitory mouse NKR-P1 (CD161) NK cell receptors. J Immunol, 2005. 174(8): p. 4789-96.

254. Aust, J.G., et al., The expression and function of the NKRP1 receptor family in C57BL/6 mice. J Immunol, 2009. 183(1): p. 106-16.

255. Pozo, D., et al., CD161 (human NKR-P1A) signaling in NK cells involves the activation of acid sphingomyelinase. J Immunol, 2006. 176(4): p. 2397-406.

256. Lopez-Botet, M. and T. Bellon, Natural killer cell activation and inhibition by receptors for MHC class I. Curr Opin Immunol, 1999. 11(3): p. 301-7.

257. Borrego, F., et al., Structure and function of major histocompatibility complex (MHC) class I specific receptors expressed on human natural killer (NK) cells. Mol Immunol, 2002. 38(9): p. 637-60. 
258. Kaiser, B.K., et al., Interactions between NKG2x immunoreceptors and HLA-E ligands display overlapping affinities and thermodynamics. J Immunol, 2005. 174(5): p. 2878-84.

259. Vales-Gomez, M., et al., Kinetics and peptide dependency of the binding of the inhibitory NK receptor CD94/NKG2-A and the activating receptor CD94/NKG2-C to HLA-E. Embo j, 1999. 18(15): p. 4250-60.

260. Orr, M.T., et al., Development and function of CD94-deficient natural killer cells. PLoS One, 2010. 5(12): p. e15184.

261. Raulet, D.H., Roles of the NKG2D immunoreceptor and its ligands. Nat Rev Immunol, 2003. 3(10): p. 781-90.

262. Bauer, S., et al., Activation of NK cells and T cells by NKG2D, a receptor for stressinducible MICA. Science, 1999. 285(5428): p. 727-9.

263. Vivier, E., E. Tomasello, and P. Paul, Lymphocyte activation via NKG2D: towards a new paradigm in immune recognition? Curr Opin Immunol, 2002. 14(3): p. 30611.

264. Zhang, J., F. Basher, and J.D. Wu, NKG2D Ligands in Tumor Immunity: Two Sides of a Coin. Front Immunol, 2015. 6: p. 97.

265. Zwirner, N.W., et al., Cytokine-driven regulation of NK cell functions in tumor immunity: role of the MICA-NKG2D system. Cytokine Growth Factor Rev, 2007. 18(1-2): p. 159-70.

266. Muntasell, A., et al., Inhibition of NKG2D expression in NK cells by cytokines secreted in response to human cytomegalovirus infection. Blood, 2010. 115(25): p. 5170-9.

267. Guo, Q. and C. Zhang, Critical role of Toll-like receptor signaling in NK cell activation. Chinese Science Bulletin, 2012. 57(24): p. 3192-3202.

268. Sivori, S., et al., CPG and double-stranded RNA trigger human NK cells by Toll-like receptors: induction of cytokine release and cytotoxicity against tumors and dendritic cells. Proc Natl Acad Sci U S A, 2004. 101(27): p. 10116-21.

269. Guillerey, C., et al., Toll-like receptor 3 regulates NK cell responses to cytokines and controls experimental metastasis. Oncoimmunology, 2015. 4(9): p. e1027468.

270. Akazawa, T., et al., Antitumor NK activation induced by the Toll-like receptor 3TICAM-1 (TRIF) pathway in myeloid dendritic cells. Proc Natl Acad Sci U S A, 2007. 104(1): p. 252-7.

271. Pisegna, S., et al., p38 MAPK activation controls the TLR3-mediated up-regulation of cytotoxicity and cytokine production in human NK cells. Blood, 2004. 104(13): p. 4157-64.

272. Kawai, T. and S. Akira, Toll-like receptor and RIG-I-like receptor signaling. Ann N Y Acad Sci, 2008. 1143: p. 1-20.

273. McCartney, S., et al., Distinct and complementary functions of MDA5 and TLR3 in poly(I:C)-mediated activation of mouse NK cells. J Exp Med, 2009. 206(13): p. 2967-76.

274. Ngoi, S.M., M.G. Tovey, and A.T. Vella, Targeting poly(I:C) to the TLR3-independent pathway boosts effector CD8 T cell differentiation through IFN-alpha/beta. J Immunol, 2008. 181(11): p. 7670-80.

275. Maghazachi, A.A., G protein-coupled receptors in natural killer cells. J Leukoc Biol, 2003. 74(1): p. 16-24.

276. Taub, D.D., et al., Alpha and beta chemokines induce NK cell migration and enhance NK-mediated cytolysis. J Immunol, 1995. 155(8): p. 3877-88. 
277. al-Aoukaty, A., T.J. Schall, and A.A. Maghazachi, Differential coupling of CC chemokine receptors to multiple heterotrimeric G proteins in human interleukin-2activated natural killer cells. Blood, 1996. 87(10): p. 4255-60.

278. Robertson, M.J., Role of chemokines in the biology of natural killer cells. J Leukoc Biol, 2002. 71(2): p. 173-83.

279. Nieto, M., et al., Roles of chemokines and receptor polarization in NK-target cell interactions. J Immunol, 1998. 161(7): p. 3330-9.

280. Yoneda, O., et al., Fractalkine-mediated endothelial cell injury by NK cells. J Immunol, 2000. 164(8): p. 4055-62.

281. Rajasekaran, K., et al., Signaling by Fyn-ADAP via the Carma1-Bcl-10-MAP3K7 signalosome exclusively regulates inflammatory cytokine production in NK cells. Nat Immunol, 2013. 14(11): p. 1127-36.

282. Vivier, E., S. Ugolini, and J.A. Nunes, ADAPted secretion of cytokines in NK cells. Nat Immunol, 2013. 14(11): p. 1108-10.

283. Trapani, J.A., et al., Proapoptotic functions of cytotoxic lymphocyte granule constituents in vitro and in vivo. Curr Opin Immunol, 2000. 12(3): p. 323-9.

284. Dustin, M.L. and E.O. Long, Cytotoxic immunological synapses. Immunol Rev, 2010. 235(1): p. 24-34.

285. Podack, E.R., H. Hengartner, and M.G. Lichtenheld, A central role of perforin in cytolysis? Annu Rev Immunol, 1991. 9: p. 129-57.

286. Orange, J.S., Formation and function of the lytic NK-cell immunological synapse. Nat Rev Immunol, 2008. 8(9): p. 713-25.

287. Cohnen, A., et al., Surface CD107a/LAMP-1 protects natural killer cells from degranulation-associated damage. Blood, 2013. 122(8): p. 1411-8.

288. Cooper, M.A., T.A. Fehniger, and M.A. Caligiuri, The biology of human natural killer-cell subsets. Trends Immunol, 2001. 22(11): p. 633-40.

289. Kagi, D., et al., Cytotoxicity mediated by $T$ cells and natural killer cells is greatly impaired in perforin-deficient mice. Nature, 1994. 369(6475): p. 31-7.

290. Smyth, M.J., et al., Perforin is a major contributor to NK cell control of tumor metastasis. J Immunol, 1999. 162(11): p. 6658-62.

291. Stepp, S.E., et al., Perforin gene defects in familial hemophagocytic lymphohistiocytosis. Science, 1999. 286(5446): p. 1957-9.

292. Smyth, M.J., et al., Cytokines in cancer immunity and immunotherapy. Immunol Rev, 2004. 202: p. 275-93.

293. Motyka, B., et al., Mannose 6-phosphate/insulin-like growth factor II receptor is a death receptor for granzyme $B$ during cytotoxic $T$ cell-induced apoptosis. Cell, 2000. 103(3): p. 491-500.

294. Alter, G., J.M. Malenfant, and M. Altfeld, CD107a as a functional marker for the identification of natural killer cell activity. J Immunol Methods, 2004. 294(1-2): p. 15-22.

295. Bryceson, Y.T., et al., Cytolytic granule polarization and degranulation controlled by different receptors in resting NK cells. J Exp Med, 2005. 202(7): p. 1001-12.

296. Zamai, L., et al., Natural killer (NK) cell-mediated cytotoxicity: differential use of TRAIL and Fas ligand by immature and mature primary human NK cells. J Exp Med, 1998. 188(12): p. 2375-80.

297. Chester, C., K. Fritsch, and H.E. Kohrt, Natural Killer Cell Immunomodulation: Targeting Activating, Inhibitory, and Co-stimulatory Receptor Signaling for Cancer Immunotherapy. Front Immunol, 2015. 6: p. 601. 
298. Cooper, M.A., et al., Human natural killer cells: a unique innate immunoregulatory role for the CD56(bright) subset. Blood, 2001. 97(10): p. 3146-51.

299. Carson, W.E., et al., Interleukin (IL) 15 is a novel cytokine that activates human natural killer cells via components of the IL-2 receptor. J Exp Med, 1994. 180(4): p. 1395-403.

300. Grimm, E.A., et al., Lymphokine-activated killer cell phenomenon. Lysis of natural killer-resistant fresh solid tumor cells by interleukin 2-activated autologous human peripheral blood lymphocytes. J Exp Med, 1982. 155(6): p. 1823-41.

301. Cooper, M.A., et al., Cytokine-induced memory-like natural killer cells. Proc Natl Acad Sci U S A, 2009. 106(6): p. 1915-9.

302. Nguyen, K.B., et al., Coordinated and distinct roles for IFN-alpha beta, IL-12, and IL15 regulation of $N K$ cell responses to viral infection. J Immunol, 2002. 169(8): p. 4279-87.

303. Zucchini, N., et al., Natural killer cells in immunodefense against infective agents. Expert Rev Anti Infect Ther, 2008. 6(6): p. 867-85.

304. Loza, M.J., et al., Expression of type 1 (interferon gamma) and type 2 (interleukin13, interleukin-5) cytokines at distinct stages of natural killer cell differentiation from progenitor cells. Blood, 2002. 99(4): p. 1273-81.

305. Stetson, D.B., et al., Constitutive cytokine mRNAs mark natural killer (NK) and NKT cells poised for rapid effector function. J Exp Med, 2003. 198(7): p. 1069-76.

306. Boehm, U., et al., Cellular responses to interferon-gamma. Annu Rev Immunol, 1997. 15: p. 749-95.

307. Schoenborn, J.R., et al., Comprehensive epigenetic profiling identifies multiple distal regulatory elements directing transcription of the gene encoding interferongamma. Nat Immunol, 2007. 8(7): p. 732-42.

308. Li, M.O., et al., Transforming growth factor-beta regulation of immune responses. Annu Rev Immunol, 2006. 24: p. 99-146.

309. Schindler, $\mathrm{H}$., et al., The production of IFN-gamma by IL-12/IL-18-activated macrophages requires STAT4 signaling and is inhibited by IL-4. J Immunol, 2001. 166(5): p. 3075-82.

310. Dhiman, R., et al., IL-22 produced by human NK cells inhibits growth of Mycobacterium tuberculosis by enhancing phagolysosomal fusion. J Immunol, 2009. 183(10): p. 6639-45.

311. Horowitz, A., K.A. Stegmann, and E.M. Riley, Activation of natural killer cells during microbial infections. Front Immunol, 2011. 2: p. 88.

312. Topham, N.J. and E.W. Hewitt, Natural killer cell cytotoxicity: how do they pull the trigger? Immunology, 2009. 128(1): p. 7-15.

313. Smyth, M.J., et al., Activation of NK cell cytotoxicity. Mol Immunol, 2005. 42(4): p. 501-10.

314. Jaeger, B.N. and E. Vivier, Natural killer cell tolerance: control by self or selfcontrol? Cold Spring Harb Perspect Biol, 2012. 4(3).

315. Karre, K., Natural killer cell recognition of missing self. Nat Immunol, 2008. 9(5): p. 477-80.

316. Uhrberg, M., et al., Human diversity in killer cell inhibitory receptor genes. Immunity, 1997. 7(6): p. 753-63.

317. Raulet, D.H. and R.E. Vance, Self-tolerance of natural killer cells. Nat Rev Immunol, 2006. 6(7): p. 520-31.

318. Hoglund, P. and P. Brodin, Current perspectives of natural killer cell education by MHC class I molecules. Nat Rev Immunol, 2010. 10(10): p. 724-34. 
319. Gasser, S. and D.H. Raulet, Activation and self-tolerance of natural killer cells. Immunol Rev, 2006. 214: p. 130-42.

320. Brodin, P., et al., The strength of inhibitory input during education quantitatively tunes the functional responsiveness of individual natural killer cells. Blood, 2009. 113(11): p. 2434-41.

321. Joncker, N.T., et al., NK cell responsiveness is tuned commensurate with the number of inhibitory receptors for self-MHC class I: the rheostat model. J Immunol, 2009. 182(8): p. 4572-80.

322. Chalifour, A., et al., A Role for cis Interaction between the Inhibitory Ly49A receptor and MHC class I for natural killer cell education. Immunity, 2009. 30(3): p. 337-47.

323. Kerdiles, Y., S. Ugolini, and E. Vivier, T cell regulation of natural killer cells. J Exp Med, 2013. 210(6): p. 1065-8.

324. Narni-Mancinelli, E., S. Ugolini, and E. Vivier, Tuning the threshold of natural killer cell responses. Curr Opin Immunol, 2013. 25(1): p. 53-8.

325. Narni-Mancinelli, E., et al., Tuning of natural killer cell reactivity by NKp46 and Helios calibrates T cell responses. Science, 2012. 335(6066): p. 344-8.

326. Elliott, J.M., J.A. Wahle, and W.M. Yokoyama, MHC class I-deficient natural killer cells acquire a licensed phenotype after transfer into an MHC class I-sufficient environment. J Exp Med, 2010. 207(10): p. 2073-9.

327. Watzl, C., et al., Natural killer cell regulation - beyond the receptors. F1000Prime Rep, 2014. 6: p. 87.

328. Fehniger, T.A., et al., Acquisition of murine NK cell cytotoxicity requires the translation of a pre-existing pool of granzyme $B$ and perforin mRNAs. Immunity, 2007. 26(6): p. 798-811.

329. Walzer, T., et al., Natural-killer cells and dendritic cells: "l'union fait la force". Blood, 2005. 106(7): p. 2252-8.

330. Bryceson, Y.T. and E.O. Long, Line of attack: NK cell specificity and integration of signals. Curr Opin Immunol, 2008. 20(3): p. 344-52.

331. Lucas, M., et al., Dendritic cells prime natural killer cells by trans-presenting interleukin 15. Immunity, 2007. 26(4): p. 503-17.

332. Long, E.O., Ready for prime time: NK cell priming by dendritic cells. Immunity, 2007. 26(4): p. 385-7.

333. Bihl, F., et al., Primed antigen-specific CD4+ $T$ cells are required for $N K$ cell activation in vivo upon Leishmania major infection. J Immunol, 2010. 185(4): p. 2174-81.

334. Ghiringhelli, F., et al., CD4+CD25+ regulatory $T$ cells inhibit natural killer cell functions in a transforming growth factor-beta-dependent manner. J Exp Med, 2005. 202(8): p. 1075-85.

335. Smyth, M.J., et al., CD4+CD25+ T regulatory cells suppress NK cell-mediated immunotherapy of cancer. J Immunol, 2006. 176(3): p. 1582-7.

336. Kottke, T., et al., Use of biological therapy to enhance both virotherapy and adoptive T-cell therapy for cancer. Mol Ther, 2008. 16(12): p. 1910-8.

337. Pandiyan, $\mathrm{P}$., et al., $C D 4+C D 25+F o x p 3+$ regulatory $T$ cells induce cytokine deprivation-mediated apoptosis of effector CD4+ T cells. Nat Immunol, 2007. 8(12): p. 1353-62.

338. O'Sullivan, T.E., J.C. Sun, and L.L. Lanier, Natural Killer Cell Memory. Immunity, 2015. 43(4): p. 634-45.

339. Jamieson, A.M., et al., Turnover and proliferation of NK cells in steady state and lymphopenic conditions. J Immunol, 2004. 172(2): p. 864-70. 
340. O'Leary, J.G., et al., T cell- and B cell-independent adaptive immunity mediated by natural killer cells. Nat Immunol, 2006. 7(5): p. 507-16.

341. Paust, S., et al., Critical role for the chemokine receptor CXCR6 in NK cell-mediated antigen-specific memory of haptens and viruses. Nat Immunol, 2010. 11(12): p. 1127-35.

342. Sun, J.C., et al., NK cells and immune "memory". J Immunol, 2011. 186(4): p. 18917.

343. Arase, H., et al., Direct recognition of cytomegalovirus by activating and inhibitory NK cell receptors. Science, 2002. 296(5571): p. 1323-6.

344. Sun, J.C., J.N. Beilke, and L.L. Lanier, Adaptive immune features of natural killer cells. Nature, 2009. 457(7229): p. 557-61.

345. Koka, R., et al., Cutting edge: murine dendritic cells require IL-15R alpha to prime NK cells. J Immunol, 2004. 173(6): p. 3594-8.

346. Della Chiesa, M., et al., The natural killer cell-mediated killing of autologous dendritic cells is confined to a cell subset expressing CD94/NKG2A, but lacking inhibitory killer Ig-like receptors. Eur J Immunol, 2003. 33(6): p. 1657-66.

347. Degli-Esposti, M.A. and M.J. Smyth, Close encounters of different kinds: dendritic cells and NK cells take centre stage. Nat Rev Immunol, 2005. 5(2): p. 112-24.

348. Pallmer, K. and A. Oxenius, Recognition and Regulation of T Cells by NK Cells. Front Immunol, 2016. 7.

349. Deauvieau, F., et al., Lessons from NK Cell Deficiencies in the Mouse. Curr Top Microbiol Immunol, 2016. 395: p. 173-90.

350. Johansson, S., et al., NK cells: elusive players in autoimmunity. Trends Immunol, 2005. 26(11): p. 613-8.

351. Orange, J.S. and Z.K. Ballas, Natural killer cells in human health and disease. Clin Immunol, 2006. 118(1): p. 1-10.

352. Mandelboim, 0., et al., Recognition of haemagglutinins on virus-infected cells by NKp46 activates lysis by human NK cells. Nature, 2001. 409(6823): p. 1055-60.

353. Scalzo, A.A., et al., The interplay between host and viral factors in shaping the outcome of cytomegalovirus infection. Immunol Cell Biol, 2007. 85(1): p. 46-54.

354. Tobin, A.M., et al., Natural killer cells in psoriasis. J Innate Immun, 2011. 3(4): p. 403-10.

355. Zhang, B., et al., Regulation of experimental autoimmune encephalomyelitis by natural killer (NK) cells. J Exp Med, 1997. 186(10): p. 1677-87.

356. Xu, W., et al., Mechanism of natural killer (NK) cell regulatory role in experimental autoimmune encephalomyelitis. J Neuroimmunol, 2005. 163(1-2): p. 24-30.

357. Schuster, I.S., et al., TRAIL + NK cells control CD4+ T cell responses during chronic viral infection to limit autoimmunity. Immunity, 2014. 41(4): p. 646-56.

358. Enk, J. and O. Mandelboim, The Role of Natural Cytotoxicity Receptors in Various Pathologies: Emphasis on Type I Diabetes. Front Immunol, 2014. 5.

359. Gur, C., et al., Recognition and killing of human and murine pancreatic beta cells by the NK receptor NKp46. J Immunol, 2011. 187(6): p. 3096-103.

360. Dalbeth, N. and M.F. Callan, A subset of natural killer cells is greatly expanded within inflamed joints. Arthritis Rheum, 2002. 46(7): p. 1763-72.

361. Mandal, A. and C. Viswanathan, Natural killer cells: In health and disease. Hematol Oncol Stem Cell Ther, 2015. 8(2): p. 47-55.

362. Ong, S., N.R. Rose, and D. Cihakova, Natural killer cells in inflammatory heart disease. Clin Immunol, 2017. 175: p. 26-33. 
363. Erkeller-Yuksel, F.M., P.M. Lydyard, and D.A. Isenberg, Lack of NK cells in lupus patients with renal involvement. Lupus, 1997. 6(9): p. 708-12.

364. Jonasson, L., K. Backteman, and J. Ernerudh, Loss of natural killer cell activity in patients with coronary artery disease. Atherosclerosis, 2005. 183(2): p. 316-21.

365. Backteman, K., J. Ernerudh, and L. Jonasson, Natural killer (NK) cell deficit in coronary artery disease: no aberrations in phenotype but sustained reduction of NK cells is associated with low-grade inflammation. Clin Exp Immunol, 2014. 175(1): p. 104-12.

366. Ayach, B.B., et al., Stem cell factor receptor induces progenitor and natural killer cell-mediated cardiac survival and repair after myocardial infarction. Proc Natl Acad Sci U S A, 2006. 103(7): p. 2304-9.

367. Colucci, F., M.A. Caligiuri, and J.P. Di Santo, What does it take to make a natural killer? Nat Rev Immunol, 2003. 3(5): p. 413-25.

368. Bouchentouf, M., et al., Induction of cardiac angiogenesis requires killer cell lectinlike receptor 1 and alpha4beta 7 integrin expression by NK cells. J Immunol, 2010. 185(11): p. 7014-25.

369. Godeny, E.K. and C.J. Gauntt, Involvement of natural killer cells in coxsackievirus B3-induced murine myocarditis. J Immunol, 1986. 137(5): p. 1695-702.

370. Young, L.H., et al., Perforin-mediated myocardial damage in acute myocarditis. Lancet, 1990. 336(8722): p. 1019-21.

371. Kosierkiewicz, T.A., S.M. Factor, and D.W. Dickson, Immunocytochemical studies of atherosclerotic lesions of cerebral berry aneurysms. J Neuropathol Exp Neurol, 1994. 53(4): p. 399-406.

372. Whitman, S.C., et al., Depletion of natural killer cell function decreases atherosclerosis in low-density lipoprotein receptor null mice. Arterioscler Thromb Vasc Biol, 2004. 24(6): p. 1049-54.

373. Bobryshev, Y.V. and R.S. Lord, Identification of natural killer cells in human atherosclerotic plaque. Atherosclerosis, 2005. 180(2): p. 423-7.

374. Selathurai, A., et al., Natural killer (NK) cells augment atherosclerosis by cytotoxicdependent mechanisms. Cardiovasc Res, 2014. 102(1): p. 128-37.

375. Clerc, G. and P.M. Rouz, Lymphocyte subsets in severe atherosclerosis before revascularization. Ann Intern Med, 1997. 126(12): p. 1004-5.

376. Bruunsgaard, H., et al., Decreased natural killer cell activity is associated with atherosclerosis in elderly humans. Exp Gerontol, 2001. 37(1): p. 127-36.

377. Ward, D.M., et al., Use of expression constructs to dissect the functional domains of the CHS/beige protein: identification of multiple phenotypes. Traffic, 2003. 4(6): p. 403-15.

378. Paigen, B., et al., Atherosclerosis susceptibility differences among progenitors of recombinant inbred strains of mice. Arteriosclerosis, 1990. 10(2): p. 316-23.

379. Schiller, N.K., W.A. Boisvert, and L.K. Curtiss, Inflammation in atherosclerosis: lesion formation in LDL receptor-deficient mice with perforin and Lyst(beige) mutations. Arterioscler Thromb Vasc Biol, 2002. 22(8): p. 1341-6.

380. Roder, J.C., et al., A new immunodeficiency disorder in humans involving NK cells. Nature, 1980. 284(5756): p. 553-5.

381. Linton, M.F., A.S. Major, and S. Fazio, Proatherogenic role for NK cells revealed. Arterioscler Thromb Vasc Biol, 2004. 24(6): p. 992-4.

382. To, K., et al., NKT cell subsets mediate differential proatherogenic effects in ApoE-/mice. Arterioscler Thromb Vasc Biol, 2009. 29(5): p. 671-7. 
383. Oberg, L., et al., Expression of Ly49A on $T$ cells alters the threshold for $T$ cell responses. Eur J Immunol, 2000. 30(10): p. 2849-56.

384. Tupin, E., et al., CD1d-dependent activation of NKT cells aggravates atherosclerosis. J Exp Med, 2004. 199(3): p. 417-22.

385. Nishikado, H., et al., NK cell-depleting anti-asialo GM1 antibody exhibits a lethal off-target effect on basophils in vivo. J Immunol, 2011. 186(10): p. 5766-71.

386. Wiltrout, R.H., et al., Reactivity of anti-asialo GM1 serum with tumoricidal and nontumoricidal mouse macrophages. J Leukoc Biol, 1985. 37(5): p. 597-614.

387. Trambley, J., et al., Asialo GM1(+) CD8(+) T cells play a critical role in costimulation blockade-resistant allograft rejection. J Clin Invest, 1999. 104(12): p. 1715-22.

388. Lee, U., et al., Murine asialo GM1+CD8+ T cells as novel interleukin-12-responsive killer T cell precursors. Jpn J Cancer Res, 1996. 87(5): p. 429-32.

389. Murray, C.J., et al., Disability-adjusted life years (DALYs) for 291 diseases and injuries in 21 regions, 1990-2010: a systematic analysis for the Global Burden of Disease Study 2010. Lancet, 2012. 380(9859): p. 2197-223.

390. Libby, P., A.H. Lichtman, and G.K. Hansson, Immune Effector Mechanisms Implicated in Atherosclerosis: From Mice to Humans. Immunity, 2013. 38(6): p. 1092-1104.

391. Kyaw, T., et al., Cytotoxic and proinflammatory CD8+ T lymphocytes promote development of vulnerable atherosclerotic plaques in apoE-deficient mice. Circulation, 2013. 127(9): p. 1028-39.

392. Xia, M., et al., Immune activation resulting from NKG2D/ligand interaction promotes atherosclerosis. Circulation, 2011. 124(25): p. 2933-43.

393. Berahovich, R.D., et al., Evidence for NK Cell Subsets Based on Chemokine Receptor Expression. The Journal of Immunology, 2006. 177(11): p. 7833-7840.

394. Umehara, H., et al., Fractalkine in vascular biology: from basic research to clinical disease. Arterioscler Thromb Vasc Biol, 2004. 24(1): p. 34-40. 


\title{
Résumé
}

L'inflammation chronique en réponse à l'accumulation de lipoprotéines dans la paroi artérielle est centrale dans le développement de l'athérosclérose. L'immunité innée et adaptée sont impliquées dans ce processus. Les cellules Natural Killer (NK), un des éléments clés de l'immunité innée, ont été identifiées dans les lésions athérosclérotiques humaines et murines. Bien que plusieurs études aient cherché à évaluer le rôle des cellules NK dans des modèles animaux expérimentaux d'athérosclérose,, les résultats restent contradictoires, certaines rapportant des effets pro-athérogéniques, d'autres anti-athérogéniques.

L'une des principales limites de ces études est le manque de spécificité dans le ciblage de la perte ou du gain de fonction des cellules NK. Nous avons utilisé deux approches génétiques sélectives pour étudier le rôle des cellules NK dans l'athérosclérose: 1) des souris $\mathrm{Ncr}^{i \mathrm{Cr} /+} R 26^{I s I D T A /+}$ dans lesquelles les cellules NK ont été déplétées 2) des souris Noé, dont les cellules NK sont hyper-réactives. Les cellules de la moelle osseuse (BM) de ces souris ont été utilisées pour reconstituer le système hématopoiétique de souris $L d l r \%$ irradiées. Après une période de récupération de 4 semaines, les souris ont été mises sous un régime riche en matières grasses (HFD) pendant 8 semaines. L'analyse morphométrique de la taille des lésions 'athérosclérose dans le sinus aortique et l'aorte thoracique n'a montré aucune différence statistiquement significative entre les 3 groupes. De plus, aucune différence n'a été observée dans la composition de la plaque en termes de teneur en collagène, d'infiltration de macrophages ou de profil immunitaire dans le sang et la rate des souris $\mathrm{Ncr}^{i C r e} R 26^{\text {Isl-DTA }}$, Noé ou contrôles. Nous avons ensuite étudié la sélectivité de des anticorps anti-asialo-GM1 dans la déplétion des cellules NK, qui avaient été utilisés précédemment pour démontrer le rôle proathérogène des cellules NK. Nous avons confirmé les effets non spécifiques de cet anticorps, qui déplète non seulement les cellules NK, mais aussi les lymphocytes NKT et CD8+. Enfin, pour déterminer si l'activation des cellules NK par un stimulus externe pouvait avoir des effets sur l'athérosclérose, nous avons traité les souris chimériques (souris $L d l r \%$ irradiées reconstituées soit avec les cellules de moelle contrôle ou déficiente en cellules NK) avec du poly IC (un mimétique viral) pendant 8 semaines de HFD. Nous avons trouvé une réduction significative de la taille des lésions au niveau du sinus aortique et de l'aorte thoracique dans les souris déficientes en cellules NK.

Nos résultats, à partir de modèles de souris spécifiques, contredisent les études antérieures et démontrent clairement que chez les souris hypercholestérolémiques, les cellules NK n'ont aucun effet direct sur l'athérosclérose, sauf si elles sont pré-stimulée, comme par exemple dans un contexte d'infection virale ou de présence de tumeurs.

\begin{abstract}
Chronic inflammation is central in the development of atherosclerosis. Both innate and adaptive immunity are involved in this process. Although several studies have evaluated the functions of NK cells in experimental animal models of atherosclerosis, it is not yet clear whether NK cells behave as protective or pro-atherogenic effectors. One of the main caveats of previous studies was the lack of specificity in targeting loss- or gain-of-function of NK cells. Here, we used two selective genetic approaches to investigate the role of NK cells in atherosclerosis: 1) $\mathrm{Ncrl}^{\mathrm{iCre} /+} R 26^{\mathrm{IsIDTA} /+}$ mice in which NK cells were depleted, 2) Noé mice in which NK cells are hyperresponsive. No difference in atherosclerotic lesion size was found in $L d l r^{--}$mice transplanted with bone marrow cells from $N c r I^{i C r e} R 26 R^{l s l-D T A}$, Noé or WT mice. Also, no difference was observed in plaque composition in terms of collagen content, macrophage infiltration or the immune profile in blood and spleen, although Noé chimera had more IFN- $\square$-producing NK cells in comparison with WT mice. Then, we investigated the NK cell selectivity of anti-asialo GM1 anti-serum, which was previously used to conclude to the pro-atherogenicity of NK cells. Anti-asialo GM1 treatment decreased atherosclerosis in both $L d l r^{--}$mice transplanted with $\mathrm{Ncrl}^{i \mathrm{Cre}} \mathrm{R} 26 \mathrm{R}^{\mathrm{sl}-D T A}$ or WT BM, indicating that its anti-atherogenic effects are unrelated to NK cell depletion. Finally, to determine whether NK cells could contribute to the disease in conditions of pathological NK cell overactivation, we treated irradiated $L d l r^{--}$mice reconstituted with either WT or $N c r I^{i C r e} R 26 R^{I s l-D T A}$ BM with the viral mimic Poly(I:C) and found a significant reduction of plaque size in NK-cell deficient chimeric mice.

Our findings, using state-of-the-art mouse models, clearly demonstrate that NK cells have no direct effect on the natural development of hypercholesterolemia-induced atherosclerosis, but may play a role when an additional systemic NK cell overactivation occurs.
\end{abstract}

Key words: Atherosclerosis, Immune System, Inflammation, Natural Killer Cells, 